\title{
THE GROUPING OF AFFERENT IMPULSES WITHIN THE SPINAL CORD.
}

\author{
BY HENRT HEAD, M.D., F.R.S. \\ Physician to the London Hospital. \\ YD \\ THEODORE THOMPSON, M.D. \\ Asistant Physician to the Lundon Hospital, and to the Hospital for \\ Sick Childron.
}

OHapter I. - Introduction.

Methods.

OHaptea II. - The Nature of the Loss Seasation produced by a Lesion of the Spinal Cord, compared with that due to Division of Peripheral Nervos.

Section 1.-Paiv.

Section 2.- Heat and Cold.

Section 3.- Light Touch and Doop Touch

Section 4. - Pessive Position and Movement.

Section 5.-The Compass.Test.

Craptar III.-The Intor-Relation of Afferent Impulses in their Passage up the Spinal Cord.

Section 1.-Pain, Heat and Cold.

Saction 3.--Touch and Pressure.

Section 3.-Sense of Passive Position.

Section 4. -The Relation between the Power of Discriminating Two Points and the other Forms of Bensibility.

Chaptiz IV.-Locel Effects of an Intramedullary Lesion.

Saction 1. The Local Sensory Effects of an Intramednllary Lesion ocour on the same Bide as the Disturbance of Motion.

Section 2. - The Nature of the Local Disturbance of Sensation produced by an Intramedullary Lesion.

Chaptra V.-Afferent Segmentetion within the Spinel Cord.

Ohaptrer VI. - The Pasage through the Bpinal Cord of Afferent Impalses Conoerned with "Lrocalisation."

Section 1. -The Impulses Concerned with the Reoognition of Passive Position and Movement.

Section 2.-The Impulses Concerned with Tactile Discrimination.

Section 8. - Tactile Irocalisation.

Section 4.-Summary.

OHapter VII - General Conclusions.

APPendI_-Reports of the Cases Cited in this Paper.

VOL. XXIX. 


\section{Chapter I.}

\section{Introduction.}

IT would seem reasonable to suppose that the mechanism of sensation was arranged in such a way that, from the skin to the sensorium, there was one system of end-organs, fibres and cells for each sensory quality appreciable by man. On this supposition, every application of heat to the skin would cause a disturbance in the activity of a special set of endorgans, which reacted to this form of stimulation only. From these end-organs the impulse would be transferred by specialised fibres to the spinal cord, and thence by a single tract, directly or through the intermediation of cell-systems, to the final centres in the brain. On this hypothesis, similar organs must exist for cold, for pain, for pressure and for touch. With the discovery of the hot and cold spots, and with von Frey's [10] further development of the doctrine of punctate sensibility, a mechanism seemed to have been found which would reasonably explain the phenomena of sensation.

But recent work has shown'that, as far as the peripheral nerves are concerned, the afferent nervous system is not arranged on so simple a plan. Rivers [23], in conjunction with one of us, has been able to show that between the sparsely scattered hot spots lies some otber mechanism, capable of responding to stimulation by warm objects. Similarly, the work of Sherren [13] upon divided peripheral nerves has demonstrated the existence of two afferent systems in the skin, each of which has its own distribution. To explain the natare and distribution of the sensory mechanism in the peripheral nerves, we are compelled to fall back upon a hypothesis of evolution, from a simple apparatus to something higher but still imperfect; we cannot assume the immediate development of a system logically and specifically complete.

The conception, which we bave reached, of the afferent mechanism in the peripheral parts of the nervous system, accords more clearly with what might be expected from our present knowledge of comparative anatomy and physio- 
logy. For it is extremely unlikely that, out of the primitive sense-organs of lower animals, independent systems could be developed in man, specifically fitted to receive all those impulses which lead to the development of the sensations of beat, of cold, of pain, of touch, or of pressure. But our doctrine of three peripheral systems developed in all probability at different phylogenetic periods, each system reacting differently to the mass-stimuli of daily life, accords better with the known processes of evolution.

We concluded from our examination of the phenomena which follow division of peripheral nerves, that the afferent mechanism at this level of the nervous system consisted of three systems :-

(1) A system corresponding to the group of impulses we have called deep sensibility. The end-organs of this system respond to the stimulas of pressure and to the movement of joints, tendons and muscles. Painful impulses can also arise within this system in consequence of injury to a joint, or excessive pressure. This sensory mechanism is capable of responding in such a way that the patient appreciates both the locality of the stimulus and the direction of movement in any joint which lies within an area innervated solely by this system; and yet the integrity of deep sensibility carries with it no power of appreciating a stimulus, such as that of cotton-wool, even over hairy parts. Nor does it permit of the discrimination of two compass-points applied simultaneously to the skin, even when widely separated.

The fibres which conduct these sensory impulses run mainly with the muscular nerves, and are not destroyed by division of all the sensory nerves to the skin.

(2) The protopathic system, capable of responding to painful cutaneous stimuli and to the more extreme degrees of heat and cold. Its end-organs are grouped in points on the surface of the body, sensitive to one only of these stimuli. Their response is diffuse, and unaccompanied by any definite appreciation of the locality of the spot stimulated.

(3) The epicritic system. To the impulses of this system we owe the power of cataneous localisation, of discriminating 
two points and of recognising the finer grades of temperature, called cool and warm.

A knowledge of this grouping of afferent impulses was gained by studying the consequences which followed division of peripheral nerves and posterior nerve roots in man. But, on comparing the loss of sensation produced by injury and disease of the spinal cord with that caused by division of peripheral nerves, fundamental differences at once became apparent. For if, in consequence of disease of the spinal cord, sensibility to the pain of a prick was lost, the pain caused by excessive pressure was also abolished, even though the parts remained otherwise normally sensitive to pressure. Similarly, when a part became totally insensitive to stimuli which usually evoke a sensation of warmth, it was found to be also insensitive to all temperatures which normally cause a sensation of heat.

It is evident, therefore, that, as soon as a sensory impulse reaches its first junction in the spinal cord, it becomes shunted into tracts devoted to the conduction of impulses, grouped in a way different from that found in the peripheral nerves. It is no longer a question of protopathic, epicritic and deep sensibility; esch tract in the central nervous system is devoted to the conduction of one of the specific impulses, such as pain, touch, heat and cold.'

In the present paper, we are concerned more directly with this grouping of sensory impulses in the spinal cord, rather than with the position of the tracts by which they pass to the higher centres. We hope to demonstrate, not only that a change takes place in the grouping of the afferent impulses, but also to show at what point this transformation occurs.

\section{Methods.}

The cases quoted in this paper occurred mainly in the practice of the London Hospital, but we have included some instances from the private practice of one of us. Most of the patients have been under observation for long periods, in some instances for over five years, and we have thus been able to gain an idea of their temperament and trustworthi-

' For the nature of the posterior columns vide Chap. VII., p. 686. 
ness. For it must be remembered that observations, extending in many iustances over large areas of the body, require the exercise of much greater patience and exactitude than the shorter examinations of the smaller areas of loss of sensation produced by injury to peripheral nerves. In many instances, we have rejected all our observations on a patient on account of his unsatisfactory mental state. Moreover, fatigue plays a considerable part in the answers obtained to such tests as we have applied, and we have therefore as far as possible made our observations in short but frequently-repeated sittings.

As far as possible, our observations were made in a quiet room, apart from the hospital ward with its distracting sights and sounds. When it was necessary, in consequence of the difficulty in transporting the patient, to carry out our tests in a ward, his bed was carefully screened and every method adopted to secure his undivided attention. In all cases the patient was in bed, and the parts to be tested were exposed as little as possible. In the winter, the accuracy with which replies were given over parts of diminished sensibility was, as a rule, less than in the summer. This is not altogether a matter of external temperature; for it was found that a damp, misty or foggy day was peculiarly uufavourable for testing sensation. Anything that produces a feeling of coldness, anything which causes shivering or the appearance of "goose skin," greatly diminishes the accuracy of the answers given to most tests. The most favourable conditions are a warm day of early summer, or a bright cool winter morning in a well-warmed room.

It is important that the patient should be free from all visceral discomfort; he must not be hungry, nor suffer from a desire to empty his bladder. During the testing of R. A. H. (Case 16) it was noticed that his answers became much less accurate than they had been earlier in the day; for, although the right forearm was sensitive to touch and at any rate not totally insensitive to a prick, he failed eight times out of twenty attempts to tell the head from the point of a pin. He was then allowed to empty his bladder, and from that time made no further mistakes. His answers, which had shown much confusion, were now uniformly correct 
During the testing, the patient's eyes were closed; or in some instances, especially where the area under observation lay over the lower extremities, he was allowed to lie with his eyes open, but a screen was placed across the bed so that he was unable to see the hands of the operator or the tests to be applied. Some patients are more comfortable when this system is adopted, than if they are forced to remain for long periods with closed eyes.

Superficial touch was tested by means of cotton-wool, stroked gently across the part. Unfortunately, this stimulus, so perfect a test over parts endowed with hair, is untrustworthy over hairless parts, unless used with extreme caution. Many brands of wool, when rolled into a wisp, form so stiff a mass that deep sensibility (pressure) is evoked; but if this cotton-wool is frayed out, so that it can evoke sensations of light touch only, it frequently fails to stimulate the horny paim of a workman, or even some parts of a normal, wellkept hand.

In some instances we have used a set of hairs, kindly prepared for one of as by Professor von Frey. Each consists of a hair of different resistance to bending, fixed to a small wooden rod at a right angle. These hairs are calibrated by determining upon a small balance the pressure necessary to bend them. We wish to protest against a common variation of von Frey's apparatus, which consists of a single hair in a metal sheath, so arranged that it can be protruded or withdrawn to a varying extent. The condition of such a hair varies greatly, and the pressure necessary to bend it varies from time to time, even when it is extruded from its sheath to the same amount. Moreover, in order that the hair may not suffer by the extension and withdrawal, it must of necessity be thicker and coarser than when the test is made with a series of hairs, each permanently affixed to its own bandle. ${ }^{1}$

1 The actual hairs used by as, which we owe to the kindness of Professor von Frey, bent at the following pressures: No. 8 at $890 \mathrm{mgm}$., No. 6 at $860 \mathrm{mgm}$., No. 4 at $230 \mathrm{mgm}$., No. 2 at $100 \mathrm{mgm}$. These hairs are kept in a box with the hendles supported on a rack so that the hair remains always free from contact. The force required to bend them varies slightly according to use and the condition of the atmosphere; but after nearly four years No. 8 still turns the scale at $800 \mathrm{mgm}$., No. 5 at $320 \mathrm{mgm}$., No. 4 at $210 \mathrm{mgm}$. 
The power of discrimivating two points was tested with a pair of compasses, the ends of which had been blunted. The method adopted differs from that in general use, and is an amplification of that suggested by McDougall.' The compass-points were set at a certain distance from one another; they were then applied to the part to be tested in such a way that sometimes two points, sometimes one point only touched the skin. No questions were asked, but, before the testing began, the patient, whose eyes were closed, was told to say with every application how many points he perceived. The stimuli followed one another in an entirely irregular order, but so that, ultimately, the patient had been touched ten times with one point, ten times with two points. Each series was recorded in such a manner, that it became inimediately obvious in how many cases among the ten single, and among the ten double stimuli, the patieut had given a wroug answer.

In some of these experiments it was impossible to compare the results obtained over an abnormal part with a similar part of normal sensibility. When we were examining the consequences of injury to a peripheral nerve of the hand, we always compared our results with those obtained from the normal band. But in the patients used for the present research, the opposite extremity was not infrequently in a condition of abnormal sensibility. We have therefore paid no attention to fine differences in precision, and, as will be seen from a perusal of our results, have dealt with large differences only.

The affected parts were also tested with a tuning-fork, and for this purpose we have used one beating 128 double vibrations a second. This is a treacherous form of stimalation, unless the patient is intelligent and the observer constantly on his guard. Error not uncommonly arises in consequence of conduction of the vibrations from an insensitive to some sensitive part at a distance; the patient then appreciates the stimulus, but not at the spot to which the fork was applied. It is most important that, among every set of stimulations, the fork sbould be from time to time

' It has been fully described in Brain, 1905, p. 228. 
applied when not vibrating, as patients occasionally answer "yes" when they appreciate only the pressure of its flat base, and not that sensation of vibration which is the essence of this test.

Ability to recognise objects of different sizes was tested by means of circular steel discs with diameters of $2 \mathrm{~cm}$., $0.75 \mathrm{~cm}$., $0.2 \mathrm{~cm}$. Of these, either two or three were applied indiscriminately to the part one after the otber, and the patient said "The first was larger than the second and the second was smaller than the third." If care was taken to apply these discs to the skin with approximately equal pressure, this simple method was found to work well in practice and gave better results than more complex procedures.

We have obtained no satisfactory results in pathological cases from any tests we have used to determine the power of appreciating the shape of an object applied to the skin. Zinc, indiarubber, wood and cardboard cut into simple figures, such as squares, circles and crosses, even when made to $5 \mathrm{~cm}$. in dianeter, could not be appreciated over the normal skin with sufficient certainty to be useful for clinical examination.

Sensibility to one form of pain was tested by means of a needle, or sharp pin, in the nsual way. Bat, in dealing with patients suffering from some lesion of the spinal cord, in whom sensibility to touch is frequently unaffected, it is particularly necessary to be certain that a correct answer ("prick") is accompanied by the appreciation of pain. For most of these patients can discriminate stimulation with the point from a touch with the head of a piu, although totally insensitive to the pain of a prick. This acuæsthesia is, in reality, a form of the appreciation of size, and is closely associated with the integrity of touch. If, for this resson, there was any doubt concerning the patient's power to appreciate the pain produced by a prick, a painful interrupted current was used as the test, and by this means it was easy to discover whether the patient's answers were based on the perfection of his acumsthesia or upon a true appreciation of pain.

The interrupted current can be used to test sensibility to 
touch or to pain, as was pointed out in a previous communication [13]. In this research we have used it mainly as a painful stimulus, and on such parts only as the palm have we employed the painless interrupted current.

Fortunately, fewer mechanical difficulties surround the testing with heat and with cold when the lesion lies within the spinal cord, than after injury to a peripheral nerve. For, if sensibility to either heat or cold is disturbed in consequence of au intramedullary lesion, the appreciation of intermediate degrees is disturbed, together with that of the extremes. We have, therefore, usually employed glass testtubes, but in some of our experiments have used silver tubes for the more delicate observations.

It is well to remember, when testing the scalp, that the hair insulates the skin, so that both heat and cold pass through with difficulty, and the results are liable to be unsatisfactory.

As tests for the extremes of heat and cold we have used ice and water at $50^{\circ} \mathrm{C}$. The temperatures selected for the intermediate degrees varied with the external temperature, and were in every instance applied to some normal part, such as the forehead of the patient and of the observer. Moreover, control tests were always made with a tube of a neutral temperature, neither hot nor cold.

One of the greatest difficulties, familiar to all who have investigated cases of diseases of the spinal cord, is the tendency of the patient to call all temperature-stimuli, whether hot or cold, by the same name. It, is most iuportant, under such circumstances, to interject frequent stimulations with a test-tube which is neither hot nor cold to the normal skin. Then it will be found that, of the two stimuli, the neutral tube is as frequently said to be hot or cold, as the tube which is hot or cold to the normal skin. ${ }^{1}$

The existence of a sensation of pressure was tested by means of a pencil, or other blunt object. After division of a peripheral nerve to the skin, whenever pressure is appreciated, its steady increase leads to the production of pain. But when the lesion is situated within the spinal cord this

' For one source of this fallacy, see p. 681 . 
is not the case, and it therefore became necessary to possess some means of measuring the amount of pressure capable of causing pain. For this purpose we have used a modification of Cattell's algometer, made for us by the Cambridge Scientific Instrument Company. The apparatus, as made in New York, consists of a cylindrical rod with a diameter of $75 \mathrm{~cm}$., which rans into a large handle. Pressure upon the free end of this rod compresses a spring, and the amount of this pressure can be read off from the movements of a marker on a scale let into the handle. The observer holds the instrument in his hand and presses the free end of the rod on to the skin of the patient, who cries out as soon as pain is produced. The amount of pressure applied is read from the beight of the marker on the scale. But we found that this instrument was liable to give faulty readings, due to mechanical difficulties in constructing an inverted balance that will read correctly. The modification we bave used was suggested by Dr. Rivers, who superintended the manufacture of our instrument. (Figs. 1 and 2.) There is no scale in the handle, which contains only the spring to be compressed. But on the rod slides a scale so arrauged that, when pressure is made and the rod is driven into the handle, the scale is pushed down upon the shaft of the rod.

Immediately the patient calls out that the pressure has become painful, the instrument is removed, the rod springs out of the handle again, carrying with it the scale, which remains at that point where the rod emerged from the handle at the moment of maximum pressure. A line drawn around the rod acts as an indicator, und the amount of pressure applied can be read off at leisure from the relation of this line to the measure on the scale. 'This scale is gruduated in kilograms.

An additional advantage obtained from this method of registration, is the ease with which the spring can be made to give constant readings. Such constancy is extremely difficult to obtain, if the spring is fitted with the necessary apparatus for obtaining readings by means of a marker on a scale.

We have found an algometer, constructed with these 


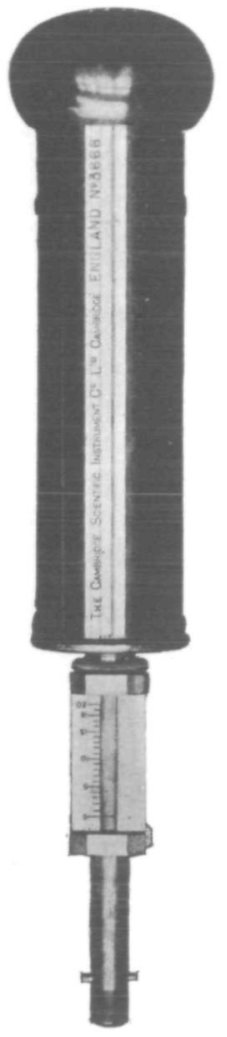

Fra. 1.

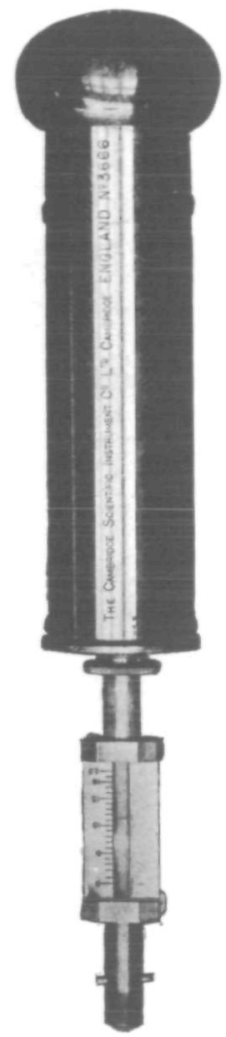

FTo. 2.

Fia. 1.-Shows the Algometer before use. The zero of the scale corresponds to the horizontal line on the rod.

Fie. 2.-Shows the Algometer after use. The horizontel line on the rod now corregponds to 11.5 divisions on the scale. At this point the patient complained that the pressure caused pain. The graduations correspond to kilograms. 


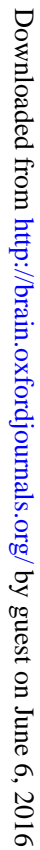


modifications, of the greatest use. It will give different readings in the hands of each observer on account of variation in the manner and rapidity with which the instrument is applied. We therefore neglect all small differences, paying attention only to those so gross that it is certain there must have been some grave defect in the patient's power of appreciating the pain of pressure.

We employed the following method for testing the power of recognising passive movements, and the position into which a limb had been placed passively. After closing the patient's eyes, the thumb, finger or great toe is grasped by the observer in such a way that his forefinger and thumb press on its lateral sspect. Then the finger or toe can be flexed or extended to a varying degree, without applying pressure in the direction of movement. When the wrist is to be flexed or extended, the movement is produced from one of the fingers, the arm being supported, so that the hand moves freely. Movement of the elbow is made by grasping the wrist laterally, and the knee is tested by supporting the thigh and flexing or extending the knee from the ankle. Thus every joint is moved by means of the one distal to it.

The patient is asked to say what movement is made and how the part under examination lies when movement is over. But we lay much more stress upon the results of the following test. $\mathrm{He}$ is askcd to imitate the movement with the linb of the opposite side. For instance, when the thumb of the right hand is under examination, every flexion, extension, or other movement, passively produced, should be followed by a similar voluntary movement of the thumb of the left hand. When both legs are affected, as is so commonly the case, in consequence of lesions of the spinal cord, the patient is asked to imitate with the thumb the movements of the great toe, and with the wrist those of the ankle.

Frequently, when in doubt, he will guess, and we therefore from time to time allow the part to remain quiescent in a definite position, still grasped between the fingers, after a movement has been made. Perbaps the answer given iy 
the patient has correspouded correctly with the movement made by the observer. But, in spite of the part remaining stationary in the same position, the patient indicates that it is being moved. This is one of the luost certain signs, either that be is untrustworthy, or that there is some grave defect in his power of recognising position assumed passively. Occusioually, when the patient's answers have been incorrect, we allow him to open his eyes, and his look and exclamation of astonishment on seeing the true position of his limb is strong evidence that his power of appreciating the position of the part is gravely diminished.

It is well to adopt a method of recording the answers similar to that used for the results of the compass-test. Teu movements are made in one direction, ten in the other, indiscriminately, and the results are marked above and below a line, each answer being recorded by a stroke if right, by a cross if incorrect. If no answer is given, an $\mathrm{O}$ is inserted, whilst doubt is indicated by a $\mathrm{D}$. We look upon this method as greatly increasing the accuracy of the record. (Cf. the records on p. 660).

Many methods have been used for testing the certainty with which a patient can indicate the point toucbed. For the purposes of this research we have used the following methods.

First, the patient, whose eyes were closed throughout the whole period of testing, was asked to uawe the part touched, pressed or otherwise stimulated. Secondly, he was told to point to the spot stimulated. In the case of the legs, he was given a cardboard rod to be used as a pointer. His eyes remained rigidly closed throughout the whole period of testing ("groping method",. Thirdly, a hole was cut in the centre of a stiff sheet of paper, and through this he was stimulated, the paper being held about $1 \mathrm{~cm}$. distant from the skin. He was given a soft pencil and with this tried to point to the spot stimulated. The point he reached was marked on the paper, and the distance from the central hole could be measured. (Spearman's method [31]).

In this research we have rarely used the method of Victor Henri [14], in which the patient is given a life- 
sized diagram of the part to be tested and is asked to mark upon it the point stimulated. Such a metbod is of great value when the hand is affected, a condition which seldom occurred in the cases brought forward in this paper.

Chapter II.

The nature of the loss of sensation produced by a lesion of the spinal cord, compared with that due to division of peripheral nerves.

Afferent impulses pass from the periphery to the spinal cord along three systems of fibres, those of protopathic, epicritic, and deep sensibility.

The protopathic system is essentially one of punctate sensibility. For heat, for cold and, probably, for pain, there are definite spots, from each of which a specific sensory impulse can alone be released. The hairs are also innervated partly from this system; and, when the skin is endowed with protopatbic sensibility only, exquisite pain is produced by plucking the hairs, and a curious diffuse tingling or formication occurs when they are brushed with cotton. wool.

Elimination of epicritic sensibility from any part of the body makes it impossible to distinguish two points of the compasses applied simultaneously, cutaneous localisation is perverted and light touch over hairless parts abolished. Whenever epicritic sensibility is destroyed, the power of appreciating intermediate degrees of heat and cold ("warm " and "cool") will also be lost. We believe that there is a separate cutaneous mechanism which reacts to temperatures we call warm $\left(34^{\circ} \mathrm{C}\right.$. to $40^{\circ} \mathrm{C}$.). It is also possible that coolness is due to impulses from end-organs other than the cold spots; the evidence for this view will form the subject of a further communication by one of us in conjunction with Dr. Rivers. But it is also couceivable that the sensation of "coolness" might be produced by the combined stimulation of this mechanism and the cold spots which 
belong to the protopathic system. According to this view an appreciation of "warmth" would be necessary for the production of the sensation of "coolness," and therefore destruction of epicritic sensibility would remove the power of appreciating the intermediate degrees of temperature, whether they normally give rise to a "warm" or a "cool " sensation.

The existence of both these forms of sensibility depends upon the integrity of fibres in the sensory nerves to the skin. But, even after the complete division of all nerves to the skin, the denervated part remains sensitive to pressure, and pain can be produced as soon as this pressure exceeds a certain measurable amount. Each pressure-touch can be localised with fair accuracy, and any movement of the joints is at once recognised. But no degree of temperature produces any sensation, and the part may be burnt or frozen with impunity; not even the pain of the extremes of heat and cold can produce a reaction in this system, which is so exquisitely sensitive to the pain of increasing pressure. The two points of the compasses applied simultaneously cannot be discriminated, but the vibrations of a tuning-fork beating 128 to the second can be perfectly recognised.

As this form of sensibility remains after all cutaneous afferent nerves have been destroyed, it is obvious that the fibres by which its impulses pass to the central nervous system must run, at any rate to a considerable extent, with the muscular nerves. But it must not be sapposed that we deny the existence of fibres in the superficial structures of the body, which belong to this deep system, and are capable of reacting to pressure in all forms.' Sherren, Rivers and Head were compelled to neglect them, because their only opportunities for investigating the properties of this deep system arose after division of all the nerves to the skin. But, in consequence of the peculiar dissociation of sensibility which we shall study in this paper, we see reason to believe that this system of afferent fibres running in great part with muscular nerves, also sends some fibres to the skiu, particularly of such parts as the palm of the hand and the sole of the foot. 
These statements can be summarised in the following table :-

\section{Loss of epicritic sensibility abolishes-}

Recognition of light touch over hairless parts or parts that have boen shaved.

Cutaneous localisation.

Discrimination of compass points

Appreciation of differences in size, including the accurate discrimination of the bead from the point of a pin apart from the pain of the priok (acursthesia).

Discrimination of intermediate degrees of temperature from about $25^{\circ} \mathrm{C}$. to about $40^{\circ} \mathrm{C}$.

Loss of protopathic sensibility abolishes-

Cutaneous pain, especially that produced by pricking, burning or freezing. together with that of stimulation with a painful interrupted ourrent. Over hair-cled perts, plucking the hairs ceases to be painful.

Sensations of heat from temperatures above about $45^{\circ} \mathrm{O}$.

Seasations of cold from temperatures below $20^{\circ} \mathrm{C}$.

After destruction of all cutanoous afferent fibres the part is still endowed with deep sersibility.

Pressure can be recognised, and its graduel increase spprecisted.

Pain is produced by excessive pressure (messured by the algometer).

Movements of muscles can be recognised.

The point of application of pressure can be looklised.

The patient can recognise the extent and direction of movement produced passively in all the joints within the affected area.

This grouping of the afferent impulses on their way from the periphery to the central nervous system is probably determined by reasons of phylogenetic development. But as soon as the first intramedullary cell-system is reached, these inpulses become shunted into paths along which they travel in new combinations, and in this chapter we shall discuss the forms assumed by the loss of sensation, due to a lesion interfering with afferent impulses, after this new grouping has occurrad.

\section{Section 1.-Pain.}

Nowhere is this difference of grouping more evident than in the behaviour of those impulses associated with the pain produced by deep pressure. After division of a peripheral nerve, or of posterior roots, those parts only become insens1tive to the pain of deep pressure which are at the same time totally insensitive to the tactile element of this stimulus. 
Unless all forms of deep sensibility are abolished, pain will still be caused by excessive pressure.

But, if the lesion lies within the spinal cord, sensibility to pain is abolished as a whole, whatever the form of painful stimulation. The patient will be insensitive both to the pain of excessive pressure and to that of a prick or of the interrupted current; yet, at the same time, he may be able to recognise the position of the spot pressed, and can appreciate the gradually increasing pressure.

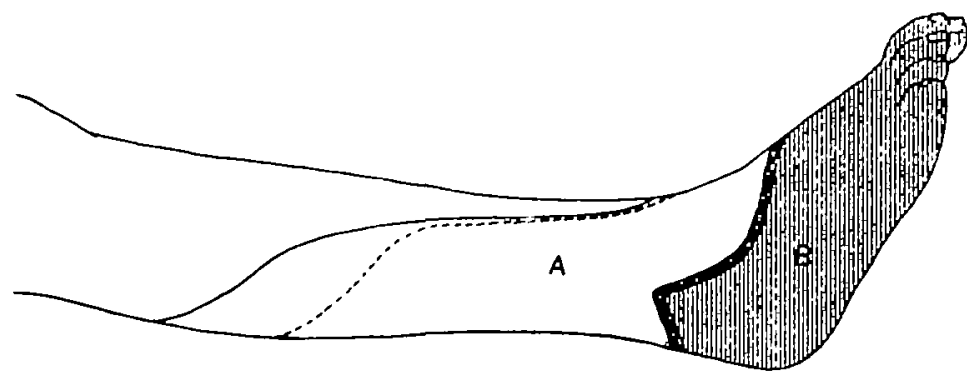

FIG. 3.

To show the loss of sensation in William B., produced by destruction of the eacral plexus.

The extent of the loss of all forms of sensation, including pressure, is shaded and marked $B$.

Those parts which were insensitive to all cutaneous painful stimulation but were sensitive to the pain of doep pressure are enclosed within a broken line and marked $A$.

Sensibility to light touch was lost below the single dark liue.

The followung case is an excellent instance of the condition produced by destruction of several nerve-tranks.

William B. (Originally reported by Sherren and Head [13], and later by Sherren [29]) was shot through the buttock on October 4, 1901. This produced loss of sensation over the region supplied by the great and by the small sciatic nerves, together with paralysis of all the muscles below the knee supplied by the latter nerve. He was seen by Mr. Sherren and one of us on several occasions from 1902 onwards. Finally, in May, 1905, he canue under the care of Mr. Sherren at the London Hospital, and at the exploratory operation the following condition was found. The sciatic plexus was divided below the point at which it was joined by the second sacral nerve ; 
the third sacral had been destroyed in the wound of the sacrum and the nerves were bound up in a dense mass of fibrous tissue. Mr. Sherren sutured the lumbo-sacral cord and the second sacral nerve to the stump of the sciatic. AB soon as the wound of this operation was healed, the sensibility of his leg was again tested and was found to correspond exactly to that before the operation. The extent of the loss is shown on Fig. 3, taken from a series of photographs. It will be seen that over the greater part of the foot, deep sensibility was lost (area $B$ ) ; here he was insensitive to the painful and tactile elements of pressure and falled to appreciate the. vibration of a tuning-fork. But on the outer aspect of the leg was an area of considerable extent $(A)$ over which he was entirely insensitive to the prick of a pin or to the pain of an interrupted current. Within this area, the same amount of pressure was painfal as on the sound side within similar limits. Thus a part of the leg completely insensitive to cutaneous painful stimulation was sensitive to the pain of excessive pressure. The only parts where pressure could not be made to produce pain, were those devoid of all forms of deep sensibility.

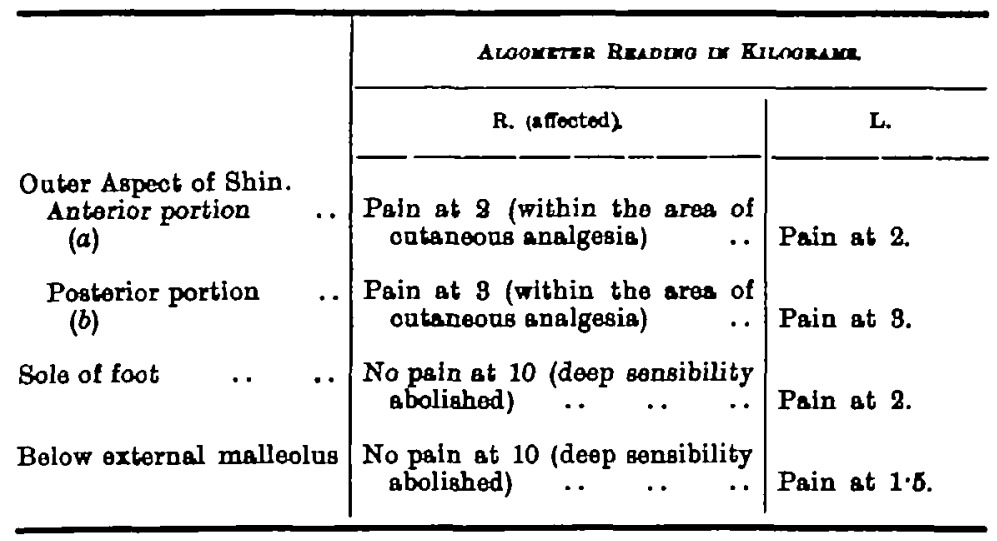

Compare with this dissociated analgesia the condition of the following patient (Case 17), who suffered from a lesion within the spinal cord. He was injured by the overturning of a truck of concrete on May 9, 1905, and was brought to

voL. XXIX. 
the London Hospital paralysed from the hips downwards. By May, 1906, he had partly recovered and showed a condition that was so sharp a contrast to that of the previous patient. (Fig. 4.)

He walked, dragging the left leg like a man with hemiplegia, and this leg was stiff and spastic. All movements could be performed by the various parts of the limb, but they were slow, especially dorsi-flexion at the ankle. Both knee-jerks were exaggerated, ankle clonus was obtained and the plantar reflex gave an extensor response on both sides.
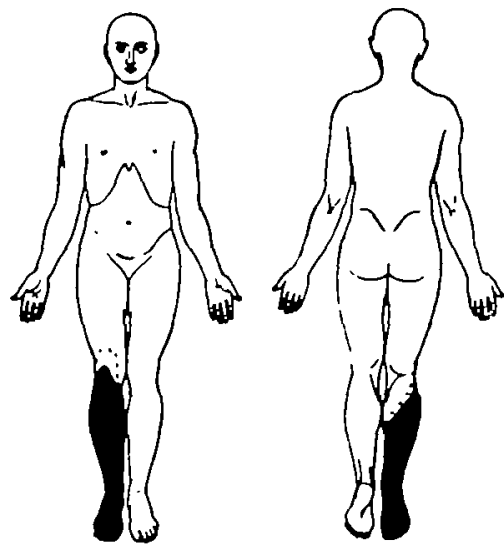

Fra.4.

To show the loss of sensation in Case 17, due to an injury to the spinal cord.

The derk area represents the parts insensitive to cutaneous painfal stimuli, and slso to the pain of excessive pressure; and yet over this ares light touch and the tactile element of pressure were appreciated.

The right leg below the knee was insensitive to prick and to all degrees of temperature. But, over the whole of this area, he could appreciate all tactile stimali and could localise accurately the spot touched or pressed upon. Yet it was not possible to produce pain anywhere over the right leg and foot by excessive pressure, although he fully recognised its gradual increase.

R.

$\mathbf{L}$

Outer aspect of leg $\quad \ldots \quad \ldots \quad \ldots \quad$. $\quad$ No pain at $16 \quad \ldots \quad$ Pain at 5 . Inner aspect of leg over tibia . . . . No pain at $10 \quad \ldots \quad$ Pain at 4. Front of thigh abore the limits ! $\quad \ldots \quad$ Pain at $5 \ldots \ldots$.. Pain at 5. 
Thus, in the former case, where the analgesia over the leg was produced by division of nerve-trunks, a large area of skin was sensitive to the pain of deep pressure, although insensitive to all painful cutaneous stimuli. Only over the foot, where deep touch evoked no response, was the pain of pressure abolished.

But, where the loss of sensation was produced by a lesion of the spinal cord (Case 17), the pain of pressure was absent over parts insensitive to prick, in spite of the complete integrity of the sensations of deep touch, and of the power of recognising the gradually-increasing pressure.

These two patients were chosen as examples, because the site of the loss of sensation was so nearly alike in the two cases. An equally striking contrast is given by

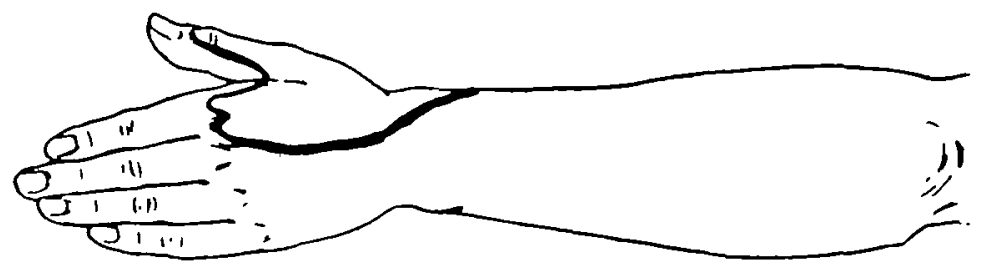

Fig. 5 .

To show the loss of cutaneous sensibility on the dorsum of the band produced by experimental division of the radial (ramas saperfioialis nervi radialis) and external cutaneous nerves in the neighbourhood of the elbow.

comparing the sensory condition of a part to which all the cutaneous nerves have been divided, with the loss of sensation produced by a pure lesion of the spinal cord.

On April 25, 1903, the radial and external cutaneous nerves were divided at the elbow in one of us. The area on the hand, which became entirely insensitive to all cataneous stimuli, including the pain of a prick and of the interrapted carrent, is shown on Fig. 5. But pressure was appreciated and, when increased, caused pain even more readily than over a similar part of the sound hand. The readings given by the algometer were as follows :-

\begin{tabular}{|c|c|c|c|c|c|}
\hline \multirow[b]{2}{*}{ Over the dorsum of the hand } & \multicolumn{4}{|c|}{ L. (affectod). } & R. (nound). \\
\hline & .. & $\ldots \quad 3.5$ & .. & .. & $3 \cdot 5$ \\
\hline In flrst interosseous space & . & $\therefore \quad\left\{\begin{array}{l}2.0 \\
20\end{array}\right.$ & $\begin{array}{l}\cdots \\
\cdots\end{array}$ & .. & $\begin{array}{l}3.5 \\
4.0\end{array}$ \\
\hline
\end{tabular}


Compare with this dissociated sensibility to painful stimuli, the sensory condition of the hands in Case 13, a patient suffering from syringomyelia. The whole of the area marked on Fig. 6 was insensitive to prick and to the painful interrupted current. Tactile sensibility was everywhere perfect; he could localise accurately the spot touched, or pressed apon, and appreciate passive movements of all the joints. But pain could not be produced by pressure anywhere within the area of cutaneous anal-

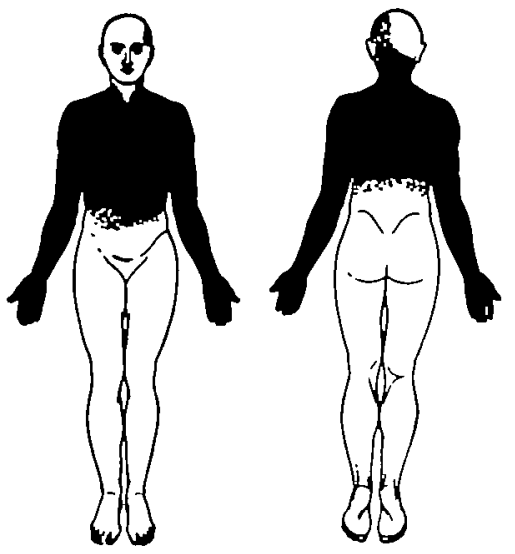

Fig. 6.

To show the loss of sensation to cutaneous painful stimuli in a case of syringomyelia (Case 13). The whole of the area marked black was insensitive to the pain of excessive pressure.

gesia, although he recognised the gradually increasing stimulation.

R.

Palm of hand .. $\quad$.. $\quad$. $\quad$ No pain at $15 \quad \ldots \quad$. $\quad$ No pain at 15.

Thenar eminence $\quad \ldots \quad \ldots \quad$ No pain at $15 \quad \ldots \quad$.. $\quad$ No pain at 15.

Sole of foot $\quad . . \quad \ldots \quad$.. $\quad$. $\quad$ Pain at 4 .

Thus, in conclusion, we bave found that, when sensibility to pain is abolished in consequence of an intramedullary lesion, all forms of painful stimuli are simultaneously affected. But, if the lesion be situated in the peripheral nerves or posterior roots, the painful impulses produced by excessive pressure are not interrupted, unless deep seusibility is abolished as a whole. 
Section 2.-Heat and Cold.

Great as is the contrast between the grouping of painful afferent impulses in the peripheral and central nervous system, those for sensations of heat and cold undergo an even more complete redistribution. Destruction of either the epicritic or the protopathic fibres interferes with the afferent impulses, both for beat and for cold. If epicritic sensibility is abolished, the patient cannot discriminate intermediate degrees of beat and cold, but remains sensitive to the more extreme temperature stimuli. After \& peripheral nerve has been divided, the protopathic system recovers first; this recovery endows the part with sensibility to cold below $20^{\circ} \mathrm{C}$. and heat above about $40^{\circ} \mathrm{C}$.

Injury to peripheral nerves or posterior roots abolishes, to some degree, sensibility both to heat and cold; recovery restores to the part sensibility to both forms of thermal stimulation.

But, when the lesion is sitnated within the spinal cord, areas may be found insensitive to heat but sensitive to cold, or vice vers $\hat{a}$. An intramedullary lesion may even destroy all sensibility to one form of thermal stimulation, leaving the parts completely sensitive to the other. Evidently the whole arrangement of thermal impulses has undergone a change within the spinal cord. All impulses which subserve sensations of heat, whether they arrive by way of the epicritic or protopathic systems, become a single intramedullary group. In a similar manner all thermal impulses, whatever their source, which can produce sensations of cold, are brought together within the spinal cord.

G. G. A. (Case 8) was a good example of this condition. Sensation was disturbed down the right half of the trank and over the right leg, whilst the left leg was stiff and spastic. The right knee-jerk was brisk, but the left was exaggerated, and clonus was obtained from the left ankle. He was analgesic below the level of the nipple on the right side (Fig. 7). Cold was appreciated everywhere above the right knee; but the outer aspect of the right leg and the whole of the right foot were insensitive to stimulation by cold in all degrees (Fig. 8). Heat from $35^{\circ}$ C. to $65^{\circ} \mathrm{C}$. 
was everywhere appreciated correctly, except that, over the parts insensitive to prick, he did not experience the usual sensation of burning pain when the temperature rose above $50^{\circ} \mathrm{C}$; and yet he could clearly appreciate the increasing heat when the stimulus changed from $50^{\circ} \mathrm{C}$. to $60^{\circ} \mathrm{C}$.

Here no cold stimulus produced a sensation over parts of the leg, where all temperatures from $30^{\circ}$ to $60^{\circ} \mathrm{C}$. caused a sensation of heat. Such a total dissociation of the sensations of heat and cold never occurs from division of peripheral nerves.
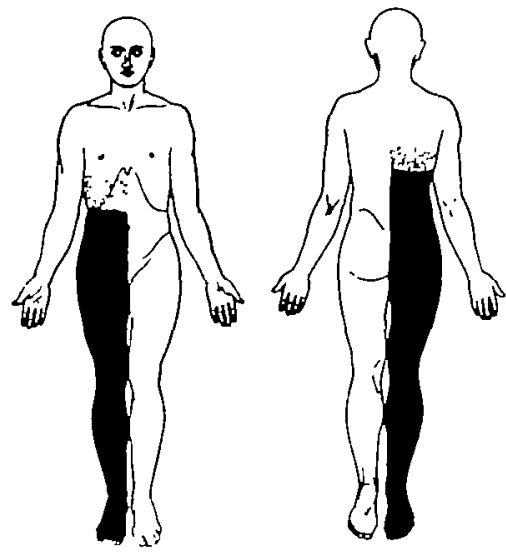

Fra. 7.
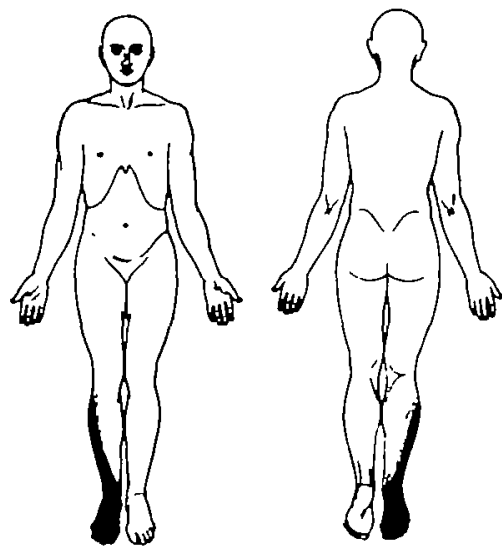

FIG. 8.

A similar dissociation of sensibility to beat and cold, but in the reverse direction, was seen in Cyril W. F. (Case 7). In consequence of an injury to the neck, due to diving into shallow water, this young man produced weakness of the right arm, accompanied by loss of sensibility to pain, heat and cold over the left half of the body, each area of loss of sensation having a different extent (Figs. 9 and 10). From the level of the ensiform cartilage downwards to the penis, he was insensitive to all degrees of heat only; temperatares between $30^{\circ}$ and $60^{\circ}$ caused no sensation of warmth. But, 
over this area, he was sensitive to all cold stimuli, recognising them at once.

Here, again, the sensibility to heat and to cold were dissociated. Moreover, those parts insensitivs to heat were insensitive to all degrees from $30^{\circ} \mathrm{C}$. to $60^{\circ} \mathrm{C}$, a condition which never occurs from any lesion of peripheral nerves.

In the case of Mrs. G. (No. 3), the loss of sensation to various thermal stimuli was tested with extreme care on many occasions, and she was found to be insensitive on the left leg to all degrees of temperature between $0^{\circ} \mathrm{C}$. and $60^{\circ} \mathrm{C}$.
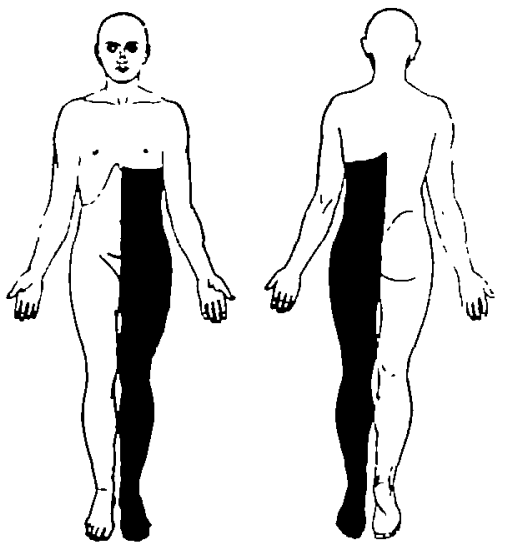

Fia. 9.
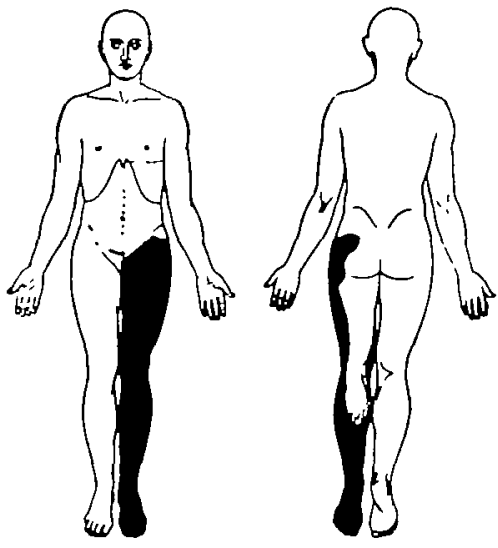

Fia. 10.

To show the loss of sensation to hest (fig. 9) and to cold (fig. 10) dae to injury to the cervical spinal cord (Case 7).

As she was also insensitive to pain over the same parts, it might have been urged that in this case both protopathic and epicritic sensibility had been abolished. The falsity of such a supposition is at once evident from the fact that she was everywhere sensitive to light touch, and that the compass test gave normal results over the affected areas. This condition can never occur from a lesion of peripheral nerves or posterior roots.

An exactly similar loss of sensation to all grades of heat and cold appeared in Case 2, in consequence of an injury to the cervical spine. This completely abolished sensibility to 
pain, heat and cold over the left leg and left half of the body, leaving all forms of tactile sensibility entirely unaffected.

In conclusion, we find that the afferent impulses produced by thermal stimuli are differently grouped in the peripheral nerves and in the spinal cord.

(1) Sensibility to heat may be abolished without coincident disturbance of that to cold, and vice versa.

(2) When sensibility to hest is disturbed in consequence of an intramedullary lesion, the patient no longer appreciates any thermal stimulus between $30^{\circ} \mathrm{C}$. and $60^{\circ} \mathrm{C}$. That is to say, insensibility is absolute to both intermediate and extreme degrees.

(3) Insensibility to heat and cold may be absolute, and yet the patient may be able to recognise the lightest tactile stimulation and to discriminate the two points of the compasses. Such conditions can never occur from a lesion of peripheral nerves only.

\section{Section 3.-Light Touch and Deep Touch.}

One of the most characteristic features of the loss of sensation following division of peripheral nerves, is the frequency with which light touches are not appreciated, although deep touch and pressure are immediately recognised.

But, when the lesion is purely intramedullary, all these sensory impulses come and go together. Thus, in a characteristic instance of Brown-Séquard paralysis (No. 1), the patient was unable to recognise either light, touch or pressure, applied within the affected area on the right half of the body. The whole of the right leg was insensitive to stimulation with cotton-wool. No reaction could be obtained to any of von Frey's hairs, although No. 5 (cf. p. 542) was promptly recognised over the left leg. Pressure, even of many kilograms, produced no sensation, unless the position of the muscles was shifted. Yet this patient recognised accurately any alteration in position, or any lnovement produced passively in the insensitive leg. Such a condition cannot possibly arise from a lesion of peripheral nerves. 
Thus, whenever sensibility to touch is abolished on the opposite half of the body, in consequence of an intramedullary lesion, we believe that all forms of tactile stimuli will be found to be affected. The peripheral afferent impulses for touch and pressure, arriving by way of the epicritic and deep systems, become combined in the spinal cord, so that the lightest perceptible touch produces a minimal tactile impulse, which passes up the spinal system among the tactile impulses evoked by the severer stimulus of pressure. ${ }^{1}$

\section{Section 4.-Passive Positiols and Movement.}

After division of peripheral nerves, the recognition of passive movement, and of the position into which any part of the limb has been placed passively, is strikingly associated with the integrity of that system of nerves concerned with the maintenance of deep sensibility. So long as only the cutaneous afferent nerves are divided, the patient can appreciate all movements of the joints, is sensitive to pressure, and complains of pain when this pressure is increased. There is a close association in the peripheral nervous system between sensibility to.pressure, the pain of excessive pressure and the power of recognising passive movement and position. All three forms of sensibility are present or absent together after lesions of the peripheral nerves, and all three depend upon the integrity of those afferent fibres which run with muscular nerves.

With an intramedullary lesion, on the other hand, all recognition of the position assumed passively by a limb is entirely dissociated from the afferent impulses produced by pressure. Both groups are equally dissociated from those impulses which underlie the recognition of the painful nature of excessive pressure.

In fact, Case 1 shows that the patient may be able to appreciate passive position and movement, although totally insensitive to every other sensory stimulus. This man was suddenly seized with loss of power in the left hand and leg, accompanied by absolute loss of sensibility to pain, heat

'For a farther consideration of the relation between sensations of light and deep touch, $c f$. p. 575 . 
and cold over the opposite half of the body. His power of movement was gradually restored, but the loss of sensation remained. Insensibility to touch and pressure accompanied the analgesia and thermo-anæsthesia, and excessive pressure produced no pain. But, in spite of this profound loss of sensation, the sense of passive position and movement remained undisturbed in both lower extremities. The most careful testing failed to show a difference between any parts of the two legs in the power of recognising movement or position passively assumed. Here, in spite of the absence of all sensibility to deep touch and pressure, the sense of passive position was not disturbed.

In the case of James Y. (No. 10) an exactly converse condition was present; sensibility to touch and pressure was present, but he was unable to recognise the position into which his limbs had been placed, and failed to perceive even the most extensive movement. In consequence of a metastatic growth within the spinal cord, this man bad rapidly lost power in both legs. When seen by us a month after the onset, both legs were flaccid and paralysed, and sensibility to painful and thermal stimuli was abolished below the knees and diminished over the area shown in Figs. 11 and 12 ; light touch and pressure could be recognised everywhere. But he was unable to tell the position into which his legs had been placed; he could not recognise even the grossest movements at the hip, the knee or the ankle. With this loss of appreciation of passive position and movement was associated a complete absence of discrimination of two points, even when separated to a distance of $20 \mathrm{~cm}$.

This dissociation of the sense of passive position from the impulses of all other forms of sensation, except the discrimination of two points, is peculiarly well illustrated by the case of Charles B. (No. 2). At the time he was first seen, motion was impaired in the left leg, and sensibility to pain and temperature abolished in the right leg and right half of the body. He could appreciate passive mover.ent and the position of all his limbs perfectly. But, in conse- 
quence of the operation for the removal of the fractured portion of the spine, he lost the power of recognising passive movement and position of the left leg, i.e., the leg in which all other sensory functions were perfect.

Such loss of the sense of passive movement and position on the opposite side of the body to the analgesia and thermo-anæsthesia is the rule in Brown-Séquard paralysis, and is illustrated by several of our cases.

In conclusion, we have been able to show that, in consequence of an intramedullary lesion, every other form of

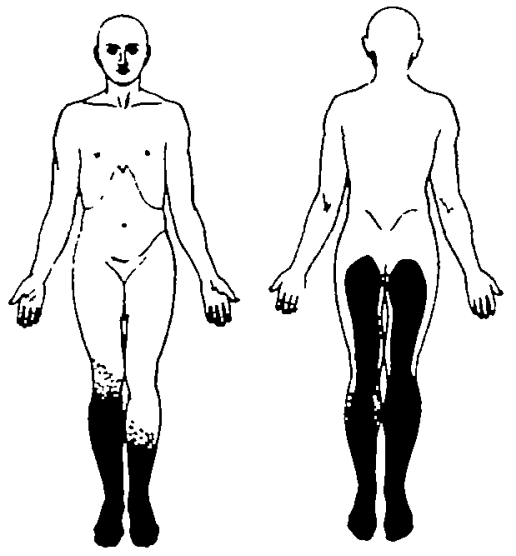

Fig. 11.
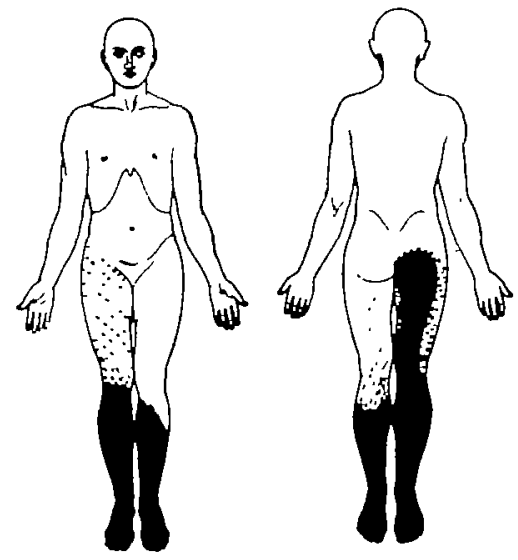

Fia. 12.

To show the loss of sensation produced by a metastatic growth within the spinal cord in Case 10.

Fig. 11. Shows the extent of the loss to pain.

Fig. 12. Shows the extent of the loss to hest and cold.

The extent of the lose of sensation to painfal and thermal stimuli varied considerably from day to day, and the areas of total loss were bounded in every case by parts of diminished sensibility.

sensibility may be abolished in a part which still remains sensitive to passive movement. (Case 1.)

Secondly, we have shown that the patient may be unable to recognise even the grossest passive movements, and be entirely anaware of the position of his legs, although they are sensitive to all tactile and pressure stimuli. (Case 10.)

Thirdly, we can bring forward a number of instances where the sense of passive position and movement was dis- 
turbed in one leg, whilst every other loss of sensation was to be found in the leg of the opposite side. (Case 2.)

Such dissociation of the impulses underlying the recognition of passive position and movement from the impulses of the other forms of deep sensibility cannot occur in consequence of injury to peripheral nerves.

\section{Section 5.-The Compass-Test.}

One of the most remarkable differences between the loss of sensation produced by division of a peripheral nerve and that due to a lesion within the spinal cord is revealed by the compass-test. When a nerve has been injured, sensibility to light touch is the first to be disturbed; with this failure to appreciate careful stimulation with cotton-wool over hairless parts, the compass-test will be invariably found to give resalts considerably below the normal accuracy. In fact, from the point of view of the peripheral nervous system, the compass-test is an accurate measure of tactile sensibility of the skin. For this purpose Head and Sherren [13] found it most useful, when carried out by the modified method described in their paper.

But, when the lesion is situated within the spinal cord, sensibility to light touch and the power of discriminating two points may be strangely dissociated.

In order to demonstrate this characteristic dissociation, all forms of tactile sensibility must be preserved. For it is obvious that, if the part to be tested is insensitive to touch and pressure, the compass-test will fail from want of afferent impalses. A diminution of tactile sensibility will produce a less accurate compass record, not because the special power of discriminating two points is lessened, but on account of the diminished acuity of the tactile impressions that are to be discriminated. [Case 4, p. 666.]

We have, therefore, selected those cases only where all forms of tactile sensibility were perfect, and yet the power of discriminating two points was gravely disturbed.

In Case 10, a man, aged 61 years, had become rapidly paralysed in both legs in consequence of a growtb within the 
spinal cord [Figs. 11 and 12.] With this paralysis, he had lost the power of appreciating the position of bis limbs to an unusual degree, and was unable to discriminate the two points of the compasses when widely separated. Over the external surface of the leg, he failed entirely at $15 \mathrm{~cm}$. and at $20 \mathrm{~cm}$. over the front of the thigh; over the palm of the left hand, a part unaffected by paralysis of motion or sensation, he gave correct answers at a distance of $1.5 \mathrm{~cm}$. At this time, there was no part over which he did not at once respond to light touches, or to pressure, and this grossly defective power of discriminating two points cannot, therefore, have been due to altered tactile sensibility.

This dissocistion is shown in a peculiarly striking manner by Case 16 . The patient, a man, aged 32 years, had suffered for four years from symptoms of a growth within the cervical portion of the spinal cord. This produced a loss of sensation over the arms and trank shown on Figs. 13 and 14 ; but both legs remained sensitive to all painful, thermal and tactile stimuli. With the finest tests, no difference could be discovered in the tactile sensibility of the two legs. With von Frey's bairs, No. 5 (cf. p. 542) could be appreciated everywhere over the soles of both feet; No. 4 produced no sensation on either limb. And yet the power of discriminating two points was gravely diminished over the left foot and leg. Over the sole of this foot, be answered badly when the points were separated to a distance of $8 \mathrm{~cm}$.

$$
8 \mathrm{~cm} \cdot \frac{1 \mid 10 \mathrm{R} .}{2 \mid 5 \mathrm{~W} .5 \mathrm{R}}
$$

whereas, over the right sole, his answers were perfect at $4 \mathrm{~cm}$.

$$
4 \mathrm{~cm} \cdot \frac{1 \mid 10 \mathrm{~B} .}{2 \mid 10 \mathrm{R}} \text {. }
$$

Over the outer aspect of the left leg, he failed with the points separated to $15 \mathrm{~cm}$.

$$
15 \mathrm{~cm} \cdot \frac{1 \mid 4 \mathrm{~W} .6 \mathrm{R}}{2 \mid \mathrm{SW} .5 \mathrm{R} .} \text {. }
$$

but over a similar part of the right leg, his answers were uniformly correct at $8 \mathrm{~cm}$. 
These two cases show that, within the spinal cord, the impulses which underlie the power of discriminating two points are separated from those of tactile sensibility. Having passed up in the epicritic system, they become dissociated in the spinal cord from the remaining forms of epicritic sensibility. Within the spinal cord, they pass on in close association with the impulses which underlie the recognition of passive movement and position of the limbs, impulses which have reached the cord by means of the system of fibres subserving deep sensibility.
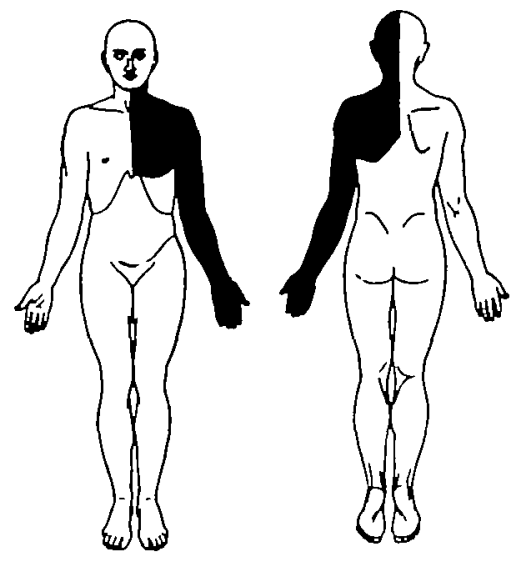

Fia. 19.
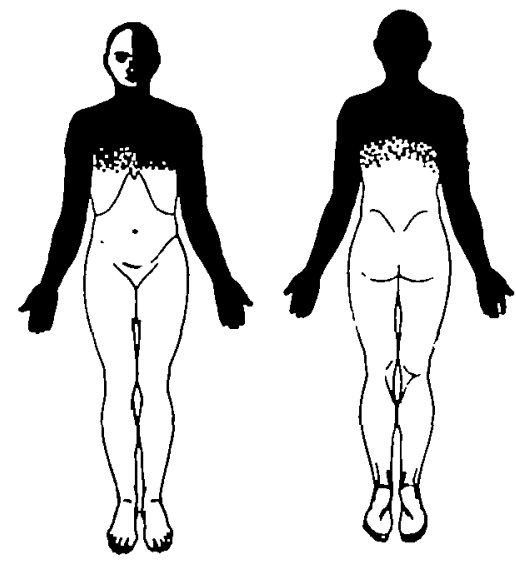

Frg. 14.

To show the loss of sensation in a case of disease of the medulla oblongata and cervical portion of the spina] cord [Case 16.]

Fig. 13. Shows the loss of sensetion to painful stimuli. Fig. 14, the loss to heat. To cold, the anæs thesia was slightly less extensive [vide p. 731].

The only loss of sensetion in the lower extremities consisted of profound loss of the sense of passive position and movement, snd of defective sensibility to the compass-test in the left leg.

Chapter III.

The Inter-Relation of Afferent Impulses in their Passage up the Spinal Cord.

In the previous cbapter, we have shown that afferent impulses, which reach the central nervous system by way of the peripberal ncrves, undergo rearrangement within the spinal cord. We shall now attempt to trace their course 
upwards, indicating as far as possible the relation of each set of impulses to the others.

More than fifty years ago, Brown-Séquard stated that division of one balf of the spinal cord caused loss of sensibility to pain on the side opposed to the lesion, and that motion was affected on the same side.

Led by the results of his experiments on animals, he examined more carefully the condition of sensation in patients suffering from what was called spinal hemiplegia. He attempted to show that this condition was the result of disease or of injury to one half of the spinal cord, which produced paralysis of voluntary movement on the one side, and anæsthesia on the other side of the body.

"Experiments on animals and a great many clinical facts," he says [7], "show that an injury, destroying a small zone, or dividing the whole lateral balf of the spinal cord in the cervical region, produces the following symptoms:-

"1st.-Paralysis of the voluntary motor conductors on the same side.

" 2nd.-A paralysis also of the vasomotor conductors on the same side, and, as a consequence, a greater afflax of blood and a higher temperature. . . . . .

" 5 th.-There is anæsthesia of all kinds of sensibility, excepting the muscular sense, in the side opposite to that of the lesion in the spinal cord, owing to the fact that the conductors of sensitive impressions from the trunk and limbs decussate in the spinal cord, so that an injury in the cervical region of that organ in the right side, for instance, alters or destroys the conductors from the left side of the body.

" 6 th.- There is some degree of anæsthesia also on the side of the lesion, in a very limited zone, above the hyperæstbetic parts, and indicating the level of the lesion in the cord. This anæsthesia is due to the fact that the conductors of sensitive impressions reaching the cord through the posterior roots, at the level or a little below the seat of the alteration, have to pass through the altered part to reach the other side of the cord."

This admirable summary is followed by the narration of 
two cases, observed by Brown-Séquard himself, with a fulness rarely equalled in the present day [7].

His results have been attacked from two points of view. It was pointed out that the lesions produced by disease were crude and ill defined, and commonly affected both halves of the spinal cord. Moreover, post-mortem examinations on those who had suffered from Brown-Séquard paralysis are uncommon, except in cases complicated by grave general disease, which renders difficult an accurate investigation of sensibility.

Physiologists attempted to solve the question by means of animal experiments, carried out with all modern surgical precautions. Some (especially Turner [37j) supported the original view of Brown-Séquard, others (Schiff, Mott [19], Rothmann [24], Schuster [26]), conchuded that afferent impulses did not cross in the diagrammatic manner he described. Moreover, many experimenters have shown that hemisection in the dorsal region produces ascending degeneration in both the same and the opposite half of the spinal cord. It is therefore certain that to afferent impulses, arriving by way of the posterior roots, both crossed and uncrossed paths are open.

But of the nature of the afferent impulses passing by these paths, experiments on animals can tell us little. It is difficult to conclude whether they are non-sensory afferent impulses, concerned with what may be called the higher reflexes, or whether they are ultimately concerned with some form of sensation. For every examination of sensation is in reality a psycho-physical experiment, where the extent of the mental error can entirely obscure the physical result. The nature and extent of these errors we can estimate only in man. Moreover, it is not sufficient to note the response to a stimulus; we must hear from the lips of the patient his experiences under stimulation. Supposing he starts and withdraws his foot when pricked, we cannot conclude that he experiences pain. It may be that his withdrawal is the reflex response to pressure with a pointed object. He alone can tell us the nature of his sensations. (Compare p. 644.) 
Thus, we are compelled to fall back upon the results of disease. But the destruction produced by disease is greatly wanting in precision compared with an experimental lesion. Moreover, on account of lack of time, or forethought, or the progressive nature of the disease, many clinical records are wanting in necessary fulness and precision. Thus, on the one hand, are those who, for the sake of the definiteness of the lesion, cling to the results of animal experiment, which can demonstrably lead to elementary conclusions only concerning sensation; on the other stand the clinicians, with their imperfect records and ill-defined terms.

We do not attempt to base our conclusions on the verification of the position of the lesion by post-mortem examination. For, in most cases where a microscopical examination was possible, the general condition of the patient had been unfavourable to that rigid and repeated examination by which alone the condition of sensibility can be established.' We shall assume that an intramedullary lesion disturbs the motor functions, and alters the reflexes on the same side of the body. This no one bas doubted. Then we shall examine the effect produced by an intramedullary lesion upon the patient's snswers to the series of sensory tests detailed in Chapter I. From these we shall deduce the manner in which the sensory impulses are grouped in their passage up the spinal cord, and correlate the loss of sensation with the motor disturbance.

Such study of the nature and relation of sensory impulses, when disturbed by an intramedullary lesion, bas led to more fruitful results than the anatomical method, which demands in every instance that the lesion should be demonstrated to be unilateral. For a complete unilateral lesion, especially if it be the result of progressive disease, will certainly cause functional and even microscopical changes in the opposite half of the cord.

We have arranged on Table 1 (p. 570), eight cases of Brown-Séquard paralysis observed by ourselves. In every instance the motor distarbance was limited to the one half

\footnotetext{
1 Wo have rejected two cases in which a complete microscopical examination was made on account of faulty sensory records.
}

vOL. XXIX. 


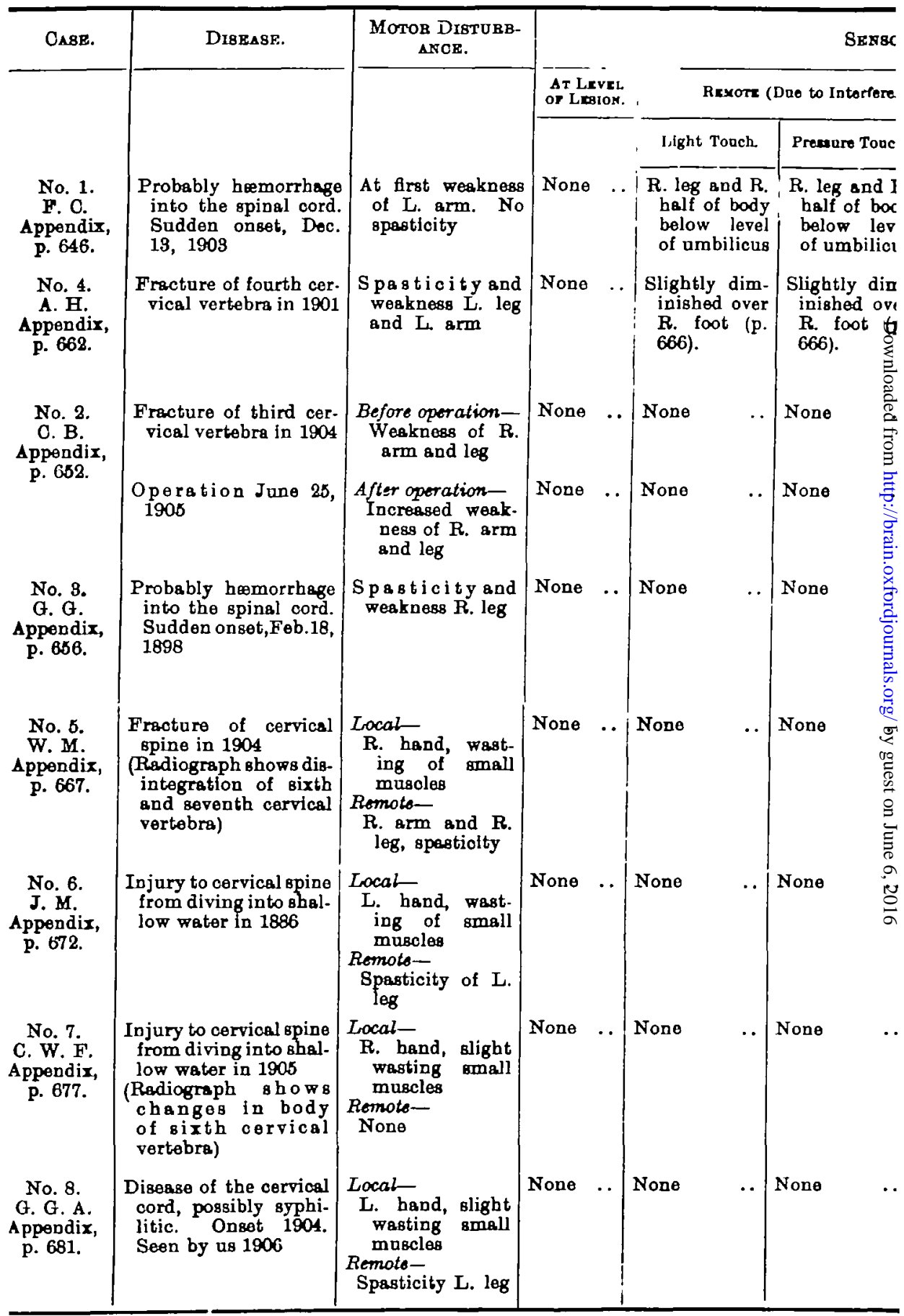




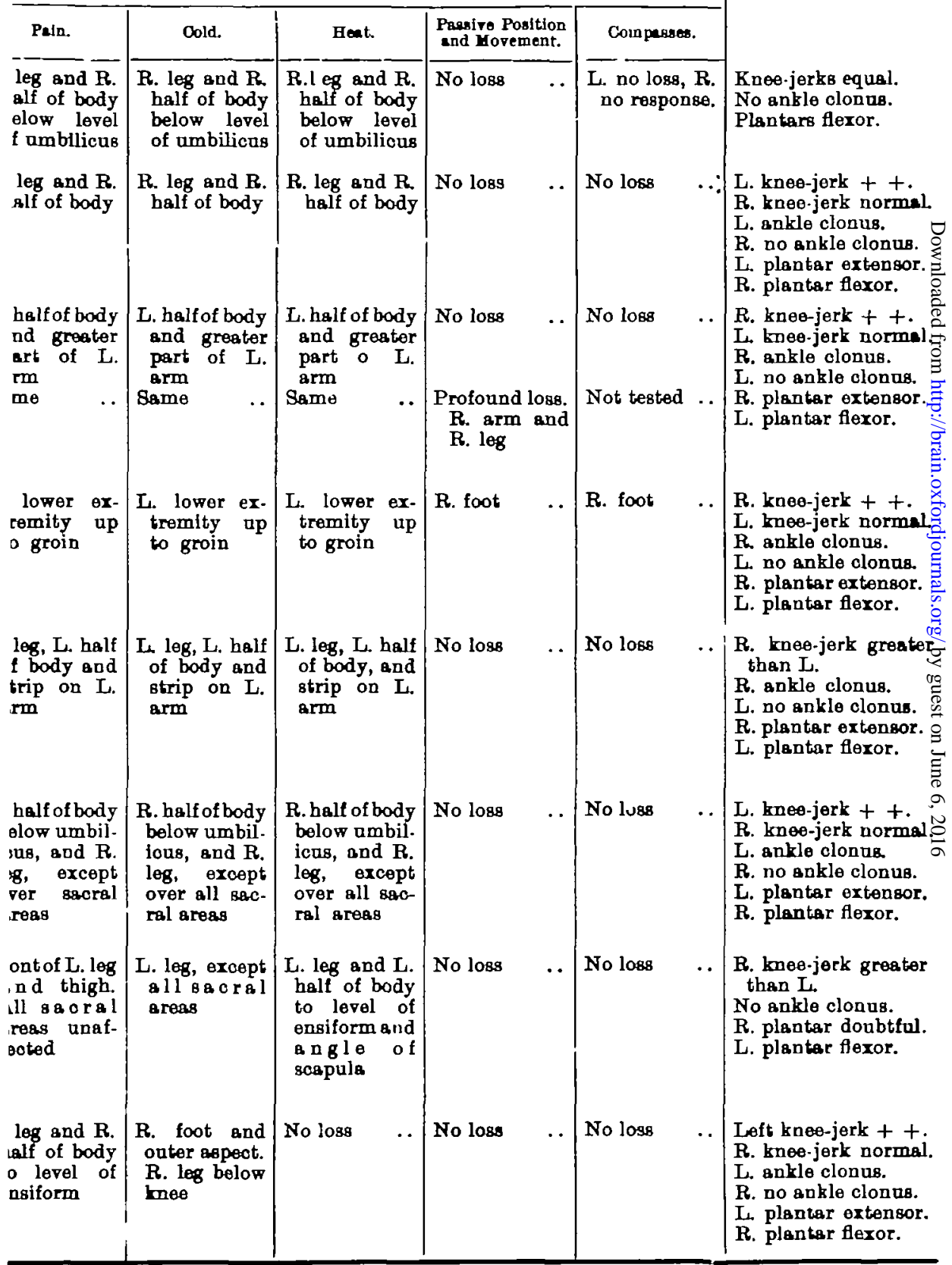


of the body, and in no case was the same form of sensation disturbed on the two sides.

In this chapter we shall deal with the disturbances of sensation remote from the level of the lesion. As BrownSéquard pointed out, a lesion of the spinal cord not uncommonly produces some degree of anmsthesia on the side of the lesion, due to an interruption of sensory impulses at the level of the disease. These local manifestations will be reserved for Chapter IV., while in the present chapter we shall deal with the disturbances of sensation in parts remote from the level of the lesion.

\section{Section 1.-Pain, Heat and Cold.}

The close relation between the impulses for sensations of pain and temperature bas long been universally recognised. Easily investigated and clearly defined, the loss of sensibility to pain, heat and cold is well described in most cases of disease of the spinal cord, however insufficient may be the remainder of the record. Petrén [21] has gathered together records of 137 unilateral lesions of the spinal cord in a monograph on this subject. He concludes that, in 128 cases where the condition was adequately recorded, sensibility to pain, heat and cold was abolished on the side opposite to the lesion.

Thus, whatever may happen to the impulses which underlie other forms of sensibility, there can be little doubt that, in man, those for pain, heat and cold cross to pass up the opposite side of the spinel cord.

To these cases may be added the eight instances of Brown-Séquard paralysis in the Table on p. 570. In all it will be found that the motor distarbance was strictly anilateral, and the remote loss of sensation was confined to one side; in all, sensibility to pain, heat and cold was disturbed on the side opposed to that of motion.

We have already described the character of this loss of sensation in Chap. II. When sensibility is abolisbed to pain, from an intramedullary lesion, no normally painful stimalus, whether it be a prick, the interrupted current, or excessive pressure, will produce a painful afferent 
impulse. In the same way, sensation is lost to all degrees of heat or of cold, except at the edges of an ill-defined area of thermo-anæsthesia. Here the more extreme degrees of heat or of cold may produce a sensation, because they are a more intense thermal stimulus than the intermediate degrees ( $c f$. Piltz [22]); but, if the insensitive area has welldefined borders, all forms of the specific sensibility to heat or to cold are abolished altogether.

Although the impulses for pain, heat and cold are so closely associated in their passage up the spinal cord on the side opposed to their point of entry, they do not travel inextricably intermingled. Not only can the three forms of sensibility be destroyed over different skin areas, but it is possible that one may even escape entirely.

In Case 8 , it is almost certain that at one time sensatious of pain, heat and cold were lost over the right half of the trunk and over the right leg. But, when the patient was first seen, the dissociation was pronounced. He could not appreciate a prick, or the pain of excessive heat, or the painful faradic current, over the right balf of the body below the costal margin. But sensibility to all degrees of cold was lost over the right leg below the knee only, whilst temperatures between $35^{\circ} \mathrm{C}$. and $65^{\circ} \mathrm{C}$. could be everywhere recognised. (Figs. 7 and 8. )

It is particularly in lesions of the cervical portion of the spinal cord that the three forms of sensation tend to be unequally affected. In August, 1905, C. W. F. (Case 7) injured his neck from diving into shallow water; when seen by us in November, the loss of sensation was more extensive to heat than to cold, to cold thau to pain (Figs. 15, 16, 17). Moreover, this patient showed another phenomenon not uncommon in lesions of the apper part of the cord ; the analgesia occupied the front of the thigh and leg, but did not extend on to the sacral areas. To a less degree, this was the case also with the loss of sensation to cold. Thus, a lesion in the cervical region abolished sensation over the opposite leg, without interfering with the impulses from the terminal portions of the spinal cord. This was extremely well shown in the case of J. M. (No. 6), who twenty years before we 
first saw him, had injured his cervical spine by diving into shallow water. In this instance, the extent of the loss to pain was greater than that to cold and to heat.

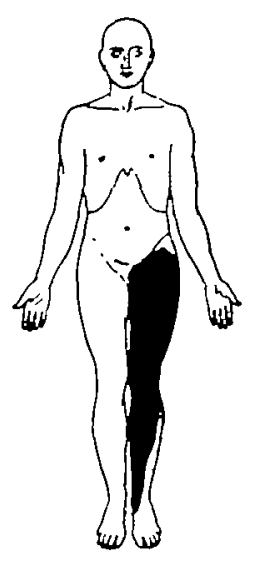

Fio 15.

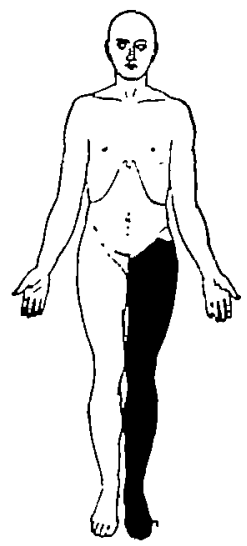

FIG. 16.

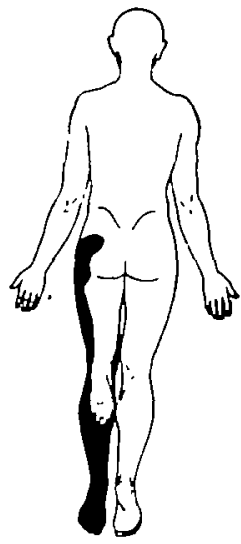

0
0
0
0
0
0
0
0
0
0
0
0
0
0
0
0
0
0
0
0
0
0
0
0
0
0
0
0
0
0
0
0
0
0
0
0
0

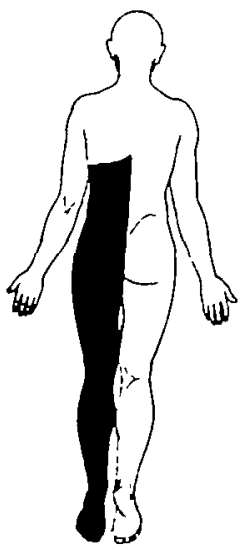

Fig. 17.

To show the loss of sensstion in Case 7.

Fig. 15 shows the extent of the loss of sensation to painfal stimuli.

Fig. 16 shows the loss to cold.

Fig. 17 shows the loss to hest.

When the loss of sensation produced by an intramedullary lesion occupies the opposite half of the body only, the 
boundary between parts of normal and abnormal sensibility is usually ill defined. This might have been foretold from our knowledge of the irregular distribution of such lesions.

But, occasionally, the upper border of the loss of sensation to pain, to heat and to cold can be determined with unusual certainty. Such definiteness of outline must signify that all impulses passing upwards have been interrupted completely at a certain level.

Now it would seem from some of our cases that, even when the impulses for pain, heat and cold are interrupted after they have crossed the cord, the situation and shape of the border between parts of normal and abnormal sensibility point to a segmental arrangement. (For a full consideration of intramedullary segmentation vide Chap. V.).

In conclusion, we believe :-

(1) That afferent impulses for pain, heat and cold cross the spinal cord to pass up the opposite side.

(2) That the remote loss of sensation may affect to a varying degree the impulses for the three forms of sensibility.

(3) The borders of this remote loss of sensation on the opposite side of the body to the intramedullary lesion usually merge gradually into parts of normal sensibility. But, occasionally, they correspond to the borders of intramedullary segments.

\section{Section 2.-Touch and Pressure.}

In a previous chapter, we showed that the disturbance of tactile sensibility, due to an intramedullary lesion, differed fuudamentally from that produced by injury to a peripheral nerve, or posterior nerve root. For, when a peripheral nerve has been divided, a considerable extent of the anæsthetic area remains sensitive to pressure, but insensitive to light touch. But, if the lesion lies within the spinal cord, tactile sensibility is diminished, or abolisbed as a whole, whether the touch be cansed by the lightest movement of hairs, by stimulation with cotton-wool, or by pressure. Occasionally, it is true, pressure produces the sensation of touch, when 
the less insistent forms of tactile stimulation fail to be appreciated. If an intramedullary lesion bas destroyed the impulses associated with the sensation of light touch, it will also interfere with those that unaerlie sensibility to pressure; that pressure can still be appreciated is due solely to the fact that it is a greater tactile stimulus, not to any difference in path between the impulses for light touch and for pressure.

In the previous section, we showed that the impulses for pain, heat and cold crossed the spinal cord to ascend on the opposite side. Here we sball consider the intramedullary course of tactile impulses.

The clinical records of cases of Brown-Séquard paralysis are more especially defective with regard to the condition of tactile sensibility; most of the stimuli that have been employed for touch are, in reality, tests for the recognition of pressure. Fortunately, however, the impulses, for light touch and pressure are combined into one tactile group during their passage up the spinal cord; pressure is only an extreme form of touch. We may, therefore, assume that if, in any case of Brown-Séquard paralysis, it is recorded that pressure was not appreciated, sensibility to light touch was also abolished.

Sensations of touch and pressure are wuch less frequently disturbed in cases of Brown-Séquard paralysis than those of pain, heat and cold. In the two cases observed with unusual care by Brown-Séquard himself, tactile sensibility was greatly diminished, or lost, over the same parts that were insensitive to pain, heat and cold, on the opposite side of the body to the disturbance of motion. Among 128 cases collected from the literature by Petrén, the anæsthesia to pressure reached the same development as that to pain, heat and cold in 39 only. In every instance the loss of sensibility to pressure was found on the same side as the analgesia.

This in no way proves that a unilateral lesion of the cord produces loss of tactile sensibility. It demonstrates only that, if this form of sensibility is abolished as the remote effect of an intramedullary lesion, the loss of sensa- 
tion will be found on the opposite side of the body to the motor disturbance.

We are able to add an excellent instance of this comparatively rare loss of tactile and pressure sensibility in a case of Brown-Séquard paralysis.

F. C. (Case 1), went to bed perfectly well on December 13, 1903. When he tried to get out of bed he found he "could not use his legs," and fell to the ground. On the afternoon of December 14 he found his left arm was weak. By Easter, 1904, his left arm and his left leg had recovered, and he returned to work. He had noticed little wrong with
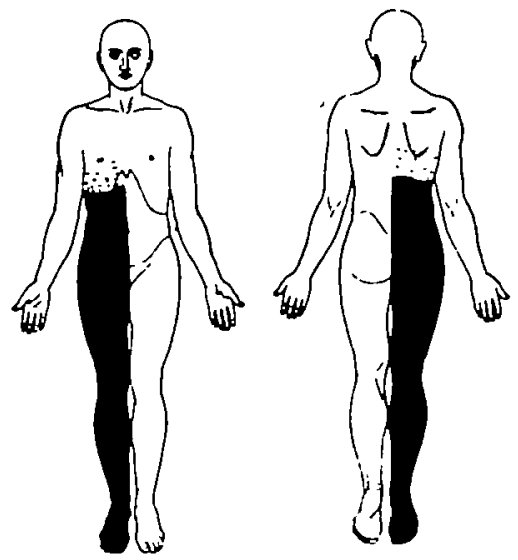

Fia. 18.

To show the loss of sensation to all forms of tactile, painful and thermal stimuli in Case 1. The froutal border was not sbarply defined, but merged gradually into parts of normal sensibility.

the other side, but within three weeks of the onset, during bis stay in the National Hospital, Queen Square, complete analgesia and thermo-anæsthesia had been discovered over the right half of the trunk and right leg.

$\mathrm{He}$ first came under our care in August, 1905, for an attack of acute rheunatism. During this time we discovered the condition of sensation, which has remained substantially unaltered up to the present time.

He now shows the following disturbance of sensation. All sensibility to cotton-wool is abolished over the right leg 
and right half of the trunk, as high as the level marked on Fig. 18. Within this area he is totally insensitive to pressure steadily applied; but, if the muscles are rolled from side to side during the act of pressure, he sometimes says, "You are rolling the front of my leg," or, "You are pressing me." This loss of sensation is associated with inability to distinguish the head of a pin from the point, or to appreciate difference in size.

All sensibility to pain is abolished over the same parts, whether the stimulus be a prick, an interrupted current or extreme pressure. Even a pressure of $20 \mathrm{kgms}$, applied to the outer aspect of the right knee, produces no pain, although, on a similar part of the left leg, the patient complains of pain with a pressure of $4.5 \mathrm{kgms}$.

The recognition of passive position is perfect in both legs from the toes to the hip.

All loss of motor power has now disappeared; his grasps are equal and there is no stiffness of either leg. The kneejerks are brisk but equal, and ankle clonus cannot be obtained. On neitber side does the plantar reflex give an extensor response.

Thus we conclude, that sensibility to touch and pressure is rarely disturbed in Brown-Séquard paralysis. But, if such a change exists,

(1) It is found on the opposite side of the body to the motor disturbance. (Case 1 and Case 4.)

(2) The impulses of light touch (cotton-wool, von Frey's hairs), deep touch and the tactile element of pressure are lost together. If the borders of the loss of sensation are ill defined, pressure can be appreciated over parts insensitive to stimulation with cotton-wool, in consequence of the greater intensity of pressure as a tactile stimulus. There is no evidence pointing to a separation of sensibility to light touch from that to the tactile aspect of pressure, such as is found in lesions of peripheral nerves.

\section{Section 3.-Sense of Passuve Position and Movement.}

Throughout this paper, in almost every section, our investigations have borne their tribute to the acumen and 
accuracy of Brown-Séquard. He stated that the impulses for the sensations of pain, heat and cold crossed to the opposite side of the cord; ultimately, the impulses for touch, though more rarely disturbed, followed a similar course. Not only our cases, but the whole of the work of the past, when anslysed, supports this view.

Much controversy has arisen regarding the further statement of Brown-Sequard, that appreciation of the position into which a limb has been passively placed is affected on the same side of the body as the disturbance of motion and reflexes. Nothing could be more convincing than a perusal of the besutifully recorded cases that came under his personal observation.

Among our observations, the following case (No. 2) is a striking corroboration of the view, that the impulses which underlie knowledge of the position into which a limb has been placed are interrapted on the same side as the motor disturbance. It is a peculiarly unequivocal instauce, because the patient was examined when all sense of passive position was perfect; yet the right leg was spastic, and sensibility to pain, heat and cold was abolished over the left half of the body and left leg. After the operation upon his spine, these signs remained unaltered, but be lost all power of recognising the position into which his right leg had been placed.

His history was as follows: On April 11, 1904, he was thrown out of a dog-cart on to his head. He became unconscious for a few seconds and, when be recovered his senses, found he was completely paralysed down the right side. From the moment of the accident he has been unable to appreciate pain, heat and cold applied to the left leg and left half of the trunk.

In May 1905, when first seen by oue of us, a little more than a year after this accident, he walked with a hemiplegic gait, swinging bis right hip, so that the toes of the right foot might clear the ground.

Over the left half of the body and the whole of the left leg below the level of the seventh cervical segment, he was inseusitive to all forms of pain, heat and cold. Light touch with cotton wool, deep touch and pressure were recognised 
everywhere, and were well localised. The sense of passive position was perfect in all four limbs, and he could recognise the position assumed by parts of the limb under electrical stimulation.

On the right side the hnee-jerk was greatly exaggerated, ankle-clonus was easily obtained and the plantar reflex gave an extensor response. The left knee-jerk was diminisbed and no clonus could be obtained in the left ankle; the plantar reflex on this side gave a flexor response.

Thus at this time the patient showed all the signs of that form of Brown-Séquard paralysis where motion is disturbed on the one side, sensibility to pain, heat and cold on the other, touch and pressure and the sense of passive position being entirely unaffected.

On June 25, 1905, Sir Victor Horsley exposed the laminæ of the cervical vertebræ and found that the third neural arch was fractured. All went well until a fortnight after the operation, when the patient noticed that the right side and right leg seemed stiff and cramped and he thought he had lost power.

But, when he was again seen by one of us, the only further change that could be discovered was a loss in the right arm and leg of the sense of position assumed passively. $\mathrm{He}$ could not touch his nose with his right forefinger if the eyes were closed. He could not tell into what position the thumb and fingers of the right hand had been placed, unless the movement was violently performed, and movements of the right wrist were badly appreciated. No movement of the toes of the right foot could be recognised.

At the same time, he was sensitive everywhere to stimulation by light touch and pressure, and the point touched was well localised. Thus, the operation had added loss of sense of passive position and movement on the right side to the loss of sensibility to pain, heat and cold, which previously existed over the left half of the body.

Case 16 also illustrates the loss of the sense of passive position and movement on the same side of the body as the disturbance of reflexes and of motion. R. A. H. suffered from a long-standing intramedullary lesion of the bulb and 
cervical region of the spinal cord, which had paralysed the left vocal cord and left half of the palate. The left kneejerk was exaggerated, clonus was obtained from the left ankle and the left plantar reflex gave an extensor response. On the right side, the reflexes were normal and no ankle clonus could be obtained.

Sensibility to all painful and tactile stimuli was disturbed over the whole of the left fore-quarter, but to heat and cold the loss of sensution occupied both apper extremities.

The legs remained normally sensitive to all forms of tactile, painful, and thermal stimulation, but the sense of passive position was profoundly disturbed in the left lower extremity, i.e., on the side of the alteration in motion and reflexes. Out of twenty movements, ten of which were dorsiflexion and ten plantar extension of the left ankle, he gave eleven false answers-

$$
\begin{array}{c|cc}
\text { Dorsiflexion } & 7 \mathrm{~W} . & 3 \mathrm{R} . \\
\hline \text { Plantar extension } & 4 \mathrm{~W} . & 6 \mathrm{R} .
\end{array}
$$

On the right side he made no mistake under similar conditions-

$$
\begin{array}{c|c}
\text { Doraiflexion } & 10 \mathrm{R} . \\
\hline \text { Plantar extension } & 10 \mathrm{R} .
\end{array}
$$

Sense of passive position was even more grossly affected in the toes of the left foot, but his answers were faultless and quick from those of the right foot.

Case 3 is another striking instance of a similar inability to recognise the position assumed passively by the toes; this loss was also found in the limb opposite to that insensitive to pain, heat and cold. This patient has been under observation for eight years, and we have therefore had ample opportunity of confirming our observations.

In March, 1898, she was suddenly seized with paralysis of the right leg accornpanied by loss of sensation to pain, to heat and to cold in the whole of the opposite limb. Since that time, her condition has remained materially unchanged.

When walking, she drags the right leg, which she holds stiftly, saying she dare not relax this leg, or she would fall. The whole leg is spastic, and voluntary movement is im- 
paired by its rigidity; but, in addition, there is some loss of power, especially in the movements of the right hip. There is no wasting, and the electrical response is everywhere normal.

Light touch and pressure are everywhere appreciated and accurately localised. Repeated examination failed to reveal any difference between her answers on the two sides. But the whole of the left leg and thigh below the level of the eleventh dorsal segment is absolutely insensitive to all forms of pain, heat and cold.

In this leg, every movement produced passively is quickly and easily recognised. She made no mistake when the left great toe was moved twenty times into s flexed or extended position. But on the right side, the side of the muscular rigidity and werkness, she failed repeatedly to recognise the position of the great toe. Here, our method of recording the results shows the difference between the condition of the two feet with remarkable distinctness.

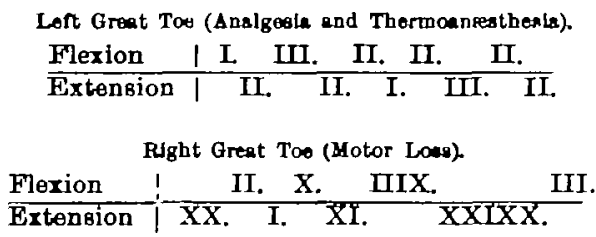

No difference could be observed between her power of appreciating movements of the ankles, knees or hips. Exactly similar results were obtained as far back as 1898 .

Here, again, the loss of the sense of a position assumed passively by part of the limb was found on the side of the motor disturbance, and not on the side insensitive to pain, heat and cold.

Thus, we conclude that, with regard to the impulses which underlie our power of recognising the passive position of a limb, Brown-Séquard's original statement was correct.

(1) The sense of passive position and movement is abolished on the same side as the motor disturbance, provided that the loss of motion is entirely unilateral and no single form of sensation is disturbed on both halves of the body. 
(2) The loss of the sense of passive position and movement is independent of the tactile impulses and of those which underlie the power of cutaneous localisation. But, as will be shown in the following sections, it is closely associated in Brown-Séquard paralysis with insbility to discriminate two compass-points.

\section{Section 4. -The Relation between the Power of Discriminating Two Points and the other Forms of Sensibility.}

In a previous chapter, we cited two cases to show that tactile sensibility might be perfect and yet the patient might be unable to discriminate two points applied simultaneously to the skin. Both of these cases revealed incidentally the close association between the loss of the sense of position assumed passively, and the power of discriminating two points.

Now, we have already ample evidence, that the power of recognising the passively assumed position of any limb tends to be disturbed on the same side as motion in Brown-Séquard paralysis. We should therefore expect that the compasstest would also show a diminished accuracy of discrimination on the sume side.

Bat it must be remembered, that the compass-test depends upon two entirely separate factors. In order that the test can be applied, the patient must be able to recognise that he is being touched. If sensibility to touch and pain is lost, the test is obviously inapplicable, because of the total absence of sensation, and not because of any necessary inability to discriminate two points. A diminution of tactile sensibility alone will affect the results of the compass-test, by decreasing the certainty with which the patient knows when he has been touched. This was the reason BrownSéquard stated that, in two of his cases, the compass-points were not normally discriminated over the non-paralysed limb. In both of these cases, tactile sensibility was profoundly affected on that half of the body opposite to the motor paralysis. (Cf. Case 4, p. 666.)

For the same reason Spearman [31], in his careful report, found that the compass-test gave bad results on both 
legs. We venture to suggest that on the right leg, which was analgesic and thermo-anøsthetic, this was mainly due to the grave defect in contact sensibility; on the left leg, which was spastic and paretic, the inability to recognise two points was associated with the loss of sense of position. The probability of this explanation is increased by the remarkable raising of the threshold for the compass-test which occurred on the left leg as the result of muscular fatigue, a condition scarcely noticeable in the right leg, where the cutaneous sensory loss was profound.

Since the compass-test depends upon two factors, which nay be separated in disease of the spinal cord, it is necessary to choose cases where one of these factors alone is demonstrably unaffected. It is obvious that a diminution of tactile sensibility will diminish the ease with which two points are discriminated; but we have other less elaborate tests for touch, and the true interest and value of the compass-test lies in the revelation of a loss of power of discriminating two points, although tactile sensibility is perfectly maintained.

Case 3 is an instance of such a condition. Over the left leg, sensibility to pain, heat and cold was abolished, whilst in the right foot the patient was anable to recognise the position into which her toes had been placed. To the finest tests all forms of touch were everywhere perfect.

When the compasses were applied longitudinally to the sole at a distance of $2.5 \mathrm{~cm}$., it was found that she was more accurate in her answers on the left foot (sensory loss) than on the right (motor loss).

$$
\begin{aligned}
& \text { Left } 2.5 \mathrm{~cm} \cdot \frac{1 / 9 \mathrm{R} .1 \mathrm{~W}}{2 \mid 10 \mathrm{R} .} \\
& \text { Right } 2.5 \mathrm{~cm} . \frac{1 \mid 8 \mathrm{R} .2 \mathrm{~W}}{2 \mid 8 \mathrm{R} .2 \mathrm{~W}} \text {. }
\end{aligned}
$$

For some hours before these tests, she had been resting in bed, and she was therefore tested again after walking about 150 metres without a stick. The result was striking. For whereas at $4 \mathrm{~cm}$. distance the test gave perfect results on the dorsum of the left foot, her answers failed grossly over a similar part of the right foot. 


$$
\begin{aligned}
& \text { Left } 4 \mathrm{~cm} \cdot \frac{1 \mid 10 \mathrm{R} .}{2110^{-}} \\
& \text {Right } 4 \mathrm{~cm} \cdot \frac{1 \mid 6 \mathrm{R} .4 \mathrm{w} .}{2 \mid 6 \mathrm{R} .4 \mathrm{~W} .}
\end{aligned}
$$

This brings out the considerable raising of the threshold for the compass-test which follows exertion, a fact first pointed out by Spearman [31].

On account of the stationary condition of her symptoms and her great quickness and trustworthiness, this patient forms a good instance of the close connection between delicacy in discriminating two points and \& recognition of the position of the limb.

When discassing disturbances of the sense of passive position and movement, we cited the case of R. A. H. (No. 16), where an intramedullary lesion of the bulb and cervical spinal cord had produced locally loss of sensibility in both upper extremities, whilst motion and the reflexes were disturbed on the left side only.

In this patient, the remote loss of sensation consisted of a profound distarbance of the sense of passive movement and position in the left foot and ankle. Together with this loss, it was found that his power of discriminating two points was profoundly affected over the left foot and leg. Thus, over the sole of the left foot his answers were bad when the points were separated to $8 \mathrm{~cm}$.

$$
8 \mathrm{~cm} \cdot \frac{1,10 \mathrm{R} .}{2 T 4 \mathrm{R} \cdot 6 \mathrm{~W}} \text {; }
$$

whilst over the right sole they were perfect at $4 \mathrm{~cm}$.

$$
4 \mathrm{~cm} \cdot \frac{1 \mid 10 \mathrm{R} .}{2 \mid 10 \mathrm{R} .}
$$

Over the outer side of the left leg he answered badly at $15 \mathrm{~cm}$.

$$
15 \mathrm{~cm} . \begin{array}{l|l|l|l}
1 & 6 \mathrm{R} . & 4 \mathrm{~W} \\
2 & 5 \mathrm{R} . & -5 \mathrm{~W}:
\end{array}
$$

although bis answers were perfect at $8 \mathrm{~cm}$. over a similar part of the right leg

$$
8 \mathrm{~cm} \cdot \frac{1 \mid 10 \mathrm{R}}{2 \mid 10 \mathrm{R}}
$$

VOL. $X X I X$. 
This difference in the sensibility of the two legs to the compass-test could not have been due to any alteration in tactile sensibility. For stimulation with cotton wool rwas appreciated equally when applied to both feet and to the outer side of both legs; over both soles, von Frey's hairs produced sensations of equal intensity. Stimulation with No. 8 and No. 5 was well appreciated; but stimulation with No. 4 applied to the soles could not be appreciated over either foot.

In conclusion, we believe that, if all forms of tactile sensibility are perfect in Brown-Séquard paralysis, (1) the power of discriminating two points will be found to be diminished over the side of the motor disturbance; (2) there is a close relation between loss of the sense of passive position and movement and the power of discriminating two points; loss of the one is usually associated with grave disturbance of the other.

\section{Chapter IV.}

Local Effects of an Intramedullary Lesion.

So far we bave neglected the local effects produced by a lesion of the spinal cord, and have dealt solely with its remote results on sensation. The skilled operator can now produce, with the help of rigid asepsis, an experimental division of one half of the cord, of such precision that the sensory effects at the level of the lesion, on the same side as the section, may be negligible. The sensory consequences of a stab in the back, producing a wound of the spinal cord, may also uuder favourable conditions be entirely remote. Even those remarkable cases of Browu-Séquard paralysis, arising suddenly and presumably due to spontaneous hæmorrhage, may show no permanent sensory changes at the level of the disease.

But, as soon as interference in the conducting paths is due to a growth or to a large hæmorrhage, such as not uncommonly follows fracture of the spine, the disease manifests itself by local disturbances which will form the theme 
of this chapter. The greater the extent of the lesion longitudinally in the spinal cord, the wider will be the area apon the surface of the body affected by these changes; and of all local lesions, the most widely extended is that of syringomyelia. At any rate in its early stages, this disease manifests itself by sensory changes on the same side of the body as the disturbance of motion; its signs and symptoms are essentially the local manifestations of a lesion of vast longitudinal extent.

In some cases of syringomyelia, the central growth reaches such proportions that long afferent paths are destroyed, and parts at a distance from the focus of disease become insensitive ; but, in essence, syringomyelia is a local disease, producing sensory changes at the level of the lesion.

\section{Section 1.-The Local Sensory Effects of an Intramedullary Lesion occur on the Same Side as the Disturbance of Motion.}

Every distarbance of cutaneous sensibility, due to the local effect of disease of the spinal cord, lies on the same side as the muscular wasting and loss of motor power, that is, on the side of the maximum lesion. This forms a fundamental difference between the local and remote effects of intramedullary disease.

This rule is well illustrated by the following case of gliosarcoma of the spinal cord (Case 9). In November, 1904, Joseph F. noticed a slight weakness of the left arm; soon afterwards, his neck became stiff, and be complained of tingling sensations in the fingers of the left hand. The weakness of the left arm gradually increased until February, 1905, when it became completely paralysed. During March and April, he became progressively weaker, and his legs began to give way.

In July, 1905, when admitted to the London Hospital, his left arm lay motionless on the bed by his side, completely paralysed for voluntary movement. The sinall mascles of the hand and, to a less degree, those of the forearm were wasted, but reacted in the normal manner to both the constant and interrupted currents. The grasp 
of the right hand was weak and its small mascles were somewhat wasted; all movements were well performed by the remaining muscles of the limb.

Sensation was profoundly disturbed over the whole of the left upper extremity. All sensibility to pain, to beat and to cold was lost over the areas shown in Figs. 19 and 20, extending as high behind as the occipital crest and occupying the whole of the arm, excepting only a small patch on its inner aspect.

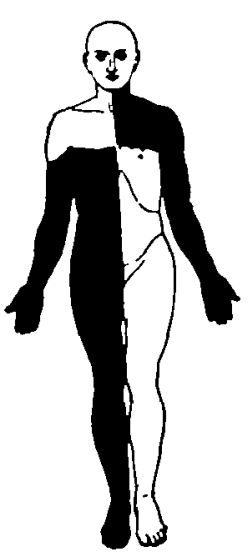

เพเ. 19.
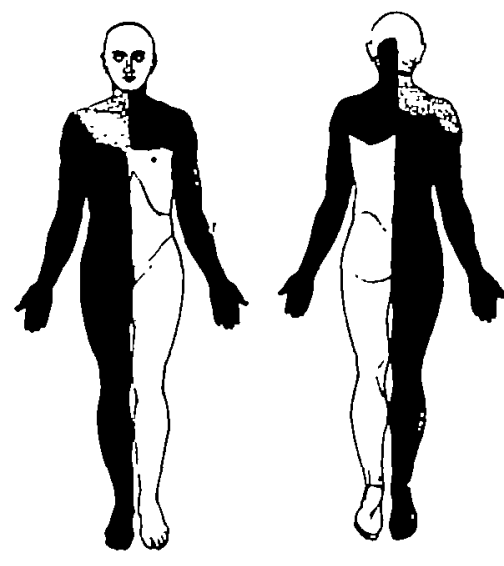

Fio. 2u.

To show the loss of sensation to penuful (fig. 19) and thermal (fig. 20) stimuli produced by a glio-sarcome of the spinal cord (Case 9). In the cervical region the growth destroyed mainly the left half of the cord. The condition in the left upper extremity represented the local, that on the right half of the body the remote effect of the lesion.

Light toaches and pressure were everywhere appreciated and well localised, but two points of the compasses could not be discriminated, even at a distance of $5 \mathrm{~cm}$., anywhere over the left hand. Over the right palm, his answers were perfect when they were $2 \mathrm{~cm}$. apart, and he made two mistakes only in twenty applications, when the points were separated by a distance of $1 \mathrm{~cm}$.

All sense of passive position and movement was lost in the left npper limb. When his eyes were closed, he was 
unable to indicate the position of his fingers in space or the direction in which they had been moved; and this was also the case with the wrist, the elbow and the shoulder-joint. The contrast in the condition of the two arms in this respect was striking; the patient recognised that he was entirely ignorant of the position of his left arm in space, whereas every movement of any part of the right arm was immediately recognised.

Such were the local effects produced by a tumour growing in the left balf of the spinal cord. The remote effects werè shown by profound sensory changes in the right half of the body, the right leg and right arm below the level of the fourth cervical segment. Within this area, he was insensitive to pain, to heat and to cold, but light touches and pressure were everywhere appreciated, and localised with accuracy. The sense of passive movement and position was disturbed in the left foot, that is, on the same side as the maximum lesion, but on the opposite side to the analgesia and thermo-anøsthesia.

This case shows, that a growth which destroyed the whole of the left half of the spinal cord produced complete analgesia and thermo-anæsthesia in the left arm at the level of the lesion. The remote loss of sensation to pain and temperature lay over the right or opposite half of the body. Moreover, the sense of passive position and movement was disturbed in the left or analgesic arm, in consequence of the local effect of the growth; whereas its remote effect was manifested by a loss of the sense of passive position and movement in the left foot. This was accompanied by total analgesia and therno-anæsthesia of the right half of the body, below the level of the fourth cervical segment Sensibility to touch and pressure remained unaltered until late in the disease. Finally, even this form of sensation was disturbed in the left arm.

Syringomyelia rarely disturbs all forms of sensation at the level of the lesion, but, as far 8 it occurs, the loss of sensation follows the rules that can be deduced from Case 10. So long as the symptoms remain unilateral, the loss of sensation will be found on the same side as the muscular 
wasting. This is so well recognised, that we need only mention shortly some cases among our collection which illustrated this rule.

In April, 1905, Walter J. H. (Case 12) was an excellent instance of syringomyelia with unilateral manifestations. Sensibility to pain and temperature was lost over the whole of the right forequarter, but no other form of sensation was disturbed. Neither hand was wasted and there was no loss of motor power. By Aagust, 1906, wasting and loss of power bad appeared in the right hand.

Similarly, Florence R. (Case 14), when first examined in 1899 , showed distinct wasting and loss of power of the left hand, accompanied by thermo-anæsthesia, confined to the preaxial half of the left forearm and band. Shortly afterwards, wasting of the right hand was noticed; but even as late as March, 1902, the loss of sensation was still confined to the left forearm and hand; but in November, 1902, part of the right hand also became insensitive to heat and cold. Here, again, the loss of sensation appeared on the same side as the loss of motor power and muscular wasting.

Such cases are examples of the rule that the local manifestations of intramedullary disease are found upon the same side as the disturbance of motion and the wasting of muscles.

Section 2.-The Nature of the Local Disturbances of Sensation produced by an Intramedullary Lesion.

In Chapter 3, we contrasted the nature of the loss of sensation due to destruction of peripheral nerves with that caused by a lesion of the spinal cord, choosing as an instance the phenomena of Brown-Séquard paralysis. From such a comparison, it became possible to formulate certain fundamental differences between the sensory loss in the two cases. We shall now attempt the more difficult task, and compare the characteristics of the loss of sensation, due to the local activity of an intramedallary lesion, with its remote sensory effects. The manner of response to each particular stimulas will be detailed in order, and we shall indicate the similarity and differences in the loss of sensetion produced by the two conditions. 
(a) Heat and Cold.

As is well known from the phenomena of syringomyelia, sensibility to pain, heat and cold may be lost without any other sensory defect. Any one of these forms of sensation may be disturbed separately, or to a varying amount, and it is a commonplace of neurology that thermo-anæsthesia may be the only sensory concomitant of this disease. Moreover, sensibility to heat is not infrequently more widely destroyed than that to cold. It is certain, therefore, that the local manifestations of an intramedullary lesion may disturb sensibility to heat, and leave sensibility to cold unaffected. C. $H$. (Case 11) was an instance of this condition; at one period of his illness, a large portion of his face was insensitive to heat though sensitive to stimulation with cold.

The converse condition may also be present, and the extent of the loss of sensibility to cold may exceed the loss to stimulation with heat.

But, ultimately, if the disease extends, the borders of the loss of sensation to heat will generally become coterminous with those of the loss to cold. The isolated anæsthesia to one form of temperature stimulation is of interest, solely as showing that a local lesion can disturb the one form of sensibility over parts that remain sensitive to temperatures at the opposite end of the scale. In this, the local manifestations of an intramedullary lesion agree with its remote effects described in Chapter III., and differ fundamentally from the loss of sensation caused by destruction of peripheral nerves.

With regard to the character of this loss of sensibility to beat, it is important to discover whether sensation is simultaneously disturbed for all temperatures we call warm or hot. This is frequently difficult to determine. For the borders of an area insensitive to thermal stimulation frequently merge gradually into parts of normal sensibility; here a temperature of $50^{\circ} \mathrm{C}$. will cause a sensation of heat, although the weaker stimulus of $40^{\circ} \mathrm{C}$. cannot produce an impulse of sufficient intensity to reach the sensorium. In the same way, temperatures of $22^{\circ} \mathrm{C}$. may produce no sensation, although coldness is easily evoked by stimula- 
tion with ice. Here the sensibility of the part to heat and to cold is lowered, and the intermediate degrees of temperature therefore become stimuli of insufficient intensity.

We have never been able to assure ourselves in any instance that, in consequence of a pure intramedullary lesion, the patient was sensitive to intermediate degrees of heat and cold but insensitive to extremes.

When the thermo-anæsthesia has remained stationary for a long period, so that this border of partial loss of sensation has disappeared, it is not difficult to show that sensibility to heat is destroyed as a whole. In a similar manner, it can be shown that over areas insensitive to cold, no temperature between ice and $24^{\circ} \mathrm{C}$. produces any sensation of coldness. In Case 14, careful experiments, made on September 10, 1906, and on many previous occasions, showed that sensibility to all degrees of heat and cold was destroyed over the left half of the face bounded by the middle line. Ice, water at $22^{\circ} \mathrm{C}$., at $38^{\circ} \mathrm{C}$., at $40^{\circ} \mathrm{C}$., and at $50^{\circ} \mathrm{C}$. were all called "a touch" over the left half of the face; whereas, when the middle line was passed, they were accurately discriminated and rightly named.

A similar condition was seen in W. J. H. (Case 12, Fig. 31) at the frontal border of the loss of sensation. On a photograph taken in April, 1905, this border corresponded exactly in shape and position with that marked out in September, 1906, showing that the loss of sensation had ceased to extend in this direction. At this border, no temperature at any degree between ice and $60^{\circ} \mathrm{C}$. produced any other sensation but that of touch. In this case, the abruptuess of the boundary between parts of normal and abnormal sensibility made it unusually easy to determine this fact.

But it is not only in syringowyelia that the local manifestations of an intramedullary lesion may produce a thermoanæsthesia, which conforms to this description. A rapidly advancing growth may cause a similar loss of sensation. Parts become insensitive to heat which are sensitive to cold, and no response may be evoked by stimulation with any degree of temperature which normally produces a sensation of heat. 'This was well shown by the case already 
cited on p. 587 (No. 9), where a tumour growing mainly in the left half of the spinal cord disturbed sensation in the left arm and right balf of the body. Here we had the opportunity of comparing the local thermo-anæstbesia on the side of the lesion with that on the opposite half of the body due to its remote manifestations; and in both cases, the character of the loss of sensation was the same. The left arm, equally with the right leg, was insensitive to all temperature stimuli.

To sum up, therefore: (1) the local action of a lesion within the spinal cord may produce a more extreme thermoanæsthesia to heat than to cold, or vice versâ. Parts sensitive to the one end of the scale may be insensitive to temperatures at the other end.

(2) We have found that an area insensitive to heat will also be insensitive to all temperatures that produce a warm sensation on the normal skin. 'lowards the edges of the thermo-anæsthetic areas, water at $40^{\circ} \mathrm{C}$. may produce no sensation, although $50^{\circ} \mathrm{C}$. is recognised as hot. This is due solely to the greater intensity of the stimulus of the higher temperature over parts of defective sensibility; it does not occur when the loss of sensation has remained stationary for long periods.

\section{(b) Pain.}

In every instance of Brown-Séquard paralysis where the patient was insensitive to cutaneous painful stimuli, he was also insensitive to the pain of deep pressure, although its gradual increase could be perfectly recognised. This characteristic association between cutaneous analgesia and loss of sensibility to the pain of deep pressure, is also found as one of the local manifestations of an intramedullary lesion.

Thomas B. (Case 13) is an excellent instance of this association. He was insensitive to the pain of a prick and of the interrupted current over both upper extremities and the upper half of the trunk (Fig. 6, p. 556). Over the whole of this area, pressure failed to cause pain, even when raised to $15 \mathrm{kgm}$.; and yet he was nowhere insensitive to deep) touch, and recognised the gradually increasing pressure. 
Elsewbere, outside the analgesic area, a pressure of from 3 to $5 \mathrm{kgm}$. invariably caused pain.

A similar condition was found in Case 12. Here the whole of the right upper extremity and the right half of the cervical and thoracic regions of the trunk were insensitive to the pain of a prick and the painful interrupted current (Figs. 21 and 22). Sensibility to touch, both superficial and deep, was everywhere perfect; but, over the whole of the right upper extremity, an increase of pressure up to $15 \mathrm{kgm}$. caused no pain. When we first saw him, the right upper extremity only was analgesic (Fig. 21). But
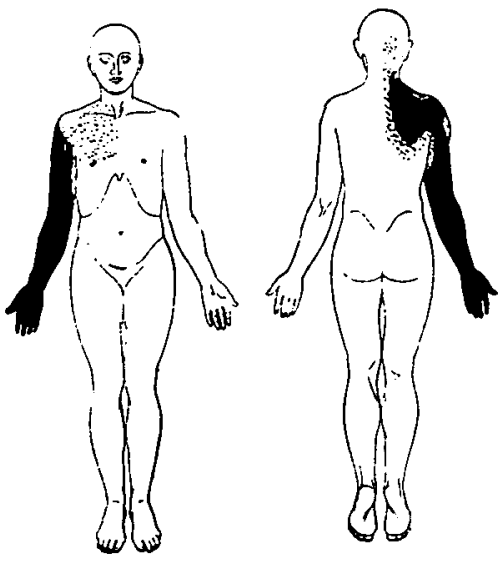

Fiti. 21
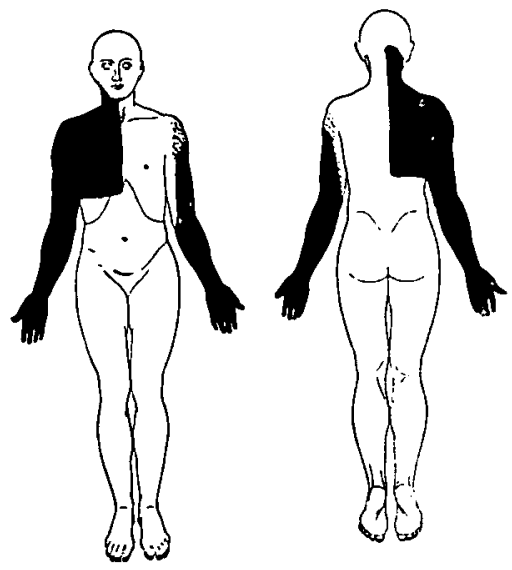

Fig. 22.

To show the loss of cutaneous sensibility to painful stimuli in Case 12. Fig. 21 shows the extent of the analgesia in 1905, fig. 22 the parts affected in 1906 .

later, the left hand and forearm also became insensitive to prick, and the loss of sensation is evidently advancing in this limb (Fig. 22). Here his behaviour when tested with the algometer was instructive. The loss of sensation to prick and to the interrupted current extended to the left elbow and at times even above that point. Over the palm, a pressure of $4.5 \mathrm{kgm}$. was painful, and he complained when the pressure over the flexor aspect of the foreaim reached $5 \mathrm{kgm}$. Although the forearm and hand were insensitive to prick and to the pain of an interrupted 
current, pain could still be produced by pressure little in excess of the normal.

Thus, over parts where the cutaneous analgesia has been recently acquired, increasing pressure not uncommonly produces a painful sensation. But, over an old-standing area of cutaneous analgesia produced by the local effects of an intramedullary lesion, pressure no longer produces pain ; and yet sensibility to the tactile element of pressure remains entirely unaffected.

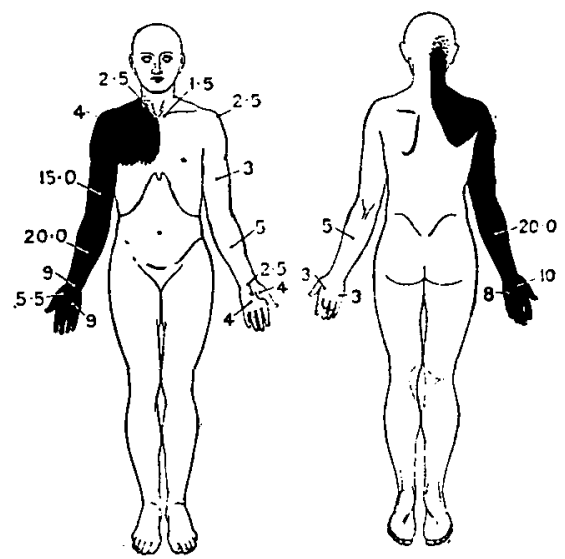

FIG. 23.

To show the loss of painful sensibility in Case 11 (Appendix, p. 698).

The area marked in black corresponds to the extent of the cutaneous analgesia. At various points are placed numbers corresponding to the pressure which caused pain. Thus 5.5 means that pain was caused by an ilgometer pressure of 5.5 kilograms. But such a number as $20=0$ means that no pain could be caused even by a pressure of 20 kilograms.

This persistence of sensibility to the pain of pressure over parts that correspond to the outworks of an advancing lesion, can lead to the following anomaly. The skin of the whole upper extremity may be insensitive to all painful stimuli, although pain can be produced by pressure over the palm of the hand. In Case 11, this phenomenon was the more striking in that the right arm and hand were entirely unaffected, and could be used for comparison with the analgesic extremity. On Fig 23, is shown the extent of the cutaneous analgesia, and at each point is given the 
pressure in $\mathrm{kgm}$. which produced pain. It is at once evident that the whole forearm, back and front, was insensitive to both cutaneous and deep pain. But, over the right palm, a pressure of $5.5 \mathrm{kgm}$. still produced pain; over a similar part of the normal palm, pain was evoked by a pressure of $4 \mathrm{kgm}$., a difference within the range of experimental error. Here the whole of the forearm on the extensor and flexor surfaces was insensitive both to painful cutaneons stimuli and to the pain of deep pressure; but, to the latter, the palm remained sensitive,

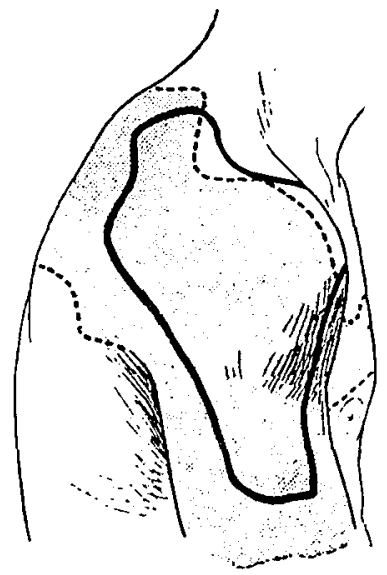

FIG. 24.

To show the relation of the deep hyperalgesia to the cutaneous analgesia in Case 11 (Appendix, p. 698). The area of cutaneous analgesia is coloured gray and euclosed by a broken line; the area hyperalgesic even to the lightest pressure is enclosed by a heavy black line.

though insensitive to cutaneous painful stimulation. This is evidently due to a difference in the segmental distribution of the afferent mechanism employed in the conduction of painful impulses produced by cutaneous and by deep stimulation. The pain produced by deep pressure on the palm is conducted by fibres which run with the muscular nerves. It probably enters posterior roots other than those which receive the afferent impulses from the skin of the same parts. If this were so, the impulses from the termination 
of the limb would probably enter those segments which correspond to the motor supply of the part. Now, the hand receives its motor innervation from the first thoracic and eighth cervical. In the case we have quoted, these segments, judging by the cutaneous loss of sensibility, would lie at the edge of the active lesion in the cord, and the hand would remain sensitive to the pain of deep touch, because it was represented by parts not in the focus of destruction.

This want of correspondence between the extent of the cutaneous and deep analgesia, produced by the local manifestations of an intramedullary lesion, led to the following extraordinary condition, in the case we have just considered (No. 11, Fig. 24). The skin of the whole upper extremity was analgesic to the prick of a pin and to the painful interrupted current. This man, however, suffered from a neuropathic destruction of the right shoulder-joint (Charcot's joint). This joint was excessively painful, and, over a considerable area enclosed within the thick black line on Fig. 22, he was profoundly hyperalgesic to even the lightest pressure; even pressure with the head of a pin caused him to cry out. At the time when this area of deep hyperalgesia could be marked out, the whole of the parts tinted gray on Fig. 24, were insensitive to all cutaneous painful stimuli. That is to say, there was a large area of the arm and shoulder abnormally tender to pressure, though the skin was entirely insensitive to the prick of a pin. Ultimately this hyperalgesia disappeared with the gradual advance of the loss of sensation. (For further details, vide Appendix, p. 702.)

In conclusion, we find that the analgesia, produced by the local manifestations of an intraspiual lesion, consists essentially of insensibility to the pain of both cutaneous and deep stimulation. This loss of sensation to painful pressure exists without any disturbance of sensibility to its tactile element; the patient not only knows he is pressed, but recognises the gradually increasing pressure.

In these characters, the analgesia produced by the local manifestations of intramedullary disease corresponds exactly with that due to its remote effects. 


\section{(c) Touch and Pressure.}

One of the most striking differences between the form assumed by the loss of sensation in Brown-Séquard paralysis and that which follows injury to peripheral nerves, lies in the behaviour of the patient to the stimuli of superficial and deep touch. If sensibility to touch is disturbed in BrownSéquard paralysis, the patient no Ionger responds to either form of stimulation.

Loss of tactile sensibility is a rare local manifestation of syringomyelia; but we are able to bring forward two

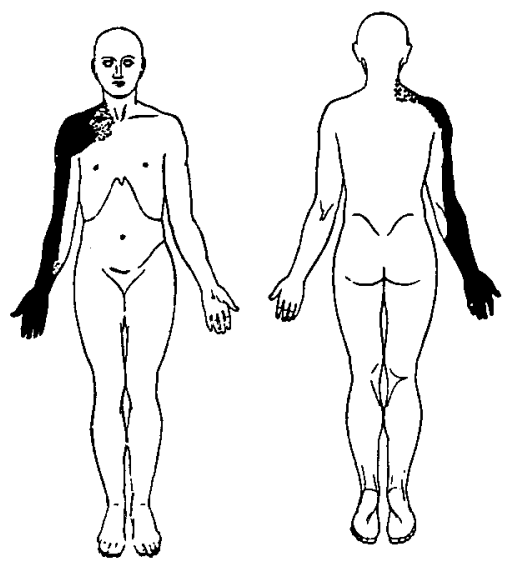

FiG. 25.

To show the loss of tactile sensibility in George B. (Case 15, Appendix, p. 720). Over the area marked black he was insensitive to all forms of tactile stimuli, superficial and deep; over the dotted area he was insensitive to light touch, but sensibility to deep touch, though present, was certairly somewhat diminished.

instances from our collection. From these cases, it would seem that the form assumed by the loss of tactile sensibility corresponds exactly with that of the remote tactile anæsthesia in Brown-Séquard paralysis. Sensibility to light touch and sensibility to pressure were lost over almost exactly the same extent.

Fig. 25 shows the area insensitive to light touch and to pressure in the case of George B. (No. 15). For seven 
years he had lost sensation in the right hand, accompanied by a gradually increasing inability to write. In October, 1906, the sensations of pain, heat and cold were lost over the right upper extremity and the greater part of the right half of the bead and neck. Sensibility to pressure was destroyed over the area marked in black on Fig. 25 ; over this area, together with the dotted parts, he was entirely insensitive to light touch. These two areas corresponded closely, except at the extreme frontal border, where the extent of the loss of sensation to light touch somewhat exceeded that of the loss to pressure. But, since stimulation with No. 8 of von Frey's hairs no longer produced any sensation when the border of loss to light touch was passed, we may assume that deep sensibility was also diminished. Pressure caused a sensation only by its greater intensity as a tactile stimulus. On the inner aspect of the arm, the borders of the anæsthesia to the two forms of tactile stimulation coincided almost exactly.

The second case where tactile sensibility was disturbed as a local manifestation of intramedullary disease, is that of R. A. H. (No. 16, Appendix, p. 727). When this man was first seen in March, 1904, all loss of seusation was confined to the left arm and neck, except that to cold, which was bilateral. Tactile sensibility was completely abolished over the whole of the left upper extremity. 'The motor disturbance was represented by paralysis of the left vocal cord and the left half of the palate. 'T'be left knee jerk was greatly increased, clonus was obtained from the ankle and, on the same side, the plantar retlex gave an extensol: response.

Thus it would seem that, in those rare instances where syringomyelia produces loss of sensation to touch, it also causes corresponding anæsthesia to the tactile element of pressure.

More rapid growths, starting within the spinal cord, frequently spread to the posterior roots, or produce secondary metastases which become extramedullary. 'I'bis makes the results obtained from such malignant growths extremely unsatisfactory. Many of the sensory phenomena 
they cause are of mixed origin, and not due solely to intramedullary disease.

As far as our material goes, we believe that, when tactile sensibility is disturbed at the level of the lesion in consequence of intramedullary disease, the part becomes insensitive to stimulation with touch, both superficial and deep. Towards the extreme edges, pressure may be appreciated, but the shin may be insensitive to the lighter stimulus; this is due solely to difference in the intensity of the stimulus, and accurate tests will show that, even over this borderland, sensibility to deep touch is diminished.

Thus, the condition of tactile sensibility at the level of the lesion closely resembles that due to its remote effects.

\section{(d) Passive Movement and the Discrimination of Two Points.}

Among the distant manifestations of an intramedullary lesion, any loss in the sense of passive position and movement was always found to be associated with defective answers to the compass-test. (Chapter III., Section 3, 4.) A similar relation is found, when this form of sensation is disturbed at the level of the lesion. So long as the disease remains purely intramedullary, there is not that close association between the discrimination of two points and sensibility to light touch usually found after lesions of peripheral nerves or posterior roots; nor is the power of recognising passive movement and position associated with the integrity of deep touch.

Of this we can bring forward one unusually perfect example. Joseph F. (Case 9) suffered from a glio-sarcoma, which destroyed the spinal cord in the cervical region more extensively on the left side than the right. He was unable to recognise the position of any part of the left upper extremity, and no passive movement of the joints, however extensive, produced any sensation. And yet, at first, sensibility to both superficial and deep touch was unaffected.

Over the left band, he was unable to discriminate two points of the compasses when separated for a distance of $5 \mathrm{~cm}$. ; over the right hand he made two false answers only at $1 \mathrm{~cm}$., and at $2 \mathrm{~cm}$. he was invariably correct. 


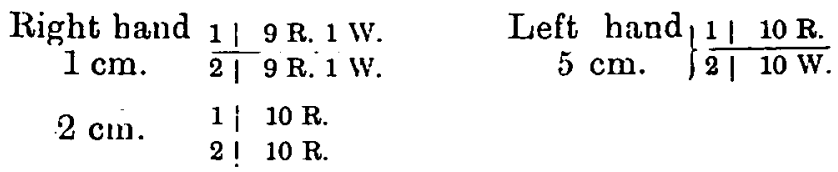

Here the power of discriminating two points was profoundly impaired, although tactile sensibility was at that time unaffected, and the sense of passive movement and position was lost, in spite of the retention of sensibility to pressure.

Thus, it would seem that, when the loss of sensation at the level of an intramedullary lesion includes disturbance of the sense of passive position and movement, the discrimination of two points is also affected.

\section{(e) Sumnary.}

A survey of the character assumed by the loss of sensation at the level of a pure intramedullary lesion of the spinal cord has led to the conclusion, that this loss follows the central rather than the peripheral type, although it is situated in those parts which lie on the sane side as the disease. If sensibility to heat is disturbed, the patient no longer recognises temperatures between $30^{\circ} \mathrm{C}$. and $60^{\circ} \mathrm{C}$. But, over the same parts, he may be sensitive to all temperatures which normally produce a sensation of cold. Where the skin is insensitive to painful stimuli, deep pressure will no longer be painful. Sensations of light touch and the tactile aspect of pressure are linked together, so that the former is a less, and the latter a more intense, degree of tactile stimulation. When the patient becomes unable to recognise the position of his linub, assumed passively, it will be found that he is also unable to discriminate two points at distances much in excess of that normally requisite.

\section{Chapter V.}

Afferent Segmentation within the Spinal Cord.

Whenever several observers, working on the same problem, bring out results which differ greatly, in spite of fundamental similarity, it is certain they have adopted 
different methods. The present state of text-book knowledge on segmentation of the central nervous system is an instance of the confusion which may arise from an attempt to combine the results of different observers, without recognising the significance of the methods by which they have been obtained. (Seiffer [27], Lewandowsky [17], Oppenheim [20]).

Those who have worked on the segmentation of the afferent nervous system from their own observations, and by original methods, can be classified into the following groups.

(A) Distribution of the Posterior Roots.

(1) Sherrington [30] determined the total afferent distribution of each posterior root, by the method of residual sensibility. By dividing a number of posterior roots above and below the one nerve root under examination, he left a sensitive area surrounded on all sides by anæstbesia. This sensitive area must have been innervated by the one remaining posterior root. But, of the specific function of these afferent nerve fibres, he remained of necessity ignorant. His areas correspond to the maximal extent of the fibres of one posterior root, whether they be those of epicritic, of protopathic or of deep sensibility. We shall therefore speak of the areas worked out by Sherrington as maximal root areas.

(2) Head and Campbell [12] recorded the areas on the surface of the body mapped out by the eruption of herpes zoster. They then determined in seventeen instances by post-mortem examination which posterior root ganglion was the seat of the disease. Now, it is obvious that, if the whole of one ganglion is affected, the extent of the eruption will correspond to the distribution of those fibres only which, when irritated, can cause vaso-dilatation and destructive lesions on the skin. Head and Sherren [13] have shown reason to believe that such fibres are closely associated with the protopathic system. (Cf. also Bayliss [1]).

It is therefore probable, that the areas marked out by herpes zoster correspond to the segmiental arrangement of 
the protopathic fibres as they pass through the posterior root ganglion.

\section{(B) Segmentation within the Spinal Cord.}

(1) As revealed by irritation (Hyperalgesia).

Head [11] worked out a scheme of intramedullary segmentation based on the hyperalgesic zones which arise in consequence of visceral irritation. Disease of an internal organ produces impulses which pass into the spinal cord by the white rami. The segment upon which they impinge is excited, and pain is produced. But, at the same time, all potentially painful impulses passing into this segment from the somatic afferent nerves are exaggerated, and ultimately the body wall may become tender.

These hyperalgesic zones represent the extreme projection on the surface of the body of those segments within the spinal cord which receive painful impulses from the periphery.

(2) As revealed by loss of sensation due to intramedullary lesions (Kocher [15], Allen Starr [33 and 34], Thorburn [35 and 36], Wichmann [40]).

Of these observers, Kocher alone faced the difficulty and determined to record the upper level of the analgesia in a large number of cases of verified spinal lesions. That part of Wichmann's valuable monograph which deals with the spinal cord is also based on the loss of sensation, produced by intramedullary disease; but, in his general scheme, he attempts to combine the results obtained by other means. Thorburn assumed that the areas of loss of sensation corresponded to the form and extent of the supply of segments within the spinal cord, and failed to appreciate that they represented those parts only, which were not supplied from the lowest intact segment. Moreover, be failed to differentiate between the loss of sensation due to destruction of roots as they enter the spinal cord and that of intramedullary segments.

A want of appreciation of the different methods employed by these observers, and the non-recognition of the fact that 
their results applied to different levels of the nervous system, have led to endless confusion.

The appearance of Sherrington's work on the posterior roots caused clinicians to revise their views with regard to the distribution of sensory disturbances in certain wellknown diseases such as tabes dorsalis. Max Laehr [16] was the first to recognise that the loss of sensation in syringomyelia was distributed segmentally, at a time when most observers believed that its arrangement more nearly corresponded to that found in hysteria. Since then, many clinicians have acknowledged the truth of his observations and have amplified his statement in various directions.

Clinical observers have relied on the extent of the loss of sensation in their attempts to prove the segmental distribution of a lesion within the spinal cord. All would have been well, had they correlated their results solely with the borders of the loss of sensation in verified cases of interruption of the cord at various levels. But the attempt to compare these areas of loss of sensation from intramedullary disease with the maximum supply of posterior roots led to nothing but confusion.

No clinician seems to have recognised, that the complete cutaneous distribution of a nerve, of a posterior root or of a segment of the spinal cord, could only be discovered by observing how much of the skin was sensitive, when that afferent path alone remained intact. If we wish to know how much of the palm is supplied by the median nerve, we cannot do so by observing the extent of the loss of sensation caused by its division. A case must be selected where the ulnar, the radial and the external cutaneous nerves have been divided; then, any part of the hand which remains sensitive must receive its innervation from the median. ( $C f$. Head and Sherren [13].)

This is the method of residual sensibility invented by Sherrington, and so ably employed by him to discover the maximum afferent distribution of each posterior root. 
If we wish to determine the exact level of the affected intramedullary segment, we are compelled to adopt an indirect method. For lesions witbin the spinal cord, such as those produced by fracture of the spine or rapidly spreading growths, lead to wide-spread destruction. However: well defined may have been the disturbance of sensation, some disease will be found corresponding to parts beyond its limits. Moreover, the opportunities are too limited for correlating these areas of residual sensibility with the extent of intramedullary disease.

Head [11] showed that there is a close correspondence between the distribution of the tenderness caused by irritation of the segments within the spinal cord, concerned with the reception of painful impulses, and the areas marked out on the skin by the eruption of herpes zoster. Now, herpes zoster is due, in most cases, to acute inflammation of a posterior root ganglion, whereas the tender areas in visceral disease are due to the irritation of intramedullary segments. But, though all the cells and the fibres peculiar to them must be affected by the profound inflammation of the ganglion, one system only, as far as we know, can produce an antidromal effect upon the skin. These are the fibres shown by Bayliss [1] to have their cells of origin in the ganglion of the posterior root. Bayliss, in conjunction with one of us, has been able to show, that these antidromal fibres are capable of excitation in the divided nerve of a cat, five weeks after it has been reunited to the central nervous system. In the time and manner of their regeneration, these fibres closely resemble those of the protopathic system. Thus, it is probable that this power of producing acute changes in the skin of the periphery is a function of fibres, which run in the protopathic system.

On this bypothesis, the eruption of herpes zoster would correspond to the distribution of the fibres and cells of the protopathic system in the ganglion of the posterior root.

Now Head and Sherren [13] showed, that division of several posterior nerve roots caused a more extensive loss of protopathic than of epicritic sensibility. Expressed 
crudely, sensibility to painful cutaneous stimuli was lost over a larger area than sensibility to light touch. From this fact alone it is certain, that the protopathic fibres in the posterior roots supply areas which overlap one another to a less extent than those of the epicritic system.

It is therefore not surprising to find a comparatively close correspondence between the form and extent of the areas marked out by herpes zoster, and the hyperalgesic zones of visceral disease. They belong, it is true, to different levels of the nervous system; the herpetic zones correspond to the distribution of those fibres which conduct all the cutaneous painful impulses, whilst the others represent the segments of the spinal cord into which those impulses impinge.

Since it is impossible to obtain enough material to determine the enumeration of the segments by means of destructive lesions of the spinal cord, it is justifiable to turn to the known levels in herpes zoster. For, since the seventeen post-mortem examinations by Head and Campbell, it has become possible to determine the site of the lesion with precision, in most cases of this disease. Even should it ultimately turn out that the likeness between the areas of tenderness in visceral disease and those of herpes zoster bas been exaggerated, we can still determine the numerical level of an intramednllary segment, by comparing the extent of the residual sensibility with the areas marked out by herpes zoster.

(a) The Sensory Disturbance at the Level of the Lesion shows signs of Intramedullary Segmentation.

By applying this method of residual sensibility to cases where the sensory impulses have been interrupted by disease within the spinal cord, it would be possible to arrive at a notion of the caudal borders of the intramedullary segments for touch, for pain, for heat and for cold. In every case, the border must be determined at which sensation to each of these stimuli ceases.

But, unfortunately, in an overwhelming majority of cases, the boundary between parts of normal and abnormal sensibility is ill defined. The upper border of the anæs- 
thesia for any one stimulus may differ in position according to the method of testing. If, for instance, we start from parts of normal sensibjlity, the patient's attention is awakened, and he will respond to the stimulus even within areas which would appear to be insensitive if the testing began in the reverse direction. The border obtained by passing from normal to abnormal parts may differ greatly from that at which sensation is present, when we travel in the reverse direction. If so, it is certain that the specific
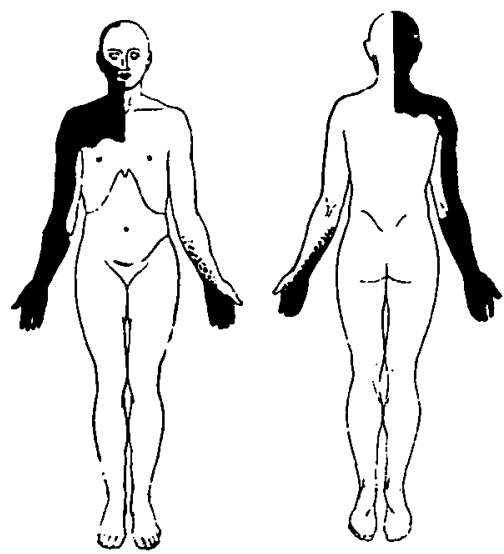

Fic. 26.

To show the loss of sensrtion to cutaneous paiuful stimuli in Case 15. (Appendix, p. 720.)

Note the area of resicual sensibility on the inner aspect of the right arm; its frontal border corresponds to that of the $2 \mathrm{nd}$ thoracic segment.

sensation we rse testing is diminished, but not absolutely lost, over an area which extends between the two borders. Such cases are useless for determining the form and position of intranedullary segmental borders. ${ }^{1}$

In order that an area of disturbed sensibility can be used to investigate intramedullary segmentation, the position of the border of residual sensibility must remain constant from day to day. Moreover, the border at which the specific

' For an instance of such a border, cf. Case 6, p. 674. 
sensibility ceases must correspond closely with that at which it begins when testing in the opposite direction.

Unfortunately, such well-defined areas of total loss of sensation are rare manifestations of intramedullary disease. But they occur sufficiently frequently for us to be certain that the boundary between sensitive and inseusitive parts corresponds to the border of an intramedullary segment. For instance, in Case 15, an area on the inner aspect of the right arm alone remained sensitive to cutaneous painful stimuli (Fig. 26). The border of this residual sensibility should correspond to an inter-segmental boundary.
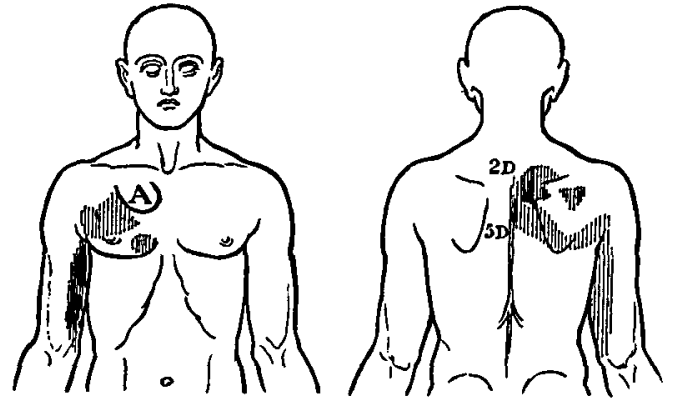

Fig. 27.

To show the area cf superficial tenderpess due to an aneurysm of the aorta. The frontal border of this area correspon.is to that of the 2 nd thoracic segment.

Now, the only scheme of intramedu'lary segmentation based on any method except loss of sensarion is that of the hyperalgesic zones in visceral disease. Compare the area of residual sensibility on Fig. 26 with the tendernass found in a case of aortic disease (Fig. 27). It will be seen how closely the extent of the one corresponds with that of the other.

From a case of herpes zoster examined after death (Head and Campbell, Case 7), we know that this area of residual sensibility must have corresponded to the distribution of the second thoracic segment (Fig. 28).

Another boundary, which remained over a considerable 

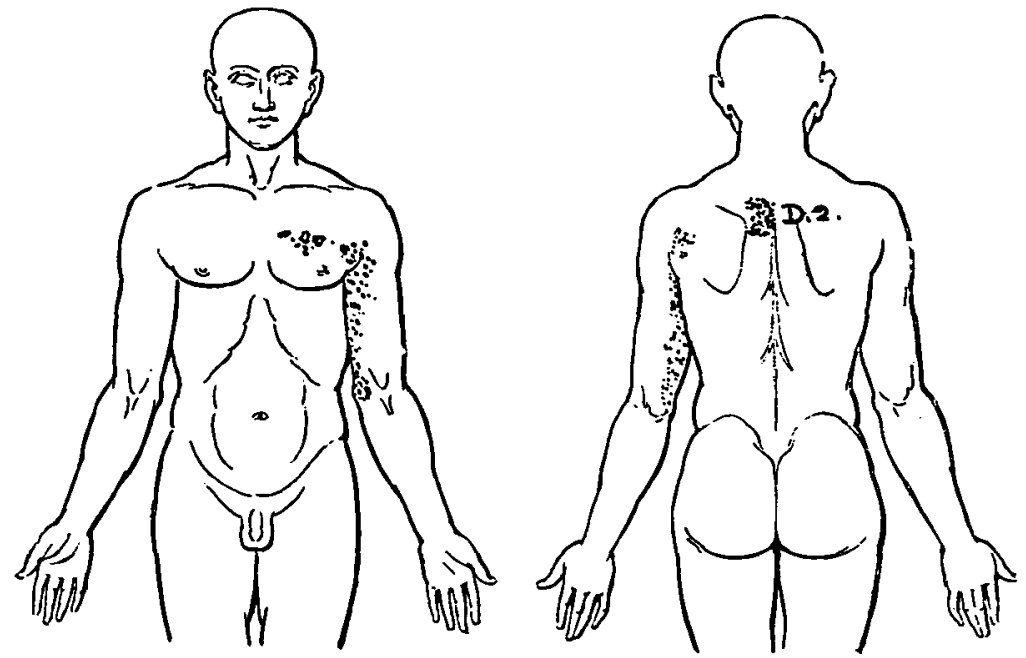

FIG. 28.

To show the distribution of the eruption in a case of herpes zoster, proved by post-mortem examination to be due to inflammation of the ganglion of the 2nd thoracic posterior root. (Head and Campbell [12], Case 7.)
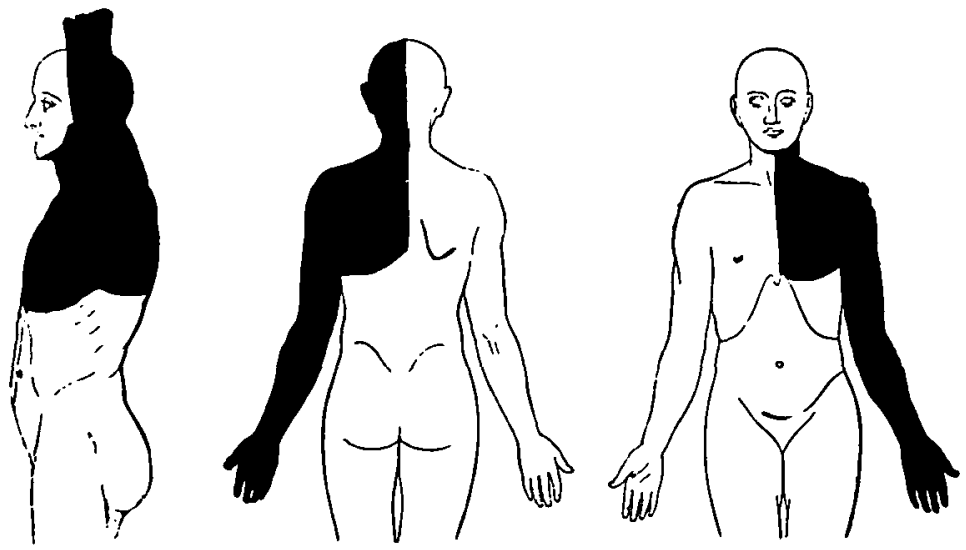

Fig. 29.

To show a well-defined boundary between parts insensitive to prick and those of normal sensibility. (Case 16, Appendix, p. 727.)

The boundary of the residual sensibility corresponds to the frontal border of the $7 \mathrm{th}$ thoracic segment. 
period constant to cutaneous painful stimuli, is shown on Fig. 29. This was taken from a case of syringomyelia (Case 16), where the disease affected mainly the medulla and cervical spinal cord.

Compare this intramedullary segmental area with the distribution of herpes zoster in a case where the ganglion of the seventh thoracic posterior root was diseased (Fig. 30). It will be seen how closely the frontal border of this eruption corresponds to the boundary of residual sensibility to painful stimulation in Fig. 29.
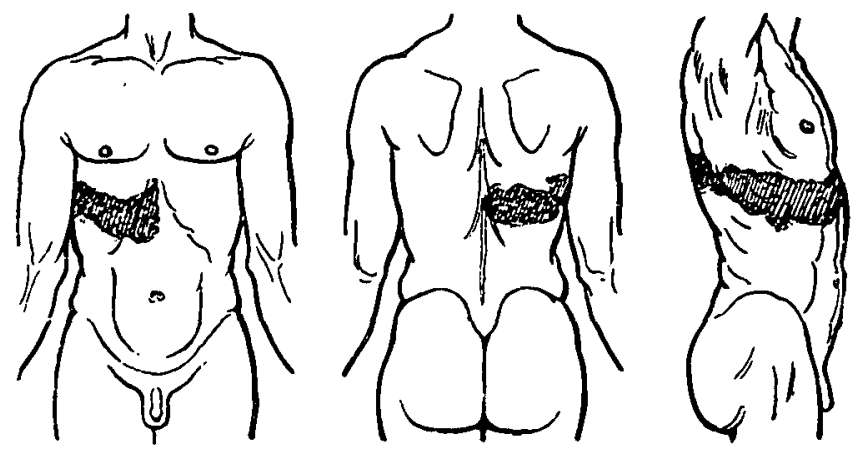

Fig. 30.

'To show the distribution of the eruption in a case of herpes zoster proved by post-mortem examination to have been due to inflammation of theganglion of the 7 th posterior root. (Head and Campbell, Case 2.)

We have compared the border of an area of residual sensibility with the border of a zone of hyperalgesia, due to the disturbance of an intramedullary segment by visceral irritation. But a glance at Figs. 26, 29 will show that the segmental nature of the disturbance in the two instances of syringomyelia could have been deduced even from an examination of the extent of the analgesia, for the boundaries of the area insensitive to painful stimulation corresponded closely with that of the parts sensitive to the same stimuli. Thus, neighbouring segmental zones cannot overlap one another extensively on the trunk. The amount to which the distribution of one segment encroaches 
on that of its neighbours is probably less than one half its own extent. Thus it comes about that even the loss of sensation to prick, to heat and to cold may closely represent the true segmental distribution of the intramedullary centres.

The frontal border of the loss of sensibility to pain, produced by cutting off all these segments below a certain level, will cause a loss of sensation, that is only slightly lower than the caudal border of the same segment shown by residual sensibility.

For this reason, those observers who have fixed their attention on the cutaneous analgesia and thermo-anæsthesia produced by lesions of several segments of the cord have come closer to the true segmental distribution than would have been the case, had their contention been correct that these segments overlapped greatly.

But this is true only for the loss of sensation to cutaneous painful and thermal stimuli. An intranedullary lesion may manifest itself locally by an area of cutaneous analgesia and thermo-anæsthesia whose borders correspond almost exactly to those at which sensation to pain, heat and cold ceases. But much of this area may be sensitive to deep painful stimuli, such as excessive pressure.

Moreover, tactile sensibility is usually disturbed over a considerably smaller extent, and the borders of this loss of sensation do not correspond to those of the cutaneous analgesia.

It is therefore of fundamental importance, in every case, to test each form of sensation separately, and to record the border at which it ceases and the border at which it begins, when the stimulus travels from sensitive to insensitive areas. If these two borders correspond closely and if the same borders are obtained on several different occasions, it is possible to say that this area may be a segmental border. The form and extent of the boundary of the residual sensibility only will determine if the disturbance of sensation is the expression of intramedullary segmentation.

We bave brought sufficient evidence to prove the truth of Max Laehr's [16] original statement, that the phenomena of loss of sensation, caused by intramedullary disease, pointed 
to a segmental arrangement of the afferent mechanism at the level of the lesion. But it will be well in the light of this knowledge to examine some of those remarkable borders on the face between parts of normal and abnormal sensibility, so commonly found in cases of syringomyelia.

We shall bring forward such cases only; where these borders remained unaltered for considerable periods. For, when the extent of the loss of sensation is spreading, a single examination may yield results which are transitory, due to the want of adjustment of the higher sensory mechanism to shifting structural changes. But, when a sensory border has remained constant over a long period,

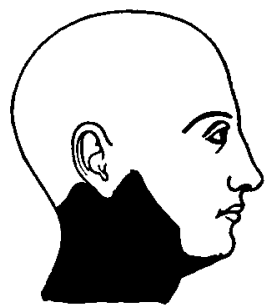

FIG. 31.

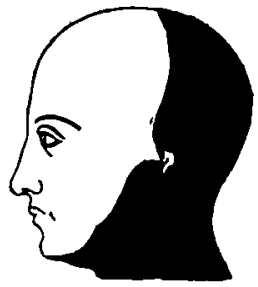

FIG. 32.

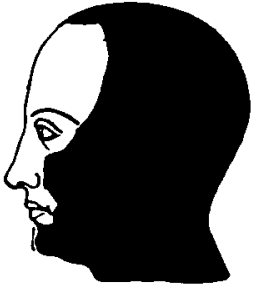

FIG. 33.

Fig. 31.-From Case 12 (Appendix, p. 705). To show the extent of the residual sensibility to painful and thermal stimulation in 1906.

Fig. 32.-From Case 16 (Appendix, p. 727). To show the extent of the residual sensibility to painful cutaneous stimuli.

Fig. 33.-From Case 14 (Appendix, p. 714). To show the extent of the residual sensibility to painful cutaneous stimuli, taken from a series of observations made between November, 1903, and February, 1905.

as occurs not infrequently in cases of syringomyelia, we can assume that it represents the division between those parts of the central nervous system still capable of function and those in which this power has been destroyed.

The disturbances of sensibility to heat and to cold follow the same segmental arrangement as those for pain. In cases of syringomyelia, it is sometimes possible to find one segmental border marked out by the sensory disturbance to heat, another to cold and a third to pain. Such borders are not, however, usually fixed, and do not therefore come within the subject of this section. 
In Case 12, the area insensitive to heat and cold, when passing from normal to abnormal parts, was bounded above by the line on Fig. 31. This was no transitory border, for it has persisted from April, 1905, until October, 1906.

A similar border on the face, associated with a completely different loss of sensibility on the scalp, is seen in Fig. 32. Here the loss of sensibility to cold extended almost as far forwards as the frontal border of the hairy scalp.

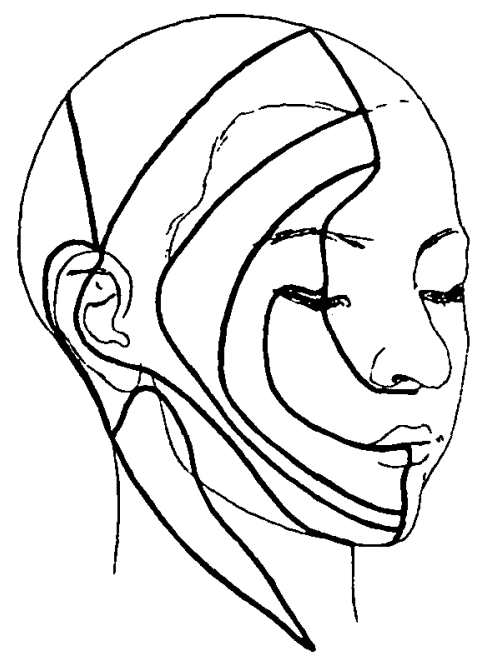

Fig. 34 .

Taken from Schlesinger [25] to show the method by which, according to this author, the loss of sensibility advances in lesions of the upper part of the spinal cord and medulla oblongata. Note how closely these borders correspond to those of the tenderness in visceral disease of the organs of the head and face.

Another remarkable area is shown on Fig. 33, taken from Case 14. This was worked out by each of us independently on many separate occasions, between November, 1903, and February, 1905. At this border, all sensibility to cutaneous painful stimuli was lost, and the area over which sensation was present to these stimuli was sbarply defined.

We have already shown that on the trunk the residual sensibility to pain, heat and cold tends to follow the dis- 
tribution of intramedullary segments. It is therefore probable, that these areas on the face are also distributed segmentally. They certainly do not correspond to the peripheral divisions of the trigeminal nerve.

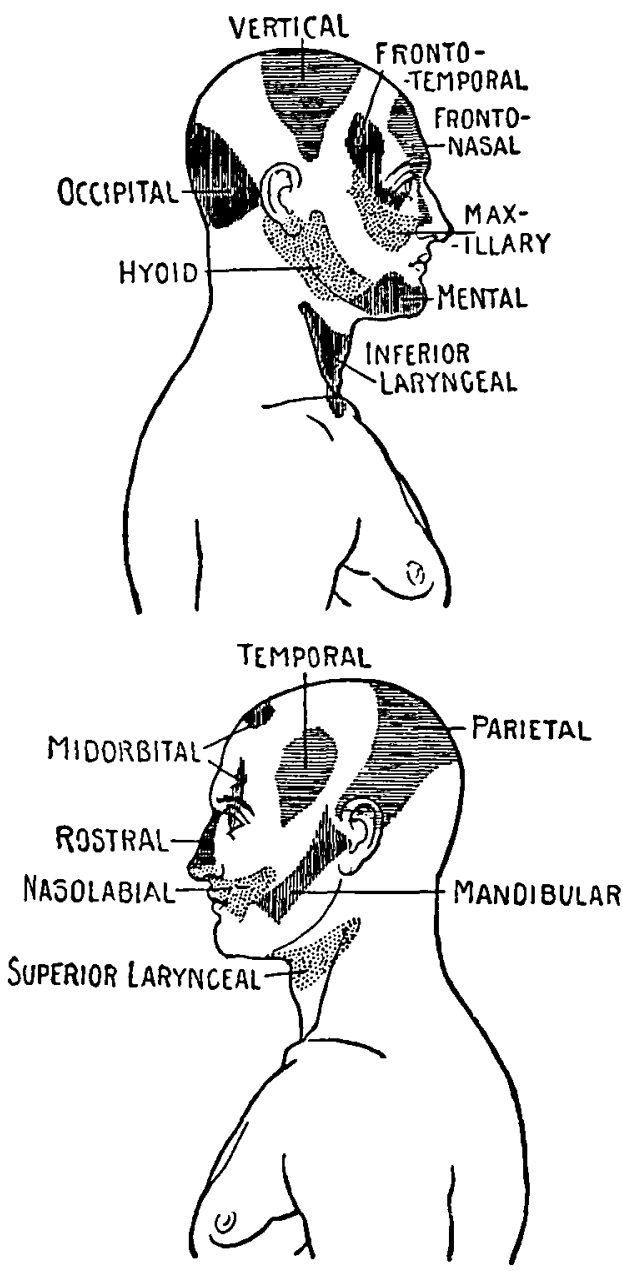

Fig. 35.

To show the extent and distribution of the tender areas which may accompany reflected visceral pain due to disease of the organs of the head and neck. (Taken from Head 11.)

The line on Fig. 31 can scarcely represent the posterior border of residual sensibility of the third division of this 
nerve, and it certainly does not correspond to that of its second division. On Figs. 32 and 33, the areas of residual sensibility are inexplicable from the point of view of the peripheral branches of the trigeminal.

The clue to the significance of these areas of residual sensibility is given by the tenderness which accompanies disease of the organs of the head and neck. Fortunately, most of these areas upon the face can be worked out from diseases of the teeth; and, as one tooth only is not infrequently diseased in patients otherwise in perfect health, single areas make their appearance on the face more often than in any other part of the body.

Compare the diagrammatic representation of these areas of tenderness on Fig. 35 with the parts that remained sensitive to pain, to heat or to cold on Figs. 31, 32, 33. It will be obvious, that here lies the key to the otherwise inexplicable distribution of the sensory disturbances of the head and neck, produced by a local intramedullary lesion.

Fig. 31 shows that all segments anterior to the third cervical and hyoid areas were unaffected; but in Fig. 32, the vertical parietal and occipital areas were added to those insensitive in the previous case.

On Fig. 33, it is evident that the only sensitive parts remaining corresponded to the segments in front of the vertical, the temporal and maxillary areas.

Thus, in spite of the difficulties which surround the determination of these tender areas accompanying reflected pain, we find that their distribution alone enables us to understand the forms assumed on the face by the sensory disturbances due to intramedullary disease.

(b) The remote disturbance of sensation on the side opposed to the lesion may show signs of intramedullary segmentation.

Sensory changes at the level of the lesion might be expected to show signs of a segmental arrangement. But we believe it is also possible to show that, even when the impulses are interrupted after they have passed to the opposite side of the spinal cord, traces of a segmental arrangement can still be discovered. 

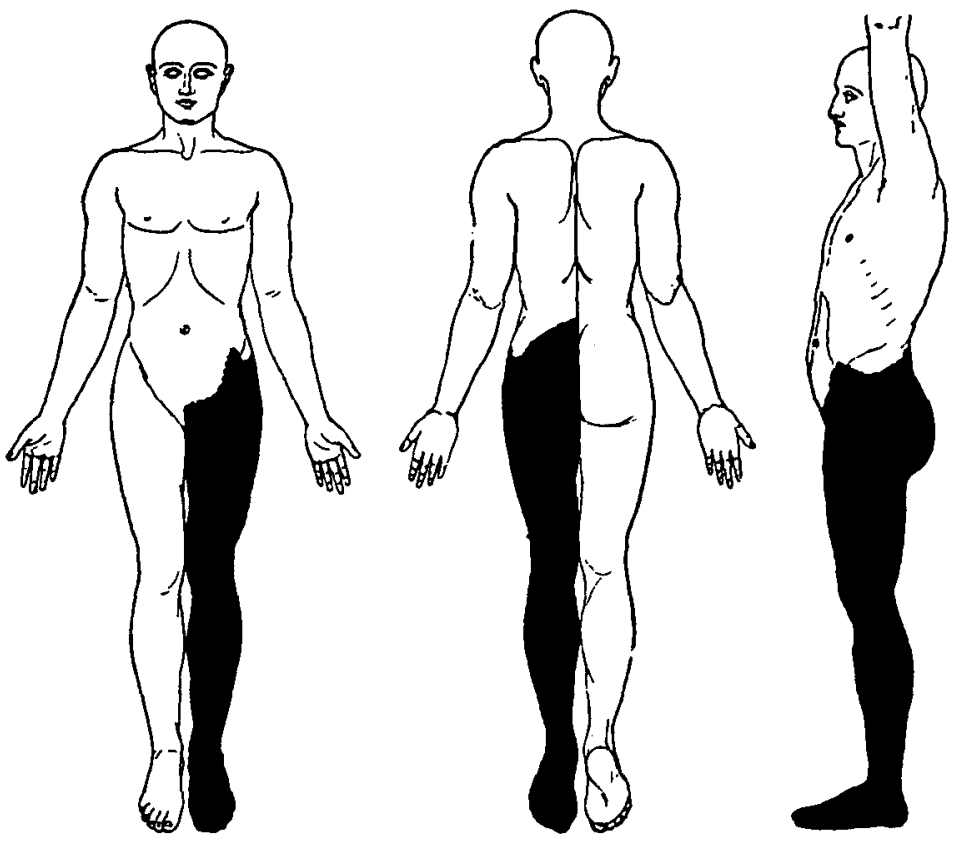

Fig. 36.

To show the disturbance of sensibility to painful and thermal stimulation produced by a lesion which interfered with motion and increased the reflexes in the right.leg. (Case 3, Appendix, p. 656.)

The border between the normal parts and those which were completely insensitive was unusually well defined, corresponding to the caudal border of the eleventh thoracic segment.
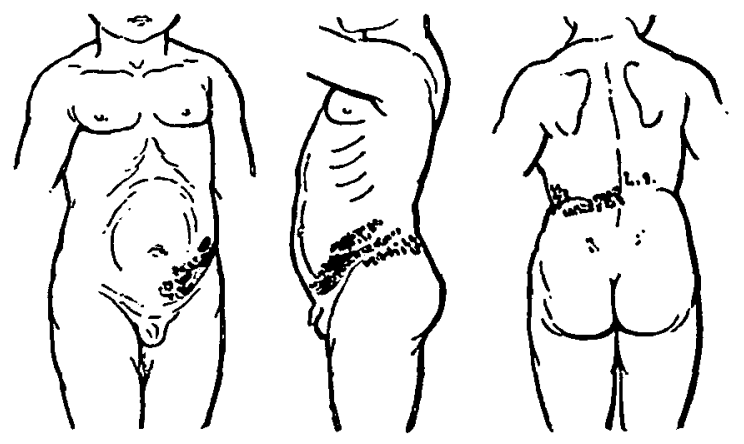

Fig. 37.

Ta show the distribution of the eruption in a case of Herpes zoster proved by post-mortem examination to be due to inflammation of the ganglion of the eleventh thoracic posterior root. (Head and Campbell, Case 5.) 
Thus, in Case 3, the border between parts of normal sensibility and those insensitive to pain, heat and cold was well defined on the side opposed to the lesion. This border resembles the caudal limit of the eruption in a case of herpes zoster due to the destruction of the ganglion of the eleventh thoracic posterior root (Fig. 37). Thus, we must assume that in this case the lesion in the left half of the cord interrupted all the painful and thermal impulses which entered on the right side by the twelfth thoracic posterior root, but allowed all those of the eleventh thoracic root to pass unimpeded.

In the same way, in Case 4, an injury of the cervical portion of the spinal cord produced complete loss of sensation to pain, heat and cold over that half of the body opposite to the paralysis of motion. Here also the boundary between parts of normal and disturbed sensibility was well defined, following closely the caudal border of the first thoracic segment. The injury must therefore have destroyed the paths for all those impulses for pain, heat and cold which crossed the cord in the second thoracic segment, and must have destroyed them after the crossing had occurred.

A similar result was produced by a fracture of the third cervical vertebra (Case 2). Mution was disturbed in the right arm, whilst sensibility to heat and cold was entirely abolished over the left leg, left half of the trunk and part of the left arm. The boundary between parts of normal and abnormal sensibility corresponded to the posterior border of an area frequently marked out by herpes zoster, which Head and Campbell attributed to disturbance of the ganglion of the seventh cervical posterior root.

Now, in this case an exploratory operation proved that the neural arch of the third cervical vertebra had been fractured; and yet the anterior border of the remote loss of sensation on the opposite side corresponded to that of the eighth cervical segment. In the same way, the frontal border of the loss of sensation in W. M. (Case 5) corresponded to the parts which we should expect to become analgesic from destruction of the segments below the second thoracic. But there can be little doubt that the upper,

vol. XxIx. 
rather than the lower, part of the cervical spine was injured by his fall.

Thus, it would seem that, when the loss of sensation (remote) on the opposite sir - to the lesion shows evidence of segmentation, it indicates an interruption of the spinal cord, several segments below the point of injury. This was first shown by Piltz [22], whose statements are borne out by our cases of Brown-Séquard paralysis, as far as they go.

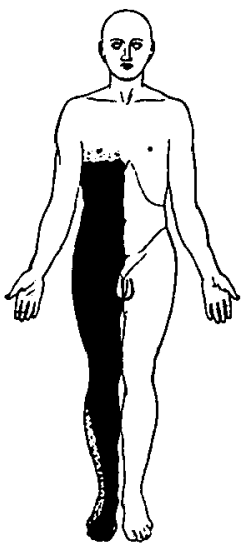

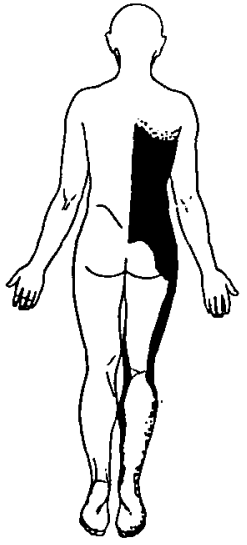

Fig. 38.
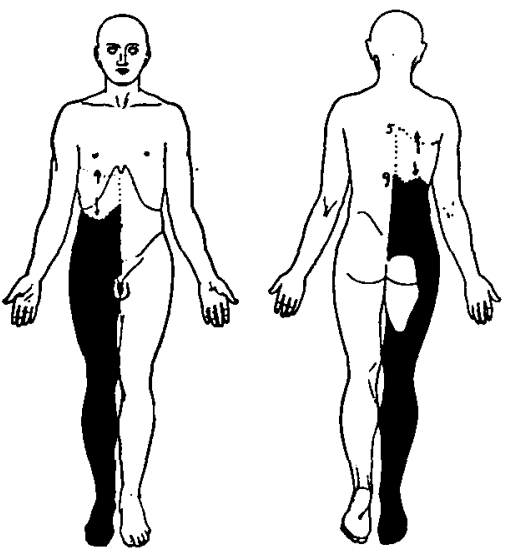

Fí. 39.

To show the disturbance of sensation produced by injury to the cervical spine in Case 6.

Fia. 38.- Shows the extent of the loss to thermal stimuli. Sensation remained intact over the sacral areas.

Fig. 39. - Shows the extent of the loss to cutaneous painful stimuli. Sensation was present over an area corresponding to the lower sacral segmeats only.

(c) The remote analgesia and thermo-anasthesia produced by lesions of the cervical spinal cord, point to a lamellar arrangement of sensory impulses in this region.

Anyone who has studied a long series of cases of spinalcord disease will have been struck with the occasional retention of sensation over the sacral areas, in spite of the complete insensibility of the remainder of the trunk. It would seem that the inpulses fron the caudal end of the 
body may escape an interruption which has overtaken all other sensory impulses.

Sometimes this phenomenon also appears in cases of Brown-Séquard paralysis, and it is particularly well seen in the following instances from our collection. The first of these was a man of 41 years who had injured his neck by diving into shallow water, twenty years before he first came under our care. 'The motor disturbance was represented by distinct wasting of the muscles of the left hand. All loss of sensation was confined to the right leg and right half of the body (Figs. 38 and 39). But, in spite of the widespread cutaneous analgesia, it will be seen that the genitalia with part of the buttock and back of the thigh remained sensitive to prick. The retention of sensibility to heat and cold was even greater, extending to the soles of the feet.

Cyril W. F. (Case 7) showed a similar condition, also produced by diving into shallow water. But, in this case, the loss of sensation was most extensive to heat and of smallest extent to prick (Figs. 9 and 10, p. 559).

It is therefore probable that in the cervical region the impulses from the caudal end of the body (sacral segments), after crossing the cord, pass up in paths separate from those for the sensory impulses from the lumbar and thoracic regions. These paths must be arranged in a lamellar manner, since an injury may interrupt the one set of impulses, leaving the others intact.

\section{Chapter Vi.}

The Passage through the Spinal Cord of Afferent Impulses concerned with "Localisation."

No question has led to greater difference of opinion than the means by which we recognise the position of our limbs in space, and localise a spot stimulated. Fortunately, in. the present paper, we are concerned with the passage through the spinal cord of certain elementary afferent impulses, and not with the question of spacial perception as a whole. This remains a function of the nervous system at a level higher than that with which we are now occupied. 
But, throughout this paper, we have spoken of the power of recognising passive position and movement. Recognition of voluntary movement is a more complex process, based upon impulses grouped differently from those which fall within the range of that level of the nervous system we are now considering. We have also examined the power of naming and indicating the position of a spot touched, and, in every case, we have investigated the capacity for discriminating two points at varying distances. These three tests, the recognition of passive position and movement, the discrimination of compass-points and the localisation of a spot touched, have been chosen, because experience shows that they are affected in lesions of the spinal cord.

We have not only avoided all terms relating to the perceptual aspects of localisation, but also those that express the ultimate sum of many streams of diverse sensory impulses, co-ordinated at a higher level of the nervous system than that with which we are now concerned. We shall use the term " tactile localisation" for the power of discovering the spot to which a stimulus is applied. For those impulses which enable us to recognise the two points of the compasses, we shall use the term "tactile discrimination." Of all those impulses which are concerned in the recognition of articular movement, we shall deal with those of passive movement and position only. ${ }^{1}$ These are the three spinal afferent elements which at a higher level of the nervous system, together with many other factors, lie at the foundation of spacial localisation.

Section 1. The Impulses concerned with the Recognition of Passive Position and Movement.

After division of peripheral nerves, it is found that the power of recognising passive movement and position is closely associated with the integrity of those afferent fibres which run mainly with muscular nerves. It is these fibres

1 Two of these three terms correspond to the groups classified by German authorities as " Raumsinn" (tactile discrimination); " Hautlocalisation" or "Ortsinn" (tactile localisation): Cf. Spearman [32]. 
which innervate the joints, the tendons and the muscles. So long as they remain intact, all cutaneous nerves may be destroyed, and the patient will still be able to recognise the position into wbich his joints have been placed. As soon as these fibres are destroyed, this power is lost. But, in the same path as the impulses for the sense of articular movement and position, travel those which underlie the recognition of deep touch, as far as all subcutaneous structures are concerned. The close association between sensibility to deep touch and the recognition of passive movement and position, is best seen by dividing all the cutaneous nerves to any part, without injury to the muscular trunks. The skin is thus rendered totally anæsthetic, and any sensibility which may remain must be due to impulses which travel in the afferent fibres of the deep system running with muscular nerves. It will then be found that the patient can recognise the tactile and painful elements of pressure, and remains sensitive to passive movement and position of every part of the affected limb, although he is insensitive to every other form of stimulation. Injury to peripheral nerves cannot disturb one of these impulses, without simultaneously affecting others of the group. (Head and Sherren [13], Chap. VIII.)

But, in consequence of lesions within the spinal cord, these impulses can be separately affected. The patient may be sensitive to a deep touch, but at the same time all sensations of articular movement and position may be destroyed.

This was the case in J. Y. (No. 10). A secondary growth within the spinal cord had entirely withdrawn from him all knowledge of the position of his legs. So great was the loss of sensation that he was ignorant even of the grossest changes in their position; and yet he was able to recognise pressure everywhere over both legs.

Conversely, in Case 1, all tactile sensibility and all power of recognising differences in pressure were destroyed over the right leg; and yet, there was no part of the limb where he could not recognise passive position and movement. Passive movements were equally well appreciated, whether the joints moved belonged to one limb or the otber: and 
yet, the right leg was totally insensitive to every other form of stimulation.

With cases of intramedullary disease, it is particularly easy to show that the power of recognising passive articular movement depends upon impulses which are uncrossed and therefore even more elemental than those for pain, heat and cold. For the power of appreciating articular movement and position is always lost on the same side as the disturbance of motion. In Grace G. (Case 3), the left leg was insensitive to all painful and thermal stimulation, but the sense of passive position and movement was perfect. On the right side, sensibility to touch, pain, heat and cold was perfect, but the sense of passive position and movement was disturbed in the right foot. A similar condition existed in Case 2. In consequence of an injury to the cervical spine, this man became insensitive to all painful and thermal stimulation over the left leg and left half of his body. When first seen, the sense of articular movement and position was perfect. But, after an attempt to rectify the condition of his spine, he could no longer recognise the position of his right leg; the surgical operation had destroyed the sense of articular movement and position on the opposite side of his body to the analgesia and thermo-anæsthesia.

This rule, that the sense of articular movement and position is lost on the side of the disturbance of motion, leads to a curious difference between the remote and local sensory phenomena of intramedullary disease. The remote effects consist of loss of sensibility to pain, heat and cold and perhaps to tactile stimuli over the side opposed to the loss of motion; any loss in the sense of articular movement and position will be found in the limbs on the side of the motor disturbance, where the sensibility is otherwise unaffected.

But the local effect of intramedullary disease disturbs cutaneous sensibility on the same side as the motor disturbance, in consequence of an interference with the afferent impulses before they have crossed the cord. The sense of articular movement and position will also be abolished in the same limb, since it is always disturbed on the side of 
the loss of motion. This difference is excellently exhibited by the case of Joseph F. (No. 9). This man was analgesic and thermo-anæsthetic over the right leg, in consequence of disease of the cervical spinal cord. The sense of articular movement and position was disturbed in the toes of the left foot, that is, on the opposite leg to the remote loss of sensation to pain, heat and cold. But this growth in the left half of the spinal cord produced a local loss of sensibility to all forms of touch, pain and temperature in the left arm, and, in addition, he was entirely unable to recognise any movement of this arm, however gross.

In conclusion :

(1) The sense of passive position and movement may be destroyed as a consequence of intramedullary disease, althougb sensibility to deep touch remains perfect. Conversely, the patient may be insensitive to all tactile stimulation, both superficial and deep, and yet may be normally sensitive to passive movements.

(2) Any loss of the sense of passive position and movement will be found on the same side of the body as the disturbance of motion and reflexes.

Thus, the remote effects of intramedullary disease may be manifested in loss of the sense of passive position and movement, disturbance of motion and changes in the reflexes on the one side of the body associated with analgesia, and thermo-anæsthesia of the opposite half.

The local effects of an intramedullary lesion will all be found on the same side; paralysis and wasting of muscles, loss of the sense of passive position and movement, analgesia and thermo-anæsthesia, may all exist together in the same limb.

Section 2.-The Impulses concerned with Tactile Discrimination.

E. H. Weber [39] first employed simultaneous contact with the two points of the compasses as a sensory test. According to him, the power of recognising that a stimulus had an existence external to the body was the highest quality of sensation; sensations grouped together under 
the name of "common sensibility" (Gemeingefühl) did not carry with them this quality. He showed that, when the discrimination of two points was used as a test, some parts were many times more sensitive than otbers; for instance, the two points could not be distinguished over the back, until they were separated to a distance sixty times greater than was necessary for a sensitive surface, such as the tip of the tongue. He believed that, in the compasses, he had discovered a means of measuring the sensibility of the skin, but fully recognised that the test be employed depended upon something apart from crude tactile sensibility.

For, throughout, he speaks of the fineness of the "Ortsinn," and the chapter in which he introduces his experiments is headed "Ortsinn der Haut." His work was imperfectly understood by those who subsequently used the compass-test. Brown-Séquard frankly accepts it as a method of measuring the fineness of tactile sensibility. He does not discuss the method; but, when he wishes to demonstrate the obtuseness to touch of the non-paralysed leg in a case of crossed paralysis, he states that the two points appeared to be one, even when separated to a distance of $13 \mathrm{~cm}$; on the paralysed leg, the patient discriminated the two points, when they were 1.2 or $1.3 \mathrm{~cm}$. apart. Evidently, in this patient, tactile sensibility was gravely diminished, for Brown-Séquard states that "it was necessary to press very hard " on the left leg, "while the patient was able to distinguish the two points, even when they touched but very slightly the paralysed leg." (Brown-Séquard [7]).

Now it will be obvious, that tactile sensibility may be so gravely diminished, that contact may cause little or no sensation. Under such circumstances, the patient would be unable to recognise the two points, because the contact of one or both failed to produce a sensory impulse. The compass-test would then fail from lack of tactile sensibility. ${ }^{2}$

But early in our research on the sensory results of intramedullary lesions, we found that the power of discriminating two points of the compasses might be disturbed over parts,

' Cf. Case 4, p. 662. 
which were sensitive to every other form of cutaneous stimulation. One leg might be insensitive to painful and thermal stimuli, but remain sensitive in a normal degree to the compass-test; the opposite leg might show a gravely defective sensibility to the compasses, although sensitive to every other form of cutaneous stimulation. We also found that, if tactile sensibility was disturbed, the anæsthesia was found over the leg which was also insensitive to pain and temperature. Three facts stood out clearly before us :-

(1) 'The power of discriminating two points can be diminished over a limb completely sensitive to all cutaneous stimuli.

(2) If, as a consequence of the remote effects of an intramedullary lesion, the compass-test shows a diminution of sensibility, whilst touch is perfectly appreciated, the part so affected will lie on the same side as the disturbance of motion.

(3) Whenever tactile sensibility is abolished, in consequence of the remote effects of an intramedullary lesion, this loss of sensation will be found on the side opposed to the loss of motion, over the limb insensitive also to painful and thermal stimuli.

It was therefore evident that, although the compass-test depends upon the integrity of tactile sensibility for its existence, it can reveal a disturbance in sensation which, as far as intramedullary impulses are concerned, is entirely separate from sensations of touch. Throughout this work, we have used the compasses as a means of revealing defects in the impulses, which underlie the power of tactile discrimination, apart from a loss of tactile sensibility. That is to say, we have restored this test to the use originally prescribed by $\mathrm{E}$. $\mathrm{H}$. Weber. We have not used it as a test for tactile sensibility as was done by Brown-Séquard, and, by this strict differentiation, we have been able to reconcile the discrepancies which have so puzzled recent observers. A clear understanding of this use of the compass-test is necessary for the comprehension of the results produced by disease within the spinal cord. For if, among the instances where this test showed some loss of sensation, are included those, 
where tactile sensibility was otherwise defective, the loss of sensation revealed by the compasses will appear, sometimes on the side of the disturbance of motion, sometimes on the opposite side. But, if we confine ourselves to those cases only, where tactile sensibility was perfect, any defect in discriminating two points will be found to lie invariably on the same side as the disturbance of motion and reflexes. ( $C f$. Case 3, p. 656. Case 16, p. 727).

\section{Section 3.-Tactile Localisation.}

We have already considered in this chapter, the effects of an intramedullary lesion on the impulses which underlie the recognition of passive position and movement, and of tactile discrimination. Although they pass to the spinal cord along two afferent paths, these paths become associated within the spinal cord. Not only are the two sets of impulses usually disturbed together by intramedullary lesions, but the loss of sensation to both forms of stimulation is uncrossed, on the same side as the disturbance of motion.

We shall now bring forward evidence to show, that the faculty of tactile localisation is due to impulses which, within the spinal cord, run separately from those of tactile discrimination.

This view was held by Volkmann [38], who first investigated the matter. But the question has been much obscured of late by a misapprehension of the significance to be attached to the compass-test. Some have accepted this test as a measure of tactile localisation, although it was pointed out in 1858, that the compasses tested the "sense of space" (Raumsinn) only. In 1901 Förster [8] went so far as to assert, that tactile localisation depended entirely upon sensations of movement (Bewegungsempfindungen). However much we may differ from this observer in his general conclusions, he was the first to examine a series of pathological cases from this aspect.

Recently, Förster's challenge has been taken up by an experimental psychologist (Spearman [31]). But from a 
clinical point of view, Spearman's case was not completely satisfactory, because the symptoms were evidently bilateral, and sensibility to touch was diminished. We have, how. ever, frequently alluded to this paper, because of the admirable thoroughness of the methods employed.

Before describing in detail the results we have obtained, it is necessary to discuss shortly the methods employed in testing a patient's power of recognising the locality of a spot touched.

First, the patient's eyes are closed and he is asked to say where he is touched. This is the simplest of all methods, and is usually spoken of by us as "spot-naming." If the patient can answer correctly every time he is touched on his great toe, on the heel, on the dorsum of the foot, or on the knee-cap, it is certain that his power of tactile localisation cannot be greatly affected.

If he is asked to point to the spot where he has been touched, he usually errs considerably. But when allowed to grope with the finger he comes closer, and may ultimately touch the very spot. Many observers have pointed out, that this method implies two faculties, a knowledge of the spot touched and of the position in space of the part upon which that spot lies. It requires a combination of the sense of passive position and movement with the power of tactile localisation.

Suppose, bowever, that the sense of passive position and movement is destroyed, and yet the patient can name correctly every spot touched on the limb. It will then be found, that, if he is allowed to grope for the spot, he fails entirely at first to find the limb, but gradually, as testing proceeds, becomes increasingly able to approximate his finger to the spot. That is to say, by means of the touch and movements of his indicating hand, he has partly corrected his defective knowledge of the position of the limb. When this knowledge has been reacquired, move the limb without allowing him to open his eyes. Then he will grope for the spot touched as badly as ever he did, although he still names it correctly.

Spearman devised the following method for overcoming 
this reacquisition of knowledge by means of groping touch. A hole is pierced in a piece of cardboard beld one centimetre from the surface to be tested. Through this hole the patient is touched, and he attempts to point out the spot with a pencil. This marks the card, and the distance of the pencilmark from the hole can be measured. Since the skin is never touched, except by the stimulating object thrust through the hole by the observer, the patient has no opportunity of correcting his defective knowledge by means of touch. This method is an excellent one, where the one limb is affected, and that of the opposite side shows none of the sensory defects with which we are concerned in this chapter. But it only emphasises the double nature of the groping test, which can never be simple so long as we have to deal with the sensory defects produced by an intramedullary lesion.

The power of tactile localisation may remain good over parts where the sense of passive position and movement is entirely absent. In J. Y. (Case 10), who had entirely lost all knowledge of the position of his limbs, we performed the following experiments: His legs were extended in the bed, and he was allowed to see the position into which they had been placed. Then bis eyes were closed and he was touched over the sole, instep and just below the knee cap; in every instance his answers were correct, even ihough the touch was made with cotton wool. With his eyes still closed, the leg was moved into an entirely different position, and his answers were equally correct, although he was entirely ignorant that the leg had been moved, and believed it lay extended before him.

Directly he was asked to point out the spot that had been touched, the double nature of the groping-test was apparent. In the attempt to find the spot he had named correctly, he beat the bed idly, entirely unable even to find his limb.

With the profound disturbance of the power of recognising the position of his limbs was associated an inability to discriminate the compass-points; over the outer surface of the left leg, he failed when they were separated to $15 \mathrm{~cm}$.-

$$
15 \mathrm{~cm} .1 \text { ! } 8 \mathrm{~W} \cdot 2 \mathrm{R} .
$$

and over the front of the left thigh at $20 \mathrm{~cm}$. distance- 
$20 \mathrm{~cm} \cdot \frac{117 \mathrm{~W} .2 \mathrm{R}}{2 \mid 6 \mathrm{~W} .4 \mathrm{R}}$

But, in spite of this failure to discriminate the two points, he could name accurately the spot touched.

Thus, it is evident that, although the impulses underlying tactile discrimination and the sense of passive position are closely associated in the spinal cord, they must be separated from those of tactile localisation as revealed by the power of naming the spot touched.

R. A. H. (Case 16) also showed that the impulses, which underlie the power of naming the spot touched, pass by paths witbin the spinal cord, separate from those for the impulses of tactile discrimination. This man showed signs of chronic intramedullary disease affecting the bulb and cervical spinal cord. The motor disturbance was represented by paralysis of the left vocal cord and left half of the palate, with a spastic condition of the left leg.

There was profound loss of the sense of passive position and movement in the left foot and ankle, and tactile discrimination (compasses) was gravely affected. This case was particularly favourable for such tests, because the sensation of the right lower extremity was unaffected and, on the left leg, painful, thermal and tactile sensibility was normal even to the finest tests. On the sole of the right foot his answers were perfect, when the compass points were separated by a distance of $4 \mathrm{~cm}$. Over the left sole, be gave six false answers at $8 \mathrm{~cm} . \frac{1 / 10 \mathrm{R} .}{2 \mid 4 \mathrm{R} .6 \mathrm{~W}}$.

In the same way, when he was tested by moving his left ankle passively, he failed to give a correct answer in thirteen instances out of twenty. Dorsifexion | $3 \mathrm{~W} .6 \mathrm{O} .1 \mathrm{R}$.

But, with the right ankle, his answers were quick and uniformly accurate.

And yet, in spite of these grave defects in tactile discrimination and in appreciation of passive position and movement, be named a spot touched as accurately on tho left leg as on the right. A number of definite spots on botb lower extremities, such as the ball of the great toe, the heel, 
the external malleolus, were touched with cotton wool ; in no instance did he fail to answer quickly and correctly. Whilst his eyes remained closed, the left foot was placed into an entirely different position. Although he could not tell the situation of the foot, he still named all the points touched as correctly as on the sound side.

But, as soon as he was asked to point out the spot, the difference between the two legs was at once obvious. When tested by Spearman's method, he deviated to the following extent. ${ }^{\prime}$

$\begin{array}{lllcllr}\text { Sole of the foot } & \ldots & \ldots & \text { R. (normal). } & & & \text { L. (affected) } \\ \text { Outer aspect of leg } & . . & \ldots & 4.3 \mathrm{~cm} . & \ldots & \ldots & 7 \mathrm{~cm} \text {. } \\ \text { Front of thigh } & . & \ldots & 2.5 \mathrm{~cm} . & \ldots & \ldots & 6.8 \mathrm{~cm} . \\ \end{array}$

A further series of experiments were made by stimulating the same spot on the dorsum of the foot four times before and four times after movement, the patient's eyes remaining closed throughout. The results were as follows:-

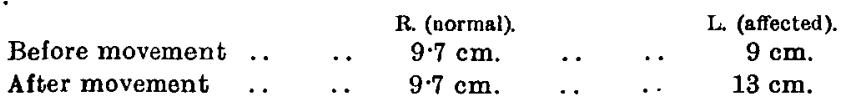

It will be seen that the deviation on the sound side remained the same, but on the affected foot had increased greatly after movement.

From these experiments it is evident, that, at the spinal level, the impulses underlying spot-naming run independently of those for tactile discrimination and the recognition of passive position and movement. But, as soon as an attempt is made to indicate the point stımulated, it is necessary that the patient should recognise the position into which his limbs have been placed passively.

At the spinal level, the impulses for tactile localisation seem to be intimately associated with those of tactile sensibility; we have seen no instance where an intramedullary lesion bas produced defective localisation of touch without a demonstrable reduction of tactile sensibility.

1 In this series he was touched six times at each spot; the numbers represent the average deviation. 


\section{Section 4.-Summary.}

A lesion within the spinal cord can interrupt the afferent impulses which subserve "localisation" in such a way, that the patient fails to answer to one or more of three simple tests. $\mathrm{He}$ may not be able to recognise passive position and movement, he may be unable to discriminate two points separated to a distance far in excess of normal, or he may be unable to name the position of a spot stimulated.

We showed reason to believe, that the impulses induced by the first two tests pass up the same side of the spinal cord as that by which they had entered. The remote sensory loss, produced by the interruption of these impulses, even in the cervical region, is found on the side of the motor disturbance.

In this peculiarity, the impulses underlying the recognition of passive position and movement and of the discrimination of two points, differ from every other sensory impulse passing up the spinal cord. Even the impulses for spotnaming ultimately cross to the side of the spinal cord opposite to their point of entry.

But, with lesions of the cortex and subcortical systems, the loss of appreciation of passive position and movement is found on the opposite side to the lesion. It is obvious, therefore, that all afferent impulses, which reach consciousness and are manifested as a disturbance of sensation, ultimately cross to that half of the nervous system opposed to their point of entry. Some cross quickly; but with some, like those which underlie the sense of passive position and movement, the crossing is delayed until the spinal cord has passed into the medulla oblongata and pons varolii.

Now, sensory impulses which pass up the same side of the spinal cord have not reached the second level of the sensory nervous system. For the most distinctive feature of the impulses at a secondary sensory level is the fact, that the remote results caused by interference with them is manifested on the opposite side of the body.

Thus, it is evident that the impulses underlying the 
appreciation of passive position and movement and tactile discrimination have not attained, within the spinal cord, to the chief characteristic of sensory impulses at the second or intramedullary level of the nervous system.

Each of these two sets of impulses reaches the spinal cord in combination with others. The fibres of the epicritic system conduct, not only the impulses underlying tactile discrimination, but also those for the appreciation of light touch. The peripheral system, associated with deep sensibility, conducts impulses for pressure, for the pain of excessive pressure, as well as those impulses which underlie the sense of passive position.

Thus, by the time the impulses, which pass up in the epicritic system, have reached the cervical region, those for tactile discrimination have parted company with all the otbers, which have passed to the opposite side of the spinal cord.

In the same way, of all the sensory impulses which reach the cord by way of the deep system of nerves, those concerned with the sense of passive position and movement alone remain uncrossed. Those associated with the pain of excessive pressure and with the appreciation and localisation of deep touch, have all crossed to the opposite side of the nervous system within the spinal cord.

Among the many sets of impulses poured into the spinal cord by way of the epicritic and deep systems, a gradual filtering off takes place. At last, in the upper cervical region, none from the epicritic system remain uncrossed, except those concerned with tactile discrimination; none from the deep system, but those which underlie the appreciation of passive position and movement.

When we turn to the impulses underlying tactile localisation, we find that, although they have crossed to the opposite side within the spinal cord, they still remain intimately associated with the impulses for tactile sensibility. The epicritic system brings to the spinal cord the impulses for light touch and cutaneous localisation; the deep system carries those concerned witb the recognition and localisation of pressure touch (deep touch). 
These are immediately transformed and combined into a single group of tactile impulses, which ultimately cross to the opposite half of the spinal cord. Those impulses, associated with tactile localisation, might have been split off to run a course of their own apart from those for tactile sensibility. But this is not the case at the spinal level. Here these two groups of impulses seem to be still intimately associated together. Their fate, at a higher level of the nervous system than that with which we are now concerned, will be the subject of a further communication.

In conclusion, we believe that among the impulses concerned with the local aspect of sensation, which can be interrupted by intramedullary lesions, the three groups do not all belong to the second sensory level. Those concerned with tactile localisation cross the cord and show evidence of recombination; they have, therefore, become sensory impulses of the true intramedullary level. The impulses underlying tactile discrimination and the recognition of passive position and movement show no evidence of recombination, and are interrupted on the same side as motion by intraspinal lesions. From a study of disease higher up the nervous system, above the spinal cord, we know that these impulses ultimately cross, and become recombined. Thus we believe, that a lesion of the spinal cord interrupts these impulses before they have reached the second sensory level. Throughout the spinal cord, they still remain impulses of the primary or peripheral sensory level.

\section{C'hapter VII.}

\section{General Conclusions.}

For fifty years physiologists and clinicians, stimulated by the work of Brown-Séquard, have attempted to trace the upward paths of afferent impulses through the spinal cord. But, in spite of the many admirable experiments on animals and a certain number of well-observed cases of intramedullary disease in man, the latest writers either confess to ignorance, or believe that every impulse passes by at least two paths. 
This widespread scepticism arises from the nature of the methods employed. On the one hand, although the experimenter can produce a lesion of the spinal cord in a healthy animal, which can be killed at any desired period after the operation and the secondary ascending degenerations be traced with exactitude, yet it is impossible to say certainly how sensation is affected by the lesion, even when the animal has been long under observation.

On the other hand, the clinician may expend much time and trouble in determining the nature of the loss of sensation; but it is especially in these well-observed cases that it is usually impossible to verify the anatomical nature of the lesion. Most of the instances, in which a careful microscopical examination has been possible, have been cases where the lesion has been progressive, or where the patient has died from complications which greatly detracted from his suitability for psychophysical examination.

No one who has read our paper will think we do not value the experimental or clinical work of our predecessors. If we may seem to pay less detailed attention to it than is customary, it is solely because we have attacked the problem from a different aspect. We accept the experimental result that in animals it is impossible to abolish the upward passage of all afferent impulses from one limb by hemisection of the spinal cord, and fully recognise that the majority of clinical reports are anatomically unsatisfactory.

Recognising the inherent difficulties of both methods, we determined to attack the problem from a different aspect. All previous workers have assumed that afferent impulses reach the spinal cord in the same combinations as those ultimately received by the centres in the brain. Whatever psychical sorting might take place among sensations, it has been assumed that the sensory impulses pass from the periphery to the highest physiological centres, unchanged in quality and in unaltered combinations.

But Head, Rivers and Sherren showed that this could not be the case; for the afferent impulses passed up the peripheral nerves in combinations entirely foreign to those familiar to the investigators of normal sensibility. They 
showed that sensory impulses reach the spinal cord by way of the fibres of the posterior nerve-roots in the same remarkable combinations. It was, therefore, certain that the grouping of these impulses must have changed in some way. Where this change occurred, and how the sensory impulses were combined anew, was the problem we set before us.

\section{(a) Results obtained by the Physiological Method.}

Our nethod has been to trace known sensory impulses, arriving by way of normal peripheral nerves and posterior roots through a diseased spinal cord to higher centres that are entirely unaffected, and we have attempted to indicate how sensation is modified under such conditions.

We showed in Chapter II., how the disturbance of sensation produced by an intramedullary lesion differed from that due to division of peripheral nerves. Impulses arrive by three main streams, along the fibres of the protopathic, epicritic and deep systems. We compared this grouping of impulses at the primary, or peripheral, level with the changes in sensation produced by a purely intramedullary lesion; and for this purpose, we chose the loss of sensibility on the opposite half of the body to that on which motion and reflexes were affected.

All sensory impulses which have crossed the cord show signs of recombination. The form assumed by the loss of sensation shows that the tactile elements have become united into a single group, by whatever peripheral path they may have reached the spinal cord. In the same way, the impulses started by cutaneous painful stimulation arriving by the protopathic fibres, and those due to painful pressure arriving by the deep system of nerves, become combined, parting company with all other sensory impulses that may have travelled with them in peripheral paths. The thermal impulses of the epicritic and protopathic systems become recombined, so that an intramedullary lesion can interrupt all those subserving the sensation of heat, without interfering with the impulses underlying sensations of cold. Or, in some cases, the patient may be insensitive to cold over parts completely sensitive to heat of all degrees. In 
passing from the peripheral to the intramedullary, or secondary, level, the impulses change from a regional and developmental grouping to physiologically specific combinations.

Finally, we analysed the loss of sensation caused by intramedullary disease at the level of the lesion, and showed that, in character, it agreed with that remote loss on the side opposed to the lesion where the sensory impulses bad been interrupted after they had crossed the spinal cord. Thus, the change must bave occurred on the same side of the cord as that by which the sensory impulses entered.

\section{(b) The Nature of the Impulses in the Posterior Columns of the Spinal Cord.}

Tactile, painful and thermal impulses, and those associated with tactile localisation, cross in their passage through the spinal cord, and show evidence of recombination. But. the sensory impulses which underlie the recognition of passive position and movement and tactile discrimination do not cross within the limits of the spinal cord. Lesions of the higher centres show that, ultimately, even these impulses cross and are grouped anew; but this change cannot be demonstrated by a pure intramedullary lesion.

Injury and disease of the cervical region of the spinal cord prove that an impaired tactile discrimination and loss of appreciation of passive position and movement may form the only remote loss of sensation on the same side as the disturbance of motion. Thus, although these impulses show no signs of recombination and are still impulses of the lowest sensory level, they are no longer associated with the sensory companions with which they travelled in the peripheral nerves. The impulses underlying tactile discrimination, which reached the spinal cord by way of the epicritic fibres, have parted company with the impulses for light touch, for tactile localisation and for intermediate degrees of heat and cold. The deep system of afferent fibres running with muscular nerves, carried to the spinal cord, not only the impulses associated with the recognition of passive position and movement, but also those for the appreciation and localisation of deep touch and for the pain of exces- 
sive pressure. Of epicritic impulses, those for tactile discrimination alone remain uncrossed and uncombined in the cervical spinal cord ; similarly, of all those which passed into the spinal cord by way of the deep system of fibres, the impulses underlying the appreciation of passive position and movement alone remain uncrossed and uncombined.

For these impulses we must therefore seek some path in the spinal cord, which is the direct continuation of the posterior roots and which remains uncrossed, until we have passed beyond the limits of the spinal cord. Such a path is found in the long extrinsic tracts of the posterior columns. Fibres from the fifth lumbar and first sacral posterior roots can be shown to degenerate up to the nucleus of the posterointernal column, and degeneration can be traced up the complete length of the postero-external column, after division of the posterior roots supplying the hand. But the number of fibres which can be found degenerated in the upper part of the cervical region is but a small fraction of those which show evidence of destruction, as a consequence of division of any one posterior root. All the way up, the posterior columns are giving off fibres to other parts of the cord.

On tracing the sensory impulses throughout their course in the spinal cord, an exactly analogous filtering off occurs. Painful, thermal, tactile impulses ultimately pass from the point of entry to the opposite side of the spinal cord.

But, before they pass across, they have undergone characteristic recombination. That this change occurs in the same half of the spinal cord by which the impulses enter, is shown by the fact that the local loss of sensation produced by a pure intramedullary lesion closely resembles, in character and grouping, that of the remote sensory disturbance which lies over the opposite half of the body.

The rapidity with which the sensory impulses cross to the opposite side varies greatly. Some, such as those associated with pain, heat and cold, seem to have passed completely to the opposite side in the space of five or six segments (Piltz [22], vide also Chap. V, p. 617). Thus, in man, an intramedullary lesion may interfere completely with sensory impulses for pain, heat and cold from the opposite 
half of the body, without disturbing any other form of sensation. With tactile impulses, the crossing is evidently less rapid; for it is rare to find a remote disturbance of tactile sensibility. But, ultimately, even tactile impulses pass completely to the opposite side of the cord, and a lesion in its upper part may, if sufficiently severe, produce loss of tactile sensibility over the same parts that are analgesic and thermo-anæsthetic. But, until this crossing is finally completed, it is obvious that two patbs will be open for tactile impulses. They will continue to pass up the fibres of the epicritic and deep systems in the posterior column, until the highest point in the spinal cord at which those impulses entering by any one posterior root are received by the secondary systems. But, much nearer the point of entry, some impulses will have become transformed and have crossed to the opposite side. Thus, two paths will be open for tactile impulses, one in the primary system of the posterior columns, the other in the secondary intramedullary system. In the first of these paths, the impulses travel untransformed, whilst as soon as they enter the secondary path, they will have been recombined into a specific group, associated with all forms of tactile sensibility.

For the impulses associated with pain, heat and cold, a primary and a secondary path coexist for a short extent only within the spinal cord; for those associated with all forms of touch, this double path seems to extend over a considerable length. Finally, the impulses associated with passive position and movement and with tactile discrimination do not, within the limits of the spinal cord, reach the point where they are recombined, but continue uncrossed to pass along the fibres of primary afferent systems in the posterior columns. It is not until they reach the posterior column nuclei (nucleus gracilis and nucleus cuneatus) that they pass from a primary to a secondary sensory system. Here, at last, the impulses associated with tactile discrimination and with passive position and movement undergo recombination, and cross to the opposite side of the nervous system.

Epicritic and protopathic impulses, and those associated 
with deep sensibility, travel along the fibres of the posterior columns like rubble over a graduated sieve. Stones of small size drop through at once, those that are larger pass on further, and some travel the whole length of the sieve to fall into a heap at the end. So, some impulses cross rapidly, others after a longer course; some do not become sifted off until the cord has terminated above in the nuclei of the posterior columns.

These impulses alone can be completely interrupted by destruction of the posterior columns. We therefore find, that the only definite consequence of destruction of the posterior columns is to produce loss of tactile discrimination [compass-test] and of the sense of passive position and movement on the same side as the lesion.

(c) The Significance of the Delayed Crossing of Sensory Impulses of the Secondary or Intramedullary Level.

What is the significance of this delayed constitution of the crossed tracts of the secondary or intramedullary level? The impulses which result from all forms of painful stimulation pass to the spinal cord by the protopathic fibres, and by the fibres of the deep system which run with the nuuscular nerves. The impulses from the skin, arriving by way of the protopathic system, probably become almost at once transformed, and pass into an intramedullary system at the level of their point of entry. It is probable that the fibres of the deep system, carrying impulses produced by painful pressure from the same part of the body, do not enter by the same posterior roots as those carrying the impulses produced by cutaneous painful stimuli. Thus, more than one segment of the cord is required, before all the painful impulses from any one part of the body can be gathered together and recombined.

This is probably the cause of that want of correspondence between the extent of the cutaneous and deep analgesia, produced by the local manifestations of an intramedullary lesion (vide p. 595). It also underlies the remarkable condition described on p. 597, where an area of pain and hyperalgesia to the lightest pressure, due to a diseased joint, lay within the limits of total cutaneous analgesia. 
Such cases show why a certain extent of the spinal cord must be traversed before the transformed impulses from any part of the body can be gathered up in a path on the opposite side of the spinal cord. Interruption at any point after they have crossed may produce a remote analgesia with a definite upper border, but this border will probably lie several segments below the position of the interrupting lesion.

An even greater extent of the spinal cord must be traversed by the impulses associated with light touch arriving by way of the epicritic fibres, and by those for sensations of pressure conducted along the deep system. For, the extent of the epicritic supply of any one posterior root greatly exceeds that of its protopathic fibres; the overlapping is greater, and the epicritic fibres are less completely segmental in distribution.

The local effects of an intramedullary lesion are produced by interference with the paths of the secondary level before they have crossed the cord, whereas the remote effects are due to interruption of these tracts as they pass up towards the third level after crossing the cord.

Disease of the grey matter as such does not produce loss of sensation, except by interfering with these patbs of the secondary intramedullary level as they pass through its substance.

The more nearly segmental the arrangement of a primary sensory system as it enters by the posterior roots, the shorter will be the distance necessary for the complete crossing of the impulses it carries into the spinal cord. Thus impulses for pain and sensations of temperature arising mainly out of highly segmented protopathic impulses have the shortest double path.

(d) The Path of Impulses Subserving the Sense of Passive Position and Movement.

We have shown that the more nearly a primary sensory system is arranged segmentally as it enters the spinal cord by the posterior roots the more quickly will its impulses cross to the opposite side after recombination. 
The fibres which conduct the impulses for passive position and movement are probably arranged on a plan different from that of the remaining sensory segmentation. The greater number start from end-organs in the joints, muscles or tendons of the limbs, and in the leg, chiefly from the foot and ankle. When the sense of passive position and movement is disturbed, the loss of sensation is arranged according to joints.

Thus, we should not expect the crossing of these impulses to occur as rapidly as those subserving thermal and painful sensibility. In fact, we find that complete crossing and recombination does not occur, until after these afferent impulses have reached the posterior column nuclei.

But this delay in crossing and recombination of the impulses subserving the sense of passive position and movement, seems to have another cause. We have investigated those afferent impulses only which reach consciousness; but many afferent impulses pass in by the primary or peripheral systems which never produce sensation.

We wish to emphasise a distinction between the fate of sensory and non-sensory afferent impulses, which has not been insisted upon sufticiently of late years. If a tract is shown to degenerate from below upwards, it probably conducts afferent impulses. These impulses may however never reach consciousness; they may be associated with some higher reflex condition, such as the maintenance of equilibrium, or the tone of muscles. This may be an entirely unconscious process, and sensation may never be evoked, until some disturbance of equilibrium produces impulses which, passing up sensory paths, reach the highest centres concerned with sensation. A disturbance of nonsensory afferent impulses produces an abnormal condition in the organism. This abnormal condition evokes fresh afferent impulses which, passing up sensory paths, causes the organism to become aware of the change that has occurred in a state normally outside consciousness.

Now, the afferent impulses, passing by means of the deep system from tendons and joints, are pre-eminently associated with the higher reflex states, such as equilibrium. Recent 
work, especially that of Horsley and of Sherrington, has shown that the cerebellum is the afferent centre for these bigher reflexes. Any column, therefore, which conducts impulses from the tendons and joints will probably give off collaterals to afferent cerebellar tracts.

One such patb, the direct cerebellar tract, lies on the same side as the posterior roots by which the impulses from the deep system enter the spinal cord.

It is therefore probable that, in their passage through the spinal cord, the afferent impulses of deep sensibility, concerned with the position and movement of joints, divide into two groups-those destined after transformation to become the non-sensory afferent impulses of the direct cerebellar tract, and those which continue up the fibres of the primary system in the posterior columns to subserve the sense of passive position and movement. The latter impulses do not become recombined until they reach the nucleus gracilis and nucleus cuneatus; they then cross rapidly to the opposite side like every other sensory impulse.

\section{(e) The Theory of Intramedullary Specific Receptors.}

All sensory impulses are ultimately recombined, on passing from the peripheral to the intramedullary level. This recombination takes place in the same half of the nervous system as that by which the sensory impulses enter. Impulses from the primary level which have been initiated by painful stimuli of whatever kind, are united into a single group. In the same way, all tactile impulses, whether they arrive by way of the epicritic or deep systems, are gatbered up and travel together in the secondary system.

We believe this is due to an arrangement somewhat as follows; each end-organ in the skin is capable of reacting to the mass stimulations of the environment in a specific manner ; hot spots react to certain degrees of heat only, cold spots to cold or, paradoxically, to heat above $45^{\circ} \mathrm{C}$. The endorgans of the deep system react to pressure, whether it be painful or not, but do not respond to stimulation with heat and cold. 
When these peculiar impulses reach the spinal cord, they are discharged into the secondary systems, each of which is guarded by specific receptors. These act towards the impulses of the primary sensory level exactly as the end-organs in the skin acted towards the mass-stimuli of the environment. When an impulse, which has originated in the effective stimulation of a heat spot belonging to the protopathic system, reaches an intramedullary receptor of the secondary system set aside for the impulses of heat, it starts a specific impulse. But the same receptor reacts to the epicritic impulses which are started by stimulation of the skin with temperatures between $34^{\circ}$ and $40^{\circ} \mathrm{C}$. No reaction, however, occurs if the receptor of the secondary system is exposed to impulses due to any other form of stimulation.

Similarly, the receptors of the secondary system arranged for painful impulses respond to all impulses of this nature, whether they arise from stimulation by a prick, or from excessive pressure.

In the same way, all tactile impulses are gathered up by the receptors of the secondary tactile system, whether they are started by a light touch and arrive by way of epicritic fibres, or by pressure and reach the spinal cord through deep afferent fibres running with muscular nerves.

Thus, we imagine that, at the point where sensory impulses pass from the primary to the secondary level, there is a mechanism capable of responding to one specific sensory impulse, however that impulse may have been originated.

It is as if the gallery of a concert hall were fitted with a series of resonators, each of which was tuned to a certain note. Each resonator would pick up a peculiar tone, whether it was produced by the strings, the brass, or the wood-wind.

\section{(f) Sensory Impulses of the Secondary Level undergo further} recombination at the Third Sensory Level.

When sensory impulses pass from the primary or peripheral to the secondary or intramedullary level, they are 
recombined in such a way that those which bave been initiated by painful, thermal or tactile stimuli are brought together into specific groups. Interruption of sensory paths within the spinal cord produces a loss of sensation so nearly specific in character, that it might be thought no further recombination was possible.

Closer examination of the groups of sensory impulses at this level shows that they have still to undergo further sifting; the nature of this change in grouping at the tertiary level will form the theme of a further communication. It must be remembered, that intramedullary tactile impulses are still closely associated with those of tactile localisation, and no case has come under our notice where the patient was unable to name the spot touched, unless at the same time tactile sensibility was distinctly diminished. This is a relic of the close relation of sensibility to light touch with cutaneous localisation, and of sensibility to pressure with the localisation of deep touch. Finally, at the tertiary level, all the impulses subserving localisation are brought together, and the impulses for tactile localisation become separated from those for contact sensibility.

Moreover, at the second or intramedullary level, specific sensory impulses carry with them the factors which determine the feeling-tone of the sensation they ultimately subserve. When a strong interrupted current is applied to the foot, the patient cries out with pain and withdraws the leg. Suppose, however, that the lower extremity is totally insensitive to painful stimulation of all kinds, in consequence of an intramedullary lesion, the current, even when of excessive strength, cannot cause pain. But so long as tactile sensibility remains perfect, he will complain bitterly of the discomfort caused by this form of stimulation.

Careful experiments with G. G. (No 3, p. 656), and W. M. (No. 5, p. 667), both of whom were unusually trustworthy and willing patients, showed that the movement of withdrawal seemed to be almost as violent when a current of known strength was applied to the analgesic as to the normal leg. G. G. said the sensation produced was a " kind of exaggerated tickling more unpleasant than pain." Both these patients 
were firm in their assertions that the sensation was not painful; and yet, an observer watching their behaviour would suppose they were undergoing intolerable pain. This reaction was not evoked on the analgesic side by a purely painful stimulus such as the prick of a pin.

Evidently, stimulation with a strong interrupted current normally causes pain, which rising to intolerable limits obscures all other sensations. But, if the painful impulses are interrupted by an intramedullary lesion, the tactile side of this stimulus is revealed, and, when the current is strengthened, can produce a sensation painless, but intensely disagreeable.

Thus, at the second, or intramedullary level, even the impulses of tactile sensibility can subserve a discomforting sensation.

\section{(g) Conclusion.}

In conclusion, we believe that the spinal cord is the seat of the transmutation of most of the impulses of the peripheral into those of the secondary level of the afferent nervous system.

This transmutation and recombination takes place on the same side as that by which the impulses enter the spinal cord. The secondary paths for sensory impulses then cross with greater or less rapidity, so that ultimately all except those subserving the sense of passive position and movement and tactile discrimination have passed to the opposite side within the limits of the spinal cord. Even these sensory impulses cross after reaching the nuclei of the posterior columns.

At the same time, within the spinal cord afferent impulses become separated into sensory and non-sensory. Of the latter, many pass up in the secondary system of the direct cerebellar tract to reach the cerebellum.

Thus, the mechanisms of the secondary or intramedullary level are concerned with the separation of non-sensory from sensory afferent impulses, and with the recombination and transwutation of sensory impulses into specific groups. 


\section{APPENDIX.}

Reports of Cases quoted in the Text.

\section{Case 1.-Frederick C.}

He was suddenly attacked with wenkness of the legs and left arm, December 13, 1903.

On Jan. 4, 1904, he showed weakness of left arm and disturbed sensibility over the right half of the body and right leg.

Seen by us August, 1905 . Well compensated mitral regurgitation.

Wasting of small muscles of left hand.

Knee-jerks brisk, no ankle-clonus, left plantar reflex gave a flexor response.

Total insensibility to tactile, painful and thermal stimuli was present over the right leg and right half of the trunk (fig. 40).

Frederick C., aged 37, a coal porter, went to bed perfectly well on the night of December 13,1903. When he tried to get out of bed at six o'clock next morning, he found that he could not use his legs. He fell to the ground and had to be helped back to bed. The same afternoon he discovered that his left arm was useless. He was nursed at home for a fortnight, and the weakness of the legs disappeared; but he was still unable to use bis left arm. On January 4, 1904, he was admitted as an in-patient to the National Hospital, Queen Square, where he remained for five and a half weeks. His condition was then as follows: "There was weakness of the left arm and loss of sensation over the right leg and the right side of the trunk; the condition was one of complete analgesia and thermo-anæsthesia, with relative loss of sensibility to touch over this half of the body." 1

He improved greatly whilst in the hospital, and returned to work on the Tuesday after Easter, 1904. The weakness of his left arm had disappeared to such an extent, that he was able to carry nine tons of coal on the first day he resumed work. Soon, however, he began to notice that sensation was lost in the right

\footnotetext{
1 For this note we are indebted to the Registrar of the National Hospital, Queen Square.
} 
leg. Pieces of coal falling upon it caused him no pain, and be was often unaware that he had been struck, until he saw bruises on the leg at night. Further, he found that the leg was insensitive to pinching. He did not trouble himself about this loss of sensation, but on August 13,1905, he began to have pains in his joints, and was admitted to the London Hospital, on August 16, with an attack of acute rheumatism.

When he was 20 years old, and again at the age of 31 , he had had attacks of rheumatic fever, and, for the last twelve months, had been a little short of breath on exertion. He denied syphilis and gonorrhœa. He married at 21 years old, had one child, aged 2 years, who is quite healthy. His wife had previously had three miscarriages.

His work was heavy, as he had not only to drive the van, but also to load and unload the coals. He drank about ten pints of beer daily, but no spirits, and smoked about an ounce of shag a day.

There was no family history of nervous or rheumatic disease. He was liable to urticarial wheals, and factitious urticaria could be produced by pricking or scratching the skin all over the body, both over anæsthetic and over normal parts.

The day after admission, his temperature was $102^{\circ} \mathrm{F}$. [38.8 $\left.8^{\circ} \mathrm{C}.\right]$, and he had a good deal of pain in the left knee, the left ankle and in both shoulders. He also experienced an uncomfortable sensation in the right knee, and he objected to flexion of the joint, but stated that the sensation was not at all painful ; it resembled an itching and was very disagreeable.

The temperature and pain rapidly subsided under the influence of sodium salicylate.

The heart was somewhat enlarged and showed the signs of mitral regurgitation of moderate severity. Compensation was well maintained.

Since this date, the patient has been under frequent observation, and in the following account we shall summarise his present condition.

$\mathrm{He}$ is a tall, spare, but very muscular and powerful man, intelligent and answering readily to the various forms of stimulation. His speech is normal, and he has never suffered from fits.

Motion.-The grasp of the left hand was a little weaker than that of the right, but this difference was much increased during the attack of rheumatism, when the dynamometer readings were:- 
He was examined on many occasions after the rheumatism had passed away, and the average results were as follows :-

Right grasp 30 kilograms.

Left grasp 23 kilograms.

There was slight but distinct wasting of the first dorsal interosseous muscle and of the muscles of the thenar eminence of the left hand. All the movements of the hands, forearms and arms
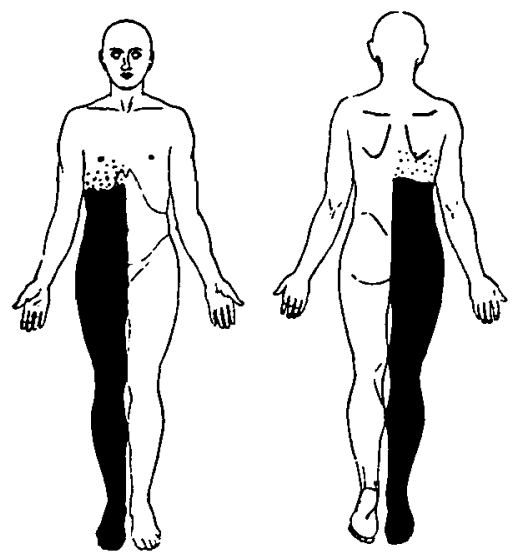

Frg. 40.

To show the loss of sensation to all forms of tactile, painful and thermal stimuli in Case 1. The frontal border was not sharply defined, but merged gradually into parts of normal sensibility.

could be readily performed, and no wasting could be detected in the remaining muscles of either arm. The gait was normal. He did not drag either leg, and it could not be said that one leg was held more stiffly than the other. He could stand steadily with both eyes closed, and even balance on either foot with equal ease. There was no wasting or tremor in either leg. He could approximate his great toes with his eyes closed, even when he had not previously seen the position of his limbs.

The electrical reactions of the muscles in both arms and legs were normal.

Reflexcs.-The knee-jerks were brisk, normal and equal, and the plantar-reflex, although diffcult to obtain, seemed to give a normal flexor response on both sides. 
Sensation.-F. C. stated that the right leg always seemed to him to be hot, as if it were before a blazing fire. When he placed the left leg upon it, or when he touched the right leg with either hand, the right leg always seemed to be hotter than the left.

Pain-(a) Stimulation by pricking with the point of pin elicited no reply over the whole of the right leg and over the right side of the body as far as the level of the xiphisternum. The upper border was a soft one and shaded gradually into parts of normal sensibility. The anæsthesia was limited exactly to the middle line and included the skin covering the rigbt half of the penis and the right side of the scrotum.

No pain was produced over the right leg by pressure which would have been unendurable over the left leg. The right side of the body as far up as the level of the cutaneous analgesia was also insensitive to the pain of deep pressure. Not only was the pain of deep pressure lost, but the patient also failed entirely to recognise any increase in pressure even when 15 kilograms were used. The algometer records are given below.

\section{ALGoNeter.}

\begin{tabular}{|c|c|c|c|c|c|c|c|c|c|c|}
\hline & & & & & & & & R. & & \\
\hline Sole & .. & .. & .. & * & .. & .. & 15 & [no pain] & .. & $5[\mathrm{P}$ \\
\hline hin & .. & .. & . & . & $\cdots$ & . & 13 & [no pain] & .. & $2 \cdot 5[\mathrm{ps}$ \\
\hline uter si & of & & .. & . & . & & 20 & [no pain] & .. & $4.5[\mathrm{p}$ \\
\hline ront & hig & & & . & .. & & 15 & [no pain] & & $5 \quad[\mathrm{p}$ \\
\hline uter & $\mathrm{ce}$ & the & nt. & il & spine & & 15 & & & \\
\hline ow & & in th & imi & & 8.1ge & & 10 & [no pain] & & $1.5[\mathrm{p}$ \\
\hline & & $r$ jo & .. & .. & .. & .. & 3 & [pain] & & 3 \\
\hline & .. & $\therefore$ & .. & . & . & $\ldots$ & 2 & [pain] & . & 2 \\
\hline ow & & . & .. & .. & .. & .. & 4 & [pain] & & 4.5 \\
\hline
\end{tabular}

He was insensitive to the interrupted current in all its forms over the whole of the analgesic half of the body and over the right leg. He could appreciate neither the pain nor the vibration of the current. But, as soon as the muscles were contracted so as to produce movement of a joint he said, "You are doing something to me." This occurred every time that any considerable move. ment of a muscle was induced.

(b) Thermal stimuli.- $\mathrm{He}$ was insensitive to stimulation by water at $50 \mathrm{C}^{\circ}$., or by ice, over the whole of the right leg and right half of the body so far as the level of the xiphisternum. The upper level of the thermo-anæsthesia was not sharply defined, and there was an area of skin extending upwards for about an inch and a half over which sensibility to temperature 
was diminished. The thermo-anæsthesia was limited exactly by the middle line. Over this area, stimulation with the intermediate degrees of temperature likewise failed to evoke any response.

(c) Tactile sensibility.-There was profound loss of sensibility to cotton-wool over the whole of the right leg and right half of the body, the upper limit extending to nearly the same level as that of the analgesia and thermo-anæsthesia.

On testing the two legs with von Frey's hairs, it was found that No. 8 [830 mgm.] was everywhere promptly appreciated and accurately localised over the whole of the left leg. No contact with any of these hairs could be appreciated anywhere over the right leg as far as the upper limit of the anæsthesia to stimulation with cotton-wool.

Pressure steadily applied evoked no response over the whole of the right leg and of the lower half of the trunk on the right side. Over the right thigh and over the lower part of the abdomen on the right side, however, if the instrument by which pressure was made was moved sufficiently violently to displace the muscles, the patient stated that he felt a slight sensation. If the whole of the skin and subcutaneous tissues were picked up between the finger and thumb and violently pinched, he never on any occasion gave any response. The sensation experienced by the patient seemed to depend on the movement of the muscles.

(d) Tactile localisation.-Localisation of touch was perfect over the whole of the left leg, whether the patient was asked to name the point touched with his eyes closed, or whether he was allowed to grope for the point of contact. The maximum final error was in all cases under $2 \mathrm{~cm}$.

(e) Compasses. - Over the right leg it was impossible to apply this test, owing to the fact that the patient did not respond at all when touched with the compasses, in consequence of the loss of tactile sensibility.

Over the left leg the results were as follows :-

Outer side of left leg (longitudinal).

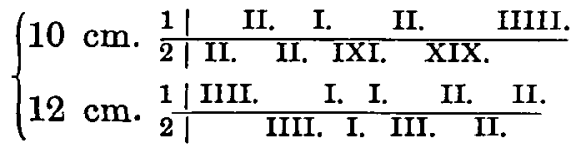

The answers were given promptly and with decision. On the right half of the abdomen a similar difficulty was encountered, since the patient did not respond at all over this area when 
touched. Over the left half of the abdomen the result was as follows :-

$\left.\begin{array}{l}\begin{array}{l}\text { Left half of abdomen } \\ \text { (longitudinal). }\end{array}\end{array}\right\} 6 \mathrm{~cm} . \frac{1 \mid \text { III. II. III. II. }}{2 \mid \text { II. III. II. II. }}$

On the hands compass sensibility was normal.

$(f)$ Sense of passive position and movement.-Although there was profound loss of cutaneous and of tactile sensibility in the right leg, the patient was able to recognise any position into which his limb was passively placed. The results obtained were as follows :-

$$
\begin{aligned}
& \text { Great Toe } \frac{\begin{array}{l}
\text { Rigtension | } \\
\text { Flexion }
\end{array} \text { |II. I. II. II. II. II. III. II. }}{\text { II. }}
\end{aligned}
$$

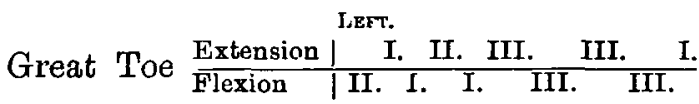

There is a little stiffening of the great toe joint which prevents the answers being as good when the toe is extended as when it is flexed.

All movements at the ankles and knees were appreciated with equal accuracy.

Although the patient could not appreciate firm, steadilyapplied pressure, he experienced a sensation if his muscles were seized and moved about. If the muscles were made to contract by an electric current, he appreciated the fact that they were being moved. Moreover, he was able to co-ordinate the movements of the muscles of the right leg quite as well as those of the opposite sound limb.

(g) Acuasthesia and sense of size.-As might have been expected from the total loss of painful and tactile sensibility, the patient was quite unable to distinguish the head from the point of a pin, or to discriminate between dises of various sizes when applied to the skin of the right leg.

(h) Tuning-fork.-All sensation of vibration was lost over the right leg, the right iliac crest, and the right half of the abdomen. The vibrations were, however, appreciated over the lower ribs and the costal margin on the right side.

Cranial nerves.-The pupils were equal and reacted well to light and accommodation." No cranial nerve was affected.

Sphincters.-There was no abnormality in micturition or defæcation.

Spine.-No deformity. 


\section{Case 2.-Charles B.}

Fracture of the neural arch of the third cervical vertebra from a fall on to the head.

Loss of sensation to pain and temperature over the left half of the body. (Fig. 41.)

Sensation to all forms of touch undisturbed.

Sense of passive position and movement, tactile discrimination and tactile localisation perfect.

Laminectomy, June 25, 1905.

After the operation the condition of sensation was unaltered, except that all sense of passive position and movement was lost in the right arm and leg.

On April 11, 1904, Charles B., a man of 59, was thrown out of a dog-cart on to his head. He thinks he lost consciousness, but is certain that such loss was momentary only, for he remembers lying in the road, watching all that was going on around him, completely paralysed down the right side. He was carried to bed and was able to move his toes upon the tenth day after the accident. Power gradually returned to the right leg, and in two months the right arm was so far recovered that he was able to write.

From the moment of the accident he has been insensitive to heat and cold over the left half of the body. From the left hip downwards he has suffered from a burning sensation, which seems to lie deep below the surface and is always worse after a good meal. He has also suffered greatly from cramp in the right leg.

Condition in May, 1905.

He was a spare, well-built man, looking fully his age. His vessels were not abnormally thickened, and the heart and lungs showed no disease.

Motion.-He walked like a man with hemiplegia, lifting the right hip, and swinging the right foot, so that the toes cleared the ground. He could stand steadily with his eyes closed even when his feet were placed together. As he lay on a couch, there was very little loss of power in the right foot or right knee, when the movements were made against resistance. He could dorsiflex the right foot at the ankle, but the movement was somewhat slower than on the opposite side.

The grasp of the right band was decidedly weaker than that of the left; all movements at the right wrist and elbow could be 
performed, though they were less strong than on the opposite side. He could touch his nose with the forefinger of either hand, even when the eyes were closed, and showed no signs of ataxy. The supraspinatus, the infraspinatus and deltoid muscles were slightly wasted; but it must be remembered that the right shoulder joint was fixed by adhesions. The action of the deltoid was weak, but the biceps and supinator longus contracted strongly ; none of the small muscles of the hand were wasted. All the muscles reacted perfectly to the interrupted current, even those that appeared to be wasted.

Reflexes.-The right knee-jerk was greatly exaggerated. Ankle-

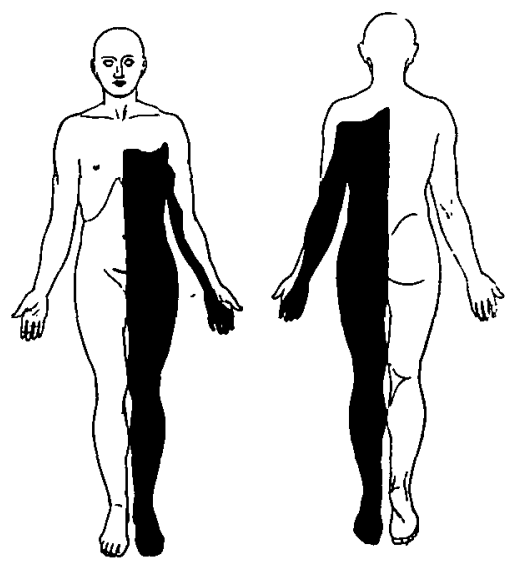

Fig. 41.

To show the loss of sensation to painful and thermal stimuli in Case 2.

clonus was easily obtained from the right foot, and the right toe gave a characteristic extensor response on stimulation of the sole. The right wrist-jerk was considerably in excess of the left. The left knee-jerk was diminished. No ankle-clonus could be obtained on this side, and the plantar reflex gave a definite flexor response.

Sensation.-He complained of a sensation of burning in the buttock down the left leg, principally on its outer side. A similar sensation troubled him in the left arm, mainly situated in the back of the hand.

Over the left half of the body, below the line marked in Fig. 41, all forms of painful stimulation and all degrees of temperature failed to produce any sensation. The border of this abnormal 
area seemed to be well-defined on the arm, but towards the periphery of the limb faded into parts of normal sensibility.

The lightest touch with cotton wool produced a sensation in every part of the body, and to this stimulus there was no difference between its two halves. Pressure was everywhere appreciated equally.

Cutaneous localisation was excellent to both light touch and deep pressure, and no difference could be discovered in the accuracy of localisation on the two sides of the body.

He was able to recognise the position into which his limbs had been moved passively, and his answers were quick and accurate, even for movements of the great toes.

The vibrations of the tuning-fork were appreciated everywhere.

Over the left half of the body and left leg, the strongest interrupted current produced no pain; but he said that a strong current gave him exactly the same sensation as that produced by holding a hot plate.

Sphincters. - Micturition and defæcation had never been affected in any way. At first, he was impotent without the power of erection, but in May had regained his power, and emission occurred, though more slowly than usual.

Cranial nerves.-The pupils were equal and reacted normally, dilating well to shade. The optic disc showed no hyperæmia and the fundus was normal. The face and tongue were not affected.

Spine.-The spine of the second cervical vertebræ bad sunk in consequence of collapse of the third cervical, and a radiograph showed this condition distinctly.

\section{Subsequent Proyress.}

On June 6, 1905, he was seen by Sir Victor Horsley, who admitted him to the National Hospital, Queen Square.

On June 25 the laminæ of the second, third, fourth and fifth cervical vertebræ were freely exposed; the third neural arch was found to have been fractured and pushed downwards. There was, however, no evidence of pressure on the spinal cord. The third arch was removed and the probe was passed both upwards and downwards, without the discovery of further fracture or pressure on the cord. But, in order to make certain, the laminæ of the fourth vertebræ were cut through. The cord was. seen to be pulsating well and there was no sign of hæmorrhage within the membranes. The wound was then closed. The patient stood the operation unusually well and showed little evidence of shock.

He recovered perfectly from the operation and the wound 
healed by first intention. For the first fortuight he was completely free from pain or abnormal sensations, but, at the end of that time, the burning in the left side returned worse than before. At the same time be noticed that the right arm seemed stiff and cramped.

In Oetober, 1905, he was again seen by one of us. He complained that " his right leg felt exactly as if it was a cork leg," and that all the muscles on this side "felt tight." He believed that he had lost power both in the right arm and leg since the operation.

Motion.-He still walked with a hemiplegic gait, and there was no obvious increase in the loss of muscular power. But all movements in the right leg and right arm had become more difficult in consequence of definite ataxy. He could no longer touch the tip of his nose with his right forefinger, nor bring the two forefingers together when the eyes were closed. He could not stand on his right foot with any certainty, and on closing his eyes fell at once.

There was no further wasting beyond that noticed in May.

Sensation.-O Over the left half of the body and left leg all sensation of pain, of heat, and of cold was abolished as before. (Fig. 41.) Light touch and pressure were perfectly appreciated and localised with certainty. The sense of passive position and movement was excellent, and the compass-test gave normal results; the vibration of the tuning-fork could be everywhere recognised.

Over the right leg, the right half of the body and the right arm, cutaneous painful stimuli were normally appreciated. Sensations of heat and cold were perfect. Sensibility to light touch and pressure was equal to that on the opposite side; but a remarkable change had occurred in the pain produced by increasing this stimulus. All down the right side and in the right leg, pressure with the head of a pin produced a painful sensation. There was no tenderness when the point was dragged gently across the skin, or when it was picked up between the fingers. But, as soon as the head of a pin was lightly pressed anywhere over the right half of the body, be complained of pain. Deep tenderness was certainly present.

Moreover, he was no longer conscious of the position into which his thumb, index and middle fingers of his right hand were placed passively. He also had considerable difficulty in recognising the position of his wrist. No passive movement of the toes of the right foot could be recognised, and his answers were frequently inaccurate after movements of the right ankle. None of the other physical signs were in any way altered. 
Shortly afterwards he left England, and we have had no furtber opportunity of examining him.

\section{Case 3.-Grace G.}

Sudden loss of power in the right leg on February 18, 1898, whilst sleeping.

The condition of motion and sensation has shown no material change since August, 1898, when she first came under our obser. vation.

The right leg is spastic and paretic. The right knee-jerk is much exaggerated, ankle-clonus is obtained on the right side and the plantar reflex gives an extensor response. On the left side the reflexes are normal, no ankle-clonus is present and the plantar reflex gives a flexor response.

Complete loss of sensation to pain and temperature over the left lower extremity (Fig. 42).

All forms of tactile sensibility are perfect.

Sense of passive position and movement and tactile discrimination (compass-test) are disturbed in the right foot.

No other abnormal signs are present in the nervous system.

Grace G., a married woman, aged 39, lay down to sleep as usual at 2 p.m. on the afternoon of February 18, 1898; she was then in perfect health. On rising an hour later, she was seized with pains round the body at the level of the umbilicus. She cried out for help and was found on her feet, holding the edge of the bed. She did not fall, but, when helped into bed, discovered she had lost all power in the right leg. For the first seven days she suffered with pain down the outer side of the right leg, which remained absolutely paralysed for three weeks. At no time was there any loss of power in the left leg.

From the first, she was able to recognise when the paralysed right leg was pricked, but it was not until four weeks after the onset that she discovered, on taking a bath, she was unable to discriminate heat from cold when applied to the left leg. The paralysed limb has remained throughout sensitive to these stimuli.

The complete paralysis of the right leg passed away gradually within the first two months; the leg became stiff and has remained in the same condition until the present time. For eight years the sensory condition of the left leg has remained unaltered. 
The date of the attack coincided with the first day of a normal menstrual period. She is usually regular at intervals of twentyeight days; the menstrual flow lasts for five days and is not usually profuse. Occasionally, however, since her marriage, she has gone two to three weeks over her normal time, and then the flow has been more profuse than usual. Five months after her marriage, she had an attack of what was thought to be peritonitis; she suffered much pain and was in bed for three weeks. During this time there was a constant discharge of blood from the vagina. This was possibly a miscarriage; but at no time has she ever been certain that she was pregnant.

There is no history of venereal disease and nothing pointing to the probability of infection.

She first came under the observation of one of us in August, 1898, five months after the onset of the paralysis. During the last eight years, she has been repeatedly examined; and, although we have amplified the scope of our observations, her condition has remained unchanged. The observations made in 1898 are in complete accord with those of the present time.

\section{Present Condition.}

She is a tall, well-built woman, of unusual intelligence, not anæmic nor wasted, and, except for the difficulty in walking, she says she is in perfect health.

Motion.-She walks with a stick, dragging the right leg, which she holds stiffly. She says she dare not let the right knee go slack, or she would fall. This leg is rigid and spastic; but the rigidity can be overcome by the exercise of steady pressure. At night she suffers from involuntary " jumping" of this leg. The right foot is in a position of slight pes cavus, due apparently to the extreme extensor response to all stimulation of the sole.

It is difficult to test the amount of voluntary power in this leg on account of its spasticity. But both dorsiflexion and plantar extension are feebly performed at the ankle-joint and flexion of the knee and hip is weak. Extension of the knee and hip can be more perfectly carried out, though less strongly, than with the left leg.

There is no profound wasting of either leg, but the right calf is one inch $(2.5 \mathrm{~cm}$.) smaller than the left at its largest circumference.

The motor power of the left leg is unaltered, and the upper extremities are entirely unaffected.

The electrical reactions, even of the right leg, are everywhere normal. 
Reflexes.-The right knee-jerk is greatly exaggerated and ankle-clonus is easily obtained. The right great toe gives an extreme extensor response when the sole of the foot is stimulated.

On the left side the knee-jerk is brisk, but there is no ankleclonus. The great toe either does not respond or occasionally gives a faint flexor response.

Sensation.-She complains that the right leg nearly always seems to her to be cold; but the left leg is never hot nor is it
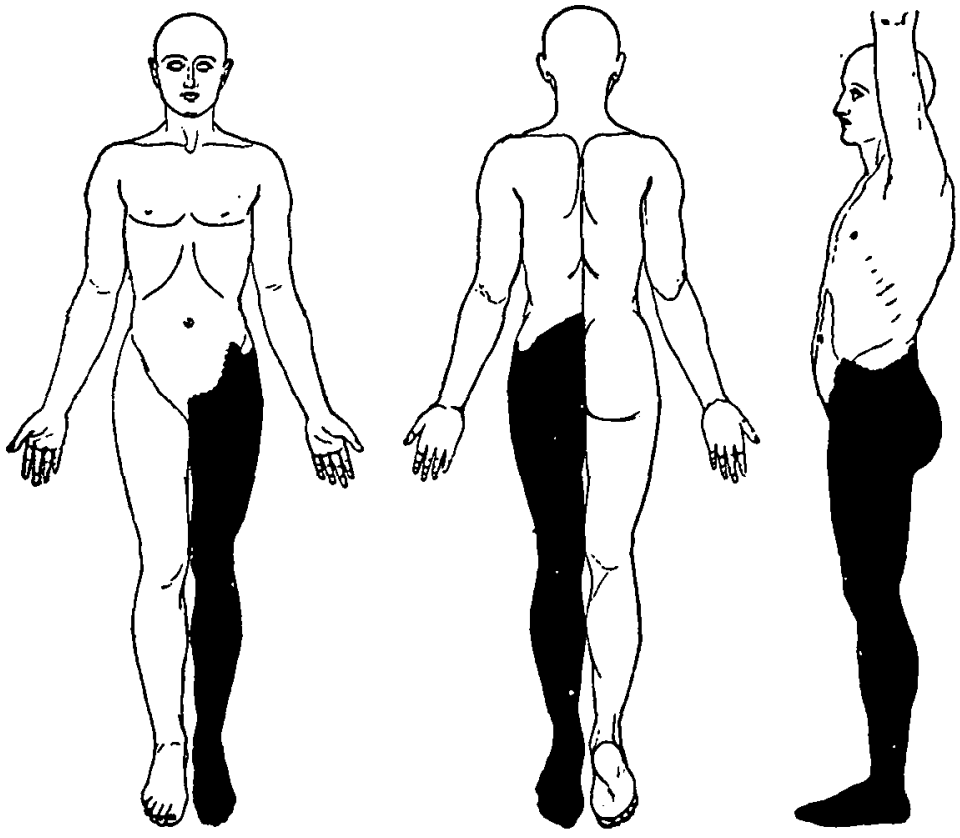

Fig. 42.

To show the loss of sensibility to painful and thermal stimulation in Case 3.

The border between the normal parts and those which were completely insensitive was unusually well defined, corresponding to the caudal border of the eleventh thoracic segment.

cold even in the winter. When she touches the right leg it always appears to be colder than the left. But, apart from these symptoms, she is not troubled by any pain or other abnormal sensation.

(a) Loss of Painful Sensibility.-The prick of a pin caused no pain over the whole of the left leg and thigh as high as the 
level of the iliac crest. The boundary between sensitive and insensitive parts was clearly defined, following the line marked upon Fig. 42. So sharp was this border that it made little or no difference whether the observations were made from the direction of normal to abnormal skin or vice vers $\hat{a}$.

The algometer gave the following readings, showing that the parts insensitive to a prick were also insensitive to the pain of pressure.

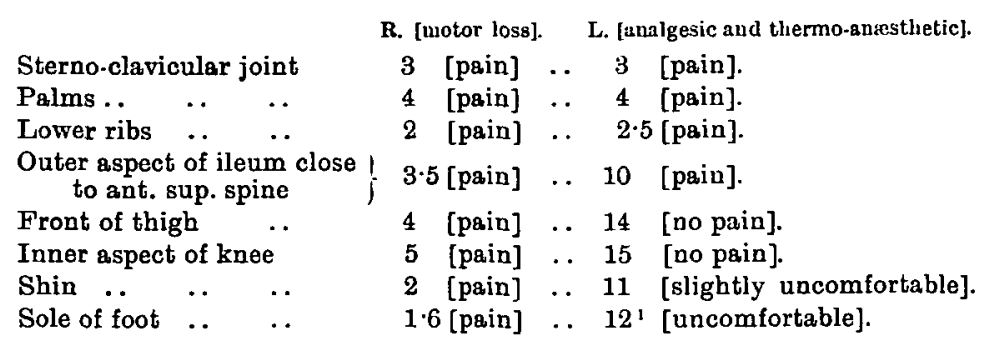

The loss of sensation to the painful interrupted current corresponded exactly to the area insensitive to prick. But she complains that when the current is applied to the left leg, which is completely analgesic, an exaggerated tickling sensation is produced. This is disagreeable and is associated with rapid withdrawal of the leg.

(b) Heat and cold.-Over the whole of the area on the left leg, shown in Fig. 42, she was entirely insensitive to all degrees of temperature. Over the right leg all temperature stimuli were correctly appreciated.

(c) Tactile sensibility.-Stimulation with cotton-wool produced a normal sensation, which was in no way different in the two legs. Pressure was everywhere correctly recognised, and even over parts where the algometer produced no pain the increasing pressure was distinctly appreciated. When tested with von Frey's hairs, no difference could be discovered between the two legs, but she was somewhat insensitive to this form of stimulation over both lower extremities. No. 5 [360 mgm.] was as frequently appreciated over the left as over the right leg; but over both legs she not infrequently failed to recognise this stimulus.

(e) Acucesthesia and appreciation of differences in șize.-Over

'She complains of an uncomfortable tickling when the algometer is pressed on to the sole of the left foot, which greatly disturbs the readings. Sometimes this condition makes it impossible to test the sole of the left foot. 
the whole of the analgesic leg she was able to tell the head from the point of a pin; in twenty stimulations to the sole of each foot, ten of which were made with the head and ten with the point, she did not give a single incorrect answer. Her power of recognising the relative size of objects was tested with three metal cylinders, $2 \mathrm{~cm}$., $75 \mathrm{~cm}$, and $\cdot 2 \mathrm{~cm}$. in diameter. In spite of the most varying combinations, her answers were always correct over both legs.

(f) Tactile localisation.-Light touches and deep pressure were localised with remarkable accuracy, whatever method we adopted. Sometimes she was asked to name the point touched, at others she was allowed to point to the spot with a cardboardrod. At other times, we adopted the method of touching the skin through a hole in a piece of paper, held a short distance above the skin, and she attempted to mark the spot touched with a soft pencil. At no time were we able to find the least difference between the two legs, and, when allowed to point, her accuracy and quickness was remarkable, although her eyes remained closed.

(g) Recoynition of passive position.-At every joint in the left lower extremity (analgesic and thermo-anæsthetic) her answers were remarkably quick and accurate. The position into which the right knee and ankle had been placed was also well recognised, but she was greatly at fault with regard to the position of the great toe on the right side. A comparison of the records of twenty stimuli in each foot, arranged graphically, shows this difference in a very striking manner, even the answers that were correct in the right foot were more slowly given than on the left side.

$$
\begin{aligned}
& \text { L. [analgesic and thermo-an:esthetic]. }
\end{aligned}
$$

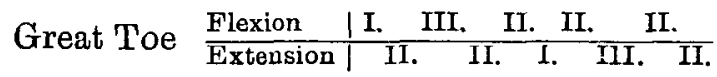$$
\text { R. [spastic]. }
$$$$
\text { Great Toe } \frac{\text { Flexion I II. }}{\text { Extension I XX. }} \text { I. }{ }_{\text {XI. }}^{\text {IIIX. }} \frac{\text { III. }}{\mathrm{XXIXX} .}
$$

L.

Ankle $\frac{\text { Flexion }}{\text { Extension | I. II. III. II. I. III. III. }}$

R.

Ankle $\frac{\text { Flexion | III. I. I. III. II. }}{\text { Extension | I. III. II. III. I. }}$ 
(h) Compass-test.-No difference between the two legs could be discovered until the foot was reached. Even over the feet the records, as she lay in bed, were almost equally good on the two sides.
L. [analgesic and thermo-anæsthetic]
R. [spastic].

Sole of foot . $2 \mathrm{~cm} . \quad \frac{1 \mid 7 R .3 \mathrm{~W} .}{2 \mid 10 \mathrm{R} .} \quad 2 \mathrm{~cm} . \quad \frac{1 \mid 9 R .1 \mathrm{~W}}{2 \mid 2 \mathrm{R} .8 \mathrm{~W}}$ $2.5 \mathrm{~cm} \cdot \frac{1 \mid 9 \mathrm{R} .1 \mathrm{~W} .}{2 \mid 10 \mathrm{R} .} \quad 2.5 \mathrm{~cm} \cdot \frac{1 \mid 8 \mathrm{R} .2 \mathrm{~W}}{2 \mid 8 \mathrm{R} .2 \mathrm{~W} .}$ $3 \mathrm{~cm} . \quad \frac{1 \mid 10 \mathrm{R} .}{2 \mid 10 \mathrm{R} .} \quad 3 \mathrm{~cm} . \frac{1 \mid 10 \mathrm{R}}{2 \mid 10 \mathrm{R} .}$

Dorsum of foot $2.5 \mathrm{~cm} . \frac{1 \mid 7 \mathrm{R} .3 \mathrm{~W} .}{2 \mid 10 \mathrm{R} .} \quad 2.5 \mathrm{~cm} . \frac{1 \mid 7 \mathrm{R} .3 \mathrm{~W}}{2 \mid 5 \mathrm{R} .5 \mathrm{~W}}$. 3 cm. $\frac{1 \mid 7 \text { R. } 3 \text { W. }}{2 \mid 10 \text { R. }} \quad 3$ cm. $\frac{1 \mid 7 \text { R. } 3 W}{2 \mid 9 \text { R. } 1 \text { W. }}$

Thus after a rest of two hours in bed there was no material difference between the formulæ at $3 \mathrm{~cm}$.

She then rose and walked 150 metres up and down a corridor. After returning to bed the difference between the two feet were distinct even at $4 \mathrm{~cm}$.

$$
4 \text { cm. } \frac{1 \mid 10 R .}{2 \mid 10 R .} \quad 4 \mathrm{~cm} . \quad \frac{1 \mid 6 \mathrm{R} .4 \mathrm{~W}}{2 \mid 6 \mathrm{R} .4 \mathrm{~W}}
$$

Thus, the only interference with her power of discriminating two points occurred over the right foot, i.e., on the side of the motor paralysis and on the side opposed to the analgesia.

(i) Tuning-fork.-The vibrations of a tuning-fork were everywhere recognised, and there was no difference between the two legs.

Sphincters.-She has no trouble in holding or passing her urine or fæces.

Cranial nerves.-The pupils react well : disc and fundus are normal. Hearing, smell and taste are perfect.

No other part of the nervous system is in any way affected.

There is no disease of the heart or lungs. The pulse is good, and the vessels show no abnormality. 


\section{Case 4.-Albert H.}

Fell into the hold of a ship on December 3, 1901.

Is said at first to have been able to move his right leg only, but when first seen by Mr. Sherren and one of us in February, 1902, the loss of power was confined to the left arm and leg.

$\mathrm{He}$ is a characteristic instance of Brown-Séquard paralysis.

Right.

No motor disturbance.

Knee-jerks normal.

No ankle clonus.

Plantar reflex flexor in type.

Loss of sensation to painful and thermal stimuli over an area shown in Fig. 43.

Diminution in tactile sensibility over the right foot, with coincident uncertainty in answers to the compass-test.

All other forms of sensation perfect.
Left.

Paresis and wasting of left hand.

Paralysis of Triceps.

Spasticity of left leg.

Knee-jerks greatly exaggerated.

Plantar reflex extensor in type.

Sensation perfect.

Albert $H$., aged 25, a boiler maker, fell into the hold of a ship on December 3, 1901.

He went below to screw up some frames but did not trouble to take a light. Expecting to step on to the lower deck, he walked into space, and was picked up head downwards unconscious in the hold.

He was taken to Poplar Hospital, where he lay unconscious until the evening.

When he woke be found he could move the right leg only.

In about three weeks he began to recover movement in the right arm. Gradually power came back in the left leg, so that by the end of January, 1902, he could walk; and when seen by Mr. Sherren and one of us in February, 1902, he said power was returning to his left arm.

It is impossible to make any statement with regard to the condition of sensation after the accident, as he confuses sensory with motor paralysis. The dominant facts in his mind concern the paralysis, first of both arms and the left leg, later of the left arm and left leg, and finally the condition which be calls paralysis of the left arm only. 
When be first came under the notice of Mr. Sherren and one of us in February, 1902, two months after the accident, his condition was as follows:-

He walked like a hemiplegic, dragging the left foot. He could not grasp with the left hand, and the movements of the left wrist were weak. The intrinsic muscles of the left hand were wasted. Dorsiflexion and plantar extension of the left ankle were feeble, and the left leg was spastic.

The left knee-jerk was greatly exaggerated; ankle clonus was obtained on the left side, and the left plantar reflex gave an extensor response. The wrist-jerk and elbow-jerk were exaggerated on the left side. All the reflexes in the right arm and leg were normal.

The sphincters were not affected, and there was no alteration in the reaction of the pupils to light or to shade. The left palpebral fissure was smaller and the left ear colder than on the right side.

The whole of the right half of the body below the line on Fig. 43 was insensitive to pain, heat, and cold; as the condition of sensation has changed little in the last five years, we shall give the further details later.

By July, 1902, he had considerably improved, and could walk without a stick.

Motor power steadily increased until the end of 1902 . Since that time his condition has remained stationary. He now keeps a small sweet-shop, and feels in perfect health except for the difficulty in walking.

During the last five years he has been examined repeatedly, and the following account, based on his condition during the latter half of 1906, includes some of the observations made throughout the previous period.

\section{Present Condition.}

He is a short, somewhat youthful-looking man of moderate intelligence. He has never been intemperate, and does not now take any alcohol.

Except for the defects in the nervous system he shows no symptoms or signs of disease.

Motion.-He walks like a man with hemiplegia, sweeping the left leg outwards to enable the left foot to clear the ground. At the same time the left leg is kept rigidly extended. He can stand steadily with the eyes open or shut, but has difficulty in standing on either leg alone. 
The left leg is distinctly spastic. He can slightly extend the toes but cannot dorsiflex the ankle; flexion of the toes and plantar extension of the ankle are slowly and feebly performed against resistance. If given time he can flex and extend the knee; but the movement is feeble against resistence. Movements at the hip are better performed, and, though slow, are powerful.

The left leg is not wasted.

The left hand is held with the wrist a little flexed and the fingers doubled into the palm. The fingers cannot be extended unless the wrist is fully flexed, in consequence of the shortening of the flexor muscles. He seems to have lost the power of straightening the fingers voluntarily, but can grasp feebly. $\mathrm{He}$

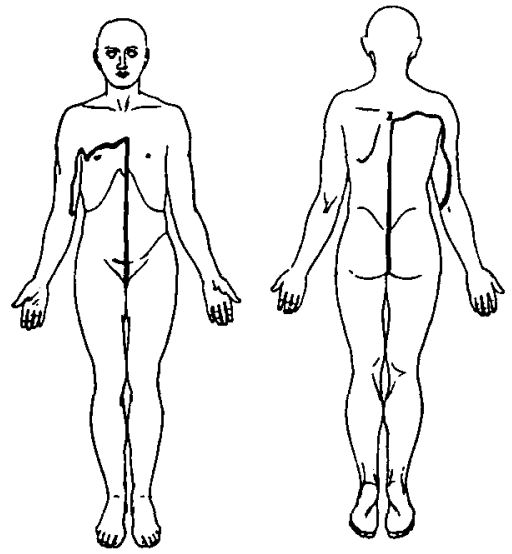

FIG. 43.

To show the border at which sensation to pain, heat and cold ceased in Case 4 .

can extend the wrist well until this movement is checked by the shortening of the flexor muscles, and can pronate and supinate the forearm.

The triceps acts extremely badly, if at all; when the arm is flexed and the patient is told to extend it he moves the whole arm until it is jerked into the extended position. The biceps, deltoid and supinator longus contract well, and the muscles of the left shoulder are unaffected.

The interosseous spaces of the left hand are hollowed and all its small muscles are somewhat wasted. The left forearm is $1 \mathrm{~cm}$. and the left arm $1.5 \mathrm{~cm}$. smaller in circumference than the right.

All the muscles of the left upper extremity, with the exception of the triceps, react normally to electrical stimulation. The triceps 
reacts badly, if at all, to the strongest interrupted current, and, on closing the constant current, contracts more easily to the anode than to the kathode.

On the right half of the body every motor function has been perfectly normal since he first came under observation, three months after the accident.

Reflexes.-The left knee-jerk is greatly increased; ankle clonus is obtained on the left side, and the plantar reflex gives an extensor response.

On the right side the knee-jerk is normal, no ankle clonus can be obtained and the plentar reflex is of the flexor type.

Wrist and elbow jerks are equal on the two sides.

Sensation.-He complains of no abnormal sensation and says that if he had not been tested he would not have known that sensation was in any way defective.

(a) Sensibility to painful stimuli.-At the line marked on Fig. 43 a profound change occurs in his sensibility to painful cutaneous stimuli, such as a prick or the painful interrupted current. All sensibility to pain is lost as soon as this line is passed, when travelling from normal to abnormal parts; at the same time, when passing from the analgesic area he becomes sensitive at this line. Throughout the five years he has been under observation this border has always been well defined, and Fig. 43 exactly corre. sponds with a photograph taken in November, 1902.

$\mathrm{He}$ is insensitive to the pain of deep pressure over the right half of the body as high as the lower ribs, and the following readings in kilograms were obtained by means of the pressure algometer.

\begin{tabular}{|c|c|c|c|c|c|c|c|c|c|c|c|}
\hline & & & & & & & & R. & & & L. \\
\hline Sole & . & .. & . & . & . & . & 15 & (no pain) & . & 4 & (pain). \\
\hline Internal & malleo & olus & . & .. & . & .. & 12 & (no pain) & . & 3.5 & (pain). \\
\hline Shin & .. & .. & $\cdots$ & $\ldots$ & $\ldots$ & $\ldots$ & 14 & (no pain) & $\ldots$ & $3 \cdot 5$ & (pain). \\
\hline Inner asp & pect of & knee & $\ldots$ & $\ldots$ & $\ldots$ & $\ldots$ & 14 & (no pain) & $\ldots$ & 5 & (pain). \\
\hline Front of & thigh & . & $\ldots$ & . & . & $\ldots$ & 13 & (no pain) & .. & 5 & (pain). \\
\hline Outer par & rt of ar & nt. sup & spi & of & & .. & 12 & (no pain) & $\ldots$ & 3 & (pain). \\
\hline Lower rib & & . & $\ldots$ & . & . & . & 4 & (pain) & $\ldots$ & 2 & (pain). \\
\hline Palm & .. & .. & .. & . & .. & . & 7 & (pain) & $\ldots$ & 6 & (pain). \\
\hline Shoulder & .. & . & .. & $\ldots$ & .. & . & 4 & (pain) & . & 4 & (pain). \\
\hline Clavicle & $\ldots$ & $\ldots$ & . & .. & . & . & 2 & (pain) & . & 2 & (pain). \\
\hline Mastoid & .. & $\ldots$ & $\ldots$ & .. & $\ldots$ & $\ldots$ & 2 & (pain) & $\ldots$ & 2 & (pain). \\
\hline Temple & . & . & . & . & $\ldots$ & . & & 5 (pain) & $\ldots$ & 1.5 & (pain). \\
\hline
\end{tabular}

He was able to recognise the gradually increasing pressure everywhere over the analgesic area, although it caused no pain.

(b) Sensibility to thermal stimuli.-Throughout the five years, voL. XXIx. 
he has been entirely insensitive to all thermal stimuli over the right leg and right half of the body below the line on Fig. 43.

He has been carefully tested on many occasions with temperatures of $0^{\circ} \mathrm{C} ., 20^{\circ} \mathrm{C} ., 30^{\circ} \mathrm{C} ., 40^{\circ} \mathrm{C}$, and $50^{\circ} \mathrm{C}$, and found to be insensitive to all degrees. The patch on the inner aspect of the right arm can be perfectly defined with thermal stimuli.

(c) Tactile sensibility.-Touches with cotton wool can be appreciated everywhere; but over the sole of the right foot his answers are less certain to this stimulus than over the opposite sole. This difference becomes measurable when be is tested with von Frey's hairs. For over the left sole and left shin he recognised No. 8 ( $830 \mathrm{mgm}$.) with ease, whilst over similar parts on the right lower extremity this stimulus produced no sensation. This diminished tactile sensibility appears in the defective compassrecords obtained over the right sole.

Sensibility to deep pressure was nowhere appreciably different on the two sides of the body.

(d) Compass-test.-The records obtained to this test were everywhere perfect and equal on the two sides except those obtained from the right sole. Here his answers were slower and somewhat less perfect, and he recognises that this is due to a diminution of tactile sensibility. He says be cannot "feel the touch so well "over the right foot. We believe that this is an instance where the compass-records are disturbed by defective tactile sensibility rather than by a loss of tactile discrimination.

Over the palm his answers are equally quick and accurate at $1.5 \mathrm{~cm}$. But over the soles we obtained the following results.

Compasses applied transversely :-

\section{R.}

$2 \mathrm{~cm} \cdot \frac{1 \mid 10 \mathrm{R} .}{2 \mid 10 \mathrm{~W}}$.

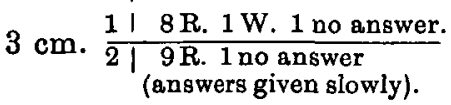

\section{L.} \begin{tabular}{l|l|l}
1 & 9 R. $1 \mathrm{~W}$ \\
\hline $2 \mid 9$ R. 1W.
\end{tabular}

$\frac{1 \mid 10 R}{2 \mid 10 R}$.

(answers quick).

At $4 \mathrm{~cm}$. his answers were equally good over both soles.

When the right sole is tested the accuracy of his answers can be greatly improved by practice. Thus he may fail on four or five occasions to answer at all during a series of twenty stimuli with one or two points separated at a distance of $3 \mathrm{~cm}$. But after further testing of the sole with the points at different distances he sometimes gives a perfect series of answers at $3 \mathrm{~cm}$.

(e) Passive position and movement can be perfectly appreciated on both sides of the body, and there is no difference in the records 
obtained from the great toes, ankles, knees, hips, or any joints of the upper extremities.

( $f$ ) Tactile localisation is equally good on the two halves of the body both to naming and groping.

(g) The vibrations of a tuning-fork were equally well appreciated everywhere over the two halves of the body.

(h) Appreciation of differences in size.-Cylindrical rods were used with diameters of $2 \mathrm{~cm} ., 0.6 \mathrm{~cm}$., and $0.2 \mathrm{~cm}$. The difference in size could be appreciated equally well over the two halves of the body except the soles, which could not be tested with certainty.

Cranial nerves.-Taste and smell are unaffected. There has been no limitation of the visual field in either eye, and disc and fundus have always been normal.

The pupils are equal and react normaily to light and to shade. The left pupil dilates well to cocaine.

The movements of the face and tongue are normal.

Sphincters. - Micturition and defæcation have never been affected in any way.

Spine.-No gross abnormality is visible in the spine, but a radiograph shows that the fourth neural arch has been fractured.

\section{Case 5.-Walter M.}

May 18, 1904, he fell 15 feet on to a heap of bricks. Immediate loss of power in the right arm and leg. Shortly afterwards defective sensation was discovered in the left leg.

Seen by us in 1906.

He was then a characteristic instance of Brown-Séquard paralysis.

Right Side.

Wasting of muscles of hand. Spasticity of wrist and of leg. Knee-jerks increased. Ankle-clonus.

Extensor plantar reflex. No change in sensation.
Left Side.

No paralysis or muscular wasting.

Reflexes normal.

Loss of sensibility to pain, heat and cold.

Tactile sensibility, tactile localisation, tactile discrimination, sense of passive position and movement unaffected.

Walter M., aged 46, a carpenter and joiner, fell $15 \mathrm{ft}$. on May 18, 1904. He was working on a scaffold, and, in attempting 
to cross an opening, supported himself by a wooden rail ; this broke, and he fell on to a rough heap of bricks. He did not lose consciousness, but was carried by his fellow workmen to St. George's Hospital, where he lay three or four weeks.

He was much bruised, especially at the " bottom of the spine." The right arm was weak and, to a less extent, he had also lost power in the right leg; but at no time was he unable to move both the leg and the arm. He was told by the house physician that over the left leg he did not answer correctly to the prick of a pin, and to hot and cold tests. This surprised him, for he was not conscious of any defective sensation in this leg.

On leaving the hospital, he was able to walk unsteadily. Gradually his gait improved, and he could do a little work, but his neck seemed to want support. $\mathrm{He}$ suffered no pain, but when he sat up or walked for a time, he felt an uncomfortable sensation in the region of the seventh cervical spine, which occa. sionally became an ache.

He has had no abnormal sensations in the left leg, but when he takes a hot bath he always tests the water first with his right foot lest he should burn bimself. When he dips the left leg into cold water, it produces numbness and not a sensation of cold.

He thinks the strength of the right arm and leg is still improving.

He has never had trouble with micturition or defæcation. Since the accident, he has had no connection with his wife, and, although occasionally he may have an erection, there has been no emission. Sexual desire is evidently slight.

He has been married for nineteen years and his wife has had no miscarriages. No history could be obtained of venereal disease. He is a life-long teetotaler and non-smoker.

Condition in October, 1906.

He was an unusually intelligent man, self-educated to a high degree. Somewhat excitable in manner and speech, he was abso. lutely trustworthy to control-tests.

Motion.-He walked well, holding the right leg a little stiffy with a slight drag of the right foot. He could stand equally well with his eyes open or shut. The right leg was distinctly spastic, and he complained that it "jumped" at night occasionally. Neither leg showed any signs of wasting, and the measurements were equal on the two sides.

The left hand was in every way normal, but the interossei and hypothenar muscles of the right hand were distinctly wasted, 
and in them could be seen fibrillary twitchings. Not only were these muscles wasted, but the fingers were rigid; the proximal phalanges were hyper-extended, and the two distal phalanges slightly flexed. The thenar muscles were in a similar condition, much wasted compared with those of the other hand, and the thumb was adducted and flexed in consequence of the spasticity. Every movement of the right thumb could be carried out, though feebly. The grasp of the right hand was 2 kilograms, that of the left 25 kilograms. All the small muscles of the right hand were less excitable to the interrupted current than those of the left; to the constant current they contracted sluggishly, and more easily on closure to the anode than to the kathode.

All movements of the right wrist could be carried out, but were distinctly spastic. Movements of the elbow were normal and there was no spasticity.

The muscles of the post-axial balf of the right forearm were somewhat wasted; the difference between the two forearms at a point $20 \mathrm{~cm}$. above the lower end of the radius was $1.5 \mathrm{~cm}$. (right $22 \mathrm{~cm}$., left $23.5 \mathrm{~cm}$.).

All the muscles of the right forearm and arm reacted well to electrical stimulation.

On quiet respiration the right half of the chest moved distinctly less than the left; this difference became more evident when the patient breathed deeply. Similarly, the movement of the left was greater than that of the right half of the abdomen; when told to contract the muscles of his abdomen, the mid-ven. tral line was drawn over to the left.

Reflexes.-On the right side, the knee-jerk was greater than that on the left, and ankle-clonus was obtained. The right plantar reflex was invariably of the extensor type, whilst flexion was always obtained from the left foot. The right elbow-jerk was definitely brisker than that of the left side.

Sensation.- (a) Sensibility to pain.- - He was entirely insensitive to the prick of a pin over the whole of the left half of the body as high as the level of the third rib, and over a small area on the inner aspect of the left arm (Fig. 44). The limits of this area were well defined in front and on the arm; they varied little according to whether the patient was tested from analgesic parts towards those of normal sensibility or vice versa. Over the scapula the border differed by about 2 to $3 \mathrm{~cm}$. according to the method of testing employed.

When tested with the painful interrupted current, the analgesia was found to agree exactly with that mapped out by means of the prick of a pin. 
But, although he was entirely insensitive to the pain produced by the current, it caused violent movements of withdrawal. He complained that the sensation, though in no way painful, was extremely uncomfortable.

Squeezing the right testicle between the fingers caused the usual sickening sensation; when the left testicle was squeezed he appreciated the pressure, but no pain or discomfort was produced.

The pain of pressure was not appreciated as high as the level of the seventh rib on the left side. The algometer gave the following readings :-
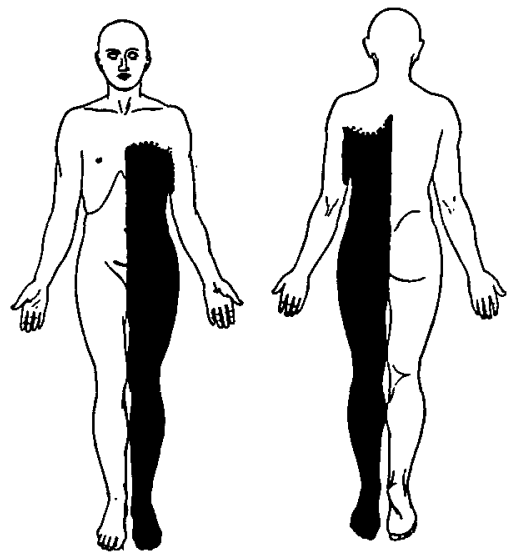

FIG. 44.

To show the loss of sensation to pain, heat and cold in Case 5 .

\begin{tabular}{|c|c|c|c|c|c|c|c|}
\hline Sole & .. & . & .. & & $\begin{array}{l}\text { R. (spastic). } \\
\text { Pain at } 4\end{array}$ & .. & $\begin{array}{l}\text { L. (analgeaic). } \\
\text { No pain at } 15 \text {. }\end{array}$ \\
\hline External malleolus & .. & . & . & . & Pain at 3 & .. & No pain at 10. \\
\hline Shin $\quad \ldots \quad \ldots$ & . & . & .. & & Pain at $4 \cdot 5$ & . & No pain at 13 . \\
\hline Outer aspect of knee & $e \ldots$ & $\ldots$ & $\ldots$ & .. & Pain at 5 & $\cdots$ & No pain at 12 . \\
\hline Front of thigh.. & .. & . & $\ldots$ & $\ldots$ & Pain at 5 & . & No pain at 15 . \\
\hline $\begin{array}{l}\text { Outer surface of } \\
\text { spine }\end{array}$ & anteriol & & erior & iliac & Pain at 3 & .. & No pain at 12 . \\
\hline Lower ribs .. & & . & . & . & Pain at $1 \cdot 5$ & . & No pain at 10 . \\
\hline Over seventh rib in & axilla & .. & $\ldots$ & & Pain at 1.5 & $\ldots$ & Slight pain at 7. \\
\hline Inner aspect of arm & $\ldots$ & . & . & . . & Pain at 1.5 & $\ldots$ & Pain at 7 \\
\hline Clavicle $\quad$.. & .. & . & .. & .. & Pain at 1.5 & . & Psin at 1.5 . \\
\hline Palm .. & .. & . & .. & .. & Pain at 6 & .. & Pain at 6. \\
\hline Elbow .. & . & . & . & & Pain at 5 & .. & Pain at 5. \\
\hline Shoulder & .. & $\cdots$ & . & $\cdots$ & Pain at 3 & $\cdots$ & Pain at 3. \\
\hline Temple & . & . & . & .. & Pain at 2 & . & Pain at 2. \\
\hline
\end{tabular}


(b) Thermal sensibility.-Any object brought into contact with the area on Fig. 44 was usually said to be warm, even if it were a tube containing ice. This made the testing of thermal sensibility extremely difficult. But, by varying the stimulus and by employing as a control a test-tube at a temperature which seemed neither hot nor cold to the normal parts, we were able to assure ourselves that neither heat nor cold were appreciated over the affected area. If a tube containing ice was passed slowly down the chest from the left clavicle, it was called "warm" as soon as it entered the area on Fig. 44 . Similarly, water at $25^{\circ}$ and at $45^{\circ}$ and the neutral test-tube seemed to him equally "warm."

(c) Sensibility to light touch.-Stimulation with cotton wool was quickly and accurately appreciated everywhere; no difference could be discovered between the two sides of the body.

Tested with von Frey's hairs we found that No. 5 (360 mgm.) could be appreciated on both palms and both soles. No. $8(830$ mgm.) produced a sensation everywhere on the abdomen, the front of the thighs and the back of both calves.

(d) Deep touch.-He recognised deep touches and was conscious of the gradual increase of the pressure caused by the algometer, even when it failed to produce pain.

(e) Acucesthesia.-The head of a pin could be correctly discriminated from the point everywhere over the analgesic area.

(f) Appreciation of difference in size.-Tested by cylindrical metal rods with diameters of $2 \mathrm{~cm}$., $0.75 \mathrm{~cm}$. and $0.2 \mathrm{~cm}$., he was equally accurate in his answers on both halves of the body.

(g) The vibrations of a tuning-fork (128) were accurately appreciated everywhere. There was no difference between the two halves of the body.

(h) The sense of passive position was perfect in both great toes, both ankles, both knees, and at all the joints of both upper limbs.

(i) The compass-test.-This was difficult to apply, owing to the violent reflex movements which were produced when the two points were brought into contact with the skin over any part of the analgesic and thermo-anæsthetic area, particularly over the sole of the left foot. This made it impossible to determine the threshold with any accuracy on the left side. But, when the points were applied carefully to the left sole and those answers only recorded that were unaccompanied by a violent reflex movement, we could not assure ourselves that there was any difference between the response from the right or the left foot.

(j) Tactile localisation was unusually accurate, and we could find no difference between the two sides of the body. 
Cranial nerves. - The pupils reacted well to light and to shade. The movements of the eyes, face and tongue were perfectly performed.

Spine.-A radiograph of the spine showed disorganisation of the sixth and seventh cervical vertebræ.

\section{Case 6.-James M.}

He injured his spine by diving into shallow water in June, 1886. He did not lose conscionsness at once, but was delirious for six weeks.

He gradually recovered power, and in three years reached his present condition. He came to the London Hospital in December, 1906, for lead colic, and was found to be a characteristic instance of Brown-Séquard paralysis.

\section{Right.}

No motor disturbance.

Reflexes normal.

Loss of sensation to pain, heat, and cold (Figs. 45 \& 46).

Tactile sensibility perfect.

\section{Left.}

Wasting of small muscles of hand.

Slight spasticity of leg.

Knee-jerk exaggerated.

Ankle-clonus.

Plantar reflex, extensor.

No change in sensation.

Passive position and movement perfectly appreciated. Compass-test gave perfect results.

James M., aged 41, a painter, came to the London Hospital in December, 1906, complaining of an attack of lead colic. He was discovered to bave the symptoms of Brown-Séquard paralysis, of which he gave the following history.

In June, 1886, he was in the Army and was stationed at Aden. It was the custom of those men who could swim to bathe in the evening from Steamer Point. So large were the waves that it was possible to dive into them with safety. But one evening in June when attempting this feat, he miscalculated his distance and, diving into shallow water, struck the sand with his head. All power went from his limbs immediately, but he did not lose consciousness even for a moment. He was swept back by the wave, but was brought to land and stretched out upon the sand. $\mathrm{He}$ 
suffered no pain, but seemed as if swollen to four times his usual size. They carried him to hospital and put him to bed; then be went quietly to sleep and did not regain consciousuess for six weeks. He was told that he was delirious all the time. When be came to himself he found that he had several bed-sores; he could move his hands a little but no other limb.

Slowly, power returned to the right leg and both arms. It was six months before he could stand and then the left leg dragged.

He was sent to Netley and remained there until June 26, 1887 , able to walk but dragging the left leg.

After his discharge from the Army he found work, and in three years had reached exactly the condition in which be first came under our notice.

$\dot{H} e$ is a foreman painter and is in charge of the mixing of the paint. Once or twice a year he has suffered from pains in the stomach, evidently slight attacks of lead colic; be comes to the hospital and rapidly recovers. It was on one of these visits that his condition was discovered by Mr. Sherren, who transferred him to us.

\section{Condition in December, 1906.}

He is an intelligent man, temperate in his habits and answers well to sensory tests. He thinks there is nothing amiss with him, but was content to lie still in bed under observation as often as he was wanted, provided arrangements were made for his men to continue their work.

Motion.-He could stand with his eyes open and shut, and was in no way ataxic.

When he walked, the left leg was held a little stiffly, and he said be could not run, "because the left leg would catch." So long as he walked slowly, the toe cleared the ground completely. All the movements of both lower extremities were strong against resistance and there was no paralysis of any group of muscles.

He said that the left leg occasionally "jumped" at night; this never happened to the right leg.

The small muscles of the left hand were definitely wasted; all the interosseous spaces were hollowed and the hypothenar eminence was unnaturally small. The outer group of thenar muscles, formed by the opponens and abductor, was well developed, but the remaining muscles of the thumb were wasted compared with those of the opposite hand. He could strongly abduct and oppose the thumb, and adduction and flexion were possible; extension was perfect. 
The grasp of the left hand was 2 kilograms, compared with a dynamometer reading of 95 kilograms from the right hand. This profound difference in the two hands was produced by the diffculty with which he grasped the instrument, in consequence of the want of power to flex the metacarpo-phalangeal joints strongly ; and yet, when he made this movement apart from the dynamometer, it was carried out perfectly. The loss of grasp, when he attempted to exert pressure by flexing the fingers into the palin, seemed to be due to weakness of the interossei. The fingers could not be held out straight without falling out of alignment, and the interossei and lumbricales were acting feebly, if at all. All the long
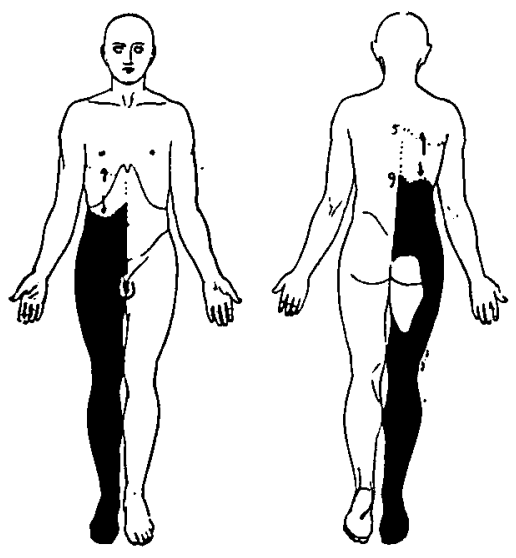

Fig. 45.

To show the loss of sensation to prick in Case 6 . The line marked with an arrow pointing upwards corresponds to the border of the cutaneous analgesia discovered by testing from insensitive to sensitive parts. The border marked with an arrow pointing downwards shows the limits of the sensibility to pain when he was tested from normal to insensitive parts.

flexors and extensors acted well, and there was no loss of power in the muscles of the elbow and shoulder.

The muscles of the right upper extremity reacted well to both currents. The only changes in electrical irritability were found in the small muscles of the left hand; the interossei and the abductor minimus digits did not respond to the interrupted or constant currents. The outer thenar muscles reacted briskly and normally, but the inner. group contracted poorly to both forms of electrical stimulation.

Reflexes.-Wrist- and elbow-jerks were equal on the two sides. But the left knee-jerk was much exaggerated. Ankle-clonus was 
obtained and the plantar reflex on this side gave an extensor response.

On the right side, the knee-jerk and plantar reflex were normal and no ankle-clonus could be obtained.

Sensation.-Except for the slight colic, which rapidly passed away, he complained of no abnormal sensations of any kind. But he found that, when the weather grew cold, he lost the use of his left hand, although in warm weather he could notice little difference between the two hands.

(a) Sensibility to pain.-To all cutaneous painful stimuli, such as the prick of a pin, sensation was lost over the area on the right half of the body shown in Fig. 45. The frontal boundary of this analgesia varied according to the method of testing. If the stimulus travelled from parts that were insensitive to those of normal sensibility, the analgesia extended up to the higher of the two lines; but, when the testing began over normal parts, he still responded to the painful stimulus, until the second of the two lines was passed. This is a characteristic feature of areas which are partly insensitive only.

Another remarkable feature of this case was the maintenance of sensation to painful cutaneous stimuli over a patch upon the right buttock and over the whole of the penis and scrotum.

The pain of excessive pressure was greatly diminished over the right sole and right leg below the knee. The algometer gave the following readings.

\begin{tabular}{|c|c|c|c|c|c|c|}
\hline Sole & . & . & . & . & $\begin{array}{c}\text { R. } \\
\text { Pain at } 14\end{array}$ & $\begin{array}{c}\text { L. } \\
\text { Pain at } 4 .\end{array}$ \\
\hline Inner aspect of leg & $\cdots$ & .. & .. & .. & Pain at 8 & Pain at 2.5 . \\
\hline Outer aspect of leg & $\cdots$ & .. & .. & .. & Pain at 15 & Pain at 7 \\
\hline Calf .. & . & .. & .. & .. & No pain at 12 & Pain at 8. \\
\hline Knee .. & .. & . & .. & . & No pain at 13 & Pain at 5. \\
\hline Front of thigh & . & .. & . . . & .. & Pain at 7 & Pain at 6. \\
\hline Ischial tuberosity & $\cdots$ & . & .. & .. & Pain at 8 & Pain at 5. \\
\hline Iliac crest .. & . & .. & .. & . & Pain at 4 & Pain at 4. \\
\hline Sterno-clavicular $j$ & oint & . & . & . & Pain at 2 & Pain at 2. \\
\hline
\end{tabular}

When a strong current was applied to the right or analgesic leg, he complained that it was " uncomfortable but not painful." Then the same current was applied quickly to the left calf and immediately withdrawn on account of the pain; but this stimulation had caused "cramp" in the left calf and he sat up in bed, rubbing his leg. Thus it is evident that the deep muscular pain upon which " cramp" depends must have been abolished in the right leg. This agrees with the results obtained with the algometer. 
(b) Thermal sensibility. - He was insensitive to all degrees of cold over the area shown on Fig. 46. The extent of anæsthesia to all degrees of heat almost exactly corresponded with that for cold.

(c) Tactile sensibility was perfectly preserved; cotton wool was everywhere recognised, and No. 5 (360 mgm.) of von Frey's hairs produced a sensation of equal intensity over the great toes and over the sole and dorsum of both feet.

(d) The vibrations of a tuning-fork (128) were perfectly appreciated everywhere.

(e) Acucsihesia. - He could tell the difference between the bead and the point of a pin everywhere. In fact he is so clever
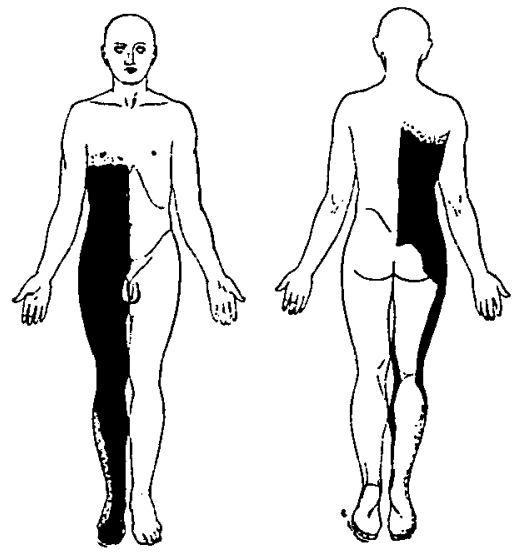

Fio. 46.

To show the extent of the loss of sensibility to thermal stimuli in Case 6 .

at telling the head from the point that it is sometimes difficult to work out the loss of painful cutaneous sensibility by means of a pin.

(f) Sense of passive position and movement was everywhere perfect. His answers in response to movements of either foot were unusually quick and certain. So trustworthy was he that throughout the testing of the great toes, ankles, knees, elbows, wrists, thumb, index and middle fingers, he did not make a single mistake, although 20 movements were made at each joint (320 stimulations).

(g) The Compass-test.-He gave perfect answers over the palms at $2 \mathrm{~cm}$. and over the forearms at $4 \mathrm{~cm}$. distance. When 
the compasses were applied longitudinally to the sole of the foot, his answers were perfect at $4 \mathrm{~cm}$., but were almost equally faulty at $3 \mathrm{~cm}$.

Right $3 \mathrm{~cm} \cdot \frac{1 \mid 9 \text { R. } 1 \mathrm{~W} .}{2 \mid 8 \mathrm{R} .2 \mathrm{~W} .} \quad$ Left $3 \mathrm{~cm} \cdot \frac{1 \mid 7 \mathrm{R} .3 \mathrm{~W} .}{2 \mid 9 \mathrm{R} .1 \mathrm{~W}}$

Thus, there was certainly no difference in the sensibility of the two soles to this test.

(h) Tactile localisation was equally good on the two halves of the body. When asked to name the spot touched, he did so correctly in every case, even when the touch was produced with No. 8 (830 mgm.) of von Frey's hairs.

Tested by Spearman's method (vide pp. 548 and 627), the results were as follows: Six touches were given at each spot and the numbers represent the average deviation of his six attempts to point to the spot stimulated :-

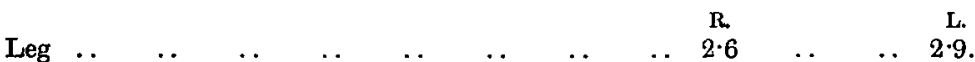

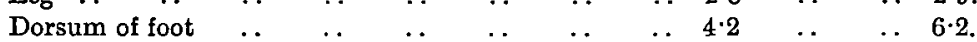

The difference between the two sides was, we believe, within the experimental error.

Cranial nerves.--The pupils reacted well, and there was no abnormality in the function of any cranial nerve.

Spine.-A radiograph showed no abnormality of any kind.

Sphincters.-He could hold his water for half an hour, but it occasionally dribbled away a little if he attempted to hold it too long. So long as he remained constipated, he could hold his motions; but when they became loose, he lost all control.

Sexual.-He has no desire during the winter months; sometimes during the summer he feels desire, but has never had an erection or emission since his accident. After leaving the Army, he married "for the sake of a home," and has no children.

\section{CASE 7.-Cyril Wilfred F.}

August 14, 1905, he dived into shallow water and injured his spine. Sensation was affected in the left leg, and, after the effects of shock had passed away, his right arm remained weak.

Seen in November, 1905, there was no definite motor paresis, but the reflexes were brisker on the right side than on the left. Sensibility to pain, to cold and to heat were lost to a different cxtent ovcr the left leg and left half of the body (Figs. 47, 48, \& 49). 
No other sensory function was disturbed. A radiograph showed changes in the body of the sixth cervical vertebra.

In 1906, observations were made on the behaviour of the area on the trunk insensitive to heat, but sensitive to cold, in order to elucidate the condition known as paradox cold.

Cyril Wilfred F., aged 26, of no occupation.

On August 14, 1905, whilst diving from the top of a bathing. machine, be slipped, pitching head first into shallow water. He believes that the top of his head struck the sand. He floated to the top, and did not lose consciousness, but found he was' unable to move his arms or his legs. Power returned in all his limbs within half an hour, but he remained in bed for three weeks,
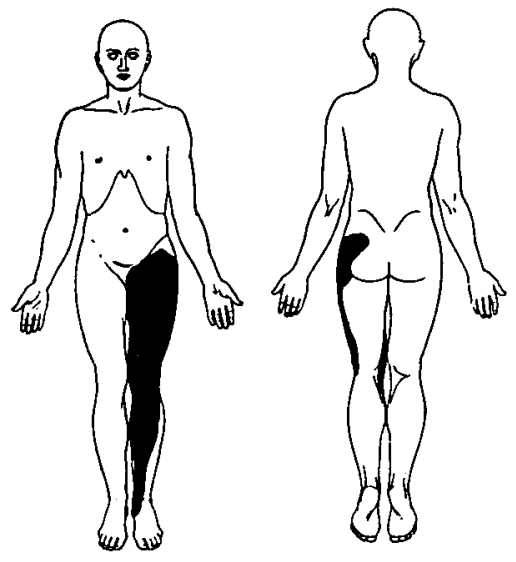

Fra. 47.

To show the loss of sensibility to cutaneous painful stimuli in Case 7 .

suffering from a good deal of pain in the neck. For nearly three months be could not use the right arm, and, from the beginning, noticed that sensation was affected in the left leg, particularly to heat and cold. At first the neck was stiff, and he suffered from pain, especially on movement, at a level of about the seventh cervical vertebra.

Condition in November, 1905.

He came under our notice in November, 1905, exactly three months after the accident. His condition then was as follows. 
He was a small, well-built, muscular man, and showed no signs of any disease, except in the nervous system.

Motion.-He walked well, and could stand perfectly with his eyes closed. There was no sign of paralysis, or spasticity in the legs. The grasp of the left hand was distinctly more powerful than that of the right, but there was no wasting or paralysis in either upper limb. He could touch his nose perfectly with either hand, even when his eyes were closed.

Reflexes.-The right wrist-jerk was slightly brisker than the left, and the knee-jerk was distinctly more active on the right side than on the left. No ankle-clonus was present on either side. When testing for the plantar reflex, the left great toe gave a decided flexor response, but the right remained stationary.
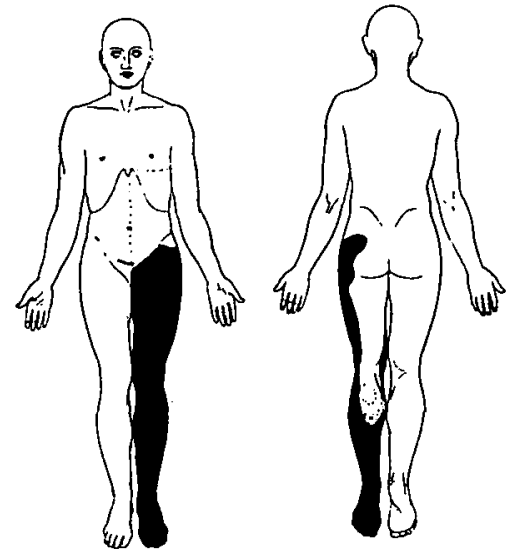

FIG. 48.
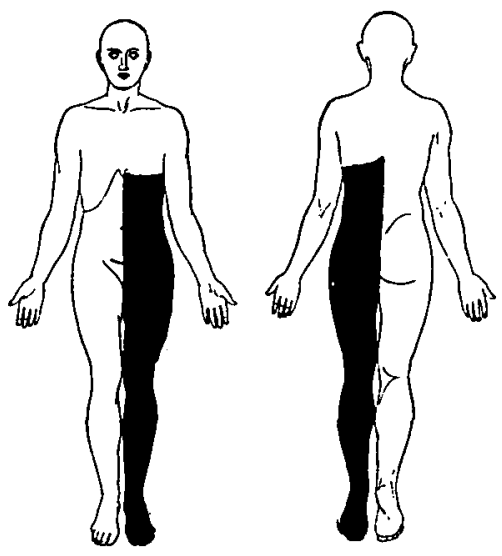

FIG. 49.

To show the loss of sensation to cold (Fig. 48) and to heat (Fig. 49) in Case 7.

The area on Fig. 48 enclosed by a dotted line was sensitive to cold, but not to heat. It was here that the observations were made on the production of paradox cold by temperatures of $45^{\circ} \mathrm{C}$. and above.

Sensation. (a) Sensibility to painful stimuli.--Sensation was profoundly affected over the left lower extremity. Over the area shown in Fig. 47, he was entirely insensitive to the painful aspect of a pin prick; but over the sole of the foot and over the back of the leg he was certainly sensitive to painful cutaneous stimuli. In the direction of the trunk, and also towards the dorsum of the foot, the boundaries of this area of complete cutaneous analgesia sbaded gradually into parts of normal sensibility.

(b) Thermal sensibility.-Over the area in Fig. 48, he was 
insensitive to a test-tube containing ice water, but the borders of this area were not rigidly defned, excepting over the outer side of the thigh. Within these parts, he was also insensitive to all temperatures which caused a sensation of coolness over the normal leg.

Temperatures of from $30^{\circ}$ to $70^{\circ} \mathrm{C}$. produced no sensation of heat over the area in Fig. 49. But he complained that with a stimulus of above $50^{\circ} \mathrm{C}$. pain was produced over all those parts insensitive to heat, but sensitive to cutaneous painful stimuli. Thus, it was possible to map out the area of cutaneous analgesia with a test-tube containing water at $60^{\circ} \mathrm{C}$.

(c) Tactile sensibility was everywhere perfect, both to light and deep touch.

(d) The vibrations of a tuning-fork were every where accurately appreciated.

(e) The sense of passive position and movement was nowhere disturbed.

(f) To the compass-test his answers were equally good over both halves of his body.

(g) Cutaneous localisation was equally good over synonymous parts of the body and limbs.

Spine.-A radiograph showed changes in the body of the sixth cervical vertebra.

\section{Condition in October, 1906.}

Except that his general health improved, his condition remained unchanged. But, as some additional observations of interest were made in the autumn of 1906 , especially those relating to paradox cold, we shall recapitulate shortly his condition at that time.

Motion.-He walked with such ease that he asserted he was as well as ever. The grasps of the two hands were of equal strength.

Reflexes.--The right wrist-jerk was perhaps slightly more active than the left, and the knee-jerk was certainly brisker on the right side than the left. No ankle-clonus could be obtained, and whilst the left plantar reflex was certainly flexor in type, the right was indeterminable.

Sensation. (a) Sensibility to painful stimuli.-The loss of sensation to the prick of a pin and to the painful interrupted current corresponded exactly to that on Fig. 47.

The algometer gave the following readings, showing how closely the decrease in sensibility to the painful aspect of excessive pressure corresponded with the cutaneous analgesia (compare Fig. 47 with the algometer readings). 


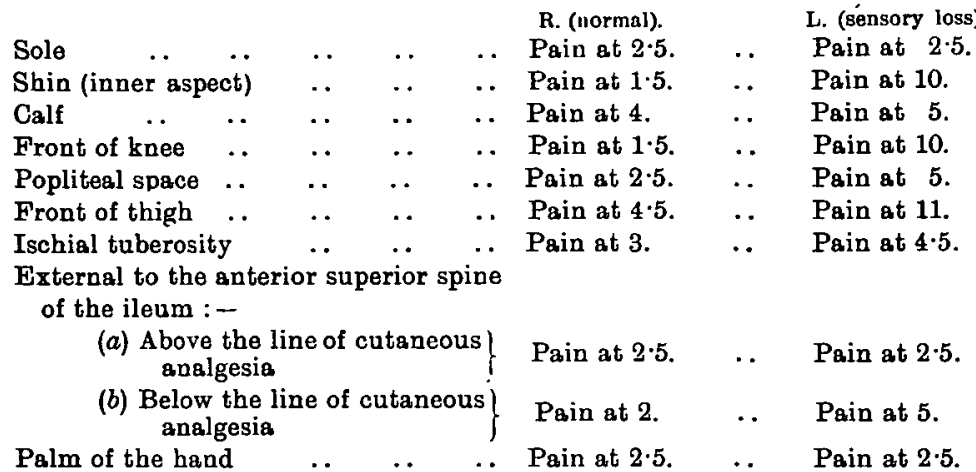

(b) Thermal sensibility. - Some striking experiments were made to elucidate the phenomenon known as "paradox cold." The area insensitive to all cold stimuli was of considerably less extent than that insensitive to heat (Fig. 48 and Fig. 49). Thus, the whole of the abdomen and lower part of the thorax on the left side enclosed by a dotted line on Fig. 48 was sensitive to cold, but not to heat. So long as the stimulus was $40^{\circ} \mathrm{C}$. or below, it produced no sensation over this area insensitive to heat; it was called in every instance "a touch." But, as soon as temperatures of over $45^{\circ} \mathrm{C}$. were employed, the patient invariably said the stimulus was " cold." If the temperature was raised to $55^{\circ} \mathrm{C}$., he complained of " cold pain."

These observations show that, in this instance, the paradox cold obtained by the application of heat to an area insensitive to heat was due to stimulation of cold spots. Such spots reach normally to temperatures above about $40^{\circ}$ with the production of a sensation of coldness.

(c to g).-Every other sensory quality was perfect, as was the case in 1905 .

Case 8.-G. G. A.

In August, 1904, whilst in India, he began to suffer from fever : this continued till November.

January 31,1905 , he was suddenly seized with violent pain in the neck, and lost power in the left arm and left leg, together with sensibility to pain, heat and cold over the right leg and right half of the body.

When first seen in April, 1905, he was a characteristic instance of Brown-Séquard paralysis.

vol. XXIX. 
Righiv.

No motor disturbance.

Knee.jerk normal.

No ankle-clonus.

Plantar reflex flexor in type.

Loss of sensibility to cutaneous painful stimuli (Fig. 50) and to cold (Fig. 51).

No loss to heat.

All other forms of sensation perfect.
Left.

Slight spasticity of left leg. Slight wasting of left hand. Knee-jerk greatly exaggerated. Ankle-clonus. Plantar reflex extensor in type. Sensation perfect.

G. G. A., aged 45, a medical man, was in perfect health until August, 1904. He then began to suffer from pyrexia; his temperature never rose above $100^{\circ} \mathrm{F}$. $\left(37^{\circ} \mathrm{C}\right.$.), but never remained normal for long. He was in India at the time, and the condition was supposed to be malarial, but quinine was entirely without effect. The pyrexia continued until the nuiddle of November, when he thought he was well and resumed his usual duties.

On January 31,1905 , he was suddenly seized with violent pain slightly to the left of the spine of the seventh cervical vertebra : it radiated down the back of the shoulder and up the neck and was worse at night than in the day. This pain increased rapidly for two weeks and his temperature rose again, rising and falling each day as before.

Then the pain became easier, and he was able to sit up and play the banjo. Within two days, his left arm became paralysed, but the pain continued to decrease in severity. The paralysis spread to the left leg, and at the same time the whole right half of the body as high as the nipple became insensitive to pain, heat and cold.

At no time was the motor power of bis right leg diminished, and he had no difficulty in passing or in holding his water.

The right half of the face did not sweat.

About the middle of March, 1905, the paralysis began to pass away from the left arm. By the middle of April, 1905, his temperature ceased to rise above $99^{\circ} \mathrm{F} .\left(37 \cdot 2^{\circ} \mathrm{C}.\right)$, and since that date he has steadily improved.

At the age of 18 he had an attack of venereal disease under a long foreskin; this produced phimosis, for which he was circum- 
cised. Otherwise he was not treated and suffered no consequences.

Condition in April, 1905.

When he was first seen by one of us in April, 1905, he was a well-built active man, in appearance younger than his age.

There were no abnormal signs in the heart, lungs or abdomen; the spleen was not enlarged and his urine was normal.

Motion. - He walked a little stiffly, in consequence of slight spasticity of the left leg, but could stand firmly on either leg and did not fall when his eyes were closed.

The grasp of the left hand was greater than that of the right. All the interossei of the left band were a little wasted, especially

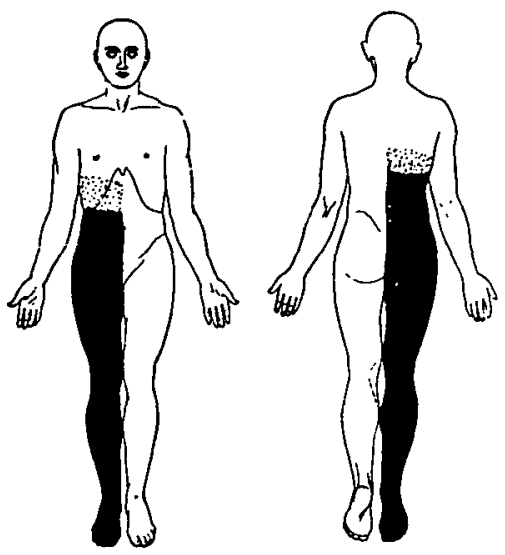

Fia. 50.

To show the loss of sensibility to cutaneous painful stimuli in Case 8. The dotted area represents a band of diminished sensibility.

the first, and the fingers were a little over-extended at the metacarpo-phalangeal, and flexed at the interphalangeal joints. The thenar muscles showed slight signs only of wasting. All the muscles reacted perfectly to the interrupted and constant currents.

Reflexes.-The left knee-jerk was greatly increased compared with the right, and ankle-clonus was definitely present on the left side; no clonus could be obtained from the right ankle. On the left side, the plantar reflex was of the extensor type, whilst that from the right foot gave a flexor response.

Sensation. - He complained of no abnormal sensation, unless the right leg was touched with a cold object; for instance, when 
standing on a cold floor, he noticed a luke-warm sensation in the right foot instead of the normal coldness.

(a) Sensibility to pain.- He was completely insensitive to all cutaneous painful stimuli over the area in Fig. 50. The frontal border was ill-defined, merging into parts of normal sensibility, and the boundary of the analgesia differed according to whether testing was carried out from normal to abnormal parts or vice versâ.

The right testicle and right balf of the penis and scrotum were insensitive to painful stimuli.

(b) Thermal sensibility.-He showed a remarkable dissociation between sensibility to heat and to cold. All temperatures between $35^{\circ} \mathrm{C}$. and $55^{\circ} \mathrm{C}$. could be appreciated everywhere over

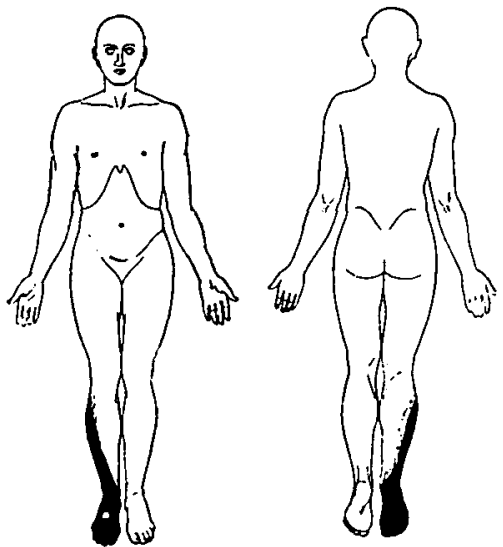

Fig. 51.

To show the loss of sensibility to cold in Case 8 .

the right leg, and $35^{\circ} \mathrm{C}, 45^{\circ} \mathrm{C}$, and $50^{\circ} \mathrm{C}$. could be discriminated.

But, over the area marked on Fig. 51 he was entirely insensitive to all temperatures which normally produced a sensation of coldness.

(c) Tactile sensibility in all its forms was perfect, whether the stimulus was cotton wool or a deep touch.

(d) Acucsthesia.-He could tell the head from the point of a pin everywhere over the body and limbs.

(e) The sense of passive position and movement was everywhere perfectly preserved.

(f) Tactile localisation was equally good over both lower extremities. 
Spine.-He had great difficulty in turning his head to the left until a few weeks before he was first seen. Even then he could not turn it rapidly. The radiograph gave results that were uncertain, all that could be said was, that the shadow was somewhat more blurred on the left side than on the right in the region of the transverse processes of the tifth and sixth cervical vertebræ.

Sphincters.- He has had no trouble with micturition and defæcation.

\section{Condition in October, 1906.}

He greatly improved, and was last seen in October, 1906.

Motion.-The left leg no longer gave him trouble, and was not stiff or spastic ; he could run upstairs " two at a time."

Reflexes.-The reflexes were unchanged, still greatly increased in the left lower extremity.

Sensation. (a) Sensibility to pain.-The area insensitive to prick bad greatly decreased, and,.although over the back and front of the right thigh and over the sole of the foot the sensation was diminished, the only absolute loss of cutaneous painful sensibility lay over the leg between the knee and the ankle.

This was associated with a diminution in sensibility to the pain of pressure compared with the left leg, as sbown by the following algometer readings :-

\begin{tabular}{|c|c|c|c|c|c|c|c|c|c|}
\hline \multirow[b]{2}{*}{ Sole } & \multirow[b]{2}{*}{.. } & \multirow[b]{2}{*}{.. } & \multirow[b]{2}{*}{.. } & \multicolumn{4}{|c|}{ R. (sensory loss). } & \multicolumn{2}{|r|}{ L. (motor) } \\
\hline & & & & $\cdots$ & Pain at & $7 \cdot 6 \cdot 5$ & $\cdots$ & $\cdots$ & Pain at $2-2 \cdot 5$. \\
\hline Shin & ner asp & pect) & . & .. & Pain at & 4 & .. & .. & Pain at $1 \cdot 5-2$. \\
\hline Calf & .. & .. & .. & .. & Pain at & $5-6$ & .. & .. & Pain at $3-3 \cdot 5$. \\
\hline Kne & $\ldots$ & .. & .. & .. & Pain at & $10-7$ & .. & .. & Pain at 4-3. \\
\hline Popl & 1 space & $\ldots$ & .. & . & Pain at & 3.4 & .. & .. & Pain at $2 \cdot 5-2 \cdot 5$ \\
\hline Ilize & & .. & $\ldots$ & .. & Pain àt & $7-6$ & .. & .. & Pain at 3-3. \\
\hline Butt & & .. & .. & .. & Pain at & $3-3$ & .. & .. & Pain at 2:5-2. \\
\hline Low & & . & .. & .. & Pain at & 1.5 & . & .. & Pain at 1.5 . \\
\hline Palm & .. & .. & .. & .. & Pain at & $3-3$ & .. & . & Pain at $3-3$ \\
\hline
\end{tabular}

These readings show no absolute loss of sensibility to the pain of pressure, but all those from the right leg are uniformly somewhat higher than those from similar parts of the left leg. Two readings are given to show the manner in which the algometer results vary when sensation is not abolished.

(b) Thermal sensibility. - Anæsthesia to cold was still present over the external aspect of the right leg and dorsum of the foot, but no longer occupied the sole. Over this area of loss of sensation, water at $20^{\circ} \mathrm{C}$. and below was uniformly called "warm."

$(c-f)$.-Every other sensory function was perfect. 


\section{Case 9.-Joseph F.}

A glio-sarcoma of the central gray matter of the spinal cord extended from the medulla to the seventh thoracic segment. Situated for the greater part in the centre of the cord, it had invaded and destroyed the left half in the region from the third to the seventh cervical segments.

It was found on his admission to the hospital that motion was lost in the left arm, but it was not until shortly before his death that the right arm became paralysed.

Both knee-jerks were increased; ankle-clonus was obtained on both sides, and both plantar reflexes gave an extensor response.

For some time after admission, the local disturbance of sensation consisted of analgesia and thermo-ancesthesia of the left upper extremity, with abolition of the sense of passive position and move. ment and of tactile discrimination. Tactile sensibility was preserved. Shortly before death, tactile sensibility was also lost in this arm.

The remote disturbance of sensation consisted throughout of loss of sensibility to pain, heat and cold over the right leg, right half of the body and right arm and hand.

The sense of passive position was greatly diminished in the left foot, but perfect on the right side.

Joseph F., aged 44, an actor and vocalist, was admitted to the London Hospital under the care of Dr. Dawson on July 6, 1905, and died on August 10.

In November, 1904, he first noticed slight weakness and stiffness in the left arm with a tingling sensation in the fingers of the left hand. Shortly afterwards his neck began to grow stiff.

Until February, 1905, he was able to play the piano with both hands, but the left arm grew steadily weaker; slight weakness appeared in the right arm just before admission.

On two occasions within the fortnight before admission he passed urine involuntarily.

He had suffered from no previous illness, except an attack of venereal disease, accompanied by warts, but followed by no secondary consequences. He had never taken alcohol to excess.

Condition shortly after Admission.

He was an intelligent man, interested, until shortly before his death, in his condition and the testing of bis sensation. His 
speech was unaffected, and he never suffered from fits or headache.

Motion.-The left arm lay flaccid and motionless on the bed beside him. The small muscles of the left band were wasted, and the left arm was nearly $1.5 \mathrm{~cm}$. smaller in diameter than the right. No fibrillary twitchings were visible, and the muscles of the left arm and hand reacted perfectly both to the interrupted and to the constant current.

The small muscles of the right band were wasted and the grasp was weak; but all movements of the thumb and fngers and of the rest of the upper extremity could be made without difficulty.

Both legs were distinctly spastic but were not paralysed; the muscles were flabby but not wasted.

Reflexes.-Both knee-jerks were increased, ankle-clonus was obtained on both sides, and both plantar reflexes gave an extensor response.

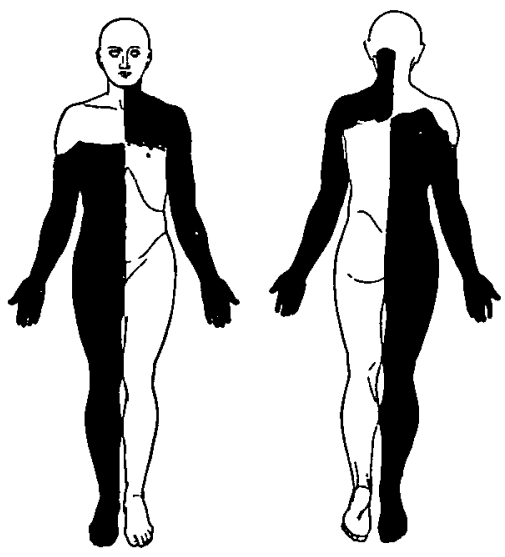

Fig 52.

To show the loss of sensation to cutaneous painful stimuli in Case 9.

Sensation.-(a) Sensibility to pain.-He was insensitive to all cutaneous painful stimuli, such as the prick of a pin or the interrupted current, over the whole of the right leg, right half of the body and right arm (Fig. 52). On the opposite side, the analgesia occupied the left arm and left half of the neck.

Excessive pressure produced no pain over either palm, but the amount of the pressure applied was not measured with the algometer.

(b) Thermal sensibility.-Sensibility to beat and cold was 
abolished over the area shown in black on Fig. 53. Over the right side of the neck it was greatly diminished, more so to heat than to cold.

(c) Tactile sensibility was unimpaired at this time; even cotton wool was appreciated over the left arm and hand.

(d) Sense of passive position and movement.-He was unable to recognise the position of any part of the left upper extremity, and was ignorant of even the grossest movements; but in the right hand and arm the sense of passive movement and position was perfect.

His power of recognising movements of the left great toe was defective, but from the ankle and knee his answers were correct.
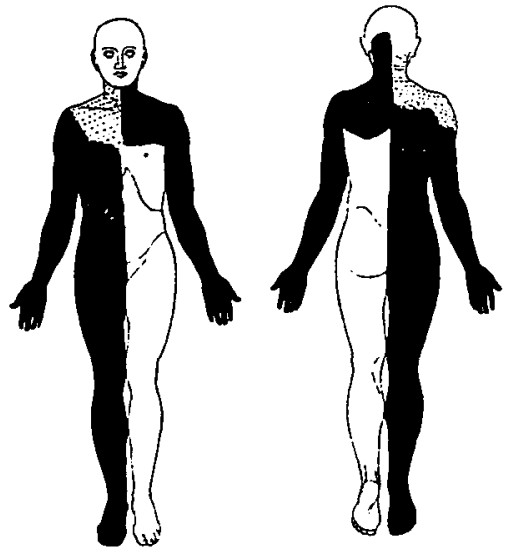

Fig. 53.

To show the loss of sensation to heat and cold in Case 9.

On the right side he was uniformly accurate in his appreciation of movements of the great toe, ankle and knee.

(e) Compass-test.-Tactile discrimination was perfect in the right hand, and he made two mistakes only when the compasspoints were separated to a distance of $1 \mathrm{~cm}$. But over the left hand the two points were called one even when $5 \mathrm{~cm}$. apart.

L.

$\mathbf{R}$

Palm (applied transversely) $\quad 5 \mathrm{~cm} \cdot \frac{1 \mid 10 \mathrm{R} .}{2 \mid 10 \mathrm{~W} .} \quad 2 \mathrm{~cm} \cdot \frac{1 \mid 10 \mathrm{R}}{2 \mid 10 \mathrm{R}}$

$1 \mathrm{~cm} . \frac{1 \mid 9 \mathrm{R} .1 \mathrm{~W} .}{2 \mid 9 \mathrm{R} \cdot 1 \mathrm{~W} .}$

Cranial nerves.-The pupils reacted to light and accommodation, and the ocular movements were good. There was no paralysis of the face, and the tongue was protruded well. He could move both halves of the palate and at this time swallowed well. 
Subsequent progress. - He gradually lost power in the right arm and hand, and although every movement could be carried out feebly, could at last no longer feed himself. Tactile sensibility became so greatly diminished in the left arm that the compass. lest could not be applied; he gave no answer when touched with the two points. Over the whole of the left upper extremity, he now gave no response to the pressure with a pin, whereas previously every prick was said to be a touch.

The extent of the insensibility to pain, heat and cold remained as before.

Gradually complete incontinence of urine and fæces came on and swallowing became difficult.

By August 9 the right arm was completely paralysed and on the 10 th he died suddenly.

\section{Post-mortem Examination.}

An autopsy was made on August 11. Rigor mortis was present. No abnormalities were found in the lungs, heart, liver, kidneys, or spleen.

The brain and spinal cord were removed together by sawing through the occipital bone. The spine was found to be entirely unaffected. But it was at once obvious that the spinal cord was greatly enlarged in the cervical region, and this increase in size extended up to the bulb. During the manipulations a considerable quantity of clear fluid escaped.

No changes were found in the cerebrum or cerebellum.

On microscopical examination a growth was found extending from the region of the olive to the eighth thoracic segment, situated in the gray matter around the central canal. But, even at levels as low as the third lumbar, the tissue around the central canal was abnormally extensive. Throughout the cord, the growth lay in the gray matter forming at the first and second cervical level a well-defined round tumour in the centre of the spinal cord. But, at the third cervical level, it had invaded and destroyed the whole of the left half of the spinal cord; the right half was much compressed. This destruction, more particularly of the left half of the cord, extended to the level of the seventh cervical. Below this, the tumour again assumed a more central position and could be clearly seen to the seventh thoracic level. Below this, some increase in the tissue around the central canal alone marked the position occupied by the tumour in higher segments. When stained by the Weigert-Pal method the whole of the crescentric portion representing the displaced right half of 
the spinal cord on Figs. 1 and 2 [Plate 1] took the stain well, and showed large numbers of apparently normal fibres. In the dorsal region this stain showed profound degeneration of the pyramidal trace in the left half of the cord; in the right half this tract took the blue stain well. [Plate 1.]

The growth consisted of cells with a large deeply-staining nucleus and a small amount of protoplasm. Heidenhain's ironhæmatoxylin method showed that many of these cells possessed processes which interlaced. It was probably a glio-sarcoma starting in the tissue around the central canal. Hæmorrhage had taken place at several points within the growth.

\section{CASE 10.-J J $\mathrm{J}$ ES $\mathrm{Y}$.}

July, 1906, he began to lose power in his legs, first in the left and then in his right; at the same time he ceased to be able to pass his water, and developed retention of urine with overflow.

Admitted to Hospital with a high temperature and signs of bronchitis in both lungs.

He improved and between August 10 and 15 complete examination was possible.

Absolute paralysis of motion was present below the umbilicus.

There was much loss of sensation to painful and thermal stimuli over both lower extremities (Fig. 54).

Sensibility to light touch was perfect except over left foot.

He had lost all appreciation of passive position and movement in the lower limbs, and was unable to discriminate two points separated to more than twice the normal distance.

Yet he could name the spot touched accurately, although ignorant of the position of his legs.

On post.mortem examination, a metastatic growth secondary to a hypernephroma of the right kidney was found in the left half of the spinal cord. This had caused hemorrhage, which extended widely in the left half of the cord from the seventh thoracic to the second lumbar segment.

Profound acute degeneration was found in both posterior columns, in both lateral bundles of Gowers and in both direct cerebellar tracts.

James Y., aged 61, was admitted to the London Hospital on July 27, 1906 (Dr. Schorstein).

For about a month he had complained of pains in the back, chiefly in the lower dorsal and upper lumbar region. Three 


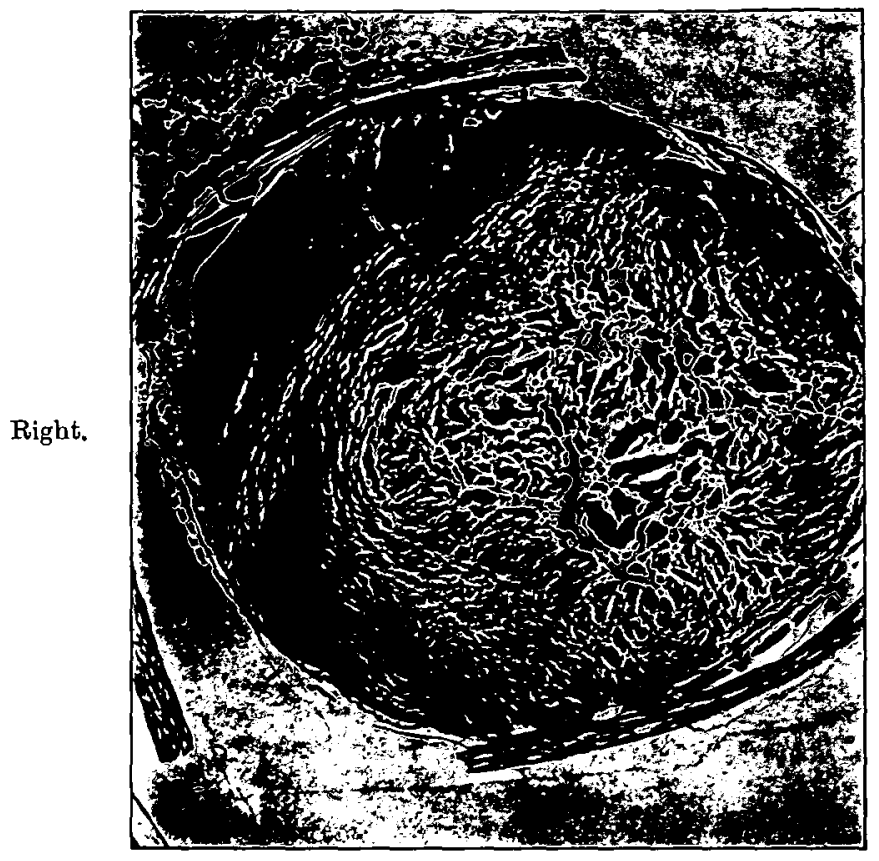

Left.

Fig. 1.-Cervical 3.

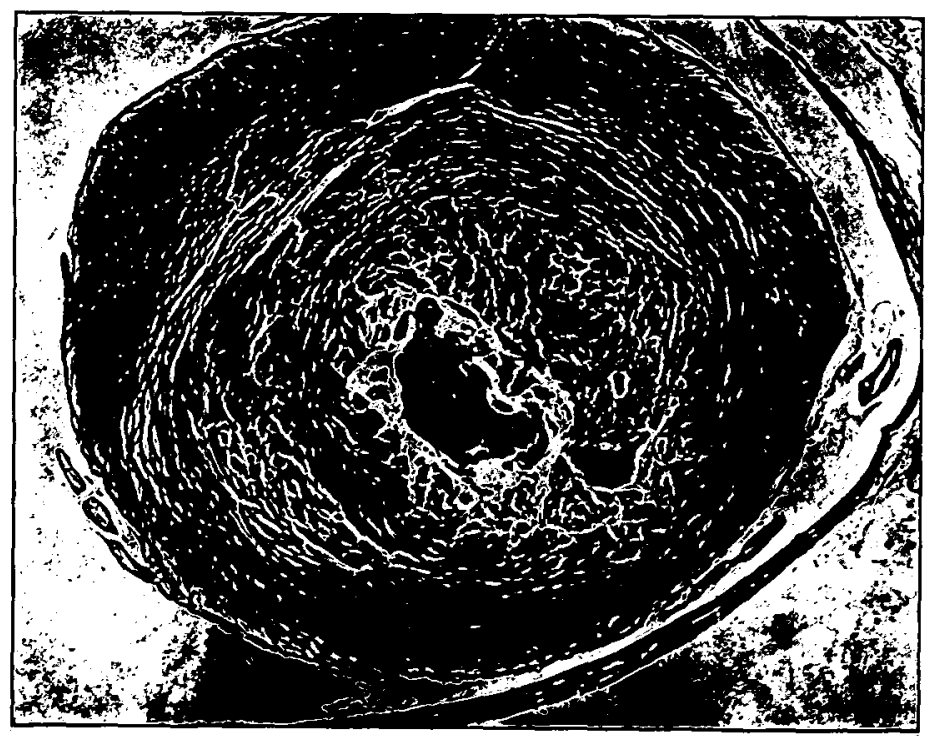

Fig. 2.-Cervical 6. 


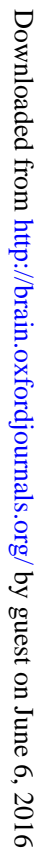


weeks before admission he began to lose power, first in the left and then in the right leg. At the same time he noticed that he was unable to pass water, and yet as he stood it would run from him. Occasionally, during the three weeks before admission, he had lost control over his motions.

He was a file-cutter, but bad never suffered from any form of lead poisoning. No history of venereal disease could be obtained ; he had been married for 34 years and had five healthy children. He denied all excess of alcohol, and had the appearance of a man of temperate habits.

\section{Condition on Admission.}

When first admitted in July, 1906, his temperature ranged between $100 \cdot 2^{\circ} \mathrm{F}$. and $102.4^{\circ} \mathrm{F}$. $\left(37.8^{\circ} \mathrm{C}\right.$. and $39^{\circ} \mathrm{C}$.). He lay propped up in bed with flushed cheeks, breathing rapidly; the pulse was 120 in the minute and regular. Both lungs were emphysematous and râles were widely heard, but at first no signs of consolidation were discovered. The heart-sounds were in no way abnormal. The bladder was distended almost to the umbilicus, and the urine drawn off by catheter was faintly acid and contained some mucus and urates. Over the sacrum was a large bed-sore.

Progress.-During the first fortnight his urine was drawn off regularly, and he rapidly improved under treatment. The temperature gradually fell, so that from August 10 to August 15 it did not rise above $99^{\circ} \mathrm{F}$. $\left(37 \cdot 2^{\circ} \mathrm{C}\right.$.). During this period he was so much better that careful observations could be made upon the condition of sensation; he became cheerful, interested in the testing of sensation, and remarkably trustworthy in his answers, if precautions were taken that he should not become exhausted.

But on August 15, the temperature began to rise and on the 16 th he had a rigor. Further rigors followed on the 18th; definite signs of pneumonia appeared in the right lung, and he died on August 20.

\section{Observations on the Condition of the Nervous System made between August 10 and August 15.}

The patient was intelligent and answered well to the controltests applied. At the time the following observations were made, his temperature was normal. He was bright and cheerful, with the hope that he was on the way to recovery.

Sensation.-He complained of no pain or abnormal sensations in the lower extremities, which were alone the seat of gross sensory defects. 
(a) Pain.-The prick of a pin was not appreciated over the area shown in Fig. 54. Below the kuees, the loss of sensation was absolute, merging gradually above into parts of normal sensibility. Over the front of both thighs he could appreciate the pain produced by a prick, but sensation appeared to be diminished; above the level of the groin it was entirely unaffected.

This distribution exactly agreed with the analgesia to the painful interrupted current. Over both lower extremities below the level of the groin, sensation was somewhat diminished, but the only complete loss of sensation to this stimulus corresponded to the parts marked on Fig. 54 .
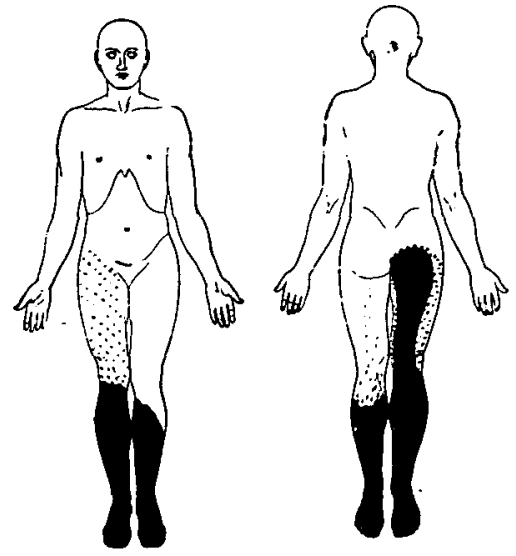

Fig. 54.

To show the loss of sensation to cutaneous painful stimuli in Case 10.

The algometer showed that pain was produced when pressure reached the following amount, measured in kilograms.

R.

L.

Sole of foot $\quad \ldots \quad \ldots \quad \ldots \quad \ldots 10$ (dull pain) $\quad \ldots 10$ (dull pain).

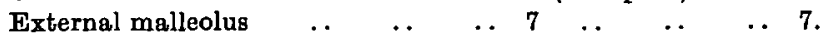

Inner aspect of tibia $\quad \ldots \quad$.

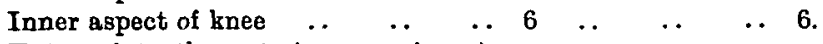

\begin{tabular}{c}
$\begin{array}{c}\text { External to the anterior superior } \\
\text { spine of the ileum }\end{array}$ \\
\hline
\end{tabular}

Head of metacarpal bone of thumb $\quad . \quad 5$ (sharp pain) $\quad . \quad 5$ (sharp pain).

(b) Heat and cold.-The parts insensitive to a temperature of $50^{\circ}-\tilde{0} 5^{\circ} \mathrm{C}$. corresponded to the blackened portions of Fig. 54 ; but the whole of the right thigh was also insensitive to water at $40^{\circ} \mathrm{C}$. 
The loss of sensation to temperatures between $0^{\circ}$ and $25^{\circ} \mathrm{C}$. corresponded on the left lower extremity with the anæsthesia to heat. On the right leg, the loss of sensation extended up to the groin; over the right thigh, stimulation with ice-cold water was invariably said to be "hot." Thus a series of tests with silver tubes, containing water at different temperatures, gave the following results: ice was called " hot," a temperature neutral to the normal skin was called "a touch," $55^{\circ} \mathrm{C}$. appeared to be hot, $40^{\circ} \mathrm{C}$. was said to be "a touch." At the time these tests were made, the temperature of the room was $24^{\circ} \mathrm{C}$.

Here we find an instance of the rule that, when one of the two qualities of thermal sensibility is abolished, extreme degrees will be called by the name of the range of temperature which can be appreciated. Sensibility to cold $\left(0^{\circ}\right.$ to $25^{\circ} \mathrm{C}$.) was abolished over the right thigh, although $50^{\circ} \mathrm{C}$. could be appreciated; the application of ice-cold water to this thigh invariably evoked the statement that the stimulus was hot.

(c) Light touch.-When first examined there seemed to be no part of either lower limb where he could not appreciate a light touch with cotton wool. But, on August 13th, he was somewhat insensitive to this stimulus over the left foot below the level of the ankle; elsewhere, however, even on this date he showed no trace of diminished sensibility to cotton-wool.

(d) Deep touch and pressure were everywhere appreciated, and be never failed to answer correctly throughout the whole period he was under examination.

(e) Passive position and movement.-He was entirely unable to recognise the position into which his lower limbs had been placed passively, and was unconscious that they had been moved. Extensive movements could be made at the ankles, knees and hips without his knowledge. If his eyes were closed, the legs could be moved from the extended position in any direction and the knee flexed through $40^{\circ}$, and he still imagined they lay stretched out before him on the bed. When he was allowed to open his eyes, his expression of surprise amply testified to the greatness of his error.

He was equally unable to recognise movements of the feet or legs produced by electrical stimulation of the muscles of the lower limb.

The appreciation of passive position and movement was not disturbed in any part of the body, except from the hips downwards.

( $f$ ) Compass-test.-Over the palm of the left hand, which we used as a control, he gave perfect answers when the compass 
points were separated by $1.5 \mathrm{~cm}$. and applied longitudinally. But, over the outer aspect of the left leg, he failed entirely at $15 \mathrm{~cm}$., and over the front of the left thigh when they were $20 \mathrm{~cm}$. apart.

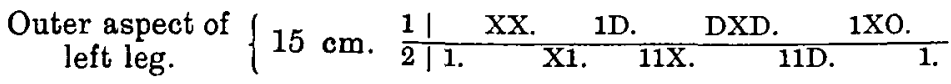
or $\quad \frac{1 \mid 2 \text { Right. } 3 \text { Doubtful. } 4 \text { Wrong, and once no answer. }}{2 / 7 \text { Right. } 1 \text { Doubtful. } 2 \text { Wrong. }}$

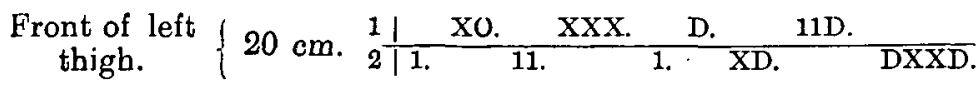

or $\quad \frac{1 \mid 2 \text { Right. }}{2 \mid 4 \text { Right. }} 3$ Doubtful. 4 Wrong, and once no answer.

(g) Cutaneous localisation.-On several occasions we satisfied ourselves that he was able to name the spot touched with accuracy; but one sitting was devoted to the following experiments.

(1) He was allowed to see the position of his limbs before they were touched. Then his eyes were closed and he was touched at similar spots on the two lower limbs, on the sole, the instep, the dorsum of the foot and just below the knee. In every case he named correctly the part touched.

(2) His eyes remained closed and his legs were moved so that he was unable to say in what position tbey lay. Then he was touched on the sole, the dorsum of the foot, the calf and just below the knee, first on the one limb and then on the other. In every instance his answers were correct. In fact, no difference could be discovered in the ease with which he named the spot touched when he knew how his legs were lying, and when he was totally ignorant of the position into which they had been placed.

He was never mistaken in naming the side to which the limb belonged that had been touched.

(3) His eyes were closed and the limbs were moved so that the left leg was flexed at the knee and hip, the right leg extended and abducted. Various points on the legs were touched and he was asked to indicate the spot with a light cardboard rod. The rod struck the bed idly; for so great was the error he was unable even to touch the legs, and yet he continued throughout to name correctly the spot touched.

(h) Acucasthesia.-He was able to distinguish the head from the point of a pin all over both lower limbs, even over parts which were entirely insensitive to all painful stimuli. 
(i) Tuning-fork.-The vibrations of a tuning-fork beating 128 were everywhere appreciated.

Motion.-Below the level of the umbilicus he was completely paralysed, and could make no movements of the hips, knees, ankles or toes. The legs lay flaccid, neither wasted nor spastic, all the muscles reacting normally to the interrupted and constant current.

Every other movement was perfectly performed and there was no wasting of the hands or arms.

Reflexes.-The knee-jerks were abolished, but ankle-clonus was obtained on both sides. Wrist and elbow-jerks were in every way normal.

The plantar reflexes gave an uncertain response.

Cranial nerves.-The pupils were equal and reacted to light and accommodation. Ocular movements were perfect. There was no affection of the face and tongue.

No changes were found on ophthalmoscopic examination.

Sphincters.-On admission to the hospital, his bladder was greatly distended and be never regained the power of passing his water. It was drawn off twice daily by catheter.

The bowels were obstinately confined, but when opened by enemata he was unable to retain his motions.

\section{Post-mortem Examination (August 21).}

The body had been preserved in the cool chamber at a temperature just above freezing point. Rigor mortis was present. Over the sacrum and buttocks was a large bed-sore which was present on the patient's admission to the hospital.

The right lung was almost solid from septic broncho-pneumonia, and within it, at the extreme base, was found a small abscess-cavity of about the size of a walnut. This lung was adberent to the chestwall except at the base ; here there was a small collection of pus.

The left lung was widely adherent and contained patches of septic broncho-pneumonia.

The larynx and trachea were healthy.

No abnormality was found in the pericardium or heart, but the aorta was somewhat atheromatous.

No fluid was found in the abdominal cavity. The spleen was much enlarged and weighed $14 \frac{1}{2}$ ozs. (411 grms.); its substance was softened. No abnormal changes were found in the liver, which was not enlarged.

On removing the right kidney a large encapsuled growth was seen adherent to and distorting its upper surface. This growth was about the size of a walnut, soft and of a ligbt yellow colour. 
On its inner aspect was seen the suprarenal capsule. In the lower part of the same kidney was a secondary growth, enclosed in a capsule, and of the same yellow colour.

On microscopical examination, the tumour was found to be a hypernephroma formed of columnar cells arranged in columns. Extensive bæmorrhage separated these columns of cells. Many of the cells contained brown granular material, apparently altered blood pigment.

The left kidney sbowed no gross abnormality.

No changes in the brain could be seen with the naked eye; it contained no secondary growths.

Condition of the spinal cord.-The cord was removed and bardened in 5 per cent. formalin. No growth or other abnormal changes were present in the membranes and no gross disease could be discovered before the cord was incised. But, as soon as it was cut across at the level of entry of each pair of roots, profound changes were at once visible, consisting firstly of hæmorrhage around a growth, and secondly of obvious degeneration in the posterior columns, which were whiter than the rest of the cord.

Distribution of the focal lesion (Plate 2).-The first sign of a focal lesion was found at the level of the seventh thoracic; here the base of the left posterior horn was occupied by an extravasation of blood. On both sides, the vessels of the posterior and lateral columns in the neighbourbood of this hæmorrhage were dilated and unduly visible.

At the level of the eighth thoracic, this hæmorrhage had spread so that it occupied the apex of the triangle formed by the left posterior column, but did not encroach on the lateral column.

At the ninth thoracic level, the bæmorrhage was rather more extensive, and for the first time dark granules could be seen amongst the extravasated blood.

At the level of the tenth thoracic, the lesion had reached such proportions that the whole of the left posterior horn and the greater part of the lateral column was destroyed. The external portions of the lateral column stained well by the Weigert-Pal method, but the whole of the blunt angle between the anterior and posterior horns was filled up with extravasated blood, which had also completely destroyed the central portion of the posterior horn. The posterior column was pressed upon and displaced, but took the Weigert stain well. Within the hæmorrhagic area were two foci, which consisted of small cells containing pigment. In sections stained by Van Gieson's method, the disease was seen to 


\section{PLATE II.}

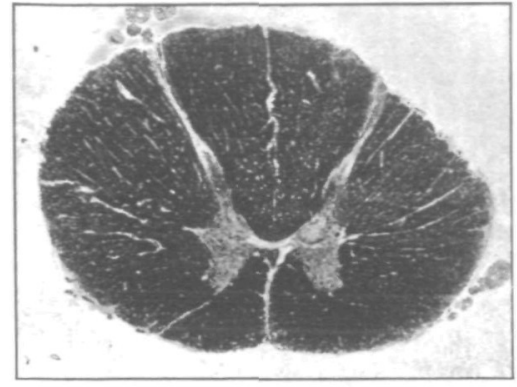

FIG. 1.- Thoracic 6.

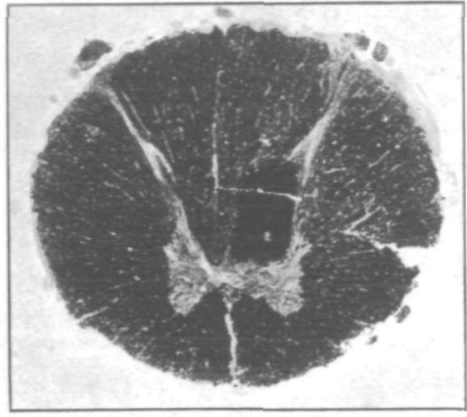

FIG. 2.-Thoracic 8.

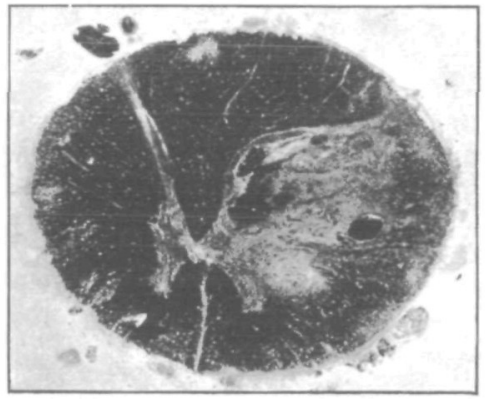

FIG. 3.-Thoracic 10.

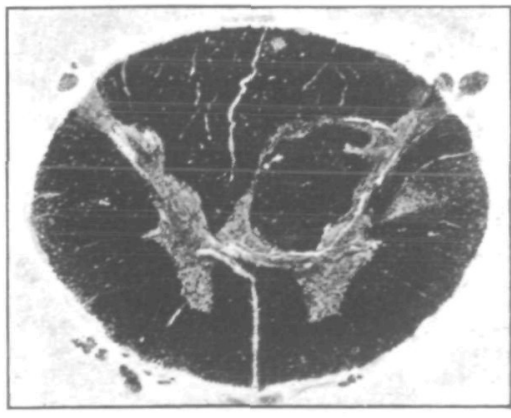

Fig. 4.-Dorsal 12.

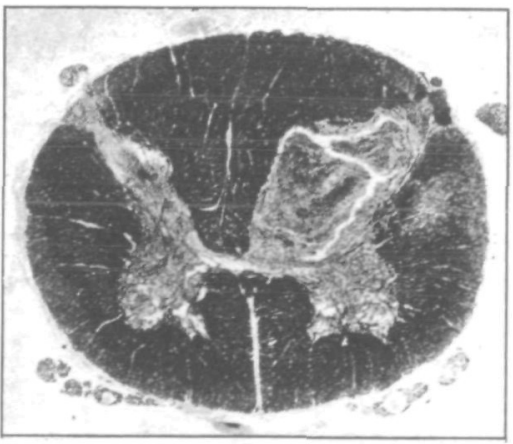

Fig. 5.-Lumbar 2. 
PLATE III.

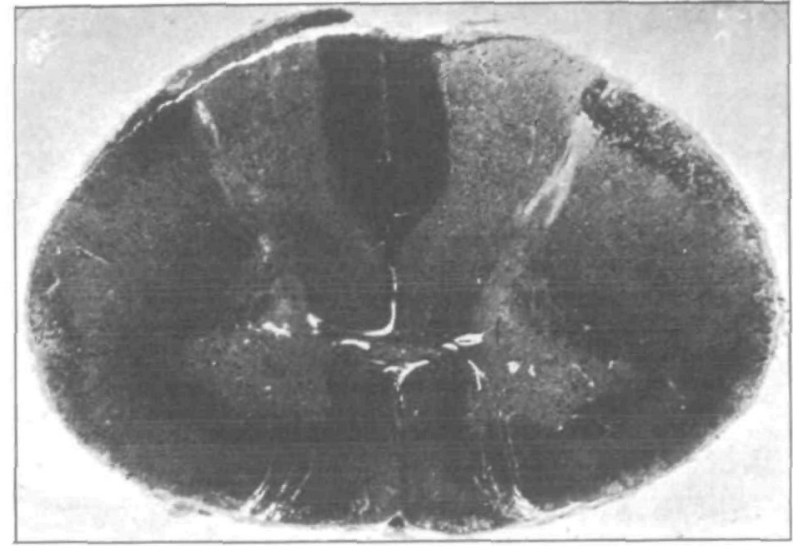

FIg, 1,-Cervical 4.

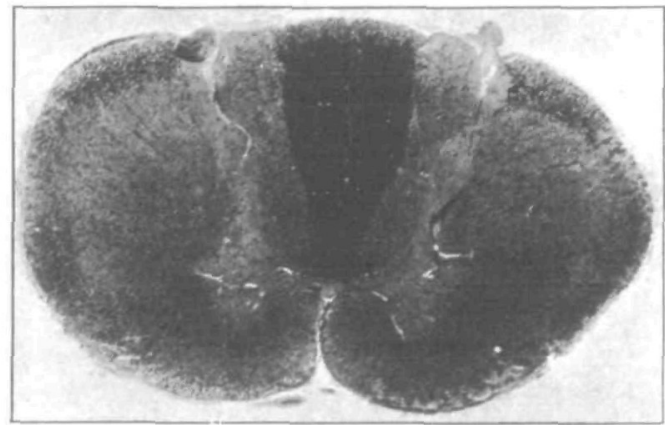

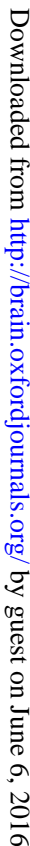

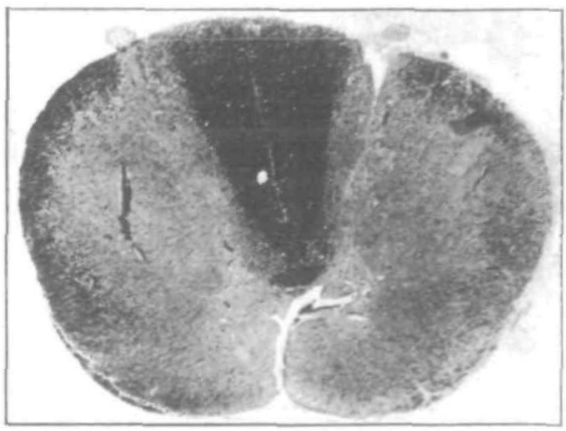

FIG, 3.-Thoracic 4.

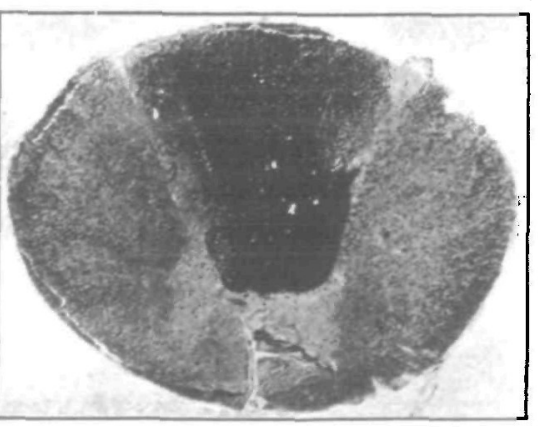

FIG. 4.-Thoracic 7. 
have invaded the whole of the gray matter on the posterior and left lateral aspects of the central canal.

At the level of the twelfth thoracic, the hæmorrhage had assumed the form of an oval ring, which entirely separated the base of the posterior horn and a portion of the left posterior column from the remainder of the spinal cord. Within this ring of extravasated blood lay fibres which stained blue with the Weigert-Pal stain.

At the level of the first lumbar, the hæmorrhage occupied about the same extent, but within its limits the tissue was more profoundly disintegrated.

At the level of the second lumbar, the hæmorrbage had destroyed the whole of the left posterior horn and a portion of the left posterior column. No definite signs of growth could be seen at this level. .

From this point downwards, the cord showed no signs of a focal lesion.

Distribution of the systemic degeneration. [Plate 3.] -At the level of the seventh thoracic, staining by Busch's modification of Marchi's osmic acid method showed degeneration in the whole extent of the posterior columns on both sides. At this level, the extreme upper end of the focal lesion could be seen as a hæmorrhage (Fig. 1) in the left posterior horn. But in spite of the apparently unilateral position of the focal lesion, both posterior columns were profoundly and equally degenerated.

At the level of the fourth thoracic, the root-zones and greater part of the postero-external columns were seen to be free from degeneration, which occupied mainly the postero-internal columns on both sides; but the cornu-commissural tracts were degenerated.

At the level of the first thoracic, the degeneration did not extend into the postero-external columns, but, with this exception, occupied the whole of the posterior columns. The cornu-commissural tracts were slightly degenerated only. [Fig. 3.]

At the level of the fourth cervical, the degeneration occupied the postero-internal columns only, and mainly their dorsal portions; the degeneration in the more ventral portions was much less than in the lower segments. The cornu-commissural tract was not degenerated. [Fig. 4.]

Above the level of the seventh thoracic, both lateral bundles of Gowers and the direct cerebellar tract of both sides showed profound degeneration, slightly greater in the left than in the right half of the cord. This degeneration extended throughout the cervical region and is visible in Figs. 2, 3, and 4. [Plate 3.]

There was definite degeneration in the region of the spino-thal-

voL. XXIX. 
amicus of both sides, greater on the side (right) opposed to the main focal lesion.

Some degenerated fibres were also seen on the ventral surface of the cord, especially in that part traversed by the anterior roots.

Summary of Post-mortem Appearances.

The right lung was solid with septic broncho-pneumonia and a small abscess was found at its extreme base.

The right kidney was the seat of a malignant growth (hypernephroma), characterised by columnar cells and the presence of pigment due to extravasated blood.

Secondary growths were found in the kidney and in the spinal cord.

That in the spinal cord had caused hæmorrhage which bad extended beyond the limits of the metastatic growth. This focal lesion extended from the seventh thoracic to the second lumbar segment, occupying mainly the left posterior horn and the adjacent parts of the posterior and lateral columns. At the level of the tenth thoracic, it reached its maximum extent, destroying the whole of the left posterior horn and a greater portion of the left lateral column.

Acute ascending degeneration was found in both posterior columns.

Both lateral bundles of Gowers, the direct cerebellar tract and apparently the spino-thalamicus, also showed signs of acute degeneration.

\section{Case 11.-Charles H.}

A case of unilateral syringomyelia, which has been under observation from December, 1903.

Motion remains unaffected and he shows no wasting of any group of muscles.

The reflexes are still normal. He suffers from arthropathy of the right shoulder, which at one time gave rise to intense deep tenderness over a part of the analgesic limb.

Sensation to pain, heat and cold was at first lost over the whole of the right upper extremity and the upper part of the chest on the right side. This loss of sensation gradually spread on to the face and scalp. It has always been more extensive to heat and cold than to pain.

Tactile sensibility, the sense of passive position, tactile discrimination and tactile localisation have never been in any way affected. 
Charles H., aged 30, first came under the care of Dr. Head at the London Hospital on December 7, 1903.

Eight years previously he was driving an omnibus; wishing to descend from the box, he missed the strap and fell, striking his right shoulder violently. He experienced "a feeling of numbness" and came to the London Hospital, but no serious injury was discovered. At the end of a week he returned to work.

Some considerable time afterwards, he noticed that his shoulder-joint "clicked" when he moved his arm, although it was entirely painless.

But, about six months before his admission in 1903, he began to suffer pain whenever his shoulder "clicked." The pain was situated not only in the shoulder, but also around the right ear.

Shortly before admission, the right arm and shoulder began to swell and "became set"; when he placed his hand into his pocket, he could not remove it again.

He had had no other illness, and denied all venereal disease. He had been married nine years and had two healthy children.

He has been under observation from December, 1903, until the present time, and we have repeatedly examined him together, and separately. We shall, therefore, give a summary of his condition, noting under each separate beading any change that may have occurred in the condition of the part, or function, of which we are treating.

\section{Physical Condition.}

$\mathrm{He}$ is a splendidly-built man with well-developed muscles. His heart and lungs are sound and he has shown no signs of visceral disease.

Throughout, he has been intelligent and unusually trustworthy to control-tests. His answers over normal parts have always been quick and accurate.

Motion.-Throughout the last three years, motor power has never been affected, except from the pain produced by moving the right arm. The grasps have remained equal and powerful, and no muscles have shown signs of wasting.

He walks well and can stand perfectly with his eyes closed. The legs are neither spastic, paretic, nor wasted.

Reflexes. - The knee-jerks and wrist-jerks have remained normal, and both plantar reflexes have given a flexor response throughout. No ankle-clonus was ever obtained.

Condition of the right shoulder-joint. - When be was admitted to the hospital in 1903, the right shoulder-joint was much swollen 
and evidently contained fluid. A radiograph showed that the head of the humerus had been eroded, so that its surface was concave instead of convex; there was also some excessive formation of bone around the trochanters and articular surface of the scapula. The fluid threw a dark hazy shadow round the joint. On February 8, 1904, a small quantity of fluid was removed from the joint; it was of a pinkish-yellow colour and sterile on cultivation.

A considerable part of the swelling seemed to be due to muscular contraction, which varied from time to time.

At first, the joint was excessively painful, especially when

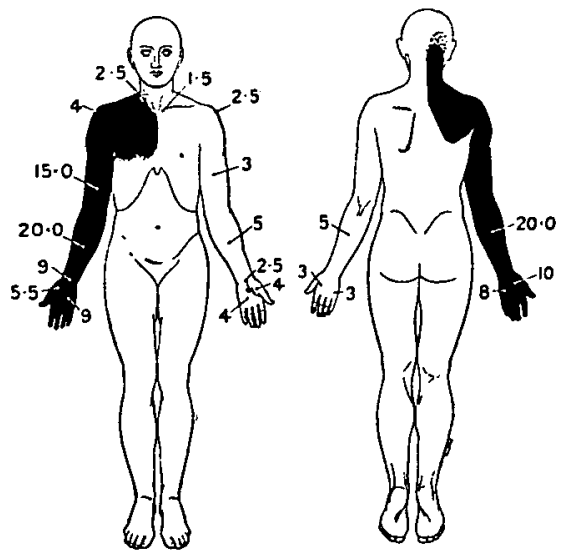

Fig. 55.

To show the loss of sensibility to pain in Case 11 .

The area marked in black corresponds to the cutaneous analgesia. At various points are placed numbers corresponding to the pressure which caused pain. Thus 5.5 means that pain was caused by an algometer pressure of 5.5 kilograms. But such a number as $20=0$ means that no pain could be caused even by a pressure of 20 kilograms.

the arm was moved. But gradually the pain subsided, and in September, 1906, movements produced no pain, although the joint was still swollen and measured $7 \mathrm{~cm}$. more in circumference than the left shoulder.

Sensation.-(a) Symptomatic changes in sensation.-He bas complained throughout of no abnormal sensation, except of pain in connection with the right shoulder-joint. This was aggravated by movement and relieved by keeping the arm at rest; although particularly severe during the hot summer of 1906, it bad entirely disappeared hy September of the same year. 
(b) Sensibility to pain-(1) Analgesia.-When first seen, he was insensitive to the pain of a prick over the whole of the right upper limb, almost as high as the acromion. The cutaneous analgesia extended over the scapula behind, and over the third rib and third interspace in front.

This area has gradually increased under observation, and the extent of the cutaneous analgesia at the end of 1906 is represented on Fig. 55.

Throughout, the extent of the insensibility to the painful interrupted current has corresponded exactly with that to the pain of a prick.

In 1906, his sensibility to the pain of pressure was measured with the algometer, and the following readings were obtained :-

R.

\begin{tabular}{|c|c|c|c|c|c|c|}
\hline Thenar eminence & - & .. Pain at 5.5 & -. & & .. & . Pain at 4. \\
\hline Jentre of palm & $\therefore$ & .. Pain at 9 & . & - & . & 4 \\
\hline $\begin{array}{l}\text { Jlnar palm over fift } \\
\text { carpal }\end{array}$ & heta- & Pain at 5 & .. & $\cdots$ & $\cdots$ & . Pain at 4 \\
\hline Dorsum of hand :- & & & & & & \\
\hline Over second meta & carpal & . Slight pain & at 10 & . & $\cdots$ & . Pain at 3 . \\
\hline Over third metac & arpal & .. Just painful & at 11 & . & .. & $\ldots \mathrm{Pe}$ \\
\hline Over fifth metaca & rpal & . Pain at 8 & .. & .. & .. & $\ldots \mathrm{Pa}$ \\
\hline nterior aspect of w & rist & .. Pain at 9 & .. & .. & $\cdots$ & .. Pain at $2 \cdot 5$ \\
\hline orearm :- & & & & & & \\
\hline aspect & .. & .. No $\mathrm{p}$ & & $\cdots$ & . & $\ldots \mathbf{P a}$ \\
\hline Extens & .. & .. No ps & & .. & .. & \\
\hline Irm:- & & & & & & \\
\hline aspect & .. & .. No & & . & & .. I \\
\hline Inr & . & . No p & & .. & $\cdots$ & .. Pai \\
\hline joint & .. & . Deep & lges & (vide & p. 702) & ..Pair \\
\hline eramion $\quad$. & . & . Pain at 4 & . & . & .. & .. Pair \\
\hline clavicular & & $2 \cdot 5$ & . & . & $\cdots$ & .. Pain at \\
\hline Mastoid & .. & .. Pain at 1.5 & . & $\cdots$ & .. & .. Pain at 1.5. \\
\hline emple & . & .. Pain at 1.5 & $\cdots$ & $\cdots$ & $\cdots$ & .. Pain at 1.5 \\
\hline liac crest & .. & .. Pai & .. & . & . & .. Pain at 2. \\
\hline Sole $\quad \ldots$ & .. & at 2 & . & . & .. & .. Pain at 2 . \\
\hline
\end{tabular}

The results of these observations have been transferred to Fig. 55. The area marked in black is that which was insensitive to all cutaneous painful stimuli, and the numbers represent the pressure in kilograms which caused pain. It will be seen that over both the extensor and flexor aspect of the forearms and over the arm, pressure even up to 15 kilograms produced no pain. But no part of the hand was insensitive to this stimulus, although greater pressure was required to cause pain than over the normal hand.

(2) Deep hyperalgesia. - When first admitted in 1903, he 
suffered intensely from pain in and around the right shoulderjoint. But, in addition to this pain, he was found to be tender to deep touch over an area which occupied, on the outer aspect of the arm, the whole of the region of the deltoid to a point somewhat distal to the insertion of that muscle. When touched with the head of a pin, or when the skin was pinched between the finger and thumb, this area was exquisitely tender, and by means of either of these manipulations, its limits could be mapped out with considerable accuracy.

There was no tenderness to light touches, but pain was pro.

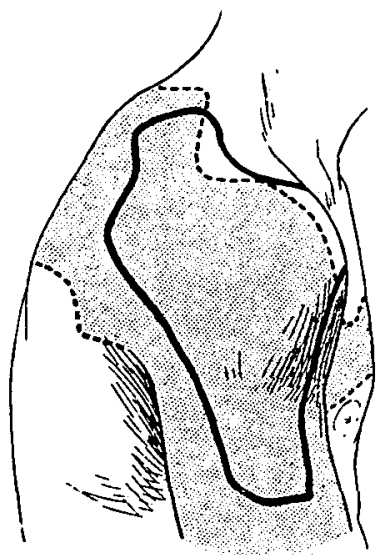

Frg. 56.

To show the relation of the deep hyperalgesia to the cutaneous analgesia in Case 11. The area of cutaneous analgesia is coloured grey and enclosed by a broken line; the area hyperalgesic even to the lightest pressure is enclosed by a heavy black line.

duced if the skin was tapped with cotton wool rolled into a hard wisp.

Yet the whole of this area, as far as it lay over the arm, was absolutely insensitive to the pain of a prick and to the painful interrupted current.

These observations were made and repeatedly confirmed during the first four months of 1904. Throughont the whole of this period the shoulder-joint was unusually painful.

A year later, in March, 1905, the joint was less painful and the area of tenderness less extensive. It then lay entirely within the limits of the cutaneous analgesia. If he was pricked within the 
limits of this tenderness, he complained of an "acute sharp pain" which persisted for about a minute after the stimulus had ceased. But, when the skin over the same parts was gently lifted between the finger and thumb, the prick of a pin over the lifted skin was
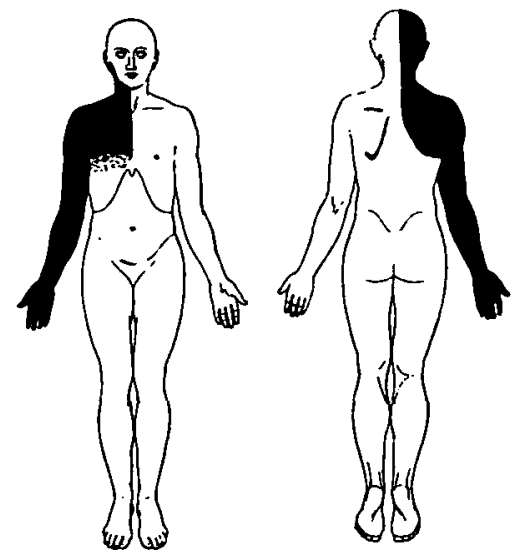

FIG. 57.

To show the loss of sensation to heat and cold in Case 11. The extent of the loss to heat on the face was occasionally somewhat greater than that of the loss to cold [vide Fig. 58].
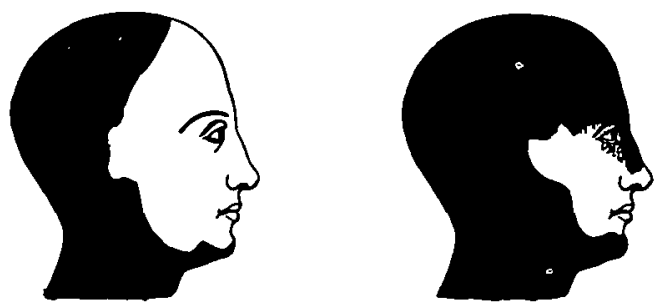

FIG. 58.

The extent of the loss to heat in Case 11 usually corresponded closely to that of the loss to cold. But at times the area insensitive to heat exceeded in extent that insensitive to cold, as shown in this Fig.

said to be a touch only; as soon as it was released, the prick was again said to cause pain. Any pressure over this area caused an "aching feeling."

In September, 1906, the joint was still swollen but no longer painful. All widespread hyperalgesia had disappeared. But, when tested with the algometer, he complained that any pressure pro- 
duced pain. "Something seemed to shift in the joint which immediately caused pain"; yet the pressure was below 1 kilogram, and could not be registered on the instrument.

Here we were face to face with a true deep hyperalgesia, co-existing beneath an area of complete cutaneous analgesia. The lightest pressure stimuli caused pain and the extent of the hyperalgesia could be marked out on the skin. Yet the superficial layers of the skin were analgesic to the prick of a pin when raised from the subcutaneous structures.

As the cutaneous analgesia gradually advanced up the neck, the joint became less painful and the area of excessive tenderness disappeared.

(c) Thermal sensibility. - When he was first seen, the loss of sensibility to cold had not invaded the scalp, but occupied the right half of the neck only. Gradually, it assumed the form shown on Fig. 57.

The extent of the loss to heat usually corresponded closely to that of the loss to cold. But at times the two forms of sensibility. were dissociated, as seen on Fig. 58, where the anæsthesia to heat exceeded that to cold. Over this dissociated area, he was carefully tested with temperatures of $25^{\circ} \mathrm{C}$, $27^{\circ} \mathrm{C}$., $35^{\circ} \mathrm{C}$., and $40^{\circ} \mathrm{C}$.; all were said to produce a sensation of touch only. None of these borders were at this time absolutely fixed, and the figures are given to illustrate the complete dissociation between the sensibility to heat and to cold, occurring over a part so sensitive to these stimuli as the forehead.

(d) Tactile sensibility. - Light touches were everywhere appreciated and there was no difference between the two upper limbs on careful testing with von Frey's hairs.

(e) Acucesthesia.-He could distinguish the head from the point of the pin everywhere over the analgesic area of the right upper limb.

(f) Appreciation of the difference in size was tested by means of metal rods with diameters of $2 \mathrm{~cm} ., 0.5 \mathrm{~cm}$., and $0.2 \mathrm{~cm}$. His answers were equally accurate over both upper limbs.

(g) The vibrations of a tuning fork (128) were appreciated everywhere equally on the two upper limbs. He was extremely quick in answering to a careful series of control experiments, which consisted of suddenly and unexpectedly damping the vibrations.

The legs were in every way normal.

(h) The sense of passive position and movement was everywhere preserved, even for movements of the grossly disorganised shoulder-joint. 
(i) The compass-test.-Throughout, we have been unable to discover any difference in the compass-records obtained from the two upper limbs. His threshold (transverse) for the palms lies between $1 \mathrm{~cm}$. and $2 \mathrm{~cm}$; at $2 \mathrm{~cm}$. he has always answered correctly, and occasionally he has given a perfect series of answers at $1.5 \mathrm{~cm}$. over both palms; but at $1 \mathrm{~cm}$. he has uniformly failed to a varying degree.

(j) Tactile localisation was equally good over both arms and both legs.

Cranial nerves.-The pupils were equal, and reacted well to light and accommodation. The movements of the face, tongue, palate and vocal cords were perfectly performed.

Sphincters.- He had no trouble with the sphincters at any time.

Spine.-When first seen, his spine was straight ; but by April, 1905, a slight degree of scoliosis had appeared, the concavity of the curve lying to the right, in the middle of the thoracic region.

\section{Case 12.- Walter John H.}

First cxamined in March, 1904. For eight years before this date he had been unable to appreciate heat and cold applied to the right hand, or to feel the pain of a suppurating finger. For one year the right half of the face had sweated cxcessively when he ate or drank.

At this time there were no changes of any kind pointing to disease of the nervous system, except the loss of sensation to painful or thermal stimulation shown on Figs. 59 and 60. His condition remained unchanged until September, 1905.

By August, 1906, the muscles of the right hand had become wasted and the hand had lost power. The loss of sensation had greatly increased, and had invaded the left hand (Figs. 61 and 62). Sensibility to light touch and pressure, and the sense of passive position and movement, were unaffected.

Walter John H. was first examined in March, 1904. He was then 30 years of age and a fishmonger hy trade.

$\mathrm{He}$ complained that for the last eight years or more he had noticed that the right arm was different from the left; it seemed colder and there was a sensation of tightness in his fingers. When he took a hot thing into his right band, he did not at once recognise it was hot. In 1903 , he poisoned the thumb of the 
right hand ; it "gathered" and suppurated, but he experienced no throbbing or pain, as with other fingers under similar circumstances.

For the last twelve months, it happened that whenever he ate or even at times when he drank, the whole right half of his face, forehead and head sweated profusely. He has been compelled to use a bandkerchief to prevent the sweat running off his face.

Otherwise, he complained of no abnormal symptoms and said he was in the best of health.

He had suffered twice from gonorrhœa ten years before, but neither attack was followed by any symptoms pointing to syphilis. $\mathrm{He}$ was married, and had two bealthy children.

His father died at the age of 47 of dementia paralytica, and his mother is highly neurotic; she has had several attacks of hysteria in which she has lost her speech. He has nine living brothers and sisters, all of whom are healthy. No collateral history of neuroses could be obtained.

From 1904 until April, 1905, the signs and symptoms of his disease remained unchanged, and we shall therefore give first the results of our examination in April, 1905.

\section{Condition in April, 1905.}

He was a largely built man, unduly corpulent for his age, but otherwise of healthy appearance. His pulse was regular, of good tension, and the arterial wall was not thickened. Heart and lungs were unaffected, and the urine was normal.

From the point of view of the nervous system, the only change was in sensation; there was no paralysis or wasting of muscles, no trophic changes in the skin or nails. His knee-jerks were equal and normal, ankle-clonus was not obtained, and both plantar reflexes gave a flexor response. The pupils reacted to light and accommodation. Vision was good; hearing, taste and smell were unaffected. No abnormality was found in the movements of the face, the tongue, the palate or the larynx. Both sphincters acted normally.

Sensation.-About 1896 he began to notice that whenever he had a cough a stabbing pain struck him in the right shoulder blade over a point at the vertebral border of the spine of the scapula. The centre of incidence of this pain gradually shifted upwards. At first it was situated close to the spine of the scapula; then it moved to the back of the neck, thence to the parietal region. From this point it moved forwards to the vertex, then to the temple, and in April, 1905, was situated 
in the right eyeball. Each time he coughed he had a feeling as if he had been struck a violent blow in the eye.
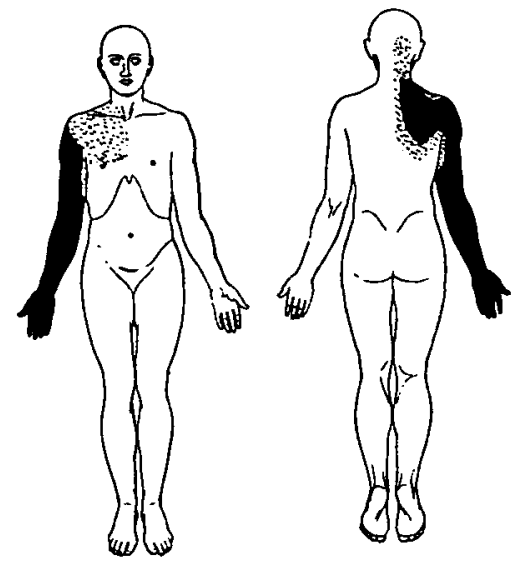

Fig. 59.

To show the loss of sensibility to cutaneous painful stimuli in 1905 .

He complained of no abnormal sensations in the right arm and hand, except that in the winter they did not suffer from the cold weather in the same way as the left.
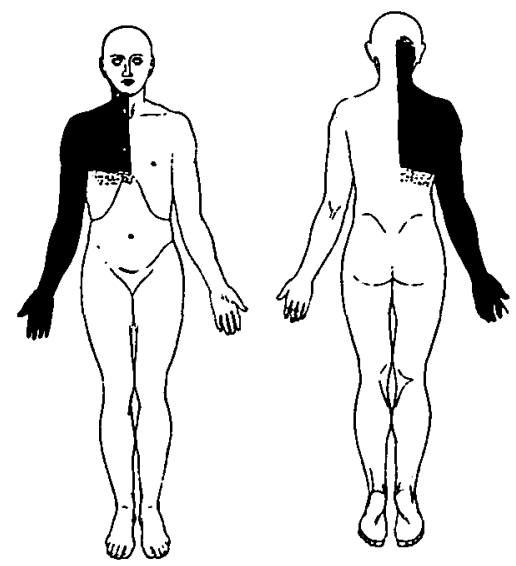

Fig. 60.

To show the loss of sensation to heat and cold in 1905 .

Cutaneous painful stimuli were not appreciated when applied anywhere within the heavily-shaded area of Fig. 59. Over the parts dotted in this figure, a prick sometimes caused pain, but was frequently said to be a touch only. 
He was entirely insensitive to all degrees of heat and cold over the darkened parts of Fig. 60. The border on the face and behind the ear was unusually definite.

The stimuli of light touch, deep touch and pressure were everywhere appreciated and accurately localised. He could tell the bead from the point of a pin, even over parts that were completely analgesic. In the same way, he was able to discriminate everywhere between contact with a circular metal surface $1 \mathrm{~cm}$. in diameter and a similar surface with a diameter of $2.5 \mathrm{~cm}$. There was no loss of the sense of passive position or movement. The vibrations of a tuning-fork (128) were everywhere recognised. The two points of the compasses gave the same result on both the normal and abnormal hand: his answers were good, when the two points were separated for a distance of $1 \mathrm{~cm}$., and applied transversely.

\section{Condition in 1906.}

In August, 1906, his condition had undergone a remarkable change. During the previous winter, he had noticed a gradual loss of power in the right arm, and the grip of the right band had become less powerful. The little finger became gradually abducted from its fellows, when the band was at rest. He also noticed involuntary twitchings in the muscles of the thumb and of the forearm.

He stated that in September, 1905, he experienced a sudden acute pain in the left arm, and since then had suffered from cramp in the left hand. These symptoms were, he believed, the same as those which preceded the loss of power in the right hand.

Motion.-The thenar and hypothenar eminences and the muscles in the first interosseous space of the right hand were definitely wasted. Fibrillary twitchings could be seen in the muscles of the right thumb, and in those which arise from the external condyle of the humerus.

The grip of the right hand was weaker than that of the left, and he had difficulty in opposing the thumb. All the interosseous movements were well performed.

The small thumb-muscles reacted slowly to the interrupted current, and, when the right abductor minimi digiti was stimu. lated, a certain number of fibrils only contracted, in contrast to the normal reaction of the synonymous muscles in the other hand. All the muscles reacted well to the constant current, except the 
thenar group of the right hand, which reacted as easily with the anode as with the kathode.

The muscles of the left hand and arm were entirely unaffected.

Sensation.-A remarkable increase had occurred in the extent of the loss of sensation to painful and thermal stimulation (as shown on Figs. 61 and 62). The limits of the loss of sensation to the painful interrupted current corresponded exactly with those of the analgesia to prick. Over the whole of the thermo-anæsthetic area on the right arm, he failed to recognise heat and cold with any degree of temperature between melting ice and $60^{\circ} \mathrm{C}$. Over the left arm the results were less definite, and he sometimes

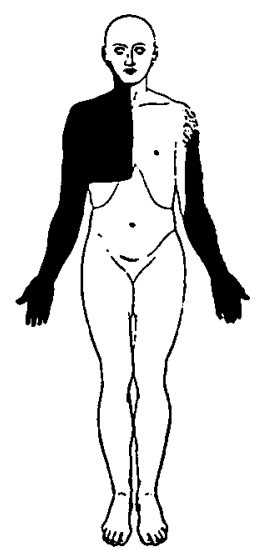

FIG. 61.
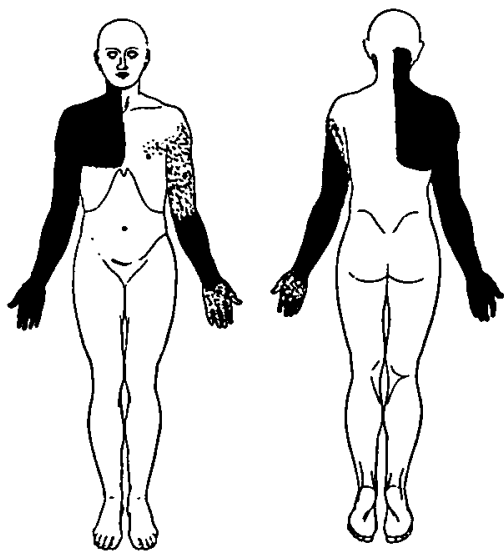

Fig. 62.

To show the loss of sensation to cutaneous painful stimuli (Fig. 61) and to heat and cold (Fig. 62) in 1906. Evidently the loss of sensation was extending in the left upper extremity.

seemed to appreciate stimulation by the more extreme degrees of heat and cold, where water at $40^{\circ}$ or $20^{\circ} \mathrm{C}$. produced no sensation. Evidently, the loss of sensibility to heat and cold was here less complete than in the right arm.

The border on the face between parts that were sensitive and those that were insensitive to thermal stimuli was remarkably constant and varied little, according to the direction in which the tests were made. In 1905 the analgesia did not extend on to the face, as will be seen from Fig. 59. But, in 1906 the loss of sensation to painful stimuli spread until the residual sensibility was identical to prick, to heat, and to cold. Moreover, the border so marked out corresponded exactly with that deter- 
mined by means of thermal stimuli nearly a year and a half before. This border is shown on Fig. 31, p. 612.

The algometer gave the following readings, which showed that deep pressure caused no pain over the whole of the right upper extremity. Over the left palm and flexor surface of the forearm, pain was not produced at first until the pressure was raised considerably above the normal threshold. But on repeating the experiment pain was produced by pressures no greater than those which are painful on a normal hand.

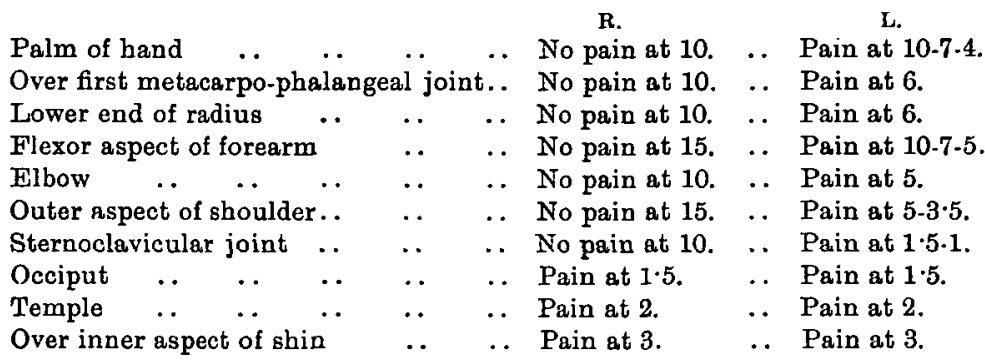

The vibrations of the tuning-fork (128) were less clearly recognised over the whole of the right arm from the elbow to the shoulder-joint than over similar parts of the left side.

Stimulation with cotton wool and with pressure was everywhere appreciated, and the point of contact accurately localised.

No. 5 of von Frey's hairs ( $360 \mathrm{mgm}$.) produced a sensation of touch over both palms and the flexor aspect of both forearms.

The sense of passive movement and position was entirely unaffected, and there was no difference in the sensibility to the compass test between the two upper limbs. Everywhere, over both arms and both hands, he could tell the point from the head of a pin, even over parts that were insensitive to a prick, and he could appreciate the difference in size between metal discs of $2 \mathrm{~cm}$., $1.4 \mathrm{~cm}$., $75 \mathrm{~cm} ., \cdot 2 \mathrm{~cm}$; but he was somewhat less accurate on the right side than the left.

\section{Case 13.-Thomas B.}

For three years, and probably longer, he had suffered from chronic disease of the spinal cord (syringomyelia).

Analgesia and thermo-ancesthesia existed over the areas in Fig. 63.

No other forms of sensation were affected.

The muscles of both hands, especially the right, were wasted.

The left elbow joint was disorganised.

The reflexes were unaffected. 
Thomas B., aged 48, a labourer in a jam factory, came to the London Hospital on November 18, 1905, complaining of a painful swelling of the left elbow.

He dated his illness from a fall on to his head three years before. But, for more than three years, his hands had always "felt cold," even when he was sitting before a fire ; and when he went to bed, it was more than an hour before they became warm.

During the last two years before he came to the hospital, he had noticed wasting of the muscles of his hands, first in the right and later in the left.

He had been in the Army, serving five years in India; there he had contracted syphilis, for which he was treated for a month only. Otherwise, his health had been excellent. He had been married for twenty-four years and his wife had had thirteen children.

\section{Present Condition.}

He has been under observation for over twelve months and his condition has changed little, if at all. $\mathrm{He}$ is a short but sturdily built man of fair intelligence. He was firm to controltests, and, provided the experiments were not too complex, gave consistently accurate answers.

$\mathrm{He}$ was in every way normal, except for the following changes in the nervous system.

Motion.-The small muscles of the right hand were much wasted, the thenar and hypothenar eminences were flattened, and the thuinb was extended, lying in the plane of the palm. All the interossei showed signs of atrophy. The proximal phalanges of the fingers were hyper-extended at the metacarpo-phalangeal joints, and the two terminal phalanges were flexed. No con. traction could be seen or felt when he attempted to abduct or oppose the thumb; interosseous movements were impossible, and he could not abduct the index or little finger.

All movements of the wrist could be performed with ease: and the fore-arm muscles showed no signs of wasting.

The left band was wasted, but to a less degree than the right, and the thenar eminence was flattened.

He could carry out all movements with his left hand and could even oppose and abduct his thumb.

None of the muscles of the left fore-arm were wasted, and all acted perfectly.

All the muscles of both upper extremities reacted well to the interrupted and to the constant current, excepting only the outer 
thenar group and the interossei of the right hand; these muscles could not be made to react, even to a strong interrupted current. On stimulation with the constant current, they reacted slightly, more easily to the cathode than to the anode.

His gait was normal and he could stand steadily even with his eyes closed.

The muscles of the legs showed no signs of wasting or spasticity.

Reflexes.-The knee-jerks were in every way normal: no ankle-clonus could be obtained and the plantar reflex gave a flexor response on both sides.

Articular changes.-The left elbow was much swollen and dis-

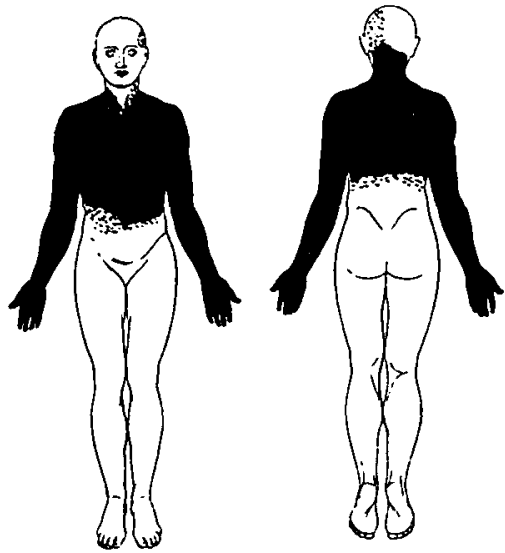

F'IG. 63.

To show the loss of sensation to cutaneous painful stimuli and to hest in Case 13. The loss of sensation to cold is described in the text.

organised ; creaking could be obtained on manipulation, and there was excess of fluid with much bony outgrowth around the joint. A radiograph showed much destruction of the opposing surfaces of the elbow-joint, with the formation of many osteophytic masses.

He complained of no other joint in his body, but a radiograph of the right elbow also showed slight bony changes.

Spinal column.-The spine showed a distinct lateral curve in the thoracic region with a convexity to the right, and a second curve towards the left in the region of the lower thoracic and lumbar spines.

Sensation.-He occasionally complained of pain in the left 
elbow, probably induced by the nature of his work; for, owing to the loss of power in the right hand, he was compelled to use the left arm to raise heavy bags of sugar by means of a hand-winch. At no time could we elicit pain by manipulation of this joint.

The curious sensations of coldness in the hands, so marked a feature during the winter, greatly improved with the coming of summer. On an extremely hot day in September, 1906, these sensations had entirely disappeared.

(a) Sensibility to painful stimuli.-He was entirely analgesic to the prick of a pin and to the painful interrupted current over the area shown in Fig. 63 ; this was of somewhat greater extent in the winter than in the summer. But the borders of the loss of sensation always merged gradually into parts of normal sensibility.

The pain of deep pressure, as tested by the algometer, was completely lost over both upper extremities and over the upper part of the trunk. Elsewhere, pain was produced as usual, as will be seen from the following records. At each application he was able to recognise the gradually increasing pressure; but over the analgesic areas it caused no pain.

\begin{tabular}{|c|c|c|c|c|c|c|c|c|}
\hline \multirow{2}{*}{\multicolumn{2}{|c|}{$\begin{array}{l}\text { Palm of hand } \\
\text { Thenar eminence }\end{array}$}} & \multirow{2}{*}{$\begin{array}{l}\cdots \\
\cdots\end{array}$} & \multirow{2}{*}{$\begin{array}{l}\cdots \\
\ldots\end{array}$} & & \multicolumn{3}{|c|}{ R. } & $\begin{array}{c}\text { L. } \\
\text { No pain at } 15 .\end{array}$ \\
\hline & & & & .. & .. & No pain at 15 & . & No pain at 15 . \\
\hline Elbow & . & . & . & . & .. & No pain at 15 & . & No pain at 15. \\
\hline Shoulder & . & .. & . & . & .. & No pain at 15 & . & No pain at 15 . \\
\hline Sternoclav & cular jc & jint & .. & .. & .. & No pain at 10 & .. & No pain at 10. \\
\hline Mastoid p & cess & & $\ldots$ & . & .. & Pain at 3 & .. & Pain at 3. \\
\hline Temple & .. & .. & . & $\ldots$ & $\cdots$ & Pain at 2 & . & Pain at 2. \\
\hline Sole of fo & & . & . & . & . & Pain at 3 & . & Pain at 4. \\
\hline Inner aspe & i ti & & .. & . & .. & Pain at 3.5 & .. & Pain at 3.5 . \\
\hline Outer aspe & of ant & erior & peri & liac & & Pain at 5 & .. & Pain at 5. \\
\hline
\end{tabular}

(b) Sensibility to thermal stimuli.-During the winter, the thermo-anæsthesia corresponded almost completely with the extent of the cutaneous insensibility to pain; but during the summer of 1906, it was found that, although the insensibility to heat still corresponded with the loss of sensation to prick, the anæsthesia to ice was of somewhat smaller extent. A long series of experiments were made at this time over the outer aspect of the arms, where he seemed to be sensitive to ice, though insensitive to heat, and it was found that he was able to appreciate temperatures from $0^{\circ}$ to $27^{\circ} \mathrm{C}$., calling them all cold; but above this temperature, he seemed to be unable to appreciate certainly any thermal stimulus, calling $70^{\circ} \mathrm{C}$. " a shade warm."

(c) Tactile sensibility.-The palms of both hands were horny voL. Xxix. 
and insensitive ; but, except over the hairless parts of the hands, he was normally sensitive everywhere to stimulations with cotton wool. Sensibility to pressure was nowhere disturbed.

(d) Acucesthesia and discrimination of difference in size.- $\mathrm{He}$ could distinguish accurately the bead from the point of a pin all over the analgesic area. Neither caused pain, but he described a prick as "like the point of a needle"; the head of the pin was said to be "blunter."

To test his power of recognising differences in size, steel rods were used with diameters of $2 \mathrm{~cm}$., $0.4 \mathrm{~cm}$. and $0.2 \mathrm{~cm}$. His answers were invariably correct even over both palms.

(e) Passive position and movement.-To this test his answers were remarkably quick and accurate; even movements of the disorganised elbow were correctly appreciated.

When any muscle of one hand was thrown into action by stimulation with the interrupted current, he recognised the movement, imitating it perfectly with the opposite hand. Similarly, he could reproduce with the one upper extremity every movement produced in the other by electrical stimulation.

( $f$ ) The compass-test.-Over both palms the threshold to the compasses applied transversely lay between 2 and $3 \mathrm{~cm}$. When applied longitudinally, he gave a perfect series of answers at $2.5 \mathrm{~cm}$.

(g) Tactile localisation was nowhere affected, and we could find no difference between the two sides of the body.

Sphincters.-Micturition and defæcation were unaffected.

\section{Case 14.-Frorence R.}

A case of syringomyelia, watched from 1899 to the present time. The first signs consisted of wasting of the small muscles of the left hand with disturbed sensibility, which was transitory only.

Gradually, the muscular wasting increased, and in 1902 sensibility was again found to be disturbed in the left hand. This change progressed until, at the end of 1906, the cutaneous insensibility to pain was complete everywhere and the thermo-anasthesia was widespread.

No other form of sensibility has been disturbed at any time.

The reflexes and sphincters have remained unaffected.

Florence R. has been under observation since October, 1899 when she first came to the London Hospital, complaining of neuralgic pains in the occipital region and numbness in the left arm. For several months she had noticed a tendency to drop 
things from her left hand but, until the muscular atrophy was pointed out to her at the hospital, she was not aware that the hand was wasted.

A stout, healthy looking woman of 24 , she showed no abnormal signs except those pointing to disease of the nervous system.

The small muscles of the left hand were wasted, especially the interossei. The hand had begun to assume the "clawed" position, but the thumb lay in the normal position, and the thenar muscles, though flabby, were not definitely wasted.

At this time the only other abnormal sign consisted of a diminished sensibility to heat and cold over a small area on the forearm and hand (Fig. 64).

By January, 1900, the muscular atrophy in the left hand had
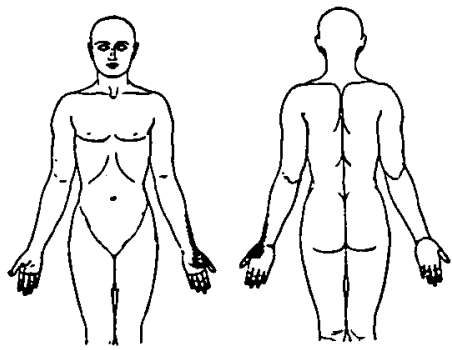

Fig. 64.
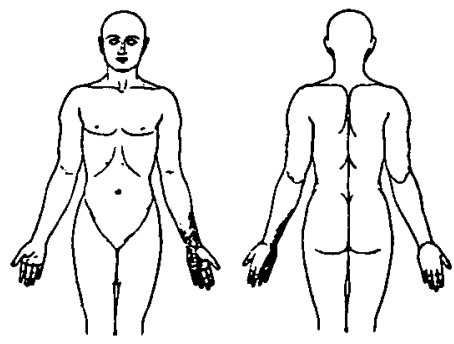

Fig. 65.

Fig. 64 shows the disturbance of thermal sensibility discovered in October, 1899. Sensation to prick was unaffected.

Fig. 65 shows the disturbance of sensibility to heat, to cold, and to prick, which appeared in March, 1902. For the intervening two and a half years no definite loss of sensation could be discovercd in spite of the progressive muscular atrophy.

greatly increased, and the muscles of the left forearm showed signs of wasting, but no sensory disturbance could be discovered.

In March, 1900, the interossei, thenar and hypothenar muscles of the left hand were much wasted, and she could not oppose, abduct or adduct the thumb. The hand was " clawed," with the tingers over-extended at the metacarpo-phalangeal and flexed at the phalangeal joints. At this time the muscles of the right hand had begun to waste, more particularly the first dorsal interosseous and the muscles of the thenar eminence. The only muscles of either extremity which did not react normally to electrical stimulation were the interossei and the abductor and opponens pollicis of the left hand; none of these muscles responded to the interrupted current, but they contracted slowly to making the constant current more readily with the anode than with the cathode. 
Throughout the first two and a half years, no definite change could be discovered in sensation except during October, 1899, when she first came to the hospital. But in March, 1902, after another severe attack of neuralgic pains, distinct loss of sensation to heat and cold and to prick was found over the area shown on Fig. 65. The muscular wasting had steadily progressed, not only in the left hand, but also in the small muscles of the right hand; the left forearm had already become affected.

In November, 1902, she suffered greatly from pain up the back of ber neck and in both shoulder-blades, and was so unwell sbe was compelled to give up her employment. The loss of sensation advanced greatly in extent. Over the areas shown on Fig. 66, she was insensitive to stimulation with all degrees of cold; the loss of sensation to heat was somewhat more extensive on the left upper

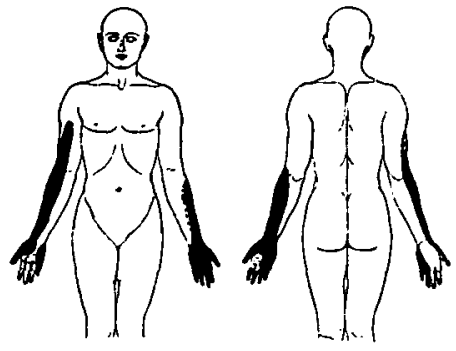

Fig. 66.
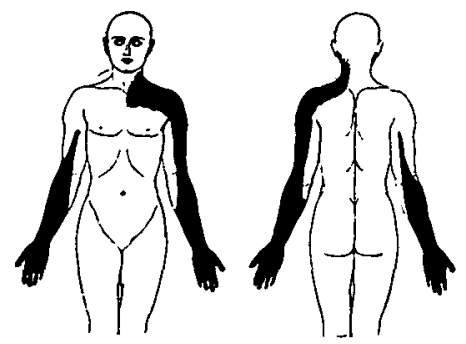

Fig. 67.

Fig. 66 shows the areas insensitive to cold in November, 1902.

Fig. 67 shows the areas insensitive to cold in June, 1903. The extent of the loss to heat and to prick is given in the text.

extremity, somewhat less extensive on the right. Sensibility to prick was nowhere lost, but was undoubtedly diminished over the left hand. Every other form of sensation was perfectly preserved.

The muscular wasting had made little or no progress, and the electrical reactions were identical with those obtained in March, 1900.

By June, 1903, the loss of sensation had progressed greatly. The greater part of the left arm and the whole of the right forearm and hand were insensitive to all degrees of cold (Fig. 67).

The extent of the loss of sensation to heat corresponded on the left arm with that to cold, but was less extensive over the right upper extremity. Analgesia to prick was almost confined to the left half of the neck and left upper extremity, where it corresponded exactly with the area on Fig. 67. Every other form of sensibility was perfectly preserved. 
The muscular wasting had progressed very little, if at all, and the reflexes were unaffected.

The loss of sensation spread rapidly, and by November, 1903, had invaded the face and greater part of the scalp on the left side. Over the whole left half of the body, the loss of sensation was coterminous to prick, to heat and to cold (Fig. 68). But on the right side, although the thermo-anæsthesia occupied a considerable portion of the right upper extremity, analgesia to prick was found over the hand and part of the forearm only.

The muscles originally affected had continued to waste, but no further ones had been attacked since March, 1902. The thenar muscles and abductor minimi digiti of the left hand did not react to the interrupted current, but a response could be obtained from all the interossei in spite of their profound wasting. Every muscle of the left hand reacted to the constant current, even
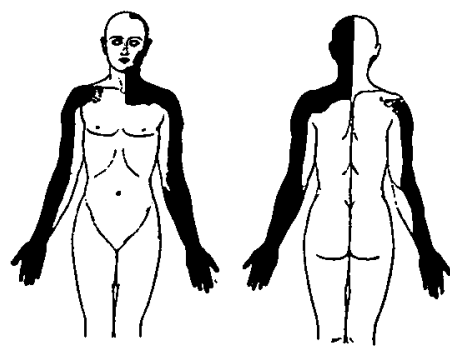

Fig. 68.
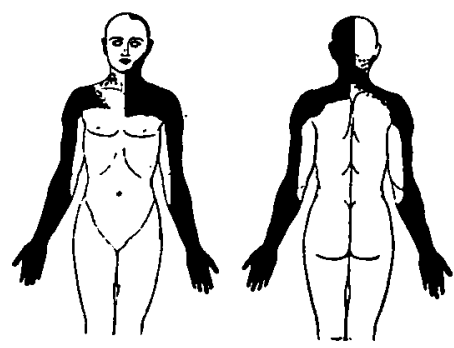

Fig. 69.

Fig. 68 shows the loss to sensation to cold and to heat in November, 1903. 1904.

Fig. 69 shows how little the loss of sensation had advanced by February,

the wasted strips which represented the muscles of the thumb. In the right hand, normal reactions were everywhere obtained.

From November, 1903, to February, 1904, the loss of sensation did not extend on the left half of the body and head, but slight advance took place over the right shoulder and neck.

During this period, advantage was taken of the stationary condition of the symptoms to work out the extent of residual sensibility on the left half of the face (Fig. 33, p. 612). The border between normal and abnormal parts was found to differ little, whether the stimulus was a prick, or test-tube containing water at different temperatures.

By February, 1905, the loss of sensation to all three stimuli had extended considerably on the trunk, and the thermo-anæsthesia exceeded somewhat the loss to prick, and was no longer bounded by definite borders (Fig. 70). 
The muscular wasting was at this time profound in all the small muscles of both hands, but greater in the left than the right. She could not make any movement with the small muscles of the left thumb, and adduction and abduction of the fingers was impossible. All movements of the right hand could be performed, but those requiring contraction of the intrinsic muscles were feeble. The only muscles which did not react to the interrupted current were those of the left thumb.

\section{Condition from May to September, 1906.}

She was a short, red-cheeked woman, of healthy appearance, except for her wasted hands.

Her speech is unaffected and she has never suffered from fits or sudden attacks of weakness in any limb.

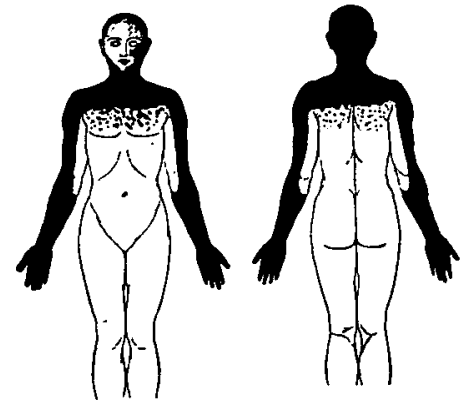

Fig. 70.
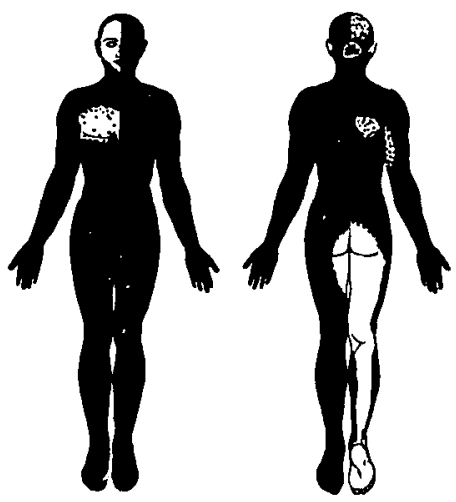

Fig. 71.

Fig. 70 shows the thermo-anæsthesia in March, 1905.

Fig. 71 shows the loss of sensation to cold in September, 1906.

Sensation.-She has suffered from much pain in the head, beginning in the left temple and passing over the forehead and bridge of the nose. The pain then passed into the spine, extending as low as the lumbar region.

Not uncommonly she experienced a curious sensation in the left half of the face, exactly as if it had been rubbed with menthol. Occasionally she has felt as if she had no left ear, and is compelled to touch it to assure herself of its presence.

During the extremely hot weather she was comfortably cool.

(a) Sensibility to pain.-The cutaneous analgesia had advanced 
to such an extent, that there was no part of the body or face where she could appreciate the prick of a pin, or the painful aspect of an interrupted current.

Over both upper extremities and over both thighs no pain could be produced by excessive pressure, and the following records were obtained on the algometer :-

R.

\begin{tabular}{|c|c|c|c|c|c|c|c|c|}
\hline & & & & & & T To & & in at 90 \\
\hline Palm & . & . & $\cdots$ & $\cdots$ & .. & No pain at 20 & .. & \\
\hline Porearm & .. & .. & $\cdots$ & $\cdots$ & .. & No pain at 15 & .. & No pain at 15 . \\
\hline Arm & . & . & . & .. & .. & No pain at 15 & .. & No pain at 15 . \\
\hline Shoulder & . & $\cdots$ & . & .. & .. & No & .. & \\
\hline ternoclav & vicular & joint & .. & .. & .. & it pain at 5 & . & \\
\hline emple & .. & .. & .. & .. & .. & ht pain at 5 & .. & Sli \\
\hline ole & . & . & . & $\ldots$ & .. & at 20 & & $n$ at 5. \\
\hline uter mal & lleolus & & .. & .. & . & $\mathrm{Pa}$ & & Pain at 4. \\
\hline ane & & shin & .. & .. & .. & $\mathrm{Pai}$ & .. & Pain at 3. \\
\hline 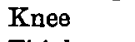 & . & .. & .. & .. & . & at 15 & . & No pain at \\
\hline & .. & . & .. & .. & .. & t 15 & .. & \\
\hline thom & ecto & & & & & No $\mathrm{p}$ & & \\
\hline
\end{tabular}

(b) Thermal sensibility.-She was insensitive to all degrees of cold stimulation over the areas shown on Fig. 71. But, over the parts which remain undarkened on this figure, she did not always respond, even to stimulation with ice. It was evident that the sensibility of these parts varied with her general condition.

To heat, especially the more extreme degrees, she was more widely sensitive; but it is extremely difficult to be certain how far this is based on a true appreciation of the nature of the stimulus, on account of her tendency to call even neutral touches "warm."

(c) Tactile sensibility. - Stimulation with cotton wool was everywhere appreciated, and she recognised No. 5 of von Frey's hairs over both palms.

(d) Acucesthesia.-She could tell the head from the point of a pin everywhere, in spite of the total cutaneous analgesia.

(e) The vibrations of a tuning-fork were quickly recognised everywhere.

$(f)$ Sense of passive position and movement was everywhere perfectly preserved.

(g) Tactile discrimination.-Over both palms the compassrecords were perfect at $2 \mathrm{~cm}$., when the points were applied longitudinally.

(h) Tactile localisation was good everywhere; a series of tests were made over the hands, using No. 5 of von Frey's hairs as the stimulus, and she named the spot touched with remarkable accuracy. 
$\therefore$ Motion. -The wasting and loss of power had not changed materially from the condition in 1905.

Reflexes.-The knee-jerks were normal. No ankle-clonus could be obtained and both plantar reflexes gave a flexor response.

Sphincters.-She had no trouble with micturition or defæcation.

Cranial nerves.-The pupils were equal and reacted normally. Movements of the face and tongue were well performed.

On ophthalmoscopic examination, disc and fundus were found to be normal.

Case 15.-George B.

A case of chronic intramedullary growth or syringomyelia.

When first seen by us in October, 1906, he complained that sensation had been altered in his right hand for seven years, and that he had gradually lost the power of writing.

Profound loss of sensation was present over the right upper extremity. The loss of tactile sensibility was unilateral (Figs. 75 and 76), but to painful and thermal stimuli the left upper extremity was also affected (Figs. 72, 73, and 74).

The sense of passive position was absent in the fingers and thumb of the right hand. Tactile localisation and tactile discrimination were unaffected over parts which were sensitive to touch.

Early signs of wasting and some weakness were present in the right hand. The right leg tended to be spastic.

The right knee-jerk was greatly increased, and the right plantar reflex gave an extensor response.

Rotatory nystagmus was present in both eyes when he looked to the right.

No other abnormal signs were found in the territory of the cranial nerves.

For some time he had had difficulty in passing water and in holding his motions when they were loose.

George B., aged 35, a painter, came to the London Hospital in October, 1906, for numbness and loss of power in the right band.

Seven years before, he began to notice that the hand seemed always cold. On many occasions he burnt himself and knew 
nothing of it until he saw the burns on his right hand. He could retain an object so long as he saw what he was holding, but often dropped it from his right hand, ignorant that it was no longer in his grasp. The power of writing had gradually left him.

Two years before he came to the hospital his right hand was poisoned by the bite of a mosquito, but the inflammation and even the incisions which became necessary on account of the swelling were entirely painless.

For eighteen months he had noticed stiffness of the right hip with some "numbness" of the sole of the right foot, and this leg had " jumped" at night.

$\mathrm{He}$ had noticed some difficulty in passing water for a considerable time before coming to the hospital, and for ten months had lost all sexual desire.

A bony projection had been slowly growing at the distal end of the proximal phalanx of the right thumb.

$\mathrm{He}$ was temperate and denied infection with syphilis or gonorrhœe. He bad been married for ten years; his wife had never been pregnant.

\section{Condition from October, 1900, to February, $190 \%$.}

He was a stout, heavily-built man, somewhat slow of speech, but intelligent, and able to concentrate his attention during the testing of sensation.

Except for the symptoms and signs produced by the disease of the nervous system, he was in every way normal.

Motion.-He walked on a somewhat broad base, but did not drag either foot, and could stand well with the eyes closed. He could not stand on either foot alone. When be attempted to stand on the left foot with the right raised, a few swaying movements preceded his fall, but when he raised the left foot he fell instantly.

The right leg was held stiffly and, if he was examined after he had been walking about, all the joints could be moved passively without undue effort. But the right leg was distinctly spastic after he had been for some time in bed, especially at the end of a morn. ing spent in sensory stimulation whereby the leg had been frequently thrown into spasmodic contraction. The right leg was not paralysed or wasted.

The condition of the left leg was in every way normal.

There was no wasting of either hand, but fibrillary twitching was noticed in the third and fourth interosseous spaces. The grasp of the right hand was somewhat more feeble than that of 
the left (right $16 \mathrm{kgm}$., left $23 \mathrm{kgm}$.), and the fingers were out of alignment, coming together unsteadily. This was especially noticeable in the little and ring fingers; but every movement of the right hand was possible. The extensors of the right wrist acted less powerfully than those of the left forearm; every other movement of both upper extremities was well and equally performed. All muscles, including those of the right hand, reacted normally to both forms of electrical stimulation.

Reflexes. - The right knee-jerk was greatly increased compared with the left. No ankle-clonus could be obtained, but the right plantar reflex gave an extensor response. That on the left side was of the normal flexor type.

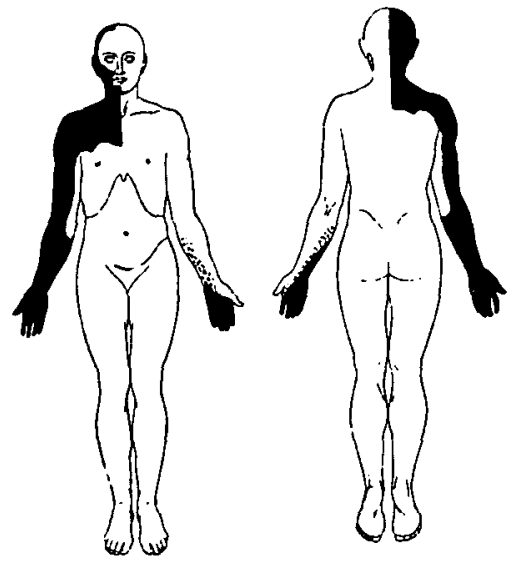

Frg. 72.

To show the loss of sensation to cutaneous painful stimuli in Case 15. The patch of remaining sensibility on the inner aspert of the right arm was worked out by testing from sensitive towards insensitive parts.

Sensation.-(a) Sensibility to painful stimuli.-The whole of the parts marked on Fig. 72 were insensitive to cutaneous painful stimuli. Over the inner aspect of the right arm, sensation was still present to prick and to the painful interrupted current, and the borders of this area in the long axis of the arm were sbarply defined.

The condition of the left hand and forearm varied from day to day, but at the best sensibility to cutaneous painful stimuli was gravely diminished, and on several occasions the greater part of the hand was entirely analgesic. 
The algometer gave the following remarkable results, which are the average records in kilograms of many examinations.

R.

L.

Palm of the hand _. Pain from 10 to 13 .. No pain with 15 .

Dorsum of the hand $\quad \ldots \quad$ Pain at $7 \quad \ldots \quad$. Pain at from 4 to 7 .

Flexor and extensor as- ? Pain usually absent at | Pain usually absent at pects of forearm $\int$ pressures below 13$\}$ pressures below 13.

Olecranon .. $\quad \ldots \quad \quad \ldots \quad$ No pain at $14 \quad \ldots \quad$.. Pain at 6.

Shoulder joint $\quad \ldots \quad \ldots \quad$ No pain at $13 \quad \ldots \quad$.. Pain at 6.

Clavicle $\ldots \quad \ldots \quad \ldots$ Pain at $8 \ldots \quad \ldots \quad \ldots$ Pain at 3.

Angle of jaw $\quad \ldots \quad \ldots$ Pain at $3 \ldots \quad \ldots \quad \ldots$ Pain at 2.

Temple $\quad . \quad \ldots \quad \ldots$ Pain at $1.5 \quad \ldots \quad$.. $\quad$ Pain at 1.5.
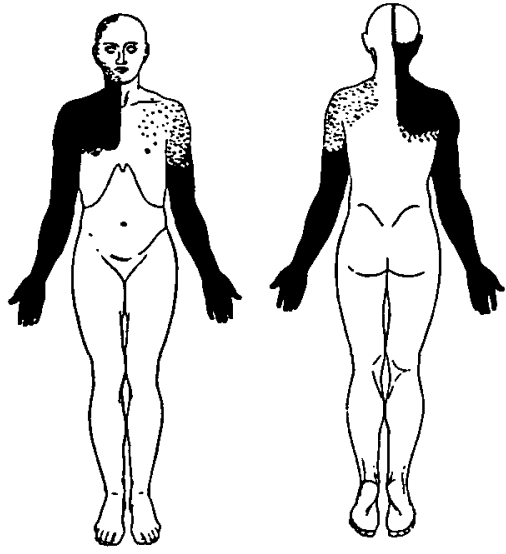

FIG. 73.

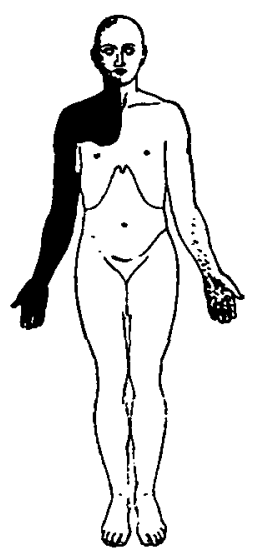

Fig. 74.

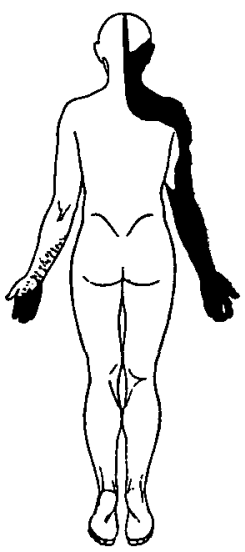

Fig. 73 shows the loss of sensation to heat in Case 15.

Fig. 74 shows the loss of sensation to cold in the same case. Here the patch of remaining sensibility on the inner aspect of the right arm was worked out by testing from sensitive towards insensitive parts.

Orer the remainder of the body there was no loss of sensibility to painful pressure, and the algometer gave equal readings on the two lower extremities.

R.

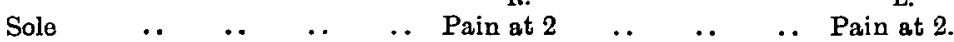

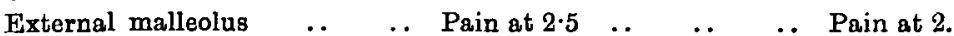

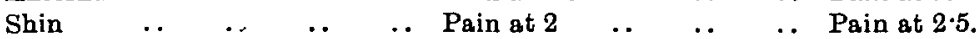

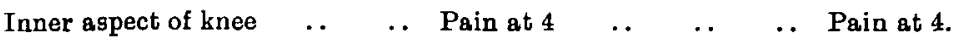

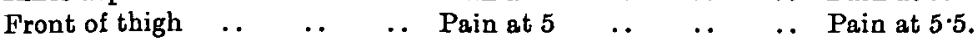

It is evident from these records that the loss of sensibility to the painful aspect of the pressure was less extensive than that of the cutaneous analgesia. Moreover, the right band still remained 
sensitive to painful pressure though to a diminished degree, in spite of the complete insensibility of the whole forearm and hand to cutaneous painful stimulation.

(b) Thermal sensibility. - $\mathrm{He}$ was insensitive to all degrees of

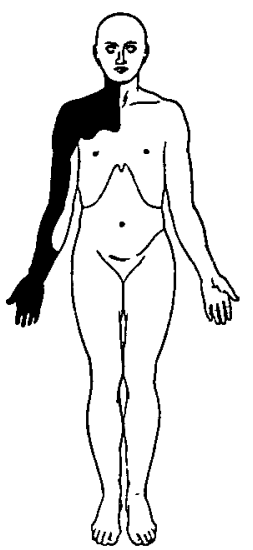

FIG. 75.
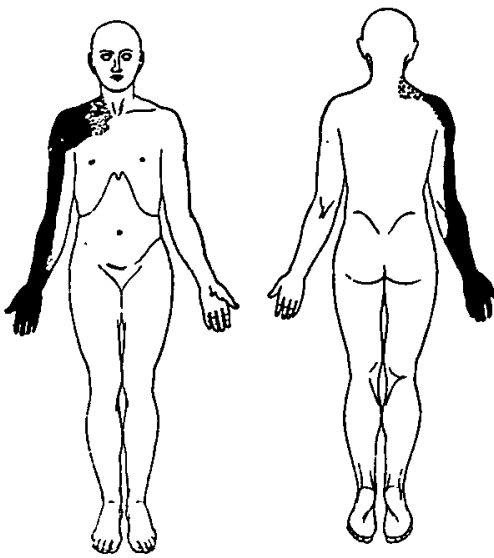

Fra. 76.

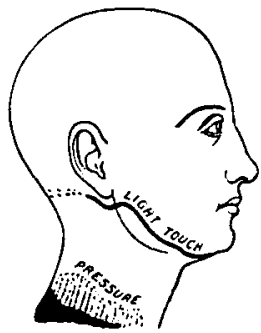

Fig. 77.

Fig. 75 shows the extent of the anæsthesia to cotton-wool in Case 15.

Fig. 76 shows the parts insensitive to deep touch in the same case.

Fig. 77 shows the relation of the borders at which different tactile stimuli no longer caused sensation. At the line marked " light touch" the patient ceased to appreciste cotton-wool and No. 8 of von Frey's hairs (830 mgm.). At the border of the dotted area pressure frequently failed to evole a response. Finally, over the black area no tactile stimulus, even the firmest pressure, was appreciated as a touch.

heat over the whole of the right upper extremity (Fig. 73). On the left side, the extent of the loss of sensation varied somewhat from day to day, and merged gradually into parts of normal sensibility. 
To cold the loss was less extensive and a small area remained sensitive on the inner aspect of the right arm (Fig. 74). Over the left hand and forearm, the distribution of the anæsthesia varied somewhat, corresponding to the evident advance of the lesion on this side of the nervous system.

(c) Tactile sensibility was abolished over the whole of the area shown on Fig. 76. The absolute loss of sensation to pressuretouch merged gradually on the neck into parts that were sensitive to this stimulus. But on the inner aspect of the forearm was a well-defined area of residual tactile sensibility.

When cotton-wool was used, the whole of the parts on Fig. 75 were found to be insensitive, including the right half of the neck to the line marked on Fig. 77.

The condition of the right half of the neck between these two lines was instructive; when tested with von Frey's hairs, it was found that even No. 8 (830 mgm.) could not be recognised, as soon as the border in the neighbourhood of the jaw was passed; and yet, over the left half of the neck he uniformly responded to No. 2 (100 mgm.) Tactile sensibility was sufficiently diminished at the line marked on the face to prevent a response to such stimuli as cotton-wool and von Frey's hairs. But No. 8 of these hairs is a pressure stimulus, and with peripheral lesions can be appreciated over parts of cutaneous insensibility. We concluded, therefore, that all forms of tactile sensibility were diminished at the line marked on Fig. 77. At this border, cotton-wool and von Frey's bairs ceased to cause a sensation. The anæsthesia gradually deepened until within the dotted area even a pressuretouch frequently failed to evoke a response. Finally, within the area marked black no tactile stimulus was appreciated.

(d) Acuasthesia.-He could tell the head from the point of a pin everywhere except over those parts of the right upper extremity insensitive to light touch. Over the area sensitive to pressure but not to light touch, he was unable to distinguish the head from the point. But, wherever he was sensitive to light touch within the analgesic area, his power of distinguishing the head from the point was perfectly preserved.

(e) Appreciation of differences in size could not be tested over the right upper extremity. But over the left hand and arm his answers were correct to rods of a diameter of $2 \mathrm{~cm} ., 0.75 \mathrm{~cm}$. and $0.2 \mathrm{~cm}$.

(f) The vibrations of a tuning fork could not be appreciated anywhere over the right upper extremity, but over the left arm and hand he everywhere answered correctly. 
(g) Sense of passive position and movement.-He could not recognise any movement of the fingers or thumb of the right hand, and was liable to drop anything he was holding unless his eyes were fixed upon it. He entirely failed to appreciate any movement of the fingers produced by electrical stimulation. Movements at the wrist were not recognised until they had exceeded $20^{\circ}$; at the right elbow, until they had exceeded $10^{\circ}$; but movements of the right shoulder-joint were readily recognised.

All movements of the left upper extremity and of the legs were readily appreciated.

(h) Compass-test.-This test could not be applied to the right upper extremity, on account of the loss of tactile sensibility. Elsewhere the records were normal.

The records obtained by testing the area on the right half of the neck insensitive to light touch but sensitive to pressure, show well the effect produced upon the compass-test by a general diminution of tactile sensibility, produced by an intra-medullary lesion.
R.
$3 \mathrm{~cm} . \frac{1 \mid 10 \mathrm{R} .}{2 \mid 4 \text { R. } 6 \mathrm{~W}}$.
L.
$1 \mid 10 \mathrm{R}$
$10 \mathrm{~cm} \cdot \frac{1 \mid 10 \mathrm{R} .}{2} \mid 5 \mathrm{~K} .1$ Doubtful. $4 \mathrm{~W}$.

(i) Tactile localisation.- Wherever he was able to recognise a touch, whether it were caused by pressure, cotton-wool, or von Frey's hairs, be invariably named the spot accurately. When allowed to grope for the spot touched he was never more than $3 \mathrm{~cm}$. away, and was remarkably quick and accurate, provided it lay over parts where tactile sensibility was preserved.

Cranial nerves.-The pupils were equal and reacted normally to light, to shade, and to accommodation. The eyes moved well in all directions, except that, when he looked to the right, a bilateral rotatory nystagmus appeared; the rotation occurred in a direction opposite to movement of the hands of a clock. No similar nystagmus was present when he looked to the left. No abnormal condition was found on ophthalmoscopic examination. All movements of the face and tongue were normally performed and the palate moved well.

Spine.-There was no lateral curve or other abnormality.

Sphincters.-For some time he had noticed that he could not retain his motions when they were loose and that he had some difficulty in starting micturition. 
Case 16.-Robert Arthur H.

In June, 1902, he wolke to find the left hand and arm numb; he noticed that over the numb parts heat and cold produced a sensation different from that over the normal parts.

Nine months later his throat became affected.

Painless swelling of the left shoulder-joint was present.

First seen March, 1904. His condition has advanced little since that date.

The motor affection consists of paralysis of the left vocal cord and the left half of palate.

Reflexes.-Left knee-jerk exaggerated, left ankle-clonus, left extensor plantar reflex.

Sensation.-Profound loss of sensibility to prick (Figs. 78 and 79), to thermal stimuli (Figs. 80 and 81), to all forms of tactile stimulation (Fig. 82).

Appreciation of passive position and movement lost in the left arm and left leg below the knee.

Tactile discrimination lost in the left leg; cannot be tested in the left arm on account of the loss of tactile sensibility.

Tactile localisation, (1) by naming, equally good in both legs, (2) by groping, profoundly changed in the left leg; normal in the right leg.

Robert Arthur H., aged 33, a general provision dealer, was first seen by us in March, 1904, and since then has been under observation from time to time.

He was apparently in perfect health until one morning in June, 1902, when he found on rising that his left hand and arm were numb. At the same time he noticed that heat and cold produced a different effect upon the sensation of this arm.

Nine months later, he began to suffer from choking sensations and his voice became affected. About the same time be found that the left leg troubled him in walking; it seemed stiff and awkward, although it " could feel perfectly." He then began to suffer from sensations of giddiness, and his eyesight became confused.

It was not until October 14, 1903, that the right hand was affected; it became red and swollen, and he suffered from a violent shivering attack in which he vomited. This was said to be due to a small poisoned wound for which the doctor bound up the hand with the fingers flexed into the palm. So he remained for a month, and, when the bandages were taken off, his fingers had stiffened permanently. 
About nine months later, the left shoulder-joint enlarged, but the swelling was accompanied by no pain.

As a boy, he had had an attack of typhoid fever, and on several occasions had suffered from "influenza," most severely in 1890.

He has been a life-long teetotaler.

No history could be obtained of venereal disease, nor according to his account is it likely that he could ever have been infected. He was married in 1899 and has one healthy child, born in 1905. His wife has never miscarried.

\section{Condition on Examination (November and December, 1906).}

In the following account we shall give the results of a series of examinations, extending over the last two months of 1906, alluding briefly when necessary to his condition on previous occasions.

$\mathrm{He}$ is a medium-sized, strongly-built man, of unusual intelligence. He answered well and did not fail with the control tests used.

Cranial nerves.-A remarkable nystagmus was present, not only with movement in all directions, but also when he looked in front with his eyes directed towards a distant point. When looking to the right or to the left, the nystagmus was purely horizontal and at no time was it truly rotatory. Otherwise, the eyes moved well, and there was no ocular paralysis. He had perfect muscular control of both eyelids. The face moved well both on emotional and voluntary excitation. The tongue was protruded in a straight line, but the right half seemed to be smaller than the left; this appeared to be due to a greater contraction of the muscles of the right half of the tongue, whilst the left half was flattened and flabby. The left half of the palate was paralysed, and on laryngoscopic examination, the left cord was seen to lie in the cadaveric position, completely motionless. The right vocal cord moved well, passing across the middle line to meet the left cord during phonation.

Motion.-There was no wasting of any muscle in the upper extremities, but the right hand was held in a half clenched position, and he was unable fully to extend the fingers in consequence of changes in the joints. The subcutaneous tissues were somewhat swollen, and the fixation of the fingers, combined with this swelling, accounted for the difference in the grasp of the two hands (right $8 \mathrm{kgm}$., left $17 \mathrm{kgm}$.).

But the grasp of the left hand was also defective, as shown by the low reading of the dynamometer; this was probably due, not to muscular weakness, but to the profound sensory changes in this hand. 
For the left upper extremity was profoundly ataxic; he could touch his nose clumsily when his eyes were open, but as soon as they were closed, the difficulty became greatly.increased. Even when allowed to look, he could scarcely bring the tips of the two forefingers together, and when his eyes were closed, this movement became impossible.

There was no ataxy of the right arm or hand.
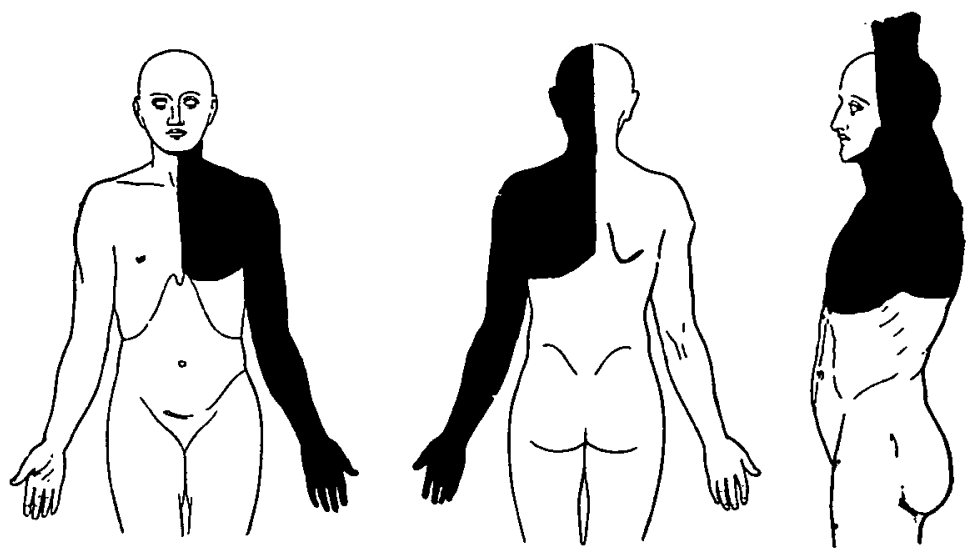

FiG. 78.

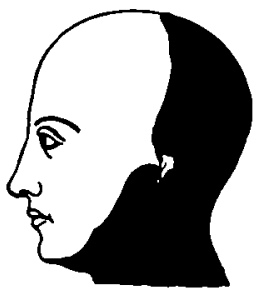

Fra. 79.

Figs. 78 and 79 show the loss of sensation to cutaneous painful stimuli in Case 16. The frontal border on the face (Fig. 79) was worked out by testing from sensitive towards insensitive parts. The caudal border shown on Fig. 78 was also worked out by determining the extent of residual sensibility to prick.

His gait was slightly unsteady, and on examination this was found to be due to the condition of the left leg. He could stand with his feet together, but when his eyes were closed, swayed and vltimately fell. In attempting to maintain his balance, the movements of the right foot were more extensive than those of the left; ultimately, he either fell towards the left, or thrust out his left leg laterally to act as a prop. Asked to stand on the 
right leg only, he finally succeeded; but at first he had difficulty in maintaining his balance, owing to the absence of compensatory movements in the left leg, which was lifted. When he attempted to stand on the left leg only, he at once fell. Under such con. ditions, there was a striking difference between the violent balancing movements of the right foot and the complete quiescence of the left foot.

The left leg was slightly spastic, but was not paretic; nor were the muscles wasted.

All the muscles of the upper and lower extremities reacted perfectly to the interrupted and to the constant current.

Reflexes.-The left knee-jerk was greatly exaggerated, and ankle-clonus was obtained; the left plantar reflex gave an extensor response. On the right side the knee-jerk was obtained, but there was no ankle-clonus, and the plantar reflex was of the flexor type.

Sensation.-The loss of sensation was slightly less extensive in March, 1904, than in 1906. But since the changes which have occurred during the last two and a half years consist solely of a slight increase in the area affected, and not in the disturbance of any further sensory quality, we shall contine ourselves mainly to the present condition.

(a) Sensibility to painful stimuli.-He was insensitive to the prick of a pin over the whole of the left upper extremity, and over the left half of the neck and scalp (Fig. 78). The frontal border of the analgesia extended on to the face, including the left ear and part of the left cheek; the scalp was insensitive to a point a little in front of the vertical line joining the two ears (Fig. 79).

The lower border of the analgesic area on the trunk was remarkably well defined; it extended round the trunk, crossing the sixth, seventh, and eighth ribs in the axilla to end at the level of the sixth vertebral spine. When he was tested from parts of normal sensibility towards those that were analgesic, sensation to the prick of a pin or to a painful interrupted current ceased at the border on Fig. 78; from this, the border of returning sensibility scarcely differed by one centimetre, when we tested from insensitive towards sensitive parts.

Sensibility to a pin prick and to the painful interrupted current was perfect elsewhere over the body, except that it was somewhat diminished over the right forearm.

With the algometer, it was found that sensibility to the pain of excessive pressure was lost over the area insensitive to cutaneous painful stimuli. Over the right arm, the algometer 
gave somewhat higher readings where cutaneous sensibility to pain was diminished.

$\mathbf{R}$

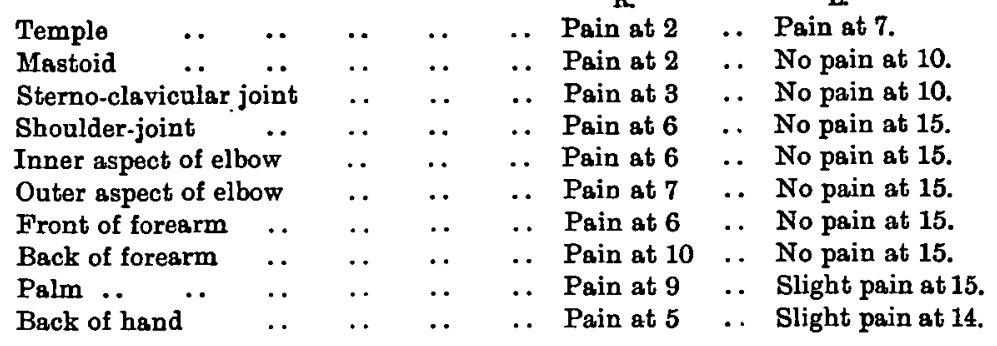

Outer aspect of anterior superior spine of Pain at $3 \quad \ldots \quad$ Pain at 3.

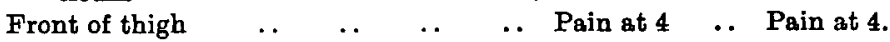

$\begin{array}{lllllll}\text { Inner aspect of knee } & \ldots & \ldots & \ldots & \text { Pain at } 4 & \text {. } & \text { Pain at } 4 .\end{array}$

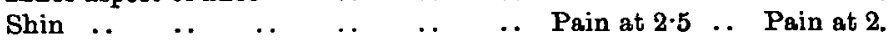

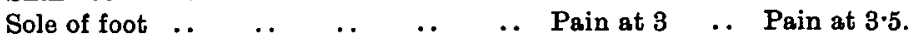

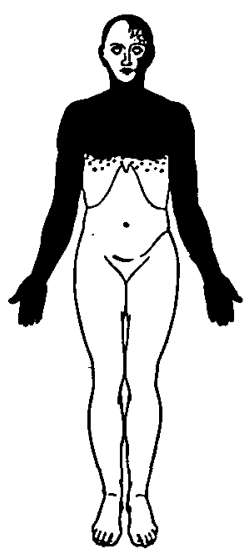

Fig. 80.

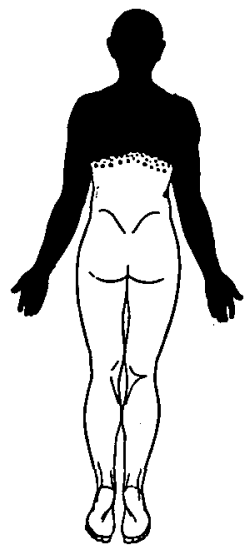

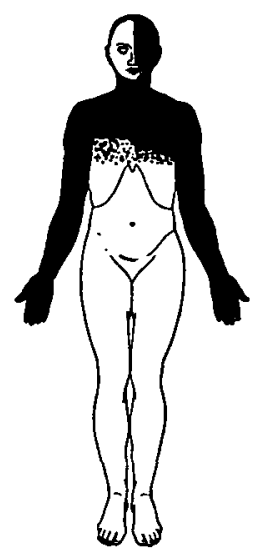

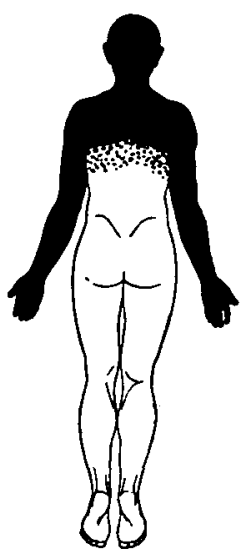

Fig. 81.

Fig. 80 to show the extent of the loss of sensation to cold, and fig. 81 to show the loss to heat in Case 16.

(b) Thermal sensibility.-The area insensitive to all degrees of cold between $0^{\circ}$ and $25^{\circ} \mathrm{C}$. is shown on Fig. 80 . The parts insensitive to all degrees of heat are marked on Fig. 81 . These two figures almost correspond with one another except on the face. But, in 1904, the loss to cold considerably exceeded the loss to heat; the area insensitive to cold nearly corresponded with that on Fig. 80, but the extent of the anæsthesia to heat 
more nearly agreed with the area that is now insensitive to painful stimuli (Fig. 78). Thus, at this time the whole of the right arm was sensitive to heat, but insensitive to cold.

(c) Tactile sensibility. - Stimulation with cotton wool was not appreciated over the whole of the left upper extremity, and the left half of the chest above the level of the nipple (Fig. 82). Elsewhere over the body and limbs, this stimulus was perfectly appreciated.

Firm pressure was not appreciated over the same area (Fig. 82) as that insensitive to light touch. This complete abolition of tactile sensibility over the left upper extremity was present when he was first examined in March, 1904.

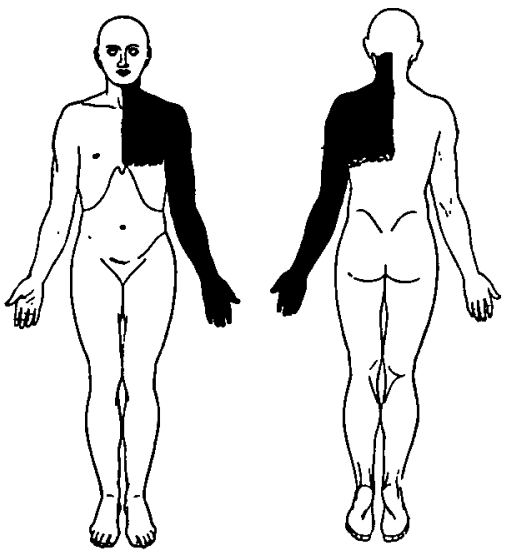

Fig. 82.

To show the loss of sensibility to stimulation with cotton-wool, with von Frey's hairs and with pressure-touch in Case 16.

(d) Passive position and movement.-He stated that he was able to appreciate the movements of the fingers of his left hand; but when they were moved passively he never recognised the movement, until the finger had travelled through an angle of over $60^{\circ}$. He was entirely unable to say into what position the fingers of the left hand had been placed. When the left thumb was tested, he answered as follows :-

$$
\begin{array}{l|l}
\text { Flexion } & 1 \text { R. } 9 \mathrm{~W} . \\
\hline \text { Extension } & 2 \mathrm{R} .8 \mathrm{~W} .
\end{array}
$$

When allowed to look at the true position of his thumb, he volunteered that he thought it was pointing in a different direction. 
At the left wrist-joint, he appreciated flexion and extension when movement had been made through an angle of $40^{\circ}$. He was, bowever, always correct in his appreciation of the position into which the hand had been placed by passive movements at the wrist, although his answers were somewhat slow.

Passive position and movement at the left elbow and shoulder were quickly and accurately appreciated; he recognised passive movement through $5^{\circ}$ only.

In the whole of the right upper extremity, passive position and movement were accurately appreciated, and the difference between the condition of the right (normal) and left (affected) hands was most evident.

But it was in the lower extremities, where all other forms of sensibility were perfect, that the disturbed appreciation of passive position and movement was most striking. When his left great toe was moved, his answers were hesitating and very uncertain, but he quickly and accurately recognised all movements of the right great toe.

$$
\begin{aligned}
& \text { Right. Left. } \\
& \text { Great Toe } \frac{\text { Extension | 10 R. }}{\text { Flexion | 10 R. }} \quad \frac{\text { Extension |4 R. } 6 \mathrm{~W}}{\text { Flexion |9R.1W. }}
\end{aligned}
$$

He could not recognise movements of the left ankle, until it had been moved through more than $20^{\circ}$, whilst on the right side he answered correctly with movements of less than $5^{\circ}$.

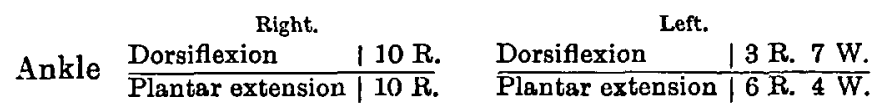

At the knees and hips his answers were equally accurate on the two sides.

At a later date the following similar results were obtained :-

Right.

Left.

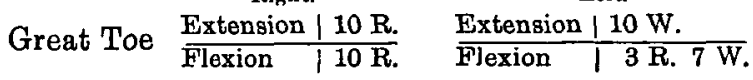

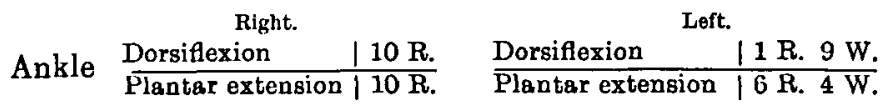

The same facts were illustrated when the movements of the foot were produced by electrical stimulation of the muscles. If the right foot was dorsiflexed by the application of an interrupted current to the anterior tibial group of muscles, or plantar extended by stimulating the calf, he answered quickly, repeating the move- 
ment correctly in the same leg. The same result followed the outward movement produced by stimulation of the peronei.

But, when similar stimulation was applied to the muscles of the left leg, he was completely puzzled, and answered "the leg jumps," whatever movement resulted.

(e) The compass-test.-Owing to the loss of tactile sensibility in the left upper extremity it was impossible to compare the behaviour of the two hands towards the compass-test.

But over the lower extremities, where tactile, painful and thermal sensibility were perfect even to the finest tests, a remarkable difference was discovered in the behaviour of the two limbs. For over the right sole he made one mistake only when the points were separated for a distance of $2.5 \mathrm{~cm}$., and applied transversely; over the left sole he gave eight false answers when the points were $6 \mathrm{~cm}$. apart. Over the outer aspect of the left leg he gave perfect answers at $8 \mathrm{~cm}$, but over the right leg $15 \mathrm{~cm}$. was well below the threshold.

Sole :

\begin{tabular}{|c|c|c|}
\hline $\begin{array}{l}\text { Compasses applied } \\
\text { transversely }\end{array}$ & 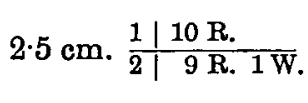 & $6 \mathrm{~cm} . \frac{1 \mid 10 \mathrm{R} .}{2 \mid 2 \mathrm{R} .8 \mathrm{~W}}$ \\
\hline Compasses applied & $4 \mathrm{~cm} . \frac{1 \mid 10 \mathrm{R}}{2 \mid 10 \mathrm{R} .}$ & $4 \mathrm{~cm} \cdot \frac{1 \mid 10 \mathrm{R}}{2 \mid 10 \mathrm{w}}$ \\
\hline longitudinally & & $8 \mathrm{~cm} \cdot \frac{1 \mid 10 \mathrm{R} .}{2 \mid 4 \mathrm{R} .6 \mathrm{~W}}$ \\
\hline
\end{tabular}

Outer aspect of leg $\quad . \quad 8 \mathrm{~cm} \cdot \frac{1 \mid 10 \mathrm{R} .}{2 \mid 10 \mathrm{R} .} \quad 15 \mathrm{~cm} \cdot \frac{1 \mid 6 \mathrm{R} .4 \mathrm{~W}}{2 \mid 5 \mathrm{R} .5 \mathrm{~W}}$.

(f) Tactile localisation.-(1) Tested by the power of naming the part touched.

A number of points were chosen, such as the ball of the great toe, the centre of the sole of the foot, the heel, the "outer ankle" (external malleolus), "the inner part of the leg over the calf," "outer side, and just below the knee," the knee-cap, the " centre of the calf," the " middle of the front of the thigh." All these points when touched were named quickly and accu. rately; no difference could be discovered between the two lower extremities. Nor did he seem to be slower in naming a point touched on the lower extremities than when he was touched on the right hand or arm.

Then his eyes were closed and the legs were moved passively into a fresh position. This movement in the right leg was fully appreciated, but he was unable to tell into what position his left 
foot and leg had been placed; yet, in spite of this difference, he named the spots touched on the two legs in their new position with equal accuracy.

(2) Tested by Spearman's modification of the groping method.

$A$ hole was cut in the centre of a piece of thin cardboard, on to which was fastened a sheet of white paper. The patient sat propped up in bed, with his legs stretched out in front of him. He was given a long soft-pointed pencil, and his eyes were closed. The cardboard was then held over his foot about $1 \mathrm{~cm}$. from the skin, so that the hole lay over the centre of the dorsal surface. Through this hole he was touched, and he then attempted to touch the spot with the pencil used as a pointer. Now, since the cardboard intervened between his foot and the pointer, except at the small hole, his groping efforts were marked on the white paper, and the distance of the final mark from the centre of the hole gave the measure of his accuracy. A series of experiments were made with the centre hole over similar points on the dorsun of the right and left foot, on the outer aspect of the legs, and on the front of the thighs. Six tactile stimuli were given at each spot, and the numbers given below represent the average deviation at each point.

\begin{tabular}{|c|c|c|c|c|c|}
\hline & & & R. (normal). & & L. (affected) \\
\hline Dorsum of the foot.. & .. & .. & $5.7 \mathrm{~cm}$ & .. & $7 \mathrm{~cm}$ \\
\hline Outer aspect of leg & $\cdots$ & $\cdots$ & $4 \cdot 3 \mathrm{~cm}$ & . & $6.8 \mathrm{~cm}$ \\
\hline Front of thigh & . & .. & $2.5 \mathrm{~cm}$ & $\cdots$ & $4.3 \mathrm{~cm}$ \\
\hline
\end{tabular}

The deviation was uniformly a little greater on the left than on the right leg, but the difference was within the limits of error of observation.

Another set of experiments was made as follows: The legs were allowed to lie stretched out in front of him and his eyes were closed. A series of observations were made exactly in the same way as those quoted above. Then his legs were moved passively, his eyes remaining constantly closed, and another set of observations made by touching the same spot on the dorsum of his foot. The results can be tabulated as follows :-

$\begin{array}{llllcrcr} & & & & \text { R. (normai). } & & \text { L. (affected). } \\ \text { Before movement } & \ldots & \ldots & \ldots & 9.7 \mathrm{~cm} . & \ldots & 9 \mathrm{~cm} . \\ \text { After movement } & \ldots & \ldots & \ldots & 9.7 \mathrm{~cm} . & \ldots & 13 \mathrm{~cm} .\end{array}$

It will be seen that the deviation on the sound side remained the same, although the leg had been displaced passively; but on the affected foot the deviation increased $4 \mathrm{~cm}$. after the foot had been moved.

(g) Acucsthesia.-Under normal circumstances be never made 
a mistake between stimulation with the head and the point of a pin over the right arm and hand, right half of the trunk, and both lower extremities. The left upper extremity was insensitive to touch and pain.

But the following observation will serve as an instance of the erroneous answers which may be given, if the patient is in any way uncomfortable. On one occasion at the end of a morning's examination this test was applied to the right arm. He gave curiously false answers as follows :

$$
\begin{aligned}
& \text { Right palm } \frac{\text { Head | } 2 \text { R. } 8 \mathrm{~W} .}{\text { Point | 10 R. }} \\
& \text { Right forearm } \\
& \frac{\text { Head | 4 R. } 6 \mathrm{~W}}{\text { Point | } 10 \mathrm{R} .}
\end{aligned}
$$

He then said he wished to pass urine, which he did copionsly. From that time he gave perfect answers every where over the right arm, just as on previous occasions.

(h) Appreciation of difference in size.- This was tested with three metal cylinders, $2 \mathrm{~cm}$., $0.75 \mathrm{~cm}$., and $0.4 \mathrm{~cm}$. in diameter. Except over the left upper extremity his answers were correct in every instance.

\section{Case 17.-Frank L.}

On. August 3, 1904, he was injured by the fall of a skip which struck him in the back.

On August 9, the spine was opened in the region of the first, second and third lumbar vertebre. No abnormality was discovered, showing that the condition was not due to injury of the cauda equina.

He gradually recovered and, when seen by us in May, 1906, showed some spasticity of the left leg, associated with analgesia and thermo-anasthesia of the right leg (Fig. 83).

Over this area, he was sensitive to the tactile aspect of pressure, but excessive pressure produced no pain.

All other forms of sensibility were perfectly preserved.

Frank L., aged 25, was admitted to the London Hospital on August 3,1904 , in consequence of an accident when working on the Rotherhithe tunnel. A skip of concrete fell, striking him in the lower thoracic and upper lumbar regions. He did not lose consciousness, but when pulled from under the skip, his legs dropped as if they were broken.

When admitted to the London Hospital, a bæmatoma was 
present over the second, third and fourth lumbar spines, and he was much bruised about the body. The knee-jerks and plantar reflexes were absent, and both legs were completely paralysed.

He did not seem to be improving, and on August 9, 1904, Mr. Rigby operated upon the spine. He removed the laminæ of the first, second and third lumbar vertebræ. The dura mater was opened, and the cauda equina was exposed. No signs of rupture or bruising could be discovered anywhere. The wound was sewn up again and healed by first intention,

Power considerably improved in both legs, and he gained control to a great extent over micturition. When first examined by us, he had reached the following condition.

\section{Condition in May, 1906.}

He was a largely-built, intelligent man.

Motion.-When walking, he dragged the left leg like a man with hemiplegia, raising his pelvis with each step so that the left foot should clear the ground. He could stand on either leg, but could not support bimself on the left leg only for more than a few seconds.

This leg was stiff, especially below the knee, and he complained that he could not move it as quickly and easily as the right. All movements at the left hip and knee were strongly performed, but at the left ankle he moved slowly, although with considerable force. He could dorsiflex the foot, and plantar extension was good.

All movements of the right leg were well performed and it was not stiff or spastic.

Reflexes.-Both knee-jerks were exaggerated, the left more so than the right. Ankle clonus was obtained on both sides and both plantar reflexes gave an extensor response.

Spine.-The scar of a well-healed wound extended from the eleventh thoracic to the fourth lumbar spine.

A radiograph of the dorsal region of the spinal column showed no obvious signs of fracture or other bony change.

Sphincters.-He could pass bis water normally; but if he desired to micturate he was forced to do so at once, or the urine ran from him.

Defæcation was not affected in May, 1906.

Sensation. (a) Tactile sensibility.- $\mathrm{He}$ was sensitive everywhere to cotton-wool, and no difference in this respect could be discovered between the two legs. He recognised increasing pressure equally over both lower limbs. 
(b) Sensibility to pain.-He was insensitive to all forms of cutaneous painful stimulation over the area shown in Fig. 83.

Deep pressure caused no pain over the sole of the foot, nor over both aspects of the right leg. But, over the front of the thigh, above the limits of the analgesia, pain was produced by a pressure of 5 kilograms on both sides.

R.

I.

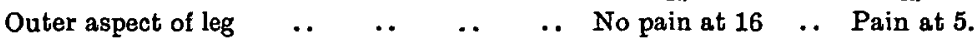
Inner aspect of leg over tibia.. $\quad \ldots \quad \ldots$ Nó pain at $10 \quad \ldots$ Pain at 4.

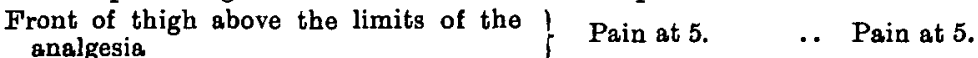
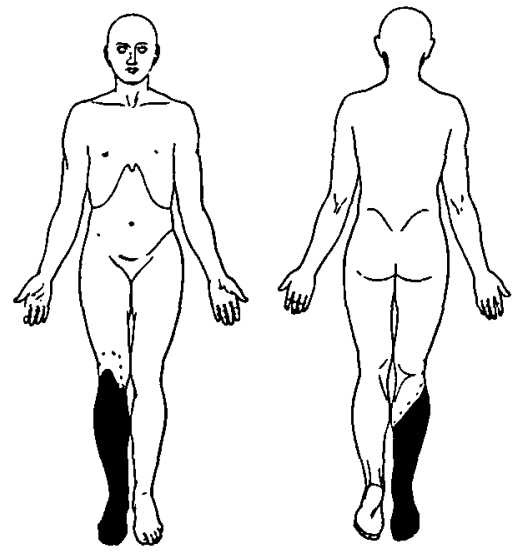

Frg. 83.

To show the loss of sensation in Case 17.

The dark area represents the parts insensitive to cutaneous painful stimuli and also to the pain of excessive pressure. It also corresponds to the extent of the thermo-anæsthesia.

(c) Thermal sensibility was lost for all degrees of heat and cold over the area marked in Fig. 83. The borders of this loss of sensation were ill-defined.

(d) The vibrations of a tuning fork (128) were appreciated with equal ease over both legs.

(e) The sense of passive position and movement was preserved everywhere.

(f) The compass-test.-The threshold over the outer aspect of the shins lay between 4 and $6 \mathrm{~cm}$. when the compasses were applied longitudinally. We were unable to discover any difference between the two legs in their sensibility to this test.

(g) Tactile localisation was everywhere good. 


\section{Description of Plates.}

\section{Plate I.}

Photographs of the spinal cord from Case 9 stained by the Weigert-Pal method. It will be seen that the whole of the left half was destroyed by a growth which occupied the centre of the cord. The white matter of the right half of the cord was distorted, but took the stain well.

Prate II.

Photographs of the spinal cord from Case 10 to show the focal lesion. Fig. 1 shows the sixth thoracic free from hæmorrbage or growth.

\section{Plate III.}

Photographs of the spinal cord from Case 10 stained by Busch's modification of the Marchi method. Fig. 1 shows both the extreme frontal extension of the focal lesion and the ascending degeneration. In the remaining figures all the ascending tracts are seen to be degenerated on both sides. (Cf. p. 697.) 


\section{References.}

[1] Bayliss. "On the Origin from the Spinal Cord of the Vasodilator Fibres of the Hind Limb, and on the Nature of these Fibres." Jour. nal of Physiology, 1900-1901, vol. xxvi., p. 173.

[2] Brown-Séquard. "Exposé Critique des idées de M. Chauveau, \&c." Journal de la Physiologie, 1858, Tom. 1.

[3] Brown-SÉquard. "Expériences nouvelles sur la transmission des impressions sensitives dans la Moelle épinière." Journal de la Physiologie, 1859, Tom. 2, p. 65.

[4] Brown-SÉquard. "Lectures on the Physiology and Pathology of the Central Nervous System." Philadelphia, 1860.

[5] BROWN-SÉquARD. "Recherches sur la transmission des impressions dans la Moelle épinière." Journal de la Physiologie, 1863, Tom. 6, pp. 124, $232,581$.

[6] Brown.Séquard. “Nouvelles Recherches sur le trajet des diverses espèces de conducteurs d'impressions sensitives dans la Moelle épinière." Archives de Physiologie, 1868, Tom. 1, pp. 610, 716; 1869, Tom. 2, pp. 236, 693.

[7] Brown-Séquard. "Lectures on the Physiology and Pathology of the Nervous System." Lancet, 1868, vol. ii., pp. 593, 659, 755, 821.

[8] Förster. "Untersuchungen über das Localisationsvermögen bei Sensibilitätsstörungen." Monatsschrift f. Psychiatrie u. Neurologie, 1901, Bd. ix., S. 31 .

[9] Fönster. "Die Physiologie u. Pathologie der Co-ordination." Jena, 1902.

[10] Frey, Max von. "Untersuchungen über die Sinnesfunctionen der menschlichen Haut." Abhandlungen a. mathematisch-physicalischen Classe d. königl. sächsischen Gesellschaft d. Wissenschaften, 1896, Bd. xxiii, No. iii., S. 175.

[11] Hrad. " Pain in Visceral Disease." Part 1, Brain, 1893, p. 1; Part 2, Brain, 1894, p. 339 ; Part 3, Brain, 1896, p. 153.

[12] Head and Campbell. "The Pathology of Herpes Zoster and its bearing on Sensory Localisation." BrAIN, 1900, p. 353.

[13] Head AND Sherren. "Consequences of Injury to the Peripheral Nerves in Man." Brain, 1905, vol. xxviii., p. 116.

[14] Henri, Victor. "Ueber die Raumwahrnehmungen des Tastsinnes." Berlin, 1898.

[15] Kоснев. "Die Verletzungen d. Wirbelsäule zugleich als Beitrag zur Physiologie des menschlichen Rückenmarks." Mittheilungen aus $d$. Grenzgeb. d. Medicin. u. Chir., 1896, Bd. 1, Hft. 4.

[16] LaEhr, Max. "Ueber Storüngen d. Schmerz und Temperatur-empfin. dung in Folge von Erkrankungen des Rückenmarks." Archiv. für Psychiatrie, 1896, Bd. xxviii., S. 773.

[17] Lewandowskr. "Die Functionen des zentralen Nervensystems." Jena, 1907.

[18] Mac Dougall. " Reports of the Cambridge Anthropological Expedition to Torres Straits." Cambridge, 1903, vol. ii., part ii., p. 189.

[19] Motr. "Results of Hemisection of the Spinal Cord in Mlonkeys." Phil. Trans. Roy. Soc., 1892, vol, clxxxiii., B., p. 1.

[20] Oppenheim. "Lehrbuch dar Nervenkrankheiten." Vierte Auflage. Berlin, 1905. 
[21] Petrén, Karc. "Ein Beitrag zur Frage vom Verlaufe d. Bahnen d. Hautsinne im Rückenmarke." 1902, Skandinav. Archiv. für Physiologie, Bd. xiii., S. 9.

[22] PrLtz. "Ein Beitrag zum Studium d. Dissociation d. Temperatur- und Schmerzempfindung bei Verletzungen und Erkrankungen des Rückenmarks." Archiv. fiur Psychiatrie, 1906, Bd. 41, Hft. 3, p. 951.

[23] Rivers, Sherren and Head. "The Afferent Nervous System from a New Aspect." BraIN, 1905, vol. xxviii., p. 99.

[24] RothmanN, Max. "Ueber die Leitung d. Sensibilität im Rückenmark." Berlin. Klin. Wochenschrift, 1906, No. 2, Jan. 8; No. 3, Jan. 15.

[25] Schlesinger. “Die Syringomyelie." Leipzig, 1902.

[26] Schüster. "Untersuchungen ü. d. Sensibilitätsleitung im Rückenmark d. Hundes." Monatsschr.f. Psychiatrie $u$. Neurologie, 1906, Bd. 20, Hft. 2, S. 97.

[27] SelfFen. "Das spinale Sensibilitätsschema zur Segment-diagnose der Rückenmarks-krankheiten." Archiv, für Psychiatrie, 1901, Bd. 34, Hf. 2 , S. 648 .

[28] Sherren. "The Distribution and Recovery of Peripheral Nerves." The Erasmus Wilson Lectures at the Royal College of Surgeons of England. Lancet, March 17, 24, 31, 1906.

[29] Sherren. "A Case of Secondary Suture of the Great Sciatic Nerve." Brit. Med. Journ., Feb. 16, 1907.

130] Sherrington. "Experiments in Examination of the Peripheral Distribution of the Fibres of the Posterior Roots of some Spinal Nerves. Part 1. Phil. Trans. Roy. Soc., 1893, vol.clxxxiv., B.p. 641. Part 2. Phil. Trans. Roy. Soc., 1898, vol. cxc. B., p. 45.

[31] Spearman, C. "Analysis of 'Localisation,' illustrated by a BrownSéquard case." Brit. Journal of Psychology, 1905, vol. 1., part 3, p. 286.

[32] Spearman, C. "Fortschritte auf d. Gebiete d. Psychophysik d. räum. lichen Vorstellungen." Archiv. d. gesamten Psychologie, 1906, Bd. viii., Hft. 1 u. 2, Literaturbericht, S. 1.

[33] Stark, Allen. "Local Anæsthesia as a Guide in the Diagnosis of Lesions of the Lower Spinal Cord." American Journal of the Med. Sci., 1892, vol. civ., p. 15.

[34] Starr, Alcen. "Local Anæasthesia as a Guide to the Diagnosis of Lesions of the Upper Portion of the Spinal Cord." Brain, 1894, vol. xvii., p. 481.

[35] Thoвbunn. "A Contribution to the Surgery of the Spinal Cord." London, 1889.

[36] Thonburn. "The Sensory Distribution of Spinal Nerves." Brain, 1893 , vol. xvi., p. 355.

[37] Torner. "On Hemisection of the Spinal Cord." Brain, 1891, vol. xiv., p. 496.

[38] Volkarans. "Nervenphysiologie." Wagner's Handwörterbuch d. Phys iologie, Bd. 2, S. 571 .

[39] Weber, E. H. "Der Tastsinn und das Gemeingefühl." Wagner's Handwörterbuch der Physiologie, 1846, Bd. iii., Abth. 2, S. 481.

[40] Wrahman, RaLf. "Die Rückenmarksnerven und ihre Segmentbezïge.' Berlin, 1900. 